\title{
Health consequences of group living in wild Verreaux's sifakas (Propithecus verreauxi)
}

\author{
Dissertation \\ for the award of the degree \\ "Doctor rerum naturalium" (Dr. rer. nat.) \\ of the Georg-August-Universität Göttingen
}

within the doctoral program Behaviour and Cognition (BeCog)

of the Georg-August University School of Science (GAUSS)

submitted by

Katja Rudolph

from Leipzig, Germany

Göttingen, 2020 


\section{Thesis committee}

Dr. Claudia Fichtel

Behavioural Ecology and Sociobiology Unit, German Primate Centre, Leibniz Institute for Primate Research, Göttingen

Prof. Dr. Julia Ostner

Behavioural Ecology, Georg-August University, Göttingen \& Primate Social Evolution, German Primate Centre, Leibniz Institute for Primate Research, Göttingen

Prof. Dr. Rolf Daniel

Department of Genomic and Applied Microbiology, Georg-August University, Göttingen

\section{Members of the Examination Board}

Reviewer: $\quad$ Dr. Claudia Fichtel

Second reviewer: $\quad$ Prof. Dr. Julia Ostner

\section{Further members of the Examination Board}

Prof. Dr. Peter M. Kappeler

Behavioural Ecology and Sociobiology Unit, German Primate Centre, Leibniz Institute for Primate Research, Göttingen

Prof. Dr. Rolf Daniel

Department of Genomic and Applied Microbiology, Georg-August University, Göttingen

Prof. Dr. Lars Penke

Biological Personality Psychology Georg-August University, Göttingen

Dr. Dietmar Zinner

Cognitive Ethology Laboratory, German Primate Centre, Leibniz Institute for Primate Research, Göttingen 


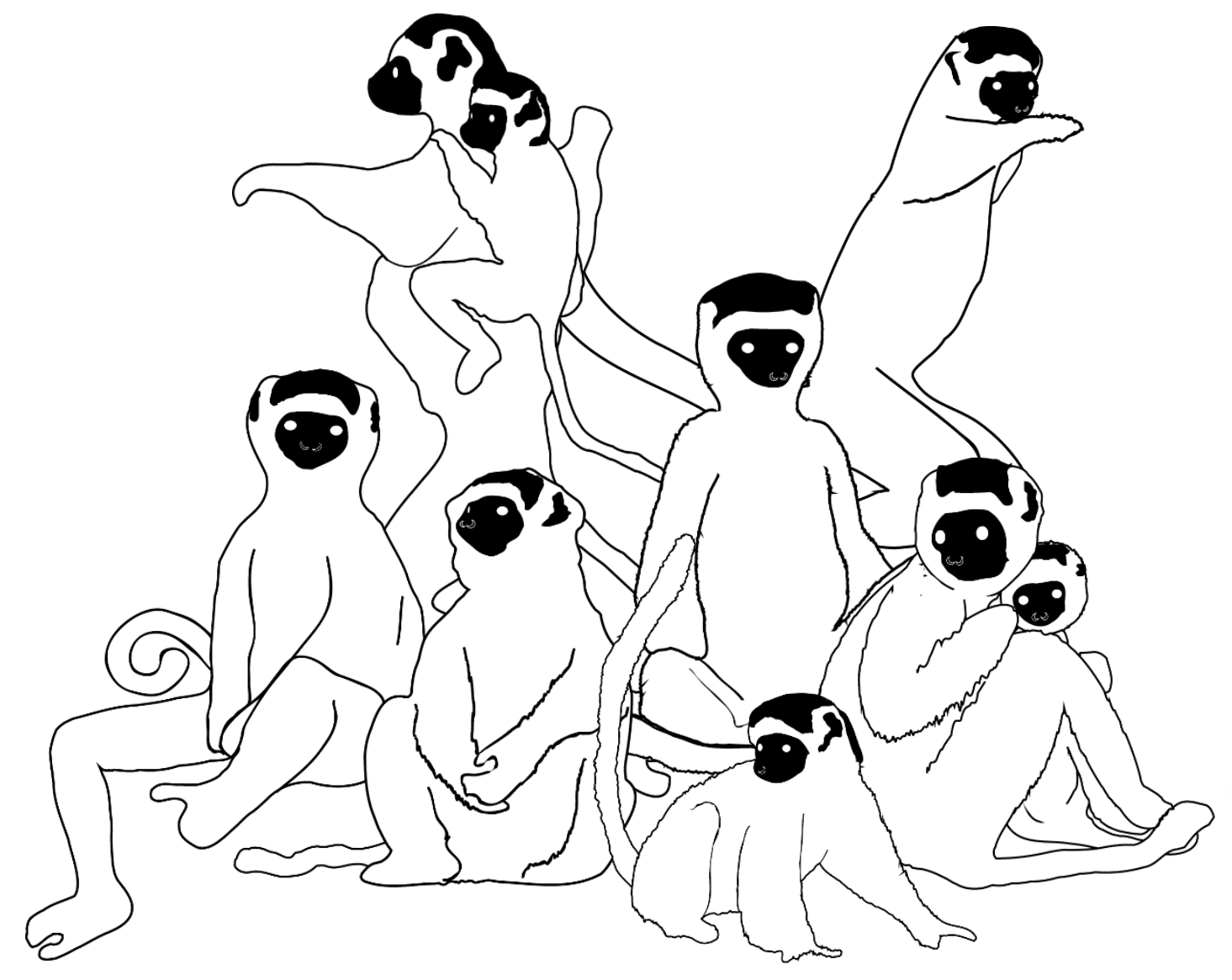




\section{Table of Contents}

Summary

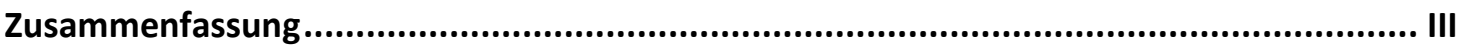

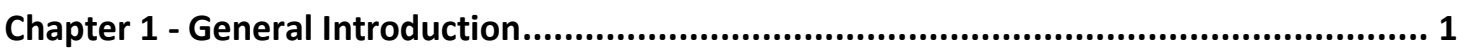

1.1 Towards Understanding the Evolution of Sociality ........................................

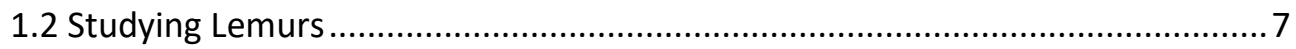

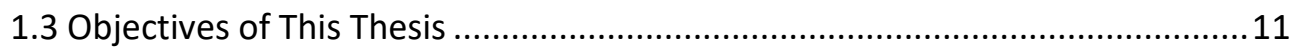

Chapter 2 - One Size Fits All? Relationships Among Group Size, Health, and Ecology Indicate a Lack of an Optimal Group Size

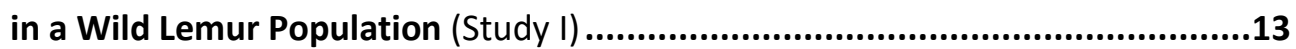

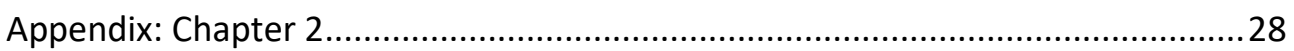

Chapter 3 - Dynamics and Determinants of Glucocorticoid Metabolite Concentrations in Wild Verreaux's Sifakas (Study II) ............................................................53

Chapter 4 - Exploring Causes for Gut Microbiome Variation among Groups of Wild Verreaux's sifakas (Study III) ................................................................55

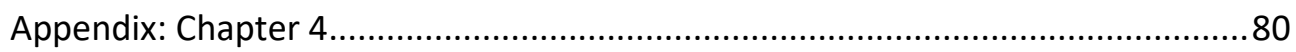

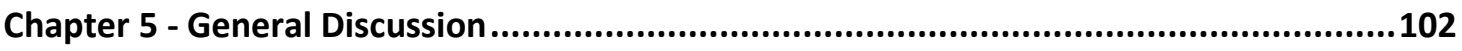

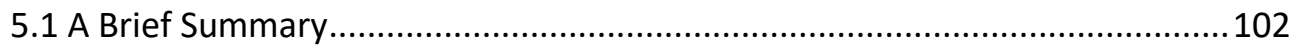

5.2 Limited Health-Related Consequences of Sociality in Verreaux's Sifakas .......103

5.3 Measuring Health in the Wild ....................................................................110

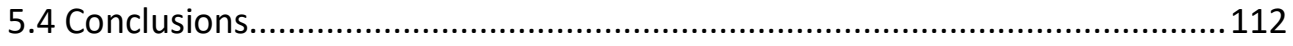

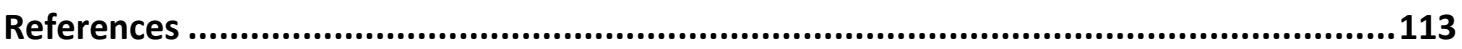

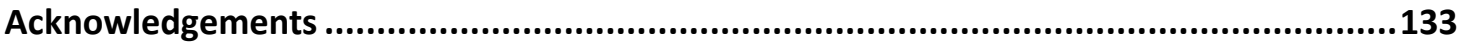

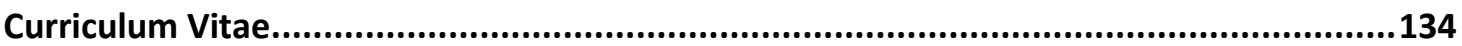

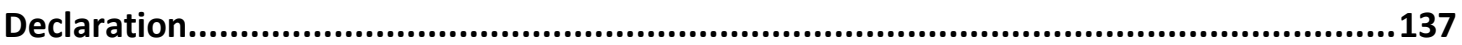




\section{Summary}

The evolution of sociality exposed individuals to several new health-related costs and benefits, which fundamentally affect their survival and reproductive success. Group living provides better access to food and mates and reduces risks of predation, while it also increases competition over shared resources and facilitates the transmission of pathogens. Whereas the general factors favouring the evolution of group living have been well established, little is known about the underlying mechanisms that link aspects of sociality with health. One reason for this limited understanding is the low number of longitudinal studies that have systematically examined this relationship in wild populations.

Here, I add needed data to this field of research by studying links among sociality and indicators of health in 42 individuals of seven neighbouring groups of a wild lemur population - Verreaux's sifakas (Propithecus verreauxi). These diurnal primates live in multi-male multi-female groups, have a mainly folivorous diet and inhabit the dry forests of southern and south-western Madagascar, where they are exposed to pronounced seasonality. I examined various aspects of Verreaux's sifakas' social life, i.e. group size, group membership, rank, affiliative and agonistic interactions, and their potential associations with activity and ranging patterns, parasite infections, measures of faecal glucocorticoid metabolites (fGCMs) and gut microbial communities collected over a study period of two consecutive years.

No individual showed signs of sickness and I found only few implications for health-related consequences of sociality in this species. More precisely, group size and social interactions had no impact on behavioural or physiological health-parameters. Between-group variation in activity and ranging patterns likely reflected adaptations to differences in microhabitat features and could be compensated without inflicting changes in fGCMs or parasite infections, i.e. health-related costs. Proximity to group members facilitated the transmission of microorganisms, indicating that gregariousness in this species may indeed come with the cost of parasite transmission, whereas the exchange of beneficial gut microbiota might improve individual health. The results of my thesis imply that health-related consequences of different aspects of group living in Verreaux's sifakas are limited to the effects caused by social proximity but not social interactions.

In conclusion, the magnitude of health consequences of sociality depends on species-specific aspects of their social systems. For example, highly competitive societies with higher rates of agonistic and affiliative interactions among individuals may increase variation in energetic and social stress and facilitate opportunities for transmission of microorganisms. On the contrary, in species with less competitive regimes and low interaction rates, like Verreaux's sifakas, health-consequences of sociality might be attenuated. More comprehensive wildlife studies conducted on species with different social systems and within an ecologically meaningful context are required to improve our understanding of the complex and interrelated factors that contribute to the sociality-health link. 


\section{Zusammenfassung}

Die Evolution des Soziallebens brachte mehrere neue vor- und nachteilige Konsequenzen für die Gesundheit von Individuen mit sich, welche wesentlichen Einfluss auf deren Überleben und Reproduktionserfolg haben. Das Leben in Gruppen ermöglicht besseren Zugang zu Nahrung und Fortpflanzungspartnern und vermindert das Prädationsrisiko, erhöht aber den Wettstreit um geteilte Ressourcen und vereinfacht die Übertragung von Pathogenen. Während die Faktoren, welche die Evolution des Gruppenlebens begünstigt haben, fest etabliert sind, bleibt das Wissen über die Mechanismen, die dem Zusammenhang von Sozialität und Gesundheit zu Grunde liegen, gering. Ein Grund für dieses begrenzte Verständnis liegt in der bisher geringen Zahl an Langzeitstudien, die den Zusammenhang von Sozialität und Gesundheit systematisch in Wildpopulationen untersucht haben.

Die Erkenntnisse der hier durchgeführten Studie tragen zur Erweiterung des Forschungsfeldes bei, indem Zusammenhänge zwischen Sozialität und Gesundheitsindikatoren bei 42 Tieren aus sieben benachbarten Gruppen einer wildlebenden Population von Lemuren untersucht werden - Verreaux's Larvensifakas (Propithecus verreauxi). Diese tagaktiven Primaten leben in Gruppen bestehend aus mehreren Männchen und Weibchen, ernähren sich von vorwiegend von Blättern und bewohnen die Trockenwälder im Süden und Südwesten Madagaskars, wo sie jährlich einem ausgeprägten Wechsel zwischen Regen- und Trockenzeit ausgesetzt sind. Über einen Zeitraum von zwei Jahren, wurden verschiedene Aspekte des Soziallebens der Tiere, d. h. Gruppengröße und -zugehörigkeit, Rang, freundliche und agonistische Interaktionen, und potenzielle Assoziationen mit Aktivitätsmustern, Wanderverhalten, Parasitenbefall, der mikrobiellen Darmflora und Konzentrationen von fäkalen Glucocorticoid-Metaboliten (fGCMs) untersucht.

Keines der Tiere zeigte Anzeichen einer Erkrankung und es gab nur wenige Hinweise darauf, dass das Sozialleben der Spezies Auswirkungen auf die Gesundheit der Individuen hat. Genauer gesagt hatten Gruppengröße und soziale Interaktionen keinen Einfluss auf Verhaltens- oder physiologische Parameter. Variationen in Aktivitätsmustern und Wanderverhalten zwischen den Gruppen reflektierten vermutlich Anpassungen an Unterschiede in den Mikrohabitaten, sorgten aber nicht für Veränderungen in fGCMs oder Parasitenbefall, was darauf hinweist, dass diese Anpassungen keine gesundheitlichen Auswirkungen hatten. Die Nähe zu Gruppenmitgliedern vereinfachte die Übertragung von Mikroorganismen, was darauf schließen lässt, dass das Leben in Gruppen in Bezug auf Parasitenübertragungen nachteilig sein kann, wohingegen der Austausch nutzbringender Darmbakterien gesundheitsfördernde Auswirkungen haben könnte. Die Ergebnisse dieser Dissertation implizieren, dass verschiedene Aspekte des Gruppenlebens in Verreaux's Larvensifakas eingeschränkte Konsequenzen für die Gesundheit der Tiere haben, welche durch soziale Nähe, nicht aber durch soziale Interaktionen verursacht werden. 
Schlussendlich hängt das Ausmaß der gesundheitsbezogenen Konsequenzen von Sozialität von den artspezifischen Aspekten des jeweiligen Sozialsystems ab. In Arten mit hoch-kompetitiven Sozialgefügen und höheren Raten an agonistischen und freundlichen Interaktionen zwischen Individuen, könnte es beispielsweise zu größeren Variationen in energetischem und sozialem Stress kommen und Möglichkeiten der Übertragung von Mikroorganismen könnten zunehmen. Im Gegensatz dazu wären die gesundheitsbezogenen Konsequenzen von Sozialität in Arten mit weniger kompetitiven Regimen und niedrigeren Interaktionsraten, wie es zum Beispiel bei Verreaux's Larvensifakas der Fall ist, vermindert. Zur Anknüpfung an diese Thematik sind weitere Freilandstudien mit verschiedenen Sozialsystemen im ökologisch relevanten Kontext nötig, um eine Verständniserweiterung der komplexen und voneinander abhängigen Faktoren zum Zusammenhang von Sozialität und Gesundheit zu ermöglichen. 


\section{Chapter 1}

\section{General Introduction}

\subsection{Towards Understanding the Evolution of Sociality}

When, how and why did animals begin to live in groups? Why do some species form larger groups than others? What are the trade-offs of being social? The evolutionary switch from solitary to group living represents one of the major transitions in evolution as it had fundamental consequences for animals' life histories (Szathmáry and Smith, 1995). A better understanding of the basic principles and consequences of this transition yields novel practical applications for various scientific disciplines, like immunology, neurology, genetics, ecology or computer science, and will shed more light on the history and evolution of humans' complex societies. However, despite the great deal of scientific progress that has been made in recent years, there remain many questions surrounding the evolution of sociality to be answered.

\section{The Troublesome Semantics}

Even though the mechanisms and factors that governed the transition from solitary to group living have been studied in depth (Krause and Ruxton, 2002; Ward and Webster, 2016), research still struggles to amend a unified conceptual framework applicable to all disciplines and taxa. This is partly due to the missing consent on the use of semantics (Costa and Fitzgerald, 2005, 1996; Krause and Ruxton, 2002). For example, there is disagreement on the definition and use of the term "social". Some researchers refer to animals as social if their range of behaviours involves interactions with conspecifics (Brown, 1975), while others speak of an interdependence of conspecifics that forage together and thereby affect each other's energetic gains and losses (Giraldeau and Caraco, 2018). To make things more complicated, there is an increasing amount of vertebrate literature assigning degrees of sociality to certain species by using terms like "highly social" or "socially complex" without providing explicit definitions (Kappeler et al., 2015). Meanwhile, studies of invertebrates apply categories to sociality, e.g. "semi-social", "parasocial" or "quasi-social", which cannot be easily applied to vertebrates (Costa and Fitzgerald, 2005). Throughout this thesis, I will apply the term "social" in the sense of Tinbergen's definition: "An animal is called social when it strives to be in the neighbourhood of fellow members of its species when performing some, or all, of its instinctive activities" (Tinbergen, 1951).

Following on from this, there also exist numerous definitions for the terms "group" and "social group" (see Krause and Ruxton, 2002; Ward and Webster, 2016). The difficulty lies in finding definitions that applies to all species. For example, some animals form short-term aggregations in response to temporally or spatially clumped resources, while others may live permanently in close proximity to conspecifics. Here, I consider a species as group living, if two or more conspecifics spent a significant 
amount of time in proximity close enough to allow continuous information exchange (modified from Pitcher (1983)). I refer to species as living in social groups, if they additionally interact with their group members "to a distinctly greater degree than with other conspecifics" (Struhsaker, 1969; Wilson, 1975).

\section{Why Become Social?}

The majority of animal species live solitary, which is considered the ancestral condition of animals' social systems (Lukas and Clutton-Brock, 2013; Majolo and Huang, 2018). Permanent co-residency with conspecifics elicits competition for resources and mates and stable aggregations of animals only occur if competition among individuals can be overcome by the benefits of gregariousness (Krause and Ruxton, 2002; Ward and Webster, 2016). Such benefits are generally related to predation risks, access to food and access to mating opportunities (Alexander, 1974; Hamilton, 1971; van Schaik et al., 1983). Taxa in which benefits of gregariousness outweighed its costs, eventually formed groups of different sizes, compositions and stability, leading to the emergence of a wide array of diverse social systems ranging from small pair-bonded units to large aggregations (Ward and Webster, 2016). However, there remains little knowledge about the consequences of group living and how these consequences may vary across social systems or individuals within the same group (Kappeler et al., 2015; Silk, 2007).

\section{Health-Related Costs and Benefits of Sociality}

There is accumulating evidence on a wide range of taxa linking aspects of sociality to differential survival and reproductive success (Archie et al., 2014; Bilde et al., 2007; Cameron et al., 2009; House et al., 1988; Jungwirth and Taborsky, 2015; Meunier, 2015; Oli and Armitage, 2003; Ostner and Schülke, 2018; Silk, 2007; Verbrugge, 1979). For example, humans with stronger social relationships live longer (Holt-Lunstad et al., 2010), horses, baboons and dolphins that are better socially integrated benefit from higher birth rates and offspring survival (Cameron et al., 2009; Frère et al., 2010; McFarland et al., 2017; Silk et al., 2003), and colony size in social spiders is positively linked to offspring survival but decreases individual reproductive success (Avilés and Tufiño, 1998). While the link between sociality and fitness is well established and reproducible, the behavioural and physiological mechanisms by which these links are exerted remain not well understood. One mediator for the fitness consequences of sociality is individual health (Kappeler et al., 2015), which can be both, improved or worsened by different aspects of group living.

\section{Defining "Health"}

To understand and compare health consequences of sociality, it is necessary to first find a broad agreement on how to define health. This has, however, proven difficult in the past and debates have been ongoing for centuries, resulting in numerous proposals (Chatfield, 2018; van de Belt et al., 2010). The most accepted and commonly used definition was provided by the WHO (World Health Organisation), 
which defines health as "a state of complete physical, mental and social wellbeing and not merely the absence of disease or infirmity" (World Health Organization, 1948). However, this definition has received much criticism due to its static nature, i.e. health as a state, and its requirement for completeness, which would be hardly ever fulfilled by anyone (Jambroes et al., 2016; Smith, 2008). Other objections concerning changes in disease patterns and societies' demography within the last 50 years or discrepancies between the terms "health" and "wellbeing" were brought up (reviewed in Chatfield, 2018; Huber et al., 2011). A new proposal for conceptualising health was presented in 2011, defining health as "the ability to adapt and to self-manage' when facing physical, mental, and social challenges" (Huber et al., 2011). Accordingly, health is considered more dynamic as it describes individuals' capacities of maintaining physiological homeostasis. This way health can be evaluated with a set of dynamic and interrelated features, which offer valuable information about various aspects of individual condition. It is therefore that I will apply the term "health" in the sense of this definition.

\section{Parasites and Diseases}

One of the major health-related costs of group living constitutes the transmission and spread of parasites and diseases (Alexander, 1974; Freeland, 1976). Parasites can be transmitted via two pathways: directly, e.g. through physical contact, or indirectly, e.g. through shared environments (White et al., 2017). Physical contact to conspecifics presumably increases opportunities for direct transmissions, which is why transmission rates are generally expected to increase with group size or group density (Altizer et al., 2003; Nunn et al., 2003; but see Rifkin et al., 2012). Characteristics of species' social networks can provide more information about groups' structures and animals' social relationships (Wey et al., 2008) and potentially enable a better understanding of transmission dynamics than measures of group size (Poulin, 2018). For example, in female Japanese macaques (Macaca fuscata yakui) central position within groups was correlated with increased nematode infections (Maclntosh et al., 2012), and type and direction of physical contacts explained helminth prevalence in brown spider monkeys (Ateles hybridus) (Rimbach et al., 2015) and the spread of infectious fungi in garden ant colonies (Lasius neglectus) (Theis et al., 2015). Infestation with environmental parasites might be facilitated if increased host density leads to higher environmental contamination and, therefore, increased contact with infectious parasite stages (Chapman et al., 2005; Ezenwa, 2004; Poirotte et al., 2016). The transmission of sexually transmitted diseases is expected to increase with promiscuity, constituting another example for the exploitation of host social behaviour through pathogens (Nunn et al., 2014; Thrall et al., 2000).

Group living animals also embark strategies to defend themselves against parasites and diseases (Schmid-Hempel, 2017). For example, allogrooming in primates can reduce ectoparasite load (Akinyi et al., 2013; Duboscq et al., 2016; Nunn and Altizer, 2006; Sánchez-Villagra et al., 1998). In garden ants, grooming of individuals with fungal infections can be mutually beneficial as the groomee gets alleviated 
from the infection, while the groomer receives a low-level fungal infection, promoting immunisation (Konrad et al., 2012). This "social immunisation" (Cremer et al., 2007; Konrad et al., 2012; Traniello et al., 2002) has also been observed in vertebrates, where low-level exposures to pathogens through proximity to conspecifics lead to the development of adaptive immunity (Hart, 2011).

\section{Immune System Functionality}

The immune system provides a powerful defence against parasites and diseases that functions via multiple pathways but comes with high energetic costs (Sheldon and Verhulst, 1996; Straub et al., 2010). Individuals living in groups frequently compete over food and mates, which can impose energetic constraints, especially to those with inferior competitive abilities (Lane et al., 2010; Metcalfe, 1986; van Schaik et al., 1983). If energetic constraints persist, individuals might not be able to allocate sufficient energy to their immune system, resulting in reduced immunocompetence and increased susceptibility to infectious agents (Kappeler et al., 2015). For example, specific ranks within social hierarchies can impose varying energetic requirements to individuals (Emery Thompson et al., 2010). This might explain why rank-related differences in health are partly associated with rank-related differences in immune system functionality (Fairbanks and Hawley, 2012; Habig et al., 2018; Habig and Archie, 2015; Snyder-Mackler et al., 2016). Social stress can also impair immune functions, as negative life events and social isolation in humans, and increased social conflict in animals are associated with increased vulnerability to diseases (Cohen et al., 2012, 1997; Holt-Lunstad et al., 2010; McEwen, 2012; McEwen et al., 1997; Widom, 1999).

\section{Stress and Glucocorticoids}

Links between social stress and individual's immune responses are presumably mediated via the activities of the hypothalamic-pituitary-adrenocortical (HPA) system, which releases glucocorticoids (GCs) - one of the most studied adrenal hormones (Creel et al., 2013; Defolie et al., 2019; Goymann and Wingfield, 2004; Sapolsky et al., 2000). GCs' main function lies in energy balance modulation, but they are also involved in a myriad of other physiological processes, including their role within the body's complex physiological stress response (Beehner and Bergman, 2017; Higham, 2016). GCs get released into the body within minutes to hours after an acute stressor triggered the activation of the HPA axis (Sapolsky et al., 2000) and can impact behaviour and metabolic, reproductive, and immune systems (Landys et al., 2006; Romero et al., 2009; Sapolsky et al., 2000). Their effects can be both, immunoenhancing and immunosuppressive, e.g. through regulation of inflammatory processes (Besedovsky et al., 1986; Dhabhar, 2009; Elenkov and Chrousos, 1999) or by triggering changes in blood leukocyte distributions (Dhabhar et al., 1996; Fauci and Dale, 1974). Some studies found associations between increased concentrations of GCs and slower rates of wound healing (Archie et al., 2012; French et al., 2006; Walburn et al.; but see 2009 Archie, 2013). 
Due to their impact on immune functions, their ubiquity across vertebrates and multiple noninvasive sampling possibilities (e.g. via faeces, urine, saliva), studies in stress biology rely more and more on GC measures as biomarkers for stress (McCormick and Romero, 2017). However, in the first place, GCs are metabolic hormones that provide energy to the body when required and reflect energetically demanding periods, e.g. during different reproductive stages or circannual variation in temperatures and food availability (Beehner and Bergman, 2017). An increase in GCs is, therefore, not necessarily associated with a stress response, which is why GC concentrations do not constitute reliable indicators of individual stress levels (Beehner and Bergman, 2017; Higham, 2016; MacDougall-Shackleton et al., 2019). Nevertheless, measures of GCs allow researchers to measure the energetic costs of sociality, which is why they provide a valuable resource for understanding the sociality-health nexus.

In numerous studies, different aspects of group living have been linked to variation in GC concentrations (reviewed in Creel et al., 2013; Raulo and Dantzer, 2018). For example, in mammals, measures of GCs were positively correlated to population density or group size (Boonstra and Boag, 1992; Dantzer et al., 2013; Foley et al., 2001; Raouf et al., 2006). Differences in social status can be accompanied by varying energetic constraints and, thus, changes in GC concentrations (Abbott et al., 2003; Emery Thompson et al., 2010; Gesquiere et al., 2011). However, whether higher or lower ranks are energetically more costly and trigger GC secretion depends on different aspects of a species social system, like social structure, social organisation or mating system (Beehner and Bergman, 2017; Sapolsky, 2005). Moreover, agonistic interactions, social isolation and social instability (Cavigelli et al., 2003; Culbert et al., 2018; Dantzer et al., 2017; Girard-Buttoz et al., 2009; van Meter et al., 2009) are often linked to increased GC outputs, while opposite effects are reported for affiliative interactions (Raulo and Dantzer, 2018).

GCs exert powerful effects on animals' behaviours and physiology, and links between GC concentrations and social behaviours are well established. However, there exist a variety of intrinsic and extrinsic factors that can trigger and modulate GC secretions and ought to be controlled for in endocrinological research (Higham, 2016; Rimbach et al., 2013). One of these confounding factors is the gut microbiome (Foster et al., 2017).

\section{Bacterial Gut Microbiota}

Humans and animals harbour trillions of bacteria within their gastrointestinal tracts that play major roles for their health and fitness. Gut bacteria enable digestion, produce vitamins from the diet, they influence immune system development and protect their hosts from infection (Kinross et al., 2011; Ley et al., 2005; Turnbaugh et al., 2006a). Especially within the last decade, a basic understanding of the functions microbial communities have in human health and diseases has been established (Cresci and Bawden, 2015), while their role in other animals has yet to be investigated in depth. 
The assemblage of the gut microbiota can be influenced by numerous intrinsic factors, like age (Amato et al., 2014; Pafčo et al., 2019), sex (Dominianni et al., 2015; Ren et al., 2016), or host genotype (Dąbrowska and Witkiewicz, 2016; Degnan et al., 2012a; Kovacs et al., 2011); and extrinsic factors, like parasites (Boutin et al., 2013; Morton et al., 2015) or diet (Greene et al., 2018; Youngblut et al., 2019). Social behaviours can also modify gut microbiome composition and diversity (Ezenwa et al., 2012; Gilbert, 2015; Lombardo, 2008; Montiel-Castro et al., 2013) as shown in various taxa, ranging from insects (Koch and Schmid-Hempel, 2011) and birds (Kulkarni and Heeb, 2007) to carnivores (Theis et al., 2015) and primates (Clayton et al., 2018). Group living animals often share more similar gut microbial communities with group members than with outsiders (Bennett et al., 2016a; Chiyo et al., 2014; Grieneisen et al., 2017; Raulo et al., 2017) and studies in primates found rates of social interactions to covary with gut microbiome similarity (Moeller et al., 2016; Tung et al., 2015).

There are multiple bidirectional connections that link the gut microbiota with its host's central nervous system via endocrine, immune and neural pathways. These connections are summarised in the term "gut-brain axis" (Foster et al., 2017; Grenham et al., 2011; Montiel-Castro et al., 2013). A growing number of studies suggests that gut bacteria play a fundamental role in regulating their host's immune system, physiological stress response and other important physiological functions and, thus, have profound impact on host health. For example, HPA axis reactivity can be influenced through experimental alterations of gut microbial communities (Carabotti et al., 2015; Neufeld et al., 2011; Sudo, 2014) and increases in GC concentrations have been linked to decreased microbial diversity or changes in the abundance of certain microbial phyla in gorillas (Gorilla gorilla gorilla) (Vlčková et al., 2018) and yellowlegged gulls (Larus michahellis) (Noguera et al., 2018). Similarly, a recent study on grey squirrels (Sciurus carolinensis) found links between various measures of HPA activity, including GC metabolites, and gut microbiome composition and diversity (Stothart et al., 2019). Moreover, cases of dysbiosis - an imbalance of the gut microbial community - are associated with lower serum immunoglobulin levels, decreased lymphocytes (Round and Mazmanian, 2009) and various intestinal, metabolic and central nervous systemrelated disorders (Rinninella et al., 2019).

Various aspects of sociality can benefit individual health via the social transmission of gut bacteria. More precisely, socially transmitted bacteria can contribute to pathogen resistance and stimulate host immunity (Koch and Schmid-Hempel, 2011; Montiel-Castro et al., 2013). Commensal microbes might also outcompete pathogens for resources or produce by-products that inhibit pathogens (Abt and Pamer, 2014; Ezenwa et al., 2016). Moreover, frequent social transmission may increase microbial diversity, which is associated with improved health (Browne et al., 2017).

Altogether, there is increasing evidence that social interactions are associated with health-related trade-offs. However, inter- and intraspecific variability in the patterns that link various health-indicators with various aspects of sociality remains high (Kappeler et al., 2015). One reason for this is that 
physiological parameters of health, like parasites, GCs and microbiomes, are highly interrelated and studies that explicitly examine multiple health indicators simultaneously in order to gain a comprehensive understanding of their interactions are still missing. Moreover, most knowledge on the sociality-health nexus is based on clinical and laboratory studies, which is why the understanding of the relative importance, underlying mechanisms, dynamics and interactions of social factors on individual health in wild animal populations is still relatively poor (Gesquiere et al., 2011; Huffman and Chapman, 2009; Nunn and Altizer, 2006; Silk et al., 2010).

\section{The Indispensable Value of Comprehensive Field Research}

Within the last decades, laboratory studies enabled major discoveries in and created the fundament for today's parasitological, endocrinological and microbial research. However, studies in artificial environments are unable to replicate the manifold factors animals face under natural conditions, which is why field studies are of great importance when studying links between sociality and health. Various environmental factors can influence animal physiology and behaviours, such as seasonal changes in temperatures and food availability (Bekoff et al., 1984; Bronikowski and Altmann, 1996; Iwamoto and Dunbar, 1983), habitat quality (Franklin et al., 2000; Strandburg-Peshkin et al., 2017), or anthropomorphic disturbances (Junge et al., 2011; Marty et al., 2019; van Meter et al., 2009). Inclusion of potentially confounding factors increases the general understanding of the links between social behaviours and their physiological consequences in wild animals. Moreover, great progress has been made on the application of non-invasive techniques to collect and analyse samples without disturbing animals' natural behaviours. This progress allows studies to gain greater ecological validity and to widen the range of species that can be tested under natural conditions (Higham, 2016).

\subsection{Studying Lemurs}

Without nonhuman primates (hereafter referred to as primates), humans would be - phylogenetically speaking - isolated from the rest of the animal kingdom and attempts to study human social evolution would be limited to testing theories based on general principles applicable to all mammals. Fortunately, however, there exist over 500 different primate species which show high diversity in social complexity (Mitani et al., 2012). At least 79 \% of primate species are group living (Silk and Kappeler, 2017) and the manifold nature of primates' social systems makes them particularly useful for interspecific comparative studies on the evolution of group living and the several new health-and fitness-related costs and benefits that derived from this evolution.

Lemurs represent an especially interesting primate radiation for studies on the evolution of sociality. They are endemic to the island of Madagascar where they evolved from one common ancestor independently from anthropoid primates (Mitani et al., 2012). Lemur groups show three major 
differences to their continental relatives. First, they are smaller, most likely because of adaptations to feeding competition (Kappeler and Heymann, 1996; Wright, 1999). Second, they display fairly even sex ratios, which might be explained through their relatively recent evolutionarily transition to group living (Kappeler et al., 2009a; van Schaik and Kappeler, 1996). Third, not all types of social organisations that can be found in anthropoids are represented in lemurs, e.g. there are no groups with single breeding males ("harems") or females ("polyandrous groups") and their groups never comprise multiple social layers as can be found, for example, in baboons (Mitani et al., 2012). Therefore, examinations of consequences of sociality in lemurs will prove useful since they provide new insights into the similarities and dissimilarities of health and fitness consequences of social behaviours between different primate radiations, which will help to explain general principles of the evolution of group living.

\section{Verreaux's Sifakas as Study System}

In this thesis, I studied health-consequences of group living in a wild population of Verreaux's sifakas (Propithecus verreauxi) at Kirindy Forest, in western Madagascar. There are nine different species within the genus Propithecus (family Indriidae), which are distributed throughout the whole island (Figure 1). All of them are group living, arboreal and belong to the larger representatives among lemurs (Mittermeier et al., 2008). Verreaux's sifakas are diurnal, have a frugi-folivorous diet, inhabit the dry forests of southern and south-western Madagascar and live in small multi-male multi-female groups ranging in size from 2 to 12 animals (Jolly et al., 1982; Kappeler and Fichtel, 2012; Leimberger and Lewis, 2015; Sussman et al., 2012). This species was one of the first lemurs to be studied in the wild (Jolly, 1966; Richard et al., 2002) and to date much research has been conducted on their social organisation, life histories, mating tactics, and intergroup relations (Benadi et al., 2008; Erkert and Kappeler, 2004; Kappeler and Fichtel, 2012; Koch et al., 2016a; Markham and Gould, 2018; Springer et al., 2016).

Previous work on the population at Kirindy Forest suggests that Verreaux's sifakas are well suited for studying health consequences of sociality because:

1) Measures of faecal glucocorticoid metabolites (Fichtel et al., 2007) and parasitism (Springer and Kappeler, 2016) are well established and validated, and GPS tags have been successfully applied to record long-term ranging patterns (Koch et al., 2016a).

2) With 7 habituated, adjacent groups (Figure 2), the population in Kirindy Forest is at the upper end in terms of study groups compared to field research in other primates (see e.g. Fürtbauer et al., 2014; Ganas and Robbins, 2005; Markham et al., 2015; Snaith and Chapman, 2008; Stevenson and Castellanos, 2000). 


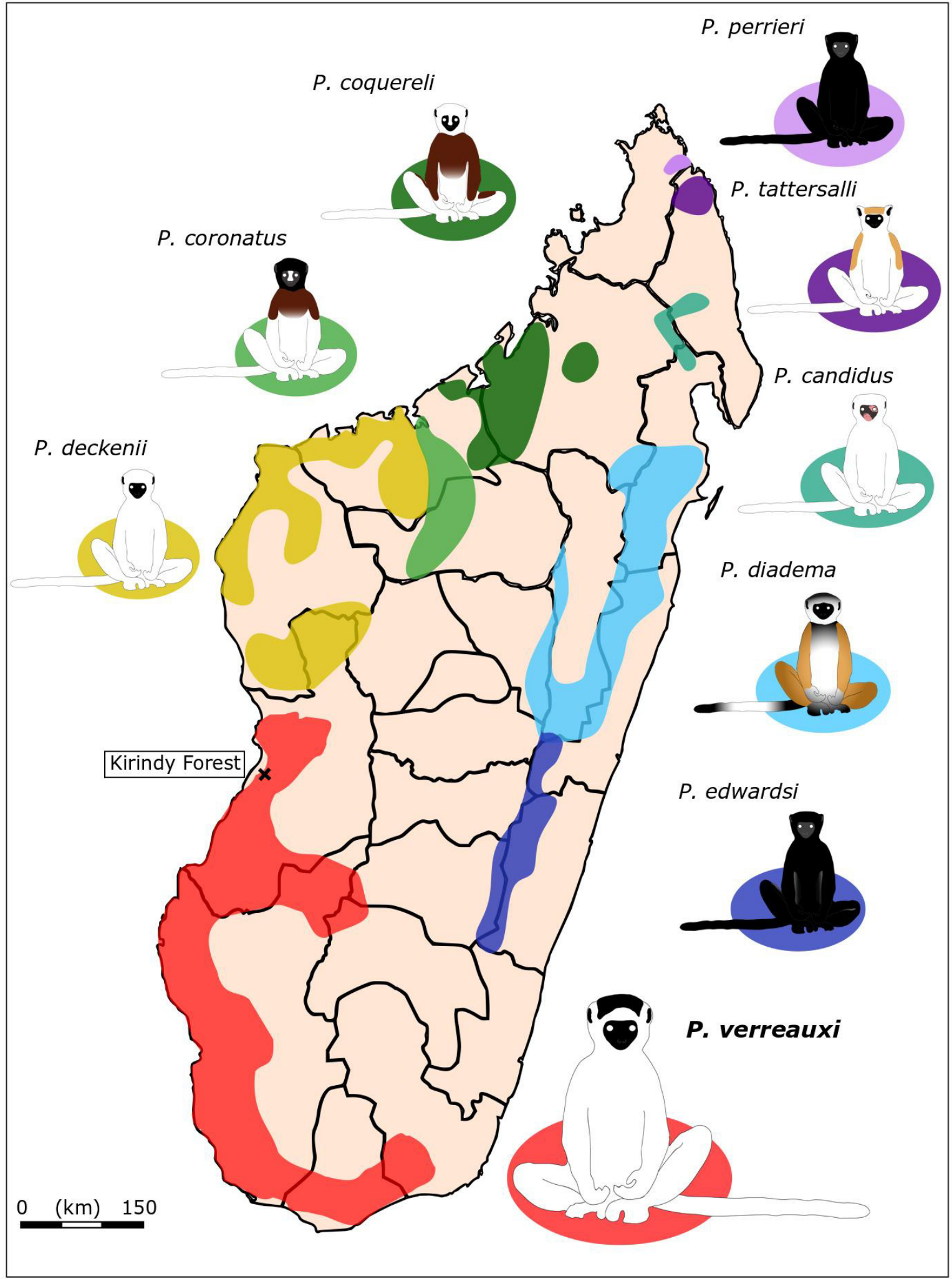

Figure 1 Illustration of the distribution of the nine different Propithecus species (based on www.iucnredlist.org, access date 01/12/2019). The map was created by Vemaps.com and modified by Katja Rudolph 


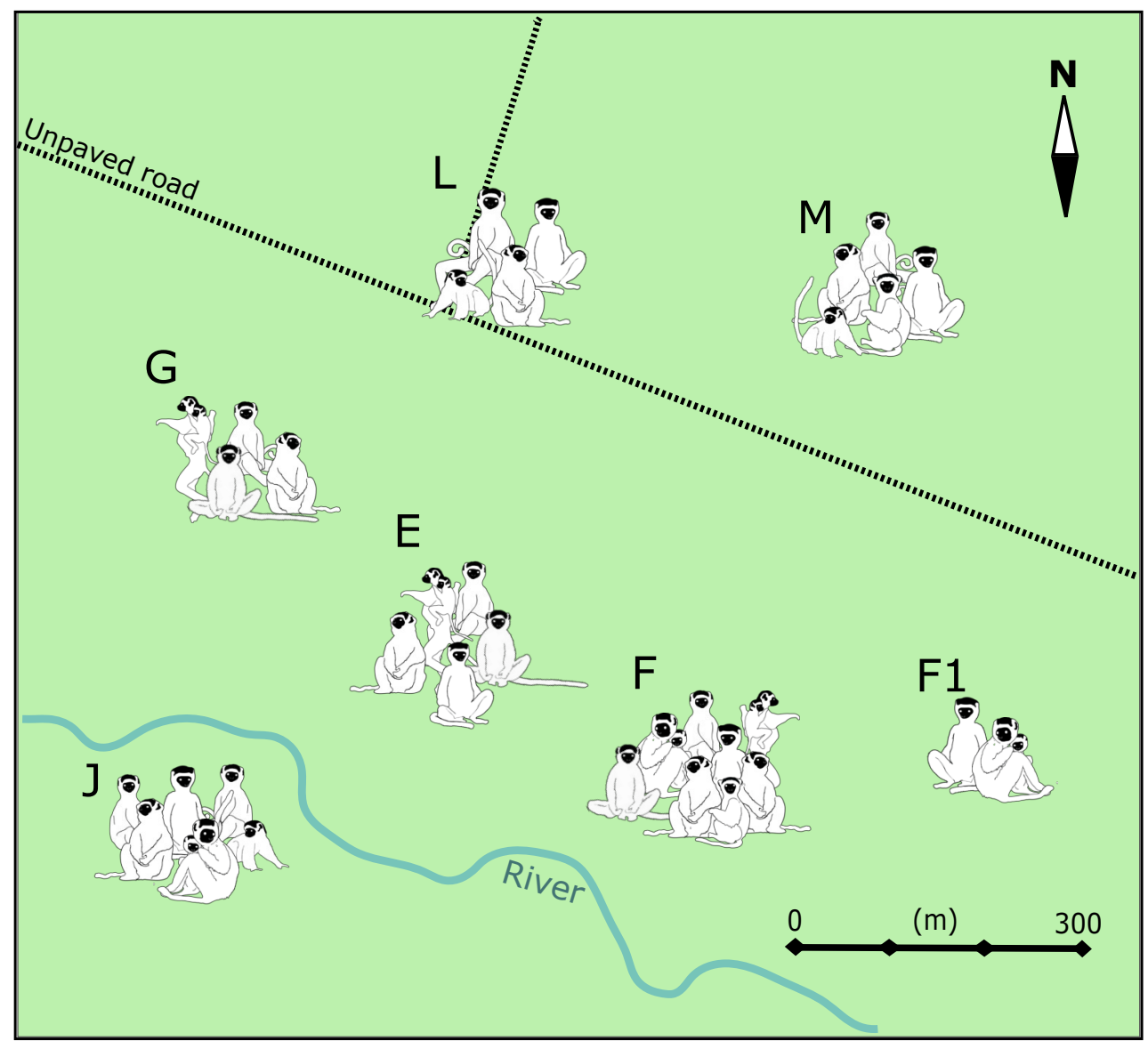

Figure 2 Schedule of the study site with the location of seven study groups at Kirindy Forest, Madagascar. Letters indicate labels of the groups

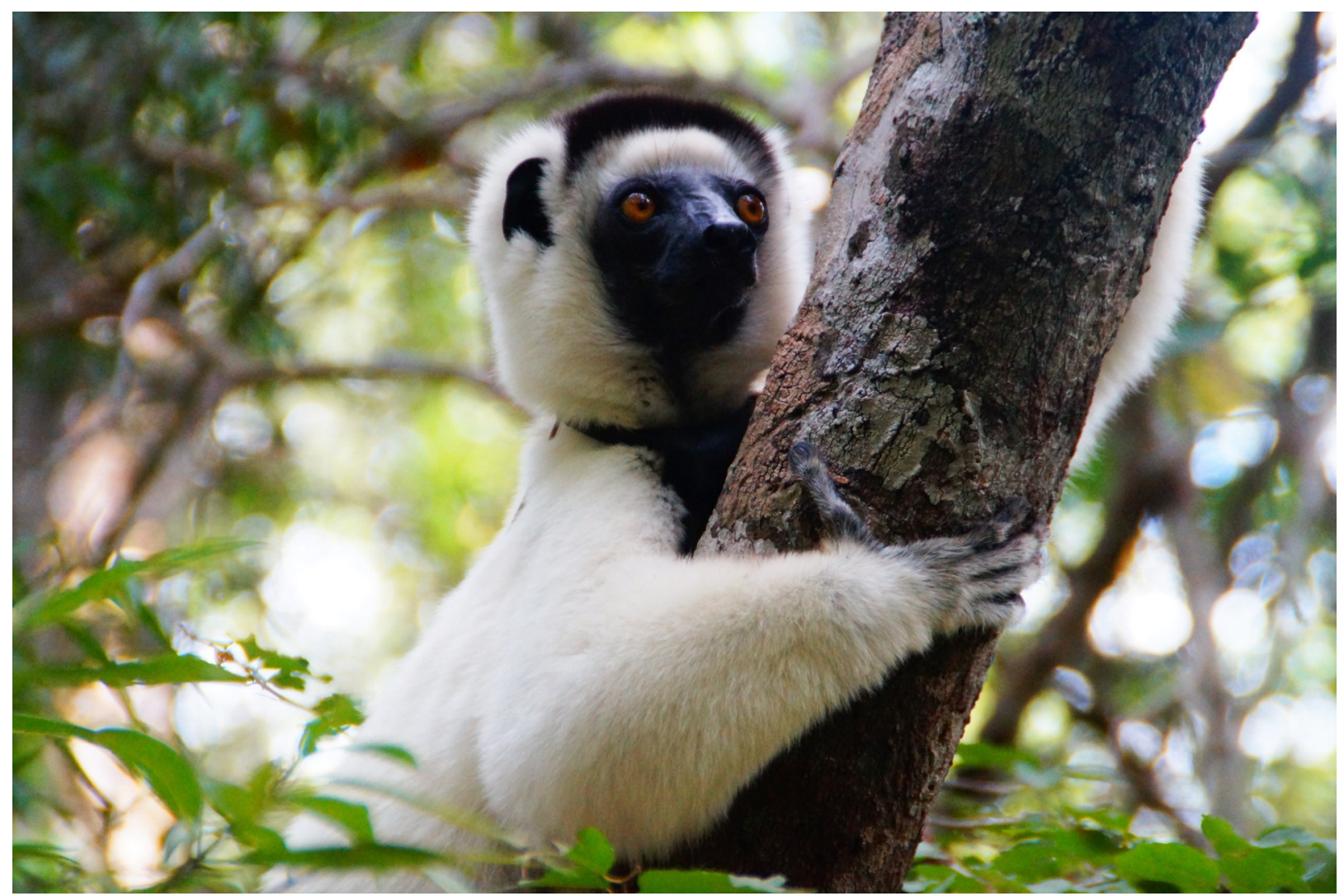

Figure $3 \mathrm{An}$ adult female Verreaux's sifaka with radio collar 
3) Most knowledge that has been gained on the sociality-health nexus in wild primate populations derives from species living in large groups with up to $>50$ individuals, e.g. yellow and chacma baboons (Papio cynocephalus and P. hamadryas ursinus) (Crockford et al., 2008; Markham et al., 2015; Silk et al., 2010), chimpanzees (Pan troglodytes) (Wittig et al., 2016) or Japanese macaques (Duboscq et al., 2016), while species living in smaller groups are underrepresented. However, interspecific variation in health and fitness consequences of group living may crucially depend on group size (Chapman and Chapman, 2000; Wrangham et al., 1993).Investigations of the sociality-health nexus in a lemur species that lives in small groups will, therefore, provide new insights into the impact of group size on health-related consequences of sociality.
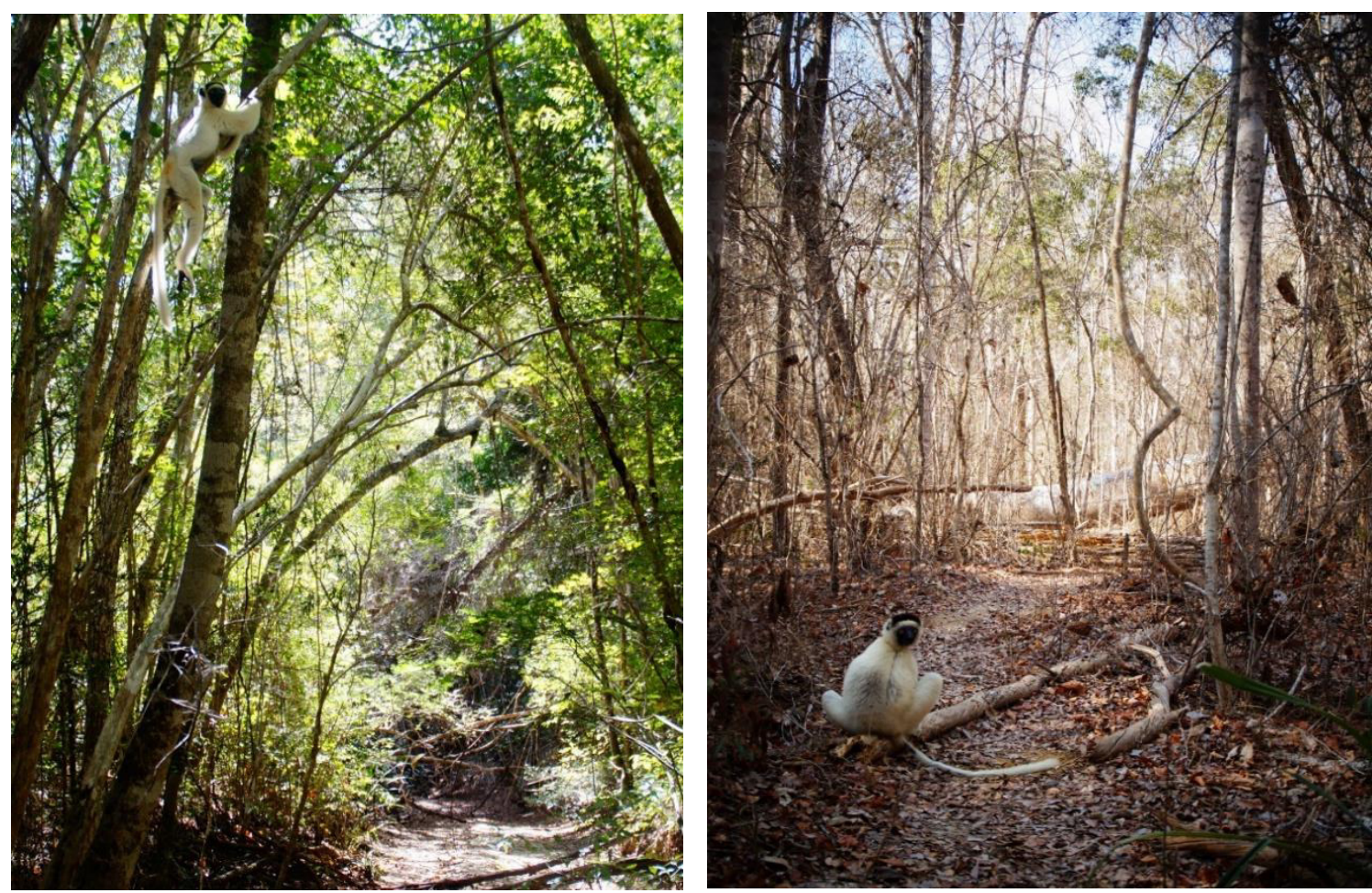

Figure 4 Kirindy Forest during the wet (left) and the dry season (right). (c) Katja Rudolph

4) Kirindy Forest is subject to pronounced seasonality, with a long, cool dry season (April to October) and a hot wet season (November to March) (Kappeler and Fichtel, 2012; Figure 4). Since the study population is part of an ongoing long-term study (Kappeler and Fichtel, 2012), data on monthly precipitation, temperatures and phenology are collected in great detail, allowing to control for various ecological confounding factors.

\subsection{Objectives of This Thesis}

The general aim of my thesis is to illuminate components and links of the sociality-health nexus in a wild population of Verreaux's sifakas. Therefore, I examine associations of various aspects of sociality within and between groups with an array of behavioural, physiological and ecological variables, some of which function as indicators for animals' physical condition. Over two consecutive years and with the help of 
field assistants, I collected and analysed a comprehensive data set comprising ranging, feeding and social behaviours and measures of faecal glucocorticoid metabolites (fGCMs), parasites and bacterial gut communities.

In study 1 (chapter II), I test predictions of the ecological constraints and the optimal group size hypothesis, which link group size to condition and health. I investigate the impact of group size and group membership on daily travel distances, home range sizes, daily activities, fGCMs and parasite richness while controlling for the potentially confounding effects of seasonality and habitat quality.

After examining fGCM concentrations between groups in study 1 , I focus in study 2 (chapter III) on $\mathrm{fGCM}$ variations between individuals. To better understand the dynamics and determinants of fGCMs, I investigate potential associations with social interactions, group composition, rank, reproductive state, vigilance, scratching, diet, food availability and daily temperature differences - factors that are all suggested to impact GC outputs in vertebrate species.

In study 3 (chapter IV), I investigate the drivers of between-group variation in bacterial gut communities. An increasing number of studies in a wide range of taxa, including Verreaux's sifakas, finds group members to share more similar gut bacterial communities than individuals living in different groups (Grieneisen et al., 2017; Koch and Schmid-Hempel, 2011; Lax et al., 2014; Springer et al., 2017; Theis et al., 2012). However, the drivers of this microbial convergence among group members remain little understood. I combine data on social interactions, maternal relatedness, diet, habitat structure, habitat overlap and seasonality with measures of gut microbial communities, derived from $16 \mathrm{~S}$ rRNA sequencing, to evaluate the relative contribution of social, genetic and ecological factors to shaping groups' distinct gut microbiomes. 


\title{
Chapter 2
}

\author{
Study I
}

\section{One Size Fits All? Relationships Among Group Size, Health, and Ecology Indicate a Lack of an Optimal Group Size in a Wild Lemur Population}

with Claudia Fichtel ${ }^{1,3}$, Dominik Schneider ${ }^{4}$, Michael Heistermann ${ }^{5}$, Flávia Koch ${ }^{1}$, Rolf Daniel ${ }^{4}$,

and Peter M. Kappeler ${ }^{1,2,3}$

${ }^{1}$ Behavioral Ecology \& Sociobiology Unit, German Primate Center, Leibniz Institute for Primate Research, Göttingen, Germany

${ }^{2}$ Dept. Sociobiology /Anthropology, Johann-Friedrich-Blumenbach Institute of Zoology and Anthropology, University Göttingen, Göttingen, Germany

${ }^{3}$ Leibniz Science Campus "Primate Cognition", Göttingen, Germany

${ }^{4}$ Genomic and Applied Microbiology and Göttingen Genomics Laboratory, Institute of Microbiology and Genetics, University Göttingen, Göttingen, Germany

${ }^{5}$ Endocrinology Laboratory, German Primate Center, Leibniz Institute for Primate Research, Göttingen, Germany

This chapter is published in

Behavioral Ecologogy and Sociobiology (2019) 73: 132

https://doi.org/10.1007/s00265-019-2746-0 


\title{
One size fits all? Relationships among group size, health, and ecology indicate a lack of an optimal group size in a wild lemur population
}

\author{
Katja Rudolph ${ }^{1,2,3}$ - Claudia Fichtel ${ }^{1,3} \cdot$ Dominik Schneider $^{4} \cdot$ Michael Heistermann $^{5} \cdot$ Flávia Koch $^{1} \cdot$ Rolf Daniel $^{4}$. \\ Peter M. Kappeler ${ }^{1,2,3}$
}

Received: 19 April 2019 /Revised: 19 August 2019 / Accepted: 21 August 2019

(C) The Author(s) 2019

\begin{abstract}
Group size is a key component of sociality and can affect individual health and fitness. However, proximate links explaining this relationship remain poorly understood, partly because previous studies neglected potential confounding effects of ecological factors. Here, we correlated group size with various measures of health while controlling for measures of seasonality and habitat quality, to explore trade-offs related to group living in a mainly folivorous primate-Verreaux's sifakas (Propithecus verreauxi). Over a course of 2 years, we studied 42 individuals of 7 differently sized groups (range 2-10) and combined measures of faecal glucocorticoid metabolites ( $n>2300$ samples), parasitism ( $n>500$ samples), ranging and activity patterns, together with estimates of habitat quality (measures of $\sim 7000$ feeding trees). None of our measures was correlated with group size, while seasonality, but not habitat quality, impacted almost all examined variables. We conclude that group size alone might be insufficient to explain patterns in the sociality-health nexus or that the small range of group sizes in this species does not induce effects suggested for species living in larger groups. An optimal group size balancing the advantages and disadvantages of living in differently sized groups may not exist for Verreaux's sifakas. Our results do not support predictions of the ecological constraints hypothesis or the optimal group size hypothesis as they may only account for species limited in group size by ecological factors-a condition that may not apply to the majority of folivorous mammals, which seem to be limited by social factors.
\end{abstract}

Communicated by M. A. van Noordwijk

Electronic supplementary material The online version of this article (https://doi.org/10.1007/s00265-019-2746-0) contains supplementary material, which is available to authorized users.

\author{
Katja Rudolph \\ KRudolph@dpz.eu \\ Claudia Fichtel \\ Claudia.fichtel@gwdg.de \\ Dominik Schneider \\ dschnei1@gwdg.de \\ Michael Heistermann \\ MHeistermann@dpz.eu \\ Flávia Koch \\ biokoch@gmail.com \\ Rolf Daniel \\ rdaniel@gwdg.de \\ Peter M. Kappeler \\ pkappel@gwdg.de
}

1 Behavioral Ecology \& Sociobiology Unit, German Primate Center, Leibniz Institute for Primate Research, Kellnerweg 4, 37077 Göttingen, Germany

2 Department Sociobiology/Anthropology, Johann-Friedrich-Blumenbach Institute of Zoology and Anthropology, University Göttingen, Kellnerweg 6, 37077 Göttingen, Germany

3 Leibniz Science Campus "Primate Cognition", Göttingen, Germany

4 Genomic and Applied Microbiology and Göttingen Genomics Laboratory, Institute of Microbiology and Genetics, University Göttingen, Grisebachstraße 8, 37077 Göttingen, Germany

5 Endocrinology Laboratory, German Primate Center, Leibniz Institute for Primate Research, Kellnerweg 4, 37077 Göttingen, Germany 


\section{Significance statement}

Group size is a key component of group living and can crucially impact individual health. Ecological variables may modulate this relationship, but they were often neglected in previous studies. To better understand the links between sociality and health, we, for the first time in a mammal, simultaneously examined variation in ranging patterns, daily activities, glucocorticoid concentrations, and parasitism as a function of group size and under consideration of measures of seasonality and habitat quality in wild Verreaux's sifakas (Propithecus verreauxi). Group size had no impact on individual health indicators, while seasonal variation in food availability and temperature differences, but not habitat quality, affected the majority of variables. We demonstrate strong impacts of environmental factors on socio-ecological traits and conclude that group size on its own might be insufficient to explain patterns in the sociality-health nexus.

Keywords Optimal group size $\cdot$ Glucocorticoids $\cdot$ Parasites $\cdot$ Daily travel distance $\cdot$ Habitat quality $\cdot$ Propithecus verreauxi

\section{Introduction}

Group size has been identified as one key aspect of sociality that influences an individuals' condition and health (Altizer et al. 2003; Borries et al. 2008; Markham et al. 2015; Ezenwa et al. 2016). According to the ecological constraints hypothesis, feeding competition, parasite infestations, and energy expenditure should be higher in larger groups, whereas smaller groups face higher per capita predation risk and disadvantages during competitive encounters with larger groups (Wrangham et al. 1993; Chapman and Chapman 2000, but see Koch et al. 2016). The optimal group size hypothesis posits that intermediate-sized groups represent a balance between the tendency to aggregate to increase predator safety and the tendency for large groups to fission as a consequence of increased food competition (Terborgh and Janson 1986). Thus, in order to optimize the consequences of group size variation, there should be selection for intermediate-sized or "optimal" group size in which individuals carry the lowest costs of group living and, therefore, ought to be healthiest and fittest (Chapman and Chapman 2000). Yet, intra-specific group size variation persists, but the proximate links between group size and health remain poorly understood.

One important link between sociality and health is the physiological stress response. Stressors are uncontrollable stimuli derived from extrinsic and intrinsic sources that trigger a reaction of the vertebrate hypothalamic-pituitary-adrenal (HPA) axis leading to the secretion of glucocorticoids (GCs) (Adkins-Regan 2005; Koolhaas et al. 2011). GCs are mainly responsible for the regulation of metabolic functions (Sapolsky et al. 2000; Beehner and Bergman 2017), in particular, mediating energy homeostasis during energetically demanding periods (Romero et al. 2009) and energetically costly behaviours, like locomotion (Dunn et al. 2013). When individuals enter a state of "stress", a rise of GC secretions initiates various behavioural and physiological changes to cope with the challenge (Busch and Hayward 2009), affecting individuals' immune functions and, therefore, ultimately also their health (Sapolsky et al. 2000; McEwen and Wingfield 2003; Busch and Hayward 2009).
Studies on group size effects on glucocorticoid output yielded a heterogeneous pattern across taxa, including primates, rodents, ungulates, and birds. While the majority of studies found a positive correlation between GC secretion and group size (Foley et al. 2001; Raouf et al. 2006; Dantzer et al. 2013; Dettmer et al. 2014), there were also studies reporting opposite findings (Michelena et al. 2012; Blondel et al. 2016), or no link (Snaith et al. 2008; Ebensperger et al. 2011). In principle, both, larger and smaller groups inflict energetic costs on individuals based on resource competition and predation risks (Chapman and Chapman 2000), which in turn may cause elevated GC concentrations. Hence, individuals in groups of intermediate or "optimal" sizes may face decreased energetic constraints and exhibit the lowest GC concentrations. However, only two studies, in ring-tailed lemurs (Lemur catta) and yellow baboons (Papio cynocephalus), found optimal group size effects in GCs (Pride 2005; Markham et al. 2015). The absence of such U-shaped correlations between group size and GCs in other prior investigations might be caused by biases towards studying larger groups (Markham et al. 2015). Hence, a lack of studies taking a species' full range of group sizes into account might be one explanation for the ambiguous GC patterns found across the literature. Nevertheless, group size-related benefits and costs for social animals are complex and numerous other factors are involved in shaping the relationship between group size and health.

First, the facilitated transmission of parasites and other pathogens constitutes one of the major costs of group living (Côté and Poulin 1995; Altizer et al. 2003; Kappeler et al. 2015; Müller-Klein et al. 2018). Several meta-analyses revealed that group size and parasite transmissions are generally positively correlated, but their relationship turns out to be rather variable and complex (Altizer et al. 2003; Rifkin et al. 2012; Patterson and Ruckstuhl 2013). This complexity is amplified by the interplay of parasites with GC secretion.

GCs can have suppressing effects on host immune function, which may lead to increased susceptibility to pathogens (Norbiato et al. 1997; Elenkov and Chrousos 1999; Turnbull and Rivier 1999). For instance, social stress in wild large vesper mice (Calomys callosus) caused not only elevated 
GC levels, but also an impaired immune response and increased blood infections with Trypanosoma cruzi (Santos et al. 2008). However, the relationship between GCs and parasites seems to be reciprocal as, for example, infections with Anguillicola novaezelandiae in European eels (Anguilla anguilla) positively affected individual cortisol levels (Dangel et al. 2014). Positive associations between GCs and parasite richness were also found in chimpanzees (Pan troglodytes), red colobus (Piliocolobus tephrosceles), and black howler monkeys (Alouatta pigra) (Chapman et al. 2006; Muehlenbein 2006; Martinez-Mota 2015). The multidirectional relationships between social parasite transmission and GCs emphasize the strong link between sociality and health.

Second, local variation in ecological factors, either as a function of variation in habitat quality or seasonal variation in resource availability, may impact group size effects on behavioural and physiological response variables. An implicit, but crucial assumption underlying most previous tests of the ecological constraints hypothesis is that the habitats of different groups do not differ in structure or quality. However, small-scale habitat features, like the distribution of food patches, can affect carrying capacities of home ranges and, therefore, influence population densities and group sizes (Marsh 1981; Iwamoto and Dunbar 1983; McLean et al. 2016; Strandburg-Peshkin et al. 2017). For example, mantled guerezas (Colobus guereza) formed larger groups when habitats provided more food trees (Dunbar 1987). Larger prides in lions (Panthera leo) were more likely to occupy territories of better quality, measured via six different landscape variables (Mosser and Packer 2009), and badger (Meles meles) group sizes increased with the quality of food patches (Kruuk and Parish 1982). Thus, significant local habitat heterogeneity might have important implications for species' group sizes.

Here, we provide a comprehensive test of the ecological constraints and optimal group size hypotheses by examining behavioural and physiological consequences of group size variation in a wild lemur population, Verreaux's sifakas (Propithecus verreauxi). These endemic Malagasy primates usually live in multimale-multifemale groups, even though groups can also comprise only single males and/or females. Their groups are comparably small, ranging from 2 to 12 individuals with a mean group size of 6, yet, like other primates (Majolo et al. 2008), they exhibit up to 5-fold variation in size (Jolly et al. 1982; Kappeler and Fichtel 2012; Sussman et al. 2012; Leimberger and Lewis 2015). Over a course of 2 years, we assessed ranging, activity, and dietary patterns of seven adjacent groups. Additionally, we measured individual levels of faecal glucocorticoid metabolites (fGCMs) and examined individual parasite richness. Intestinal parasite richness seems to be generally low in Verreaux's sifakas (Muehlenbein et al. 2003; Rasambainarivo et al. 2014; Springer and Kappeler 2016). However, here we apply a metabarcoding approach to assess infestations of intestinal helminths using next- generation sequencing of $18 \mathrm{~S}$ rRNA genes (Hadziavdic et al. 2014). Metabarcoding might be superior to previous methods, like microscopy, when assessing non-invasive parasite infestations as it allows for identification of a wide range of taxa of all life stages (i.e. eggs, larvae, and worms) and can recognize cryptic species (Aivelo and Medlar 2017).

To our knowledge, this is the first study in a mammal to simultaneously examine variation in ranging behaviour, daily activities, glucocorticoid metabolite levels, and parasitism as a function of group size while accounting for ecological stressors, namely food availability and average daily temperature differences. We expected to find one of two patterns in relation to group size: Either mean daily travel distances, home range sizes, parasite richness, and faecal GC metabolite concentrations should increase linearly with group size, according to predictions of the ecological constraints hypothesis, or these variables should follow a U-shaped pattern if groups of intermediate size are favoured, according to predictions of the optimal group size hypothesis. In case we should find heterogeneity among group habitats, we expect larger groups to inhabit areas with better quality, which should result in lower daily travel distances, foraging durations, and fGCMs for the respective groups.

\section{Materials and methods}

\section{Study site}

This study was conducted at the research station of the German Primate Center in Kirindy Forest, Western Madagascar (44 39' E, 20 03' S) from April 2016 to March 2018. Kirindy Forest is a protected dry deciduous forest and subject to pronounced seasonality, with a long, cool dry season (April to October) and a hot wet season (November to March) (Kappeler and Fichtel 2012).

\section{Study species}

We observed a total of 42 Verreaux's sifakas living in 7 adjacent groups ranging in size from 2 to 10 individuals, covering a broad range of group sizes found in this species with a maximum range of 1 to 12 individuals (Jolly et al. 1982; Sussman et al. 2012; Leimberger and Lewis 2015) (Table 1). Verreaux's sifakas are diurnal and arboreal primates with a mainly folivorous diet, but they exhibit pronounced seasonal dietary flexibility (Koch et al. 2017). They inhabit home ranges that remain stable over many years and partially overlap with those of neighbouring groups, but also include core areas of exclusive use (Benadi et al. 2008; Koch et al. 2016). All animals are habituated to human observers and individually marked with unique collars. 
Table 1 Summary of study groups and data collection

\begin{tabular}{lllllllllll}
\hline $\begin{array}{l}\text { Group } \\
\text { ID }\end{array}$ & $\begin{array}{l}\text { Group size } \\
\text { (mean) }\end{array}$ & $\begin{array}{l}\text { Adult } \\
+\end{array}$ & $\begin{array}{l}\text { Adult } \\
\text { ( }\end{array}$ & $\begin{array}{l}\text { Juveniles } \\
(<4 \text { years })\end{array}$ & $\begin{array}{l}\text { Infants } \\
(<9 \text { months })\end{array}$ & $\begin{array}{l}\text { Focal obs. } \\
(\mathrm{h})\end{array}$ & $\begin{array}{l}\text { GPS } \\
\text { days }\end{array}$ & $\begin{array}{l}\text { GPS } \\
\text { locations }\end{array}$ & $\begin{array}{l}\text { No. of samples } \\
\text { (fGCM) }\end{array}$ & $\begin{array}{l}\text { No. of samples } \\
\text { (parasites) }\end{array}$ \\
\hline F1 & $2-4(3)$ & 1 & 1 & $1-2$ & $0-1$ & 129 & 732 & 24070 & 160 & 41 \\
L & $3-4(4)$ & 1 & $1-2$ & $1-2$ & $0-1$ & 215 & 612 & 20084 & 307 & 59 \\
G & $4-6(5)$ & 2 & 1 & $1-2$ & $0-1$ & 233 & 729 & 23970 & 315 & 69 \\
M & $5-7(5)$ & $1-2 *$ & $2 *$ & $1-2 *$ & $0-1$ & 168 & 522 & 17179 & 203 & 42 \\
E & $5-7(6)$ & 1 & $2-3$ & $2-3$ & $0-1$ & 298 & 700 & 22998 & 378 & 82 \\
J & $5-7(7)$ & 2 & $1-2$ & $2-3$ & $0-1$ & 321 & 531 & 17437 & 347 & 91 \\
F & $8-10(10)$ & 2 & $1-3$ & $3-5$ & $0-2$ & 448 & 732 & 24010 & 619 \\
\hline
\end{tabular}

*As group M was a new group, marked and added to the study population in October 2016, we could not accurately assign individuals' ages. However, based on visual signs, including male chest stains, body size, behaviour, and the presence of an infant, we inferred the age of two males and one female to be above 4 years, while one female was estimated to be $3-4$ years of age

\section{Behavioural observations}

Focal animal sampling was carried out on all members of the 7 study groups, including adults and juveniles ( $>9$ months). Observations of $1 \mathrm{~h}$ per individual were conducted in an alternating order for $3 \mathrm{~h}$ in the morning and $3 \mathrm{~h}$ in the afternoon, resulting in a total of $1812 \mathrm{~h}$ of behavioural data. We continuously recorded all activities (social and non-social) as well as the identity of feeding plants and parts. As our study involved focal animal observations, it was not possible to record data blind.

\section{GPS data collection}

For assessing ranging patterns, one adult male per group was equipped with a GPS collar (e-obs, Grünwald, Germany) during annual captures (for details see Kappeler and Fichtel 2012). All collars were set to record GPS coordinates every $30 \mathrm{~min}$ between 04:00 and 20:00 h local time. As sifakas remain stationary on their sleeping tree during the night (Erkert and Kappeler 2004), we did not collect GPS locations between 20:00 and 04:00 $\mathrm{h}$ (Koch et al. 2016). On average, we recorded GPS data for 651 days with a mean of 21400 GPS locations per group (Table 1). For estimating home range sizes and core areas, we used monthly $95 \%$ and $50 \%$ fixed kernels using the adehabitatHR package (Calenge 2006) in R (R Version 3.4.4, R Core Team 2018). Daily travel distances (DTD) were calculated using the points-to-path plugin in Quantum GIS (QGIS Development Team 2018).

\section{Faecal sample collection and analyses}

During behavioural observations, fresh faecal samples, uncontaminated by urine, were collected within 3 min after defecation from the forest floor whenever they could be unequivocally assigned to an individual. Samples were collected weekly from all study animals except for dependent offspring.

\section{Hormone analyses}

Faecal samples for glucocorticoid metabolite (fGCM) analysis were collected in the morning between 07:00 and11:00 $\mathrm{h}(n=$ 2329) and placed in 15-ml polypropylene tubes (Sarstedt, Nümbrecht, Germany) containing $5 \mathrm{ml}$ of $80 \%$ ethanol. fGCM concentrations were determined upon subsequent extraction by using a validated enzyme immunoassay (EIA), measuring $5 ß$-reduced cortisol metabolites (Fichtel et al. 2007) (for details see Online Resource 1).

\section{Parasite analyses}

A total of 520 faecal samples were collected during four periods (April-May 2016/2017 and September-October 2016/2017). We collected up to four samples (Ø 3.9) per period from each study animal. Samples were stored in 2-ml polypropylene tubes containing $1 \mathrm{ml}$ RNAlater (Thermo Fisher Scientific, Waltham, MA, USA) at ambient temperature. After $24 \mathrm{~h}$, when RNAlater had completely soaked the faeces, samples were stored in a freezer at $-20{ }^{\circ} \mathrm{C}$ and remained frozen throughout shipping to Germany, where further analysis ensued.

Extraction of DNA, amplification, and sequencing of 18S rRNA genes We conducted DNA extraction with the PowerSoil DNA isolation kit (MoBio, Carlsbad, Canada). PCR reactions to generate eukaryotic 18S rRNA gene amplicons were performed in triplicates for each sample, then pooled in equimolar amounts and cleaned. Afterwards, we conducted dualindexed paired-end sequencing with the Illumina MiSeq platform and v3 chemistry (for details see Online Resource 1).

18S rRNA gene amplicon analyses Amplicon sequence variants (ASVs) were generated with VSEARCH version 2.9.1. We removed chimeric sequences with VSEARCH using UCHIME3 in de novo (-uchime3_denovo) and reference (- 
uchime ref) mode against the $\mathrm{PR}^{2}$ database (version 4.11.0) (Guillou et al. 2013). The following steps were conducted with the package ampvis2 (version 2.3.19) (Skytte et al. 2018) in R (version 3.4.4) (R Core Team 2018). We performed sample comparisons at the same surveying effort, using the lowest number of sequences by subsampling (2600 reads per sample). Additionally, we removed chloroplasts and extrinsic domains or unclassified ASVs from the data set (for details see Online Resource 1).

\section{Habitat quality}

Fixed area plots are a common sampling method in forest inventories (Scott 1998). For each group, 10 square plots ( $25 \times 25 \mathrm{~m})$ within the corresponding home range were randomly selected and all trees with a larger diameter than $5 \mathrm{~cm}$ were identified to the species level and the diameter at breast height (DBH) was measured (for details see Online Resource $1)$.

For estimating habitat quality, we examined feeding tree characteristics of each home range. We compared density, species richness, and sizes of a total of 6690 feeding trees belonging to 77 different species. These species were consumed during $67 \pm 4 \% \mathrm{SD}$ of time during groups' foraging bouts. Identification of feeding tree species is based on behavioural observations from June 2016 until March 2018 and comprises $539 \mathrm{~h}$ of observed feeding.

\section{Statistical analyses}

\section{GLMMs: group size effects on ranging patterns and fGCM concentrations}

We applied generalized linear mixed effect models (GLMMs) (Baayen et al. 2008) from the lme4 package (version: 1.1.21) (Bates et al. 2015) in R (version3.5.1) (R Core Team 2018), to test whether group size and group size-squared or group ID affect monthly averaged measures of DTDs, home range sizes, and individual fGCMs. We included study year (first or second), food availability, temperature differences ( $\Delta$ temperature), and sex (for fGCM models only) as fixed effects to control for ecological and social influences. Food availability was based on monitoring monthly phenology of 690 trees throughout the study period. We used a semi-quantitative method (Fournier 1974) in which the availability for each plant part (i.e. leaves, fruit, flowers) was scored, ranging from 0 (complete absence) to 4 (maximum abundance) (for details, see Koch et al. 2017). $\Delta$ temperatures describe the average monthly differences between daily minimum and maximum temperatures. We also examined interactions between group size and group size-squared with food availability and $\Delta$ temperatures in all respective models, to investigate group size effects on behavioural and physiological adaptations to seasonal changes. To keep type I error rates at the nominal level of 5\%, we included random slopes (Barr et al. 2013). $p$ values for individual effects were based on likelihood ratio tests comparing the full with the respective null models using the dropl function (Barr et al. 2013). If models resulted in significant effects of group ID, we conducted Tukey post hoc tests using the glht function of the package multcomp (version 1.4-10).

\section{GLMMs: group size effects on activity patterns}

To examine effects of group size or group ID on mean monthly foraging and resting rates, we calculated binomial models with beta error distribution structures and a logit link function using the glmmTMB package (version 0.2.3) (Brooks et al. 2017). Response variables (foraging and resting rates), predictors (group size or group ID, food availability, $\Delta$ temperatures), control variables (study year), random effects (group and animal ID), and random slopes (group size, food availability, $\Delta$ temperatures, and study year within group and animal ID) were included the same way as described above. We conducted full-null model comparisons and estimations of $p$ values as described above. Models resulting in significant effects of group ID were further analysed by comparing predicted marginal means using the lsmeans function of the package emmeans (version 1.3.3).

In all statistical analyses, our measure of group size comprised all present individuals, including adults (age $>4$ years), juveniles (age $<4$ years), and dependent infants (age $<$ 9 months) (Kappeler and Fichtel 2012). Yet, especially dependent infants may require and consume considerably less energy compared with juveniles and adults. As this could have important implications for how group size affects fGCM concentrations, activity, and ranging patterns, we re-ran all statistical analyses with a second measure of group size that excluded dependent infants (Online Resource 1, Tables S14S18). However, we did not find differences in model outcomes when using these two different measures of group size.

Moreover, we examined correlations between group sizes and core areas. As home range sizes were highly correlated with core areas (Pearson, $r=0.987 ; n=22 ; p<0.001$ ), we only considered home range sizes in our main analyses but added analyses on core areas to Online Resource 1 (Tables S19-S20, Fig. S3a, b).

\section{GLMMs: correlations among fGCMs, activity, and ranging patterns}

We additionally examined potential links between individual monthly fGCMs with ranging and activity patterns by applying two GLMMs as described above. We ln-transformed the response value (fGCMs) and included DTD or HR and foraging or resting rates as predictor variables. We included food 
availability as control variable. Monthly $\Delta$ temperatures were correlated with DTD and HR (Pearson: DTD/ $\Delta$ Temp, $r=-$ $0.82, n=22, p<0.001 ; \mathrm{HR} / \Delta \mathrm{Temp}, r-0.55, n=22$, $p<0.001$, Fig. S4c, d), which is why we excluded them from the model. Group and animal ID were utilized as random effects; and DTD or HR, foraging or resting rates, and food availability as random slopes within group and ID, respectively.

\section{ANOVA/Kruskal-Wallis: differences in habitat quality}

For comparing feeding tree characteristics (i.e. density, species diversity, and basal areas of all feeding trees) between the habitats, we conducted one-way ANOVAS or Kruskal-Wallis tests. Significant results were further analysed with Tukey tests using the glht function of the package multcomp (version 1.4-10) or with Dunn's pairwise post hoc tests (Bonferroni correction) using the package FSA (version 0.8.22), respectively.

See Online Resource 1 for more details on all statistical analyses.

\section{Results}

\section{Ranging patterns}

Verreaux's sifakas inhabited home ranges with a monthly average size of $15 \pm 7$ ha (mean \pm SD) and travelled on average $954 \pm 234 \mathrm{~m}$ per day. The model examining effects of group size, food availability, $\Delta$ temperatures, and study year on home range sizes was significant $\left(\chi^{2}=43.492, \mathrm{df}=9 p<0.001\right.$, $\left.R_{m / c}^{2}=0.23 / 0.79\right)$; however, group size was not correlated with home range size (Table S1, Fig. 1a). The second model, including group ID instead of group size as predictor variable, was also significant $\left(\chi^{2}=31.173, \mathrm{df}=6, p<0.001, R_{m / c}^{2}=\right.$ $0.70 / 0.72$ ) (Table S2, Fig. 2a), indicating that groups differed significantly in their home range sizes. Specifically, groups M and $\mathrm{L}$ had larger and group $\mathrm{J}$ had a smaller home range compared with the other groups (Table S5). Results of both models revealed that home range sizes were negatively correlated with food availability and $\Delta$ temperatures, which are smallest during the months of the wet season (Table S1, S2, Fig. S1a, b).

The model examining effects of group size, food availability, $\Delta$ temperatures, and study year on daily travel distances was significant $\left(\chi^{2}=65.410, \mathrm{df}=9 p<0.001, R_{m / c}^{2}=0.70 /\right.$ 0.82 ). There was an interaction effect with DTDs and $\Delta$ temperatures, i.e. during the dry season when $\Delta$ temperatures are increased, larger groups had shorter DTDs than smaller groups (test of the interaction between $\Delta$ temperatures and group size, $\chi^{2}=6.623 ; \mathrm{df}=1 ; p=0.010$ ) (Table S3). The model including group ID instead of group size as predictor variable was also significant $\left(\chi^{2}=76.405, \mathrm{df}=9, p<0.001, R_{m / c}^{2}=0.77 / 0.81\right)$ (Table S2, Fig. 2a) and groups differed significantly in their DTDs. Groups $\mathrm{M}$ and $\mathrm{L}$ covered longer distances than groups $\mathrm{J}$ and $\mathrm{F}$, while group $\mathrm{J}$ additionally had shorter DTDs compared with all groups except F1 (Table S5). In addition, DTDs were negatively correlated with food availability and $\Delta$ temperatures, which are smallest during the months of the wet season (Tables S3, S4, Fig. S1a, c).

\section{Daily activities}

Verreaux's sifakas spent on average $47 \pm 20 \%$ (mean \pm SD) of their time resting and $45 \pm 20 \%$ foraging. The model examining effects of group size, food availability, and $\Delta$ temperatures on monthly foraging rates was not significant $\left(\chi^{2}=7.379\right.$, $\mathrm{df}=5, p=0.194)$ (Table S6, Fig. 1c). In contrast, the model including group ID instead of group size as predictor variable was significant $\left(\chi^{2}=21.997, \mathrm{df}=8, p=0.005\right)$ (Table S7, Fig. $2 \mathrm{c}$ ), as group F1 and $\mathrm{G}$ had shorter foraging durations than several other groups (Table S10). Foraging rates were negatively correlated with $\Delta$ temperature, i.e. animals spent less time foraging during seasons with larger daily temperature changes, i.e. during the dry season (Table S7, Fig. S1a, d).

The model examining effects of group size, food availability, and $\Delta$ temperatures on monthly resting rates was not significant ( $\chi^{2}=6.195, \mathrm{df}=5, p=0.288$ ) (Table S8, Fig. 1d), while the model including group ID instead of group size as predictor variable was $\left(\chi^{2}=21.615, \mathrm{df}=8, p=0.006\right)$ (Table S9, Fig. 2d). Groups differed significantly in resting durations with groups $\mathrm{F} 1$ and $\mathrm{G}$ resting shorter than several other groups (Table S10). Resting rates were positively correlated with $\Delta$ temperature, i.e. animals spent more time resting during seasons with larger daily temperatures changes, i.e. during the dry season (Tables S9, Fig. S1a, e).

\section{Faecal glucocorticoid metabolites}

On average, individuals had mean monthly fGCM concentrations of $0.326 \pm 0.181 \mu \mathrm{g} / \mathrm{g}$ (mean $\pm \mathrm{SD}$ ). Males had generally higher average fGCM concentrations $(0.360 \pm 0.174 \mu \mathrm{g} / \mathrm{g})$ than females $(0.276 \pm 0.181 \mu \mathrm{g} / \mathrm{g})$ (Table S11, S12). The model examining effects of group size, food availability, $\Delta$ temperatures, study year, and sex on monthly fGCM concentrations was significant $\left(\chi^{2}=51.406, \mathrm{df}=10, p<0.001, R_{m / c}^{2}=\right.$ $0.20 / 0.36$ ); however, group size was not correlated with FGCM concentrations (Table S11, Fig. 1e). The model including group ID instead of group size as predictor variable was also significant $\left(\chi^{2}=55.800, \mathrm{df}=10, p<0.001, R_{m / c}^{2}=0.21 /\right.$ 0.32 ), but groups did not differ in fGCM concentrations (Table S12, Fig. 2e). Sifaka's fGCM concentrations were positively correlated with food availability and $\Delta$ temperatures, i.e. which are largest during the dry season (Table S11, S12, Fig. S1, f, g). 
Fig. 1 Group size versus different behavioural and physiological variables. In graphs $(\mathbf{a}-\mathbf{e})$, boxplots comprise data on mean monthly rates per group (for home range size and daily travel distances) or individual (foraging, resting, fGCMs) and indicate median, upper, and lower quartiles. Whiskers indicate \pm 1.5 interquartile ranges and small circles beyond whiskers indicate outliers. In graph (f), data points represent analysed samples and horizontal lines indicate medians

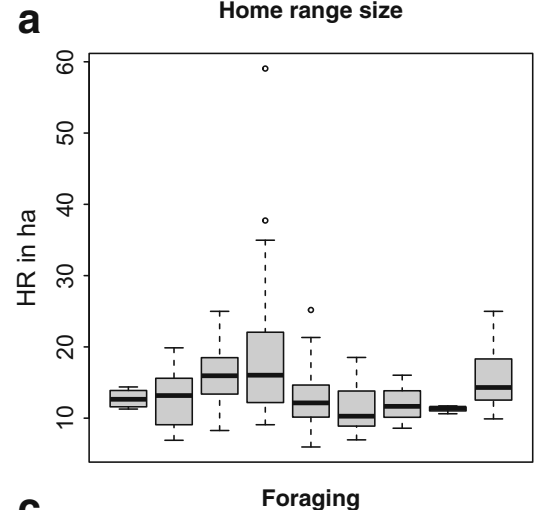

b

Daily travel distance

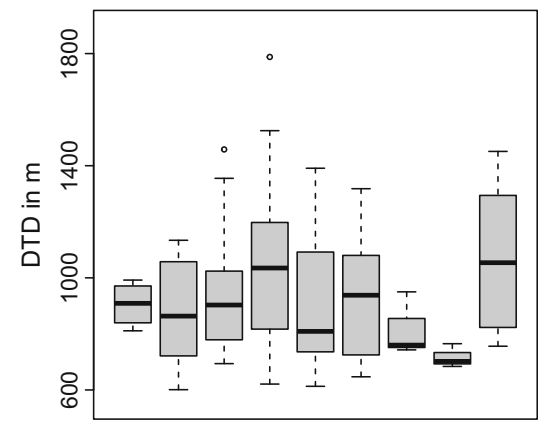

d

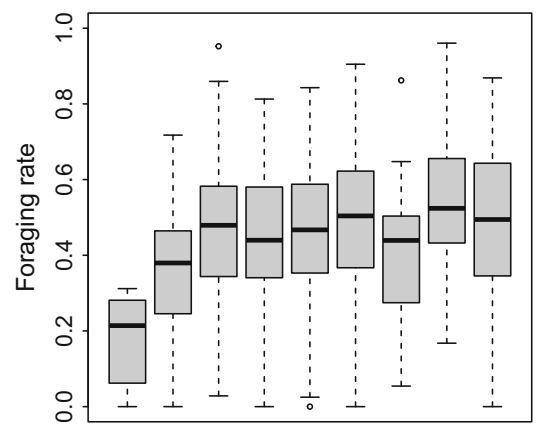

e

Faecal glucocorticoid metabolites

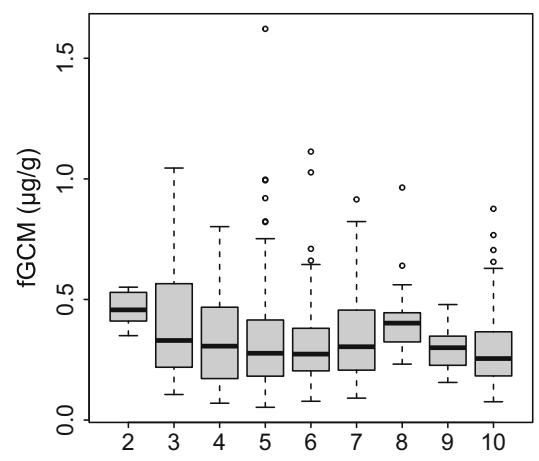

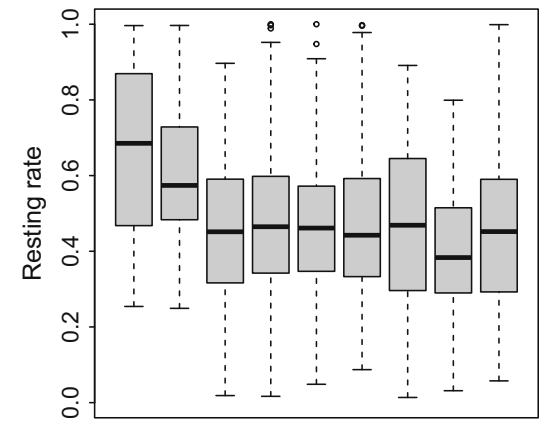

f

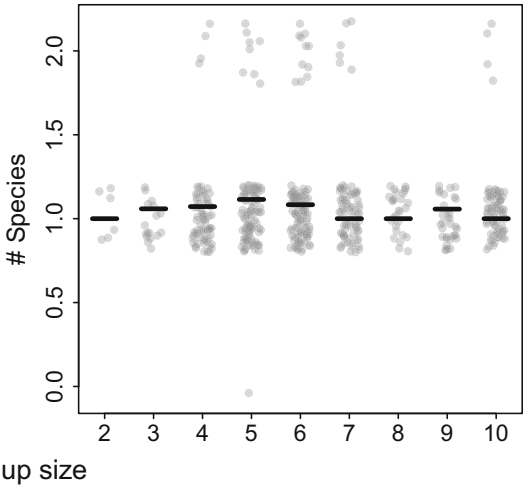

\section{Correlations among fGCMs and activity and ranging patterns}

The models on links between individual monthly fGCMs with DTDs and foraging rates or HRs and resting rates were both highly significant (DTD + foraging, $\chi^{2}=20.210, \mathrm{df}=2$, $p<0.001, R_{m / c}^{2}=0.13 / 0.29 ; \mathrm{HR}+$ resting $\chi^{2}=18.981, \mathrm{df}=$ $\left.2, p<0.001, R_{m / c}^{2}=0.14 / 0.31\right)$. More precisely, DTDs and HRs were negatively correlated with fGCMs, while time spent resting was positively correlated. Foraging rates were not correlated with fGCMs (Tables S22, S23, Fig. S5).

\section{Parasite richness}

After subsampling, 33 of 520 samples were removed due to low read numbers. The remaining 487 faecal samples contained 6587 eukaryotic ASVs and 24.764.794 reads. A total of 2947 of all ASVs could be taxonomically assigned and belonged to nine phyla: Opisthokonta (1288), Archaeplastida (730), Alveolata (628), Rhizaria (194), Amoebozoa (116), Stramenopiles (36), Hacrobia (10), Apusozoa (3), and Excavata (2). In terms of nematodes, 3 different families known to contain parasitic species were present in the samples: Trichostrongylidae, Onchocercidae, and Oxyuridae (Fig. S2).

All individuals repeatedly tested PCR-positive for infestation with nematodes of the family Trichostrongylidae (486/ 487 samples). Additionally, in 33 samples from 26 individuals representing all study groups, nematodes of the family Oxyuridae appeared at least once $(n=21)$ and up to three times $(n=5)$ during both dry seasons. Another 32 samples of 18 individuals were positive for filarial nematodes of the family Onchoceridae. However, parasites of this family are 
Fig. 2 Group ID versus different behavioural and physiological variables. Groups are ordered by mean group size, with the smallest group being depicted on the left. Highlighted graphs indicate significant differences among groups. Boxplots comprise data on mean monthly values per group (for home range size and daily travel distances) or individual (foraging, resting, fGCMs) and indicate median, upper, and lower quartiles. Whiskers indicate \pm 1.5 interquartile ranges and small circles beyond whiskers indicate outliers. In graph (f), data points represent analysed samples and horizontal lines indicate medians. Different letters indicate significant differences in means, i.e. groups sharing at least one letter do not differ
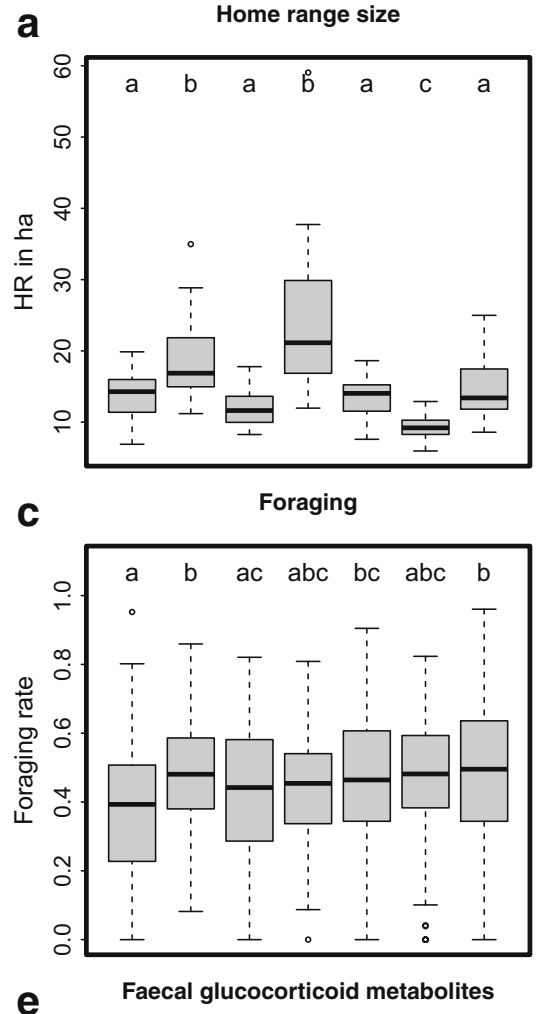

b

Daily travel distance

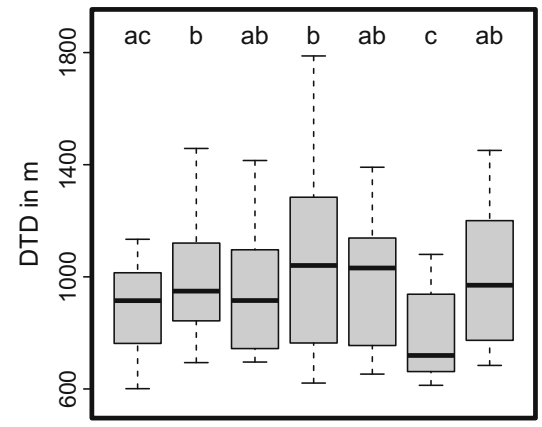

d
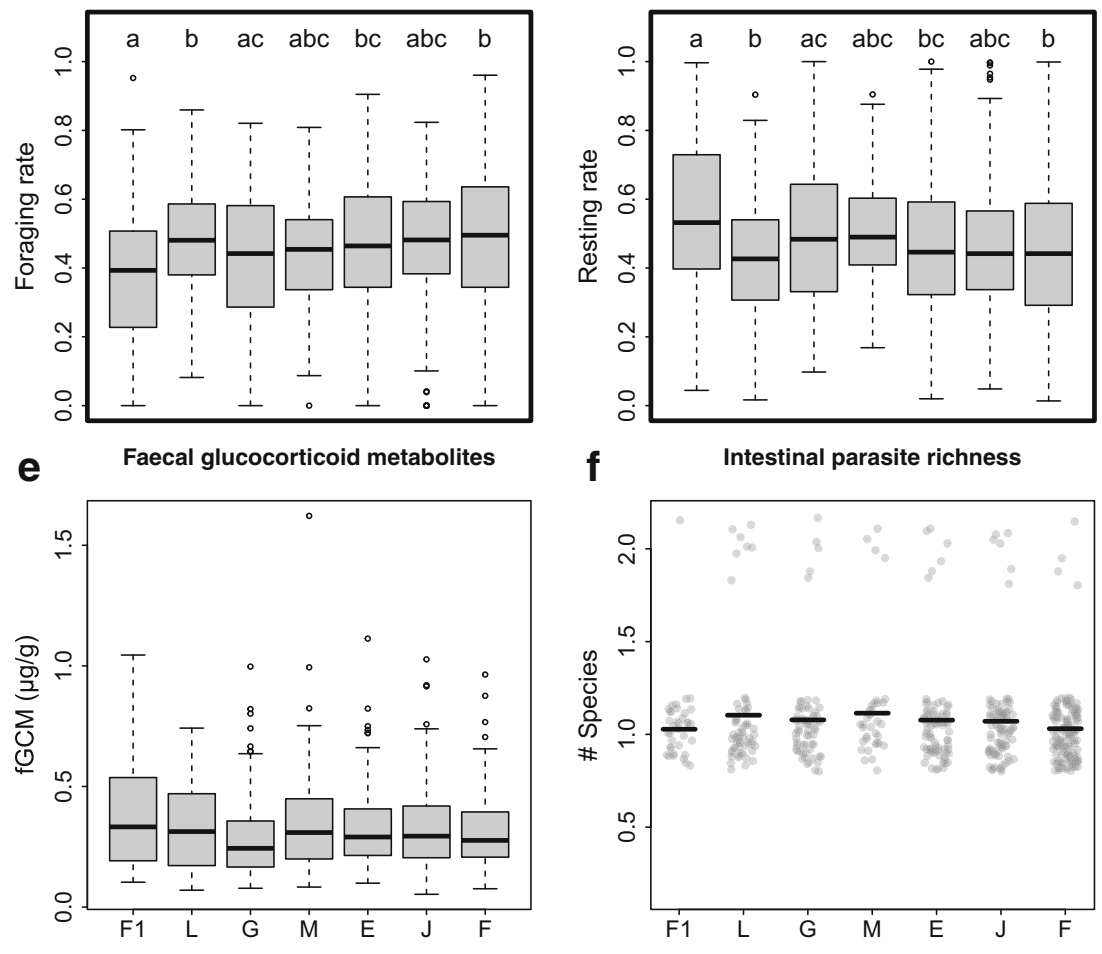

f

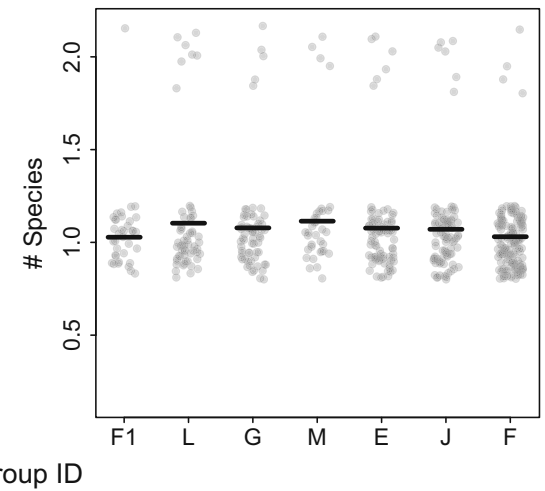

vector-borne and usually occur in body fluids or particular tissues. Finding their DNA in faecal samples might be a result of small perforations of sifakas' intestines, leading to small amounts of blood containing the parasites entering the colon, or accidental ingestion of vectors during feeding or oral grooming. Prevalence of Onchocercidae was, therefore, not considered in our estimation of gastro-intestinal parasite richness. Hence, with all animals carrying Trichostrongylidae, and Oxyuridae only occurring in a few samples, intestinal parasite richness across all groups averages 1 and is not affected by group size.

\section{Habitat quality}

Groups' habitats differed in food tree density (Kruskal-Wallis, $\left.\chi^{2}=25.723 ; \mathrm{df}=6 ; p<0.001\right)$ and species richness (ANOVA,
$\left.F_{6,59}=2.34 ; p=0.043\right)$; however, these differences were not related to group size (Pearson: food tree density, $r=-0.287$, $n=7, p=0.533$; species richness, $r=-0.080, n=7, p=865$ ). For tree densities, post hoc Dunn's test revealed that the habitat of group $\mathrm{M}$ harboured significantly more trees than the habitats of groups $\mathrm{G}$, J, and L. Food tree richness only differed between habitats of groups $\mathrm{M}$ and $\mathrm{J}$, with $\mathrm{M}$ having a higher richness (Table S15; Fig. 3). We found no differences in food tree sizes among the different habitats (Kruskal-Wallis: basal area, $\left.\chi^{2}=4.642 ; \mathrm{df}=6 ; p=0.590\right)$ (Fig. 3).

\section{Discussion}

In this study, we examined behavioural and physiological consequences of group size variation in wild Verreaux's sifakas. 
a

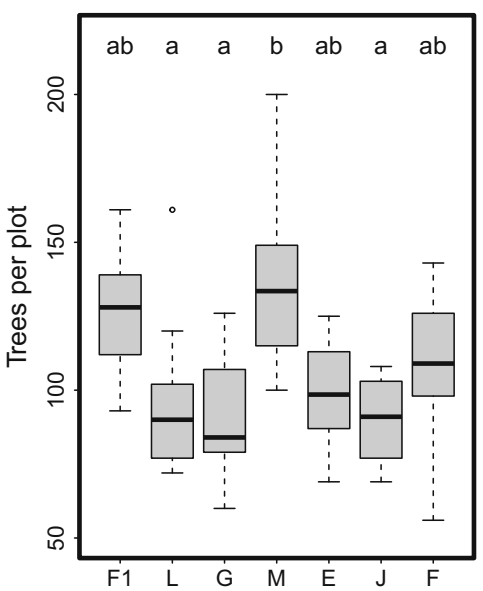

b Food tree species richness

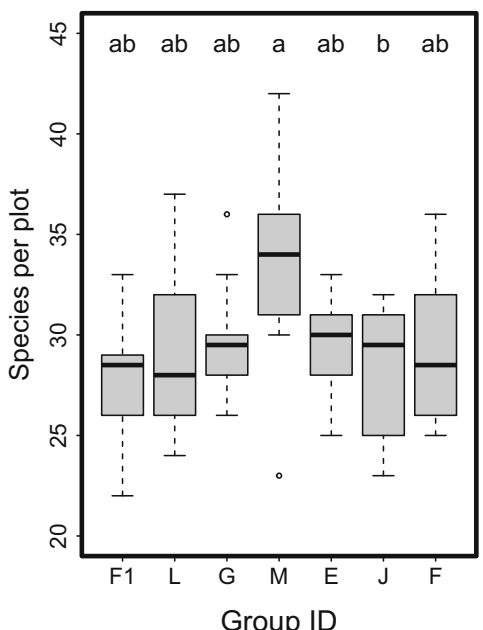

C

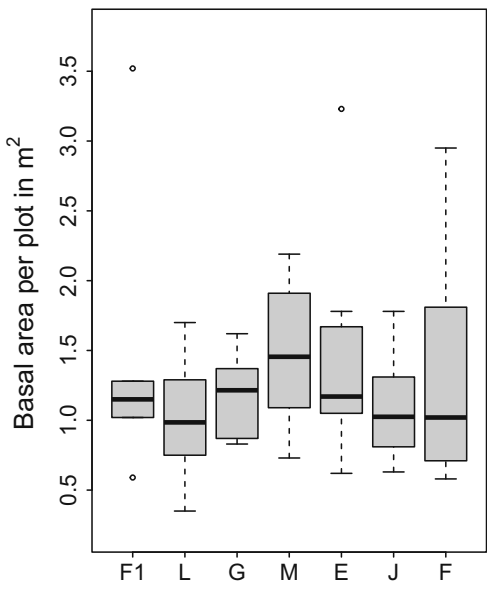

Fig. 3 Habitat characteristics of the home ranges of seven sifaka groups. Groups are ordered by mean group size, with the smallest group being depicted on the left. Boxplots show density, richness, and basal areas of the feeding trees of 10 plots per group ( 6 plots for group F1). Boxplots indicate median, upper, and lower quartiles. Whiskers indicate \pm 1.5 interquartile ranges and small circles beyond whiskers indicate outliers. Highlighted graphs indicate significant differences among groups. Different letters indicate significant differences in means, i.e. groups sharing at least one letter do not differ

groups indicate no need for compensation of energetic disadvantages resulting from variation in group size. Considering that Verreaux's sifakas form relatively small groups, food competition might also not be strong enough to significantly affect individual energy budgets.

We found, however, variation in habitat quality and varying ranging patterns and daily activity among some groups. Intergroup differences in ranging behaviour have been linked to differences in local food distribution before (Caraco 1979; Altmann and Muruthi 1988; Isbell 1991; Bronikowski and Altmann 1996), and studies in baboons and three Neotropical primates revealed changes in movement patterns as a response to different habitat features (McLean et al. 2016; Strandburg-Peshkin et al. 2017). Yet, variation in habitat quality does not seem to explain different ranging and activity patterns among our study groups. For example, group M's habitat had a higher density and richness of food trees compared with group J. Hence, group M's habitat might be of better quality, which should result in reduced energetic costs due to decreased travelling and foraging efforts. Yet, group $\mathrm{M}$ had higher daily travel distances than group J, while there was no difference in foraging or resting rates between these two groups. It is possible that this intergroup variation could be related to locations of key food patches within the groups' habitats. While food abundance within the habitats seems sufficient to provide all groups, suboptimal distributions of preferred food trees might require some groups to travel farther then others within their home ranges. However, we did not determine exact locations of food resources and can therefore not evaluate this hypothesis. 


\section{Group size, seasonality, and fGCMs}

fGCMs are often used as indicators of energetic trade-offs individuals face when living in groups (Markham and Gesquiere 2017). Life in smaller and larger groups is supposed to inflict higher energetic costs on individuals (Chapman and Chapman 2000). However, average concentrations of fGCMs did not differ with variation in group size in our study population. As none of the other measures (i.e. parasitism, ranging, and activity patterns), which might induce such energetic trade-offs, varied with group size either, these results are conclusive. Since sifaka groups did vary in ranging and activity patterns without varying in fGCM concentrations, they appear to be able to apply behavioural adaptations to environmental and/or social challenges without suffering major energetic disadvantages independent of their group size.

Notably, individual fGCM concentrations were strongly affected by seasonal variation in temperature differences and food availability. Ecological and behavioural season, however, are tightly interwined in sifakas, as the birth season falls into the middle of the cool dry season and mating season takes place around the peak of the hot wet season (Kappeler and Fichtel 2012). Thus, female reproductive state and increased energetic demands for males during the mating season (Fichtel et al. 2007) represent likely confounding factors for glucocorticoid outputs. Additionally, climatic season and unpredictability are more pronounced in Madagascar than in many other tropical regions (Dewar and Richard 2007). In Kirindy Forest, food availability is closely linked to seasonality and known to affect sifakas' diet, daily activities, and ranging patterns (Norscia et al. 2006; Koch et al. 2017). In sum, as individual variation in GC production is determined by various metabolic, social, and environmental stressors, it can be difficult to identify effects of single variables (Huber et al. 2003; Foerster and Monfort 2010).

Interestingly, ranging patterns where negatively correlated with fGCM concentrations. This finding stands in contrast to the assumption that energetically demanding behaviours, like periods of increased locomotion, are reflected in increased GC secretions (Sapolsky et al. 2000; Beehner and Bergman 2017). Both measures were correlated with seasonal changes; yet, while ranging patterns decreased with food availability and increased with temperature differences, fGCM concentrations showed opposite correlations. This suggests that Verreaux's sifakas reduce ranging activities to cope with the increased energetic requirements of thermoregulation during the middle of the dry season, when minimum and maximum temperatures differ most strongly and can fall to $3{ }^{\circ} \mathrm{C}$ (Kappeler and Fichtel 2012). These energetic requirements appear to result in increased fGCM concentrations and are potentially fortified by the low food availability during this season. The negative correlation between ranging patterns and fGCMs highlights the complexity of fGCMs patterns and emphasizes the potential of confounding factors in studies of fGCMs. In addition, we could show, similar to numerous studies in other vertebrates (Romero 2002), that sifaka's monthly fGCMs changed simultaneously with behavioural and seasonal patterns.

\section{Group size and parasites}

We detected three families of nematodes, two (Trichostrongylidae and Onchocercidae) of which have been previously reported in the same study population (Springer and Kappeler 2016) and one (Oxyuridae) which has been previously reported in the same species but different population (Rasambainarivo et al. 2014). Contrary to our predictions, there was no correlation between group size and intestinal parasite richness. Larger groups are expected to harbour more different parasites due to more opportunities for direct or indirect transmission via social contact or shared environments (Nunn et al. 2003). Although parasites are socially transmitted in Verreaux's sifakas (Springer and Kappeler 2016), our study animals exhibited low parasite richness, which was prevalent in almost all individuals, making it difficult to determine the mode of transmission. Hence, the costs of sociality in terms of parasite spread could not be estimated in this species.

Seasonality affected the prevalence of Oxyuridae, as they were only detected in samples collected during the dry season, confirming earlier results (Springer and Kappeler 2016). The dry season in Kirindy Forest represents an energetically demanding period as reflected in sifakas reduced ranging patterns and increased fGCM concentrations. It is likely that animals are more susceptible to parasites due to impaired and energetically costly immune functions during this time (Sheldon and Verhulst 1996). Given the complexity of hostparasite interactions, the effects of host group size on parasite richness might depend on various other aspects of sociality, species-specific behaviours, and environmental factors that can affect contact and, therefore, transmission rates (Patterson and Ruckstuhl 2013).

So far, only a handful of studies have used metabarcoding to non-invasively assess intestinal parasites in animals (Wimmer et al. 2004; Tanaka et al. 2014; Avramenko et al. 2015; Srivathsan et al. 2016). This novel approach can facilitate differentiation among closely related species within the same sample and, nowadays, constitutes a faster, cheaper, and more precise method in comparison with many traditional analyses (Aivelo et al. 2018). Accordingly, this approach allowed us to detect a parasite (Oxyuridae) that has not been found in this population before by studies using conventional microscopic methods (Springer and Kappeler 2016).

\section{An optimal group size?}

Major costs and benefits of group living are generally linked to predators, pathogens, and resource competition, which 
ultimately affect individual well-being. Variation in group size reflects adaptations to local ecological conditions and represents one strategy of balancing the various advantages and disadvantages of group living. However, if, for various reasons, groups exceed their upper or lower "optimal" limits in size, the costs of sociality are expected to outweigh the benefits, and groups should, therefore, split or fusion with others (Majolo and Huang 2018).

In Verreaux's sifakas, even though groups are small with a population-wide average of 6 individuals (Jolly et al. 1982; Sussman et al. 2012; Leimberger and Lewis 2015), they varied up to 5-fold in size in our study. Yet, we did not detect substantial group size-related behavioural or physiological differences between individuals. Maybe not all assumed major costs and benefits of group living apply to this species. For example, predation risk does not seem to strongly impact Verreaux's sifakas' group sizes as another population in Berenty Reserve, where terrestrial predators are absent (Jolly 2012), exhibits a similar mean and variance in group size (Jolly et al. 1982; Norscia and Palagi 2008; Kappeler and Fichtel 2012). Additionally, due to their small groups and mainly folivorous diet, competition over food should play a less important role in shaping group size. Like other folivorous taxa, sifakas are expected to form much larger groups than they actually do. The leading hypothesis for this "folivore paradox" invokes social (i.e. male takeovers and infanticide risks) instead of ecological factors as the main constraint on group size (Treves and Chapman 1996; Steenbeek and van Schaik 2001). Thus, in Verreaux's sifakas, and perhaps other folivorous species in general, group sizes appear to remain below the upper, ecologically "optimal" limits because social rather than ecological factors define these limits. This notion may explain why we did not detect any of the group size-related costs, proposed by the ecological constraints and optimal group size hypotheses. Our findings on between-group variation in daily activities and ranging patterns suggest that differences in microhabitat features shape fine-grained behavioural adaptations. However, these behavioural differences were not reflected in groups' fGCM concentrations and parasites richness, indicating that all groups, independent of their size, can compensate potential habitatrelated challenges without causing health-related costs. An optimal group size might, therefore, not exist in Verrreaux's sifakas.

\section{Conclusions}

This study contributes to the understanding of the relationship between group size, health, and ecology in vertebrates. We show that group size on its own might be insufficient to explain links between sociality and health, probably due to the complex and multifaceted nature of this relationship. We also demonstrate strong impacts of environmental factors on socioecological traits that might obscure patterns in the socialityhealth nexus. Altogether, our results do not support predictions of the ecological constraints hypothesis and the optimal group size hypothesis as they may only hold true for species limited in group size by ecological factors - a condition that may not apply to the majority of folivorous mammals.

Acknowledgments We are grateful to the Malagasy Ministère de l'Environnement, the Departement de Biologie Ecologie et Conservation Animale de l'Université d'Antananarivo, and the Centre National de Formation, d'Etudes et de Recherche en Environnementet Foresterie de Morondava for supporting our research in Kirindy Forest. We also thank Mamy Razafindrasamba and Tiana Andrianjanahary for their support in data collection and Dr. Roger Mundry for statistical advice. We also thank two anonymous reviewers for their valuable and constructive comments. The study profited greatly from discussions with members of the DFG research unit "Sociality and Health in Primates" (FOR 2136).

Statement of authorship PMK, CF, and KR designed the study. KR and FK performed data collection. MH supervised the hormone analyses. KR conducted the lab work and analysed the data. DS helped with the analysis of the parasite data. KR drafted the manuscript and all authors contributed to writing and revising of the manuscript.

Funding information This research was financially supported by the Deutsche Forschungsgemeinschaft (DFG), grant number Ka 1082/29-2, awarded to PMK.

Data availability statement Sequences have been deposited in the NCBI GenBank under the project number PRJNA527362. All other data generated or analysed during this study are included in this published article as supplementary information files (Online Resource 2).

\section{Compliance with ethical standards}

Conflict of interest The authors declare that they have no conflict of interest.

Ethical approval All applicable international, national, and/or institutional guidelines for the care and use of animals were followed. Statement of ethical approval, approval of research protocols, and capture procedures was approved by a committee of the Ministry for the Environment, Water and Forests of Madagascar (MINEEF) (Permit numbers: 50/16/MEEMF/SG/DGF/DAPT/SCBT.Re, 234/16/MEEF/SG/ DGF/DSAP/SCB.Re, 72/17/MEEF/SG/DGF/DSAP/SCB.Re, 250/17/ $\mathrm{MEEF} / \mathrm{SG} / \mathrm{DGF} / \mathrm{DSAP} / \mathrm{SCB} . \mathrm{Re})$. All procedures performed in studies involving animals were in accordance with the ethical standards of the institution or practice at which the studies were conducted. This article does not contain any studies with human participants performed by any of the authors.

Open Access This article is distributed under the terms of the Creative Commons Attribution 4.0 International License (http:// creativecommons.org/licenses/by/4.0/), which permits unrestricted use, distribution, and reproduction in any medium, provided you give appropriate credit to the original author(s) and the source, provide a link to the Creative Commons license, and indicate if changes were made. 


\section{References}

Adkins-Regan E (2005) Hormones and animal social behavior. Princeton University Press, Princeton

Aivelo T, Medlar A (2017) Opportunities and challenges in metabarcoding approaches for helminth community identification in wild mammals. Parasitology 145:608-621. https://doi.org/10. 1017/S0031182017000610

Aivelo T, Medlar A, Löytynoja A, Laakonen J, Jernvall J (2018) Metabarcoding gastrointestinal nematodes in sympatric endemic and nonendemic species in Ranomafana National Park, Madagascar. Int J Primatol 39:49-64. https://doi.org/10.1007/ s10764-017-0010-x

Altizer S, Nunn CL, Thrall PH, Gittleman JL, Antonovics J, Cunningham AA, Dobson AP, Ezenwa V, Jones KE, Pedersen AB, Poss M, Pulliam JRC (2003) Social organization and parasite risk in mammals: integrating theory and empirical studies. Annu Rev Ecol Evol Syst 34:517-547. https://doi.org/10.1146/annurev.ecolsys.34. 030102.151725

Altmann J, Muruthi P (1988) Differences in daily life between semiprovisioned and wild-feeding baboons. Am J Primatol 15: 213-221. https://doi.org/10.1002/ajp.1350150304

Avramenko RW, Redman EM, Lewis R, Yazwinski TA, Wasmuth JD, Gilleard JS (2015) Exploring the gastrointestinal "Nemabiome": deep amplicon sequencing to quantify the species composition of parasitic nematode communities. PLoS One 10:e0143559. https:// doi.org/10.1371/journal.pone.0143559

Baayen RH, Davidson DJ, Bates DM (2008) Mixed-effects modeling with crossed random effects for subjects and items. J Mem Lang 59:390-412. https://doi.org/10.1016/j.jml.2007.12.005

Barr DJ, Levy R, Scheepers C, Tily HJ (2013) Random effects structure for confirmatory hypothesis testing: keep it maximal. J Mem Lang 68:255-278. https://doi.org/10.1016/j.jml.2012.11.001

Bates D, Maechler M, Bolker B, Walker S (2015) Fitting linear mixedeffects models using lme4. J Stat Softw 67:1-48

Beehner JC, Bergman TJ (2017) The next step for stress research in primates: to identify relationships between glucocorticoid secretion and fitness. Horm Behav 91:68-83. https://doi.org/10.1016/j.yhbeh. 2017.03.003

Benadi G, Fichtel C, Kappeler P (2008) Intergroup relations and home range use in Verreaux's sifaka (Propithecus verreauxi). Am J Primatol 70:956-965. https://doi.org/10.1002/ajp.20588

Blondel DV, Wallace GN, Calderone S, Gorinshteyn M, St. Mary CM, Phelps SM (2016) Effects of population density on corticosterone levels of prairie voles in the field. Gen Comp Endocrinol 225:13-22. https://doi.org/10.1016/j.ygcen.2015.09.002

Borries C, Larney E, Lu A, Ossi K, Koenig A (2008) Costs of group size: lower developmental and reproductive rates in larger groups of leaf monkeys. Behav Ecol 19:1186-1191. https://doi.org/10.1093/ beheco/arn088

Bronikowski AM, Altmann J (1996) Foraging in a variable environment: weather patterns and the behavioral ecology of baboons. Behav Ecol Sociobiol 39:11-25. https://doi.org/10.1007/s002650050262

Brooks ME, Kristensen K, van Benthem KJ, Magnusson A, Berg CW, Nielsen A, Skaug HJ, Machler M, Bolker BM (2017) glmmTMB balances speed and flexibility among packages for zero-inflated generalized linear mixed modeling. R J 9:378-400. https://doi.org/ 10.3929/ethz-b-000240890

Busch DS, Hayward LS (2009) Stress in a conservation context: a discussion of glucocorticoid actions and how levels change with conservation-relevant variables. Biol Conserv 142:2844-2853. https://doi.org/10.1016/j.biocon.2009.08.013

Calenge C (2006) The package "adehabitat" for the R software: a tool for the analysis of space and habitat use by animals. Ecol Model 197: 516-519. https://doi.org/10.1016/j.ecolmodel.2006.03.017
Caraco T (1979) Time budgeting and group size: a theory. Ecology 60: 611-617. https://doi.org/10.2307/1936081

Chapman CA, Chapman LJ (2000) Determinants of group size in primates: the importance of travel costs. In: Boinski S, Garber PA (eds) On the move: how and why animals travel in groups. University of Chicago Press, Chicago, pp 24-42

Chapman CA, Wasserman MD, Gillespie TR, Speirs ML, Lawes MJ, Saj TL, Ziegler TE (2006) Do food availability, parasitism, and stress have synergistic effects on red colobus populations living in forest fragments? Am J Phys Anthropol 131:525-534. https://doi.org/10. 1002/ajpa.20477

Côté IM, Poulin R (1995) Parasitism and group size in social animals: a meta-analysis. Behav Ecol 6:159-165. https://doi.org/10.1093/ beheco/6.2.159

Dangel KC, Keppel M, Tabujew K, Sures B (2014) Effects of Anguillicola novaezelandiae on the levels of cortisol and hsp70 in the European eel. Parasitol Res 113:3817-3822. https://doi.org/10. 1007/s00436-014-4049-8

Dantzer B, Newman AEM, Boonstra R, Palme R, Boutin S, Humphries MM, McAdam AG (2013) Density triggers maternal hormones that increase adaptive offspring growth in a wild mammal. Science 340: 1215-1217. https://doi.org/10.1126/science. 1235765

Dettmer AM, Novak MA, Meyer JS, Suomi SJ (2014) Population density-dependent hair cortisol concentrations in rhesus monkeys (Macaca mulatta). Psychoneuroendocrinology 42:59-67. https:// doi.org/10.1016/j.psyneuen.2014.01.002

Dewar RE, Richard AF (2007) Evolution in the hypervariable environment of Madagascar. Proc Natl Acad Sci USA 104:13723-13727. https://doi.org/10.1073/pnas.0704346104

Dunbar RIM (1987) Habitat quality, population dynamics, and group composition in Colobus Monkeys (Colobus guereza). Int $\mathrm{J}$ Primatol 8:299-329. https://doi.org/10.1007/BF02737386

Dunn JC, Cristóbal-Azkarate J, Schulte-Herbrüggen B, Chavira R, Veà JJ (2013) Travel time predicts fecal glucocorticoid levels in freeranging howlers (Alouatta palliata). Int J Primatol 34:246-259. https://doi.org/10.1007/s10764-013-9657-0

Ebensperger LA, Ramírez-Estrada J, León C, Castro RA, Ortitz Tolhuysen L, Quirici V, Burger JR, Soto-Gamboa M, Hayes LD (2011) Sociality, glucocorticoids and direct fitness in the communally rearing rodent, Octodon degus. Horm Behav 60:346-352. https:// doi.org/10.1016/j.yhbeh.2011.07.002

Elenkov IJ, Chrousos GP (1999) Stress hormones, Th1/Th2 patterns, pro/ anti-inflammatory cytokines and susceptibility to disease. Trends Endocrinol Metab 10:359-368. https://doi.org/10.1016/S10432760(99)00188-5

Erkert HG, Kappeler PM (2004) Arrived in the light: diel and seasonal activity patterns in wild Verreaux's sifakas (Propithecus v. verreauxi; Primates: Indriidae). Behav Ecol Sociobiol 57:174-186. https://doi. org/10.1007/s00265-004-0845-y

Ezenwa VO, Ghai RR, McKay AF, Williams AE (2016) Group living and pathogen infection revisited. Curr Opin Behav Sci 12:66-72. https:// doi.org/10.1016/j.cobeha.2016.09.006

Fichtel C, Kraus C, Ganswindt A, Heistermann M (2007) Influence of reproductive season and rank on fecal glucocorticoid levels in freeranging male Verreaux's sifakas (Propithecus verreauxi). Horm Behav 51:640-648. https://doi.org/10.1016/j.yhbeh.2007.03.005

Foerster S, Monfort SL (2010) Fecal glucocorticoids as indicators of metabolic stress in female Sykes' monkeys (Cercopithecus mitis albogularis). Horm Behav 58:685-697. https://doi.org/10.1016/j. yhbeh.2010.06.002

Foley CAH, Papageorge S, Wasser SK (2001) Noninvasive stress and reproductive measures of social and ecological pressures in freeranging African elephants. Conserv Biol 15:1134-1142. https:// doi.org/10.1046/j.1523-1739.2001.0150041134.x

Fournier LA (1974) Un método cuantitativo para la medición de características fenológicas en árboles. Turrialba 24:422-423 
Guillou L, Bachar D, Audic S, Bass D, Berney C, Bittner L, Boutte C, Burgaud G, de Vargas C, Decelle J, del Campo J, Dolan JR, Dunthorn M, Edvardsen B, Holzmann M, Kooistra WHCF, Lara E, le Bescot N, Logares R, Mahé F, Massana R, Montresor M, Morard R, Not F, Pawlowski J, Probert I, Sauvadet AL, Siano R, Stoeck T, Vaulot D, Zimmermann P, Christen R (2013) The Protist Ribosomal Reference database (PR2): a catalog of unicellular eukaryote small sub-unit rRNA sequences with curated taxonomy. Nucleic Acids Res 41:D597-D604. https://doi.org/10.1093/nar/ gks1160

Hadziavdic K, Lekang K, Lanzen A, Jonassen I, Thompson EM, Troedsso C (2014) Characterization of the 18S rRNA gene for designing universal eukaryote specific primers. PLoS One 9:e87624. https://doi.org/10.1371/journal.pone.0087624

Huber S, Palme R, Arnold W (2003) Effects of season, sex, and sample collection on concentrations of fecal cortisol metabolites in red deer (Cervus elaphus). Gen Comp Endocrinol 130:48-54. https://doi. org/10.1016/S0016-6480(02)00535-X

Isbell LA (1991) Contest and scramble competition: patterns of female aggression and ranging behavior among primates. Behav Ecol 2: 143-155. https://doi.org/10.1093/beheco/2.2.143

Iwamoto T, Dunbar RIM (1983) Thermoregulation, habitat quality and the behavioural ecology of gelada baboons. J Anim Ecol 52:357366. https://doi.org/10.2307/4559

Janson CH, Goldsmith ML (1995) Predicting group size in primates: foraging costs and predation risks. Behav Ecol 6:326-336. https:// doi.org/10.1093/beheco/6.3.326

Jolly A (2012) Berenty Reserve, Madagascar: a long time in a small space. In: Kappeler PM, Watts DP (eds) Long-term field studies of primates. Springer, Berlin, pp 21-44

Jolly A, Gustafson H, Oliver WLR, O’Connor SM (1982) Propithecus verreauxi population and ranging at Berenty, Madagascar, 1975 and 1980. Folia Primatol 39:124-144. https://doi.org/10.1159/ 000156071

Kappeler PM, Fichtel C (2012) A 15-year perspective on the social organization and life history of sifaka in Kirindy forest. In: Kappeler PM, Watts DP (eds) Long-term field studies of primates. Springer, Berlin, pp 101-121

Kappeler PM, Cremer S, Nunn CL (2015) Sociality and health: impacts of sociality on disease susceptibility and transmission in animal and human societies. Philos Trans R Soc B 370:20140116. https://doi. org/10.1098/rstb.2014.0116

Koch F, Signer J, Kappeler PM, Fichtel C (2016) The role of the residence-effect on the outcome of intergroup encounters in Verreaux's sifakas. Sci Rep 6:28457. https://doi.org/10.1038/ srep 28457

Koch F, Ganzhorn JU, Rothman JM, Chapman CA, Fichtel C (2017) Sex and seasonal differences in diet and nutrient intake in Verreaux's sifakas (Propithecus verreauxi). Am J Primatol 79:e22595. https:// doi.org/10.1002/ajp.22595

Koenig A (2002) Competition for resources and its behavioral consequences among female primates. Int J Primatol 23:759-783. https://doi.org/10.1023/A:1015524931226

Koolhaas JM, Bartolomucci A, Buwalda B, de Boer SF, Flügge G, Korte SM, Meerlo P, Murison R, Olivier B, Palanza P, Richter-Levin G, Sgoifo A, Steimer T, Stiedl O, van Dijk G, Wöhr M, Fuchs E (2011) Stress revisited: a critical evaluation of the stress concept. Neurosci Biobehav Rev 35:1291-1301. https://doi.org/10.1016/j.neubiorev. 2011.02.003

Kruuk H, Parish T (1982) Factors affecting population density, group size and territory size of the European badger, Meles meles. J Zool 196: 31-39. https://doi.org/10.1111/j.1469-7998.1982.tb03492.x

Leimberger KG, Lewis RJ (2015) Patterns of male dispersal in Verreaux's sifaka (Propithecus verreauxi) at Kirindy Mitea National Park. Am J Primatol 79:e22455. https://doi.org/10.1002/ajp.22455
Majolo B, Huang P (2018) Group living. In: Vonk J, Shackelford T (eds) Encyclopedia of animal cognition and behavior. Springer International Publishing, Cham, pp 1-12

Majolo B, de Bortoli VA, Schino G (2008) Costs and benefits of group living in primates: group size effects on behaviour and demography. Anim Behav 76:1235-1247. https://doi.org/10.1016/j.anbehav. 2008.06.008

Markham AC, Gesquiere LR (2017) Costs and benefits of group living in primates: an energetic perspective. Philos Trans R Soc B 372: 20160239. https://doi.org/10.1098/rstb.2016.0239

Markham AC, Gesquiere LR, Alberts SC, Altmann J (2015) Optimal group size in a highly social mammal. Proc Natl Acad Sci USA 112:14882-14887. https://doi.org/10.1073/pnas.1517794112

Marsh CW (1981) Ranging behaviour and its relation to diet selection in Tana River Red colobus (Colobus badius rufomitratus). J Zool 195: 473-492. https://doi.org/10.1111/j.1469-7998.1981.tb03479.x

Martinez-Mota R (2015) The effects of habitat disturbance, host traits, and host physiology on patterns of gastrointestinal parasite infection in black howler monkeys (Alouatta pigra). Dissertation, University of Illinois at Urbana-Champaign

McEwen BS, Wingfield JC (2003) The concept of allostasis in biology and biomedicine. Horm Behav 43:2-15. https://doi.org/10.1016/ S0018-506X(02)00024-7

McLean KA, Trainor AM, Asner GP, Crofoot MC, Hopkins ME, Campbell CJ, Martin RE, Kapp DE, Jansen PA (2016) Movement patterns of three arboreal primates in a Neotropical moist forest explained by LiDAR-estimated canopy structure. Landsc Ecol 31: 1849-1862. https://doi.org/10.1007/s10980-016-0367-9

Michelena P, Pillot M-H, Henrion C, Toulet S, Boissy A, Bon R (2012) Group size elicits specific physiological response in herbivores. Biol Lett 8:537-539. https://doi.org/10.1098/rsbl.2012.0197

Mosser A, Packer C (2009) Group territoriality and the benefits of sociality in the African lion, Panthera leo. Anim Behav 78:359-370. https://doi.org/10.1016/j.anbehav.2009.04.024

Muehlenbein MP (2006) Intestinal parasite infections and fecal steroid levels in wild chimpanzees. Am J Phys Anthropol 130:546-550. https://doi.org/10.1002/ajpa.20391

Muehlenbein MP, Schwartz M, Richard A (2003) Parasitologic analyses of the sifaka (Propithecus verreauxi verreauxi) at Beza Mahafaly, Madagascar. J Zoo Wildlife Med 34:274-277. https://doi.org/10. 1638/1042-7260(2003)034[0274:PAOTSP]2.0.CO;2

Müller-Klein N, Heistermann M, Strube C, Morbach ZM, Lilie N, Franz M, Schülke O, Ostner J (2018) Physiological and social consequences of gastrointestinal nematode infection in a nonhuman primate. Behav Ecol (published online). https://doi.org/10.1093/ beheco/ary 168

Norbiato G, Bevilacqua M, Vago T, Taddei A, Clerici M (1997) Glucocorticoids and the immune function in the human immunodeficiency virus infection: a study in hypercortisolemic and cortisolresistant patients. J Clin Endocrinol Metab 82:3260-3263. https:// doi.org/10.1210/jcem.82.10.4304

Norscia I, Palagi E (2008) Berenty 2006: census of Propithecus verreauxi and possible evidence of population stress. Int J Primatol 29:1099 1115. https://doi.org/10.1007/s10764-008-9259-4

Norscia I, Carrai V, Borgognini-Tarli SM (2006) Influence of dry season and food quality and quantity on behavior and feeding strategy of Propithecus verreauxi in Kirindy, Madagascar. Int J Primatol 27: 1001-1022. https://doi.org/10.1007/s10764-006-9056-x

Nunn CL, Altizer S, Jones KE, Sechrest W (2003) Comparative tests of parasite species richness in primates. Am Nat 162:597-614. https:// doi.org/10.1086/378721

Patterson JEH, Ruckstuhl KE (2013) Parasite infection and host group size: a meta-analytical review. Parasitology 140:803-813. https:// doi.org/10.1017/S0031182012002259 
Pollard KA, Blumstein DT (2008) Time allocation and the evolution of group size. Anim Behav 76:1683-1699. https://doi.org/10.1016/j. anbehav.2008.08.006

Pride RE (2005) Optimal group size and seasonal stress in ring-tailed lemurs (Lemur catta). Behav Ecol 16:550-560. https://doi.org/10. 1093/beheco/ari025

QGIS Development Team (2018) QGIS geographic information system. Open Source Geospatial Foundation Project, http://qgis.osgeo.org. Accessed 25 June 2018

R Core Team (2018) R: a language and environment for statistical computing. R Foundation for Statistical Computing, Vienna http://www. R-project.org

Raouf SA, Smith LC, Brown MB, Wingfield JC, Brow CR (2006) Glucocorticoid hormone levels increase with group size and parasite load in cliff swallows. Anim Behav 71:39-48. https://doi.org/10. 1016/j.anbehav.2005.03.027

Rasambainarivo FT, Junge RE, Lewis RJ (2014) Biomedical evaluation of Verreaux's Sifaka (Propithecus verreauxi) from Kirindy Mitea National Park in Madagascar. J Zoo Wildlife Med 45:247-255. https://doi.org/10.1638/2013-0038R1.1

Rifkin JL, Nunn CL, Garamszegi LZ (2012) Do animals living in larger groups experience greater parasitism? A meta-analysis. Am Nat 180:70-82. https://doi.org/10.1086/666081

Romero LM (2002) Seasonal changes in plasma glucocorticoid concentrations in free-living vertebrates. Gen Comp Endocrinol 128:1-24. https://doi.org/10.1016/S0016-6480(02)00064-3

Romero LM, Dickens MJ, Cyr NE (2009) The reactive scope model - a new model integrating homeostasis, allostasis, and stress. Horm Behav 55:375-389. https://doi.org/10.1016/j.yhbeh.2008.12.009

Santos CD, Toldo MPA, Levy AMA, Prado JC (2008) Trypanosoma cruzi: effects of social stress in Calomys callosus a natural reservoir of infection. Exp Parasitol 119:197-201. https://doi.org/10.1016/j. exppara.2008.01.011

Sapolsky RM, Romero LM, Munck AU (2000) How do glucocorticoids influence stress responses? Integrating permissive, suppressive, stimulatory, and preparative actions. Endocr Rev 21:55-89. https:// doi.org/10.1210/edrv.21.1.0389

Scott CT (1998) Sampling methods for estimating change in forest resources. Ecol Appl 8:228-233. https://doi.org/10.1890/10510761(1998)008[0228:SMFECI]2.0.CO;2

Sheldon BC, Verhulst S (1996) Ecological immunology: costly parasite defences and trade-offs in evolutionary ecology. Trends Ecol Evol 11:317-321. https://doi.org/10.1016/0169-5347(96)10039-2

Skytte KA, Kirkegaard RH, Karst SM, Albertsen M (2018) ampvis2: an $\mathrm{R}$ package to analyse and visualise $16 \mathrm{~S}$ rRNA amplicon data. bioRxiv. https://doi.org/10.1101/299537

Snaith TV, Chapman CA, Rothman JM, Wasserman MD (2008) Bigger groups have fewer parasites and similar cortisol levels: a multi- group analysis in red colobus monkeys. Am J Primatol 70:10721080

Springer A, Kappeler PM (2016) Intestinal parasite communities of six sympatric lemur species at Kirindy Forest, Madagascar. Primate Biol 3:51-63. https://doi.org/10.5194/pb-3-51-2016

Srivathsan A, Ang A, Vogler AP, Meier R (2016) Fecal metagenomics for the simultaneous assessment of diet, parasites, and population genetics of an understudied primate. Front Zool 13:17. https://doi.org/ 10.1186/s12983-016-0150-4

Steenbeek R, van Schaik CP (2001) Competition and group size in Thomas's langurs (Presbytis thomasi): the folivore paradox revisited. Behav Ecol Sociobiol 49:100-110. https://doi.org/10. 1007/s002650000286

Strandburg-Peshkin A, Farine DR, Crofoot MC, Couzin ID (2017) Habitat and social factors shape individual decisions and emergent group structure during baboon collective movement. eLife 6:e19505

Sussman RW, Richard AF, Ratsirarson J, Sauther ML, Brockman DK, Gould L, Lawler R, Cuozzo FP (2012) Beza Mahafaly Special Reserve: long-term research on lemurs in southwestern Madagascar. In: Kappeler PM, Watts DP (eds) Long-term field studies of primates. Springer, Berlin, pp 45-66

Tanaka R, Hino A, Tsai IJ, Palomares-Rius JE, Yoshida A, Ogura Y, Hayashi T, Maruyama H, Kikuchi T (2014) Assessment of helminth biodiversity in wild rats using $18 \mathrm{~S}$ rDNA based metagenomics. PLoS One 9:e110769. https://doi.org/10.1371/journal.pone. 0110769

Terborgh J, Janson CH (1986) The socioecology of primate groups. Annu Rev Ecol Syst 17:111-136. https://doi.org/10.1146/annurev.es.17. 110186.000551

Treves A, Chapman CA (1996) Conspecific threat, predation avoidance, and resource defense: implications for grouping in langurs. Behav Ecol Sociobiol 39:43-53. https://doi.org/10.1007/s002650050265

Turnbull AV, Rivier CL (1999) Regulation of the hypothalamic-pituitaryadrenal axis by cytokines: actions and mechanisms of action. Physiol Rev 79:1-71. https://doi.org/10.1152/physrev.1999.79.1.1

Wimmer B, Craig BH, Pilkington JG, Pemberton JM (2004) Noninvasive assessment of parasitic nematode species diversity in wild Soay sheep using molecular markers. Int J Parasitol 34:625-631. https://doi.org/10.1016/j.jpara.2003.11.022

Wrangham RW, Gittleman JL, Chapman CA (1993) Constraints on group size in primates and carnivores: population density and day-range as assays of exploitation competition. Behav Ecol Sociobiol 32:199 209. https://doi.org/10.1007/BF00173778

Publisher's note Springer Nature remains neutral with regard to jurisdictional claims in published maps and institutional affiliations. 


\section{Appendix: Chapter 2}

This supplementary file contains more detailed information on:

a. Supplemental Methods

p. 28

A) Faecal glucocorticoid metabolite analyses

B) Parasite richness

C) Habitat quality

p. 30

D) Statistical analyses.

p. 30

b. Supplemental Results.

c. Further Analyses.

a. Results of GLMMs with group size measures, excluding infants

b. Results of correlations between core areas and group sizes.

c. Results of correlations among $\mathrm{fGCMs}$, activity and ranging patterns

p. 50

\section{1) Supplemental Methods}

\section{A) Faecal glucocorticoid metabolite (fGCM) analyses}

For fGCM analysis, faecal samples were extracted at the field site on the evening of the day the samples were collected. For steroid extraction, we manually homogenized all samples for three min and subsequently vortexed for $20 \mathrm{sec}$. Afterwards, samples were centrifuged with a manually operating centrifuge (c.f. Shutt et al. 2012; Rimbach et al. 2013) and $\sim 1.5 \mathrm{ml}$ of the supernatant were decanted into $2 \mathrm{ml}$ polypropylene safe-lock tubes (Eppendorf ${ }^{\circledR}$, Hamburg, Germany) for storage at ambient temperature in the dark (Rimbach et al., 2013). Faecal extracts were shipped to the Endocrinology Laboratory within one to six months following sample collection. Samples were stored at $-20^{\circ} \mathrm{C}$ until fGCM analysis. We measured fGCM concentrations from the faecal extracts by using a group-specific enzyme immunoassay (EIA) for the measurement of 5ß-reduced cortisol metabolites (for details see Heistermann et al. 2004). The assay has been proven to reliably assess adrenocortical activity from faecal samples of numerous primate species of all major taxa (e.g. Heistermann et al. 2006), including Verreaux's sifakas (Fichtel et al., 2007). Intra- and inter-assay coefficients of variations (CVs) of high- and low-quality controls were $7.1 \%$ (high, $n=17$ ) and $8.4 \%$ (low, $n=17$ ) and 9\% (high, $n=75$ ) and 16\% (low, $n=75)$, respectively. 


\section{B) Parasite richness}

Extraction of DNA, Amplification, and Sequencing of $18 \mathrm{~S}$ rRNA genes

We extracted DNA from approximately $100 \mathrm{mg}$ of faecal samples using the PowerSoil DNA isolation kit following the instructions of the manufacturer (MoBio, Carlsbad, Canada). However, to ensure complete homogenization of the faecal samples including parasite eggs, we used a FastPrep-24 ${ }^{\text {TM }}$ Classic Grinder (MP Biomedicals, Santa Ana, California, USA) instead of the suggested MO Bio Vortex adapter. Samples were homogenized for $20 \mathrm{~s}$ at $6.5 \mathrm{~m} / \mathrm{s}$. Eukaryotic $18 \mathrm{~S}$ rRNA gene amplicons were generated using the primers TAReuk454FWD1 and TAReukREV3 (Stoeck et al., 2010) harbouring the Illumina MiSeq sequencing adaptors. PCR reaction mixtures (total volume $50 \mu \mathrm{l}$ ) and contained $1 \mathrm{U}$ Phusion high fidelity DNA polymerase (Biozym Scientific, Oldendorf, Germany), $2.5 \mu$ l DMSO (5\%), $1 \mu$ l of forward and reverse 18S rRNA gene primers $(10 \mu \mathrm{M}), 1 \mu \mathrm{l}$ dNTP $(10 \mathrm{mM}), 0.2 \mu \mathrm{l} \mathrm{MgCl}(50 \mathrm{mM})$, and $50 \mathrm{ng}$ of isolated DNA. Thermal cycling conditions were as follows: initial denaturation for $1 \mathrm{~min}$ at $98^{\circ} \mathrm{C}, 25$ cycles at $98^{\circ} \mathrm{C}$ for $45 \mathrm{~s}, 60^{\circ} \mathrm{C}$ for $45 \mathrm{~s}$, and $72{ }^{\circ} \mathrm{C}$ for $30 \mathrm{~s}$, and a final extension at $72{ }^{\circ} \mathrm{C}$ for $5 \mathrm{~min}$. We included negative and positive controls (genomic DNA isolated from Aspergillus nidulans) in all PCRs. All PCR products were checked for appropriate size via gel electrophoresis. We performed PCR reactions in triplicates for each sample, then pooled in equimolar amounts and purified using MagSi-NGSPREP Plus (Steinbrenner, Wiesenbach, Germany) as described by the supplier. Nextera DNA Library Prep kits were used for indexing PCR products according to the manufacturer's manual (Illumina), followed by dual-indexed paired-end sequencing with the Illumina MiSeq platform ( 2 × 300 bp) and v3 chemistry.

$18 S$ rRNA gene amplicon analyses

We demultiplexed and clipped adapters from raw sequences using CASAVA data analysis software (Illumina). Paired-end sequences were merged using PEAR v0.9.11 with default parameters (Zhang et al., 2014). Afterwards, we removed sequences with average quality scores below 20 and/or containing unresolved bases using trimmomatic 0.36. (Caporaso et al., 2010). Additionally, we employed cut-adapt 1.18 with default settings to remove reverse and forward primer sequences (Martin, 2011). Amplicon Sequence Variants (ASVs) were generated with VSEARCH version 2.9.1 (Edgar, 2010). In detail, reads were sorted by length and amplicons with a read length shorter $250 \mathrm{bp}$ removed, afterwards, amplicons were dereplicated and denoised utilizing the UNOISE3 algorithm of VSEARCH. We removed chimeric sequences with VSEARCH using UCHIME3 (--uchime3_denovo) in de novo and reference (--uchime_ref) mode against the $P R^{2}$ database (version 4.11.0) (Guillou et al., 2013). Quality-filtered sequences were mapped to chimera-free ASVs and an ASV table was created with VSEARCH. Finally, we taxonomically classified ASVs with BLASTn against the $\mathrm{PR}^{2}$ database. 


\section{C) Habitat quality}

Forest inventories for six of the seven sifaka groups were taken in 2012 and, since the seventh group (Group M) entered the research area only by the end of 2016, another inventory was conducted in 2017 by the same field assistant. Even though sifaka home ranges are stable over years, some minor relocations still occur with time. Hence, after four years, home range location of one group (Group F1) had changed to a degree that only 6 out of 10 plots could be used for habitat comparisons. Altogether, the dataset contains 12177 trees within 66 plots.

\section{D) Statistical analyses}

We applied 10 generalized linear mixed-effect models (GLMMs) (Baayen et al., 2008) from the package the Ime4 package (version: 1.1.21) (Bates et al., 2012) with the optimizer "bobyqa" in R (version 3.4.4) (R Core Team, 2018), to test whether group size and group size-squared or group ID affect monthly measures of daily travel distances, home range sizes, and individual fGCMs. Response values were Intransformed to achieve roughly symmetric distributions and to avoid influential cases. In order to achieve easier interpretable models (Schielzeth, 2010) and to facilitate model convergence, we z-transformed (transformed to a mean of zero and a SD of one) all numerical variables, including predictors and random slopes. In models on group size effects, we included group size and group size-squared and their interaction with monthly temperature differences and mean monthly food availability. We included the interactions to examine group size effects on behavioural or physiological adaptations to seasonal changes. In models on the effects of group ID, no interaction was included. Monthly temperature differences, monthly food availability, study year (first or second) and sex (only for models on fGCMs) were included as fixed effects to examine ecological influences. Food availability was based on monitoring monthly phenology of 690 trees throughout the study period. We used a semi-quantitative method (Fournier, 1974) in which the availability for each plant part (i.e. leaves, fruit, flowers) was scored, ranging from 0 (complete absence) to 4 (maximum abundance) (for details, see Koch et al., 2017). Temperature differences describe the average monthly differences between daily highest and lowest temperatures. Both, food availability and temperature differences vary with season which is why we used them as proxies for seasonal effects on individual fGCMs (Figure S1a). We additionally controlled for study year (first or second). Group and animal ID (only for models on fGCMs) were utilized as random effects. To keep type I error rates at the nominal level of 5\%, we included random slopes (Barr et al., 2013). For models on home range size and daily travel distance, we included random slopes of study year, food availability and temperature differences within group. For the model on $\mathrm{fGCMs}$, we included the same random slopes within group and ID. Additional random slopes of group size and group size-squared were included within models testing group size effects. In models with fGCMs as response, we accounted for 
the number of collected samples per individual by utilizing the argument weights within the Ime4 package.

Assumptions for normality distributions and homoscedasticity were checked by visually inspecting a ggplot and residuals plotted against fitted values, respectively. We did not detect obvious deviations from the assumptions for any of the models. Model stability was assessed by excluding data points one by one and comparing the derived coefficients, using a function kindly provided by Roger Mundry. This revealed no obviously influential cases. Using the function vif of the R-package car (version 3.0-2) (Fox and Weisberg, 2011) we derived Variance Inflation Factors (VIF) (Field, 2009) to a standard linear model excluding random effects. No indication of issues with collinearity among the fixed factors was found (maximum Variance Inflation Factor among all models: 2.15; Quinn and Keough, 2002). We conducted comparisons between full and null models using likelihood ratio tests (R function ANOVA with argument test set to "Chisq") (Dobson, 2002; Forstmeier and Schielzeth, 2011). Null models contained only intercepts, random effects and random slopes. We fitted the models using Maximum Likelihood rather than Restricted Maximum Likelihood (Bolker et al., 2009) to allow for likelihood ratios tests. Pvalues for individual effects were based on likelihood ratio tests comparing the full with respective null models using the drop1 function (Barr et al., 2013). If models resulted in significant effects of group ID, we further analysed the effect by conducting multiple comparisons of means using Tukey contrasts with the glht function of the package multcomp (version 1.4-10). Effect sizes for the entirety of fixed and random effects of the full models were obtained with the function r.squared GLMM of the package MuMIn (version 1.42.1) (Barton, 2018). We assessed confidence intervals with parametric bootstrapping using an adjusted function, which is based on the function bootMer from the Ime4 package and was provided by Roger Mundry.

To examine effects of group size or group ID on mean monthly foraging and resting rates, we conducted binomial models with beta error distribution structures and a logit link functions using the glmmTMB package (version 0.2.3) (Brooks et al., 2017). Response variables (foraging and resting rates), predictors (group size or group ID, food availability, temperature differences), fixed effects (study year), random effects (group and animal ID) and random slopes (food availability, temperature differences, study year, group size (in models investigating group size effects) within group and animal ID) were included the same way as described above. Due to convergence issues, we could not include group sizesquared as predictor variables and, therefore, not investigate polynomial group size effects. Interactions of food availability and temperature differences with linear group sizes were included and treated as described above. We encountered no issues when checking for model stability, collinearity (maximum Variance Inflation Factor among all models: 2.20) and overdispersion. Full-null model comparisons and estimations of $p$-values were conducted as described above. Null models contained only intercepts, fixed effects (i.e. study year), random effects and random slopes. Models resulting in significant effects of 
group ID were further analysed by comparing predicted marginal means using the Ismeans function of the package emmeans (version 1.3.3). We assessed confidence intervals with the confint function of the glmmTMB package.

\section{2) Supplemental Results}

\section{Ranging Patterns}

\section{Home Range Size}

Table S1 Influence of group size on home range size (In-transformed) in seven groups of Verreaux's sifakas; results of the full model $\left(G L M M\right.$; N $\left.N_{\text {observations }}=143, N_{I D}=41\right)$.

\begin{tabular}{|c|c|c|c|c|c|c|c|c|c|}
\hline Term & Est & SE & Lower $\mathrm{Cl}$ & Upper Cl & $\operatorname{Min}^{a}$ & $\operatorname{Max}^{a}$ & $\chi^{2 b}$ & $d f$ & $P$ \\
\hline (Intercept) & 2.682 & 0.114 & 2.448 & 2.889 & 2.574 & 2.775 & c & c & c \\
\hline Year $^{d}$ & -0.049 & 0.036 & -0.121 & 0.020 & -0.065 & -0.018 & 1.693 & 1 & 0.193 \\
\hline$\Delta$ Temp $^{\mathrm{e}}$ & -0.270 & 0.034 & -0.339 & -0.202 & -0.304 & -0.246 & c & c & c \\
\hline Food availability ${ }^{f}$ & -0.217 & 0.040 & -0.293 & -0.130 & -0.237 & -0.180 & c & c & c \\
\hline Group sizeg & 0.071 & 0.072 & -0.084 & 0.221 & -0.064 & 0.148 & c & c & c \\
\hline Group size $2 \mathrm{~g}$ & -0.043 & 0.041 & -0.131 & 0.046 & -0.247 & 0.009 & c & c & c \\
\hline$\Delta$ Temp*Group size & -0.054 & 0.032 & -0.118 & 0.010 & -0.076 & 0.017 & 2.649 & 1 & 0.104 \\
\hline$\Delta$ Temp*Group size ${ }^{2}$ & 0.026 & 0.020 & -0.013 & 0.068 & -0.014 & 0.140 & 1.647 & 1 & 0.199 \\
\hline Food avail*Group size & -0.017 & 0.037 & -0.089 & 0.061 & -0.027 & 0.036 & 0.245 & 1 & 0.621 \\
\hline Food avail*Group size ${ }^{2}$ & 0.021 & 0.024 & -0.029 & 0.069 & -0.001 & 0.112 & 0.688 & 1 & 0.407 \\
\hline 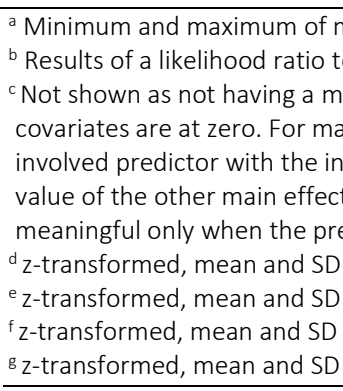 & \multicolumn{8}{|c|}{$\begin{array}{l}\text { 'Results of a likelihood ratio test comparing the full model with a reduced model lacking the respective term } \\
\text { c Not shown as not having a meaningful interpretation. For intercepts, p-values would refer to estimated fGCM concentrations, when all } \\
\text { involved predictor with the interacting covariate at zero. This means the main effects of predictors involved in interactions depend on the } \\
\text { value of the other main effects and are, therefore, not interpretable in themselves. Therefore, we consider P values of main effects to be } \\
\text { meaningful only when the predictors are not involved in an interaction. }\end{array}$} & \\
\hline
\end{tabular}


Table S2 Influence of group size on home range size (In-transformed) in seven groups of Verreaux's sifakas; results of the full model $\left(G L M M\right.$; Nobservations $\left.=143, N_{I D}=41\right)$.

\begin{tabular}{|c|c|c|c|c|c|c|c|c|c|c|}
\hline Term & & Est & SE & Lower $\mathrm{Cl}$ & Upper Cl & $\operatorname{Min}^{a}$ & $\operatorname{Max}^{\mathrm{a}}$ & $\chi^{2 b}$ & $d f$ & $P$ \\
\hline (Intercept) & & 2.552 & 0.039 & 2.476 & 2.629 & 2.552 & 2.639 & c & $\mathrm{c}$ & c \\
\hline \multirow[t]{6}{*}{ Group } & F & 0.087 & 0.055 & -0.019 & 0.197 & 0.086 & 0.087 & $32.95^{d}$ & $6^{d}$ & $<0.001^{d}$ \\
\hline & $\mathrm{F} 1$ & 0.024 & 0.055 & -0.076 & 0.127 & -0.063 & 0.024 & c & c & c \\
\hline & G & -0.090 & 0.055 & -0.198 & 0.020 & -0.177 & -0.090 & c & c & c \\
\hline & J & -0.309 & 0.061 & -0.437 & -0.196 & -0.398 & -0.303 & c & c & c \\
\hline & $\mathrm{L}$ & 0.377 & 0.056 & 0.266 & 0.488 & 0.290 & 0.378 & c & c & c \\
\hline & M & 0.552 & 0.061 & 0.434 & 0.676 & 0.470 & 0.558 & c & c & c \\
\hline$\Delta$ Temp $^{\mathrm{e}}$ & & -0.251 & 0.024 & -0.298 & -0.207 & -0.263 & -0.240 & 19.656 & 1 & $<0.001$ \\
\hline Food availability & & -0.203 & 0.031 & -0.266 & -0.147 & -0.215 & -0.180 & 15.305 & 1 & $<0.001$ \\
\hline Yearg & & -0.044 & 0.032 & -0.107 & 0.018 & -0.061 & -0.016 & 1.667 & 1 & 0.197 \\
\hline $\begin{array}{l}\text { a Minimum and max } \\
\text { b Results of a likelihg } \\
\text { ' Not shown as not } r \\
{ }^{\mathrm{d}} \text { Values refer to the } \\
\text { e } \mathrm{z} \text {-transformed, me } \\
{ }^{\mathrm{f}} \text {-transformed, me } \\
\mathrm{g}_{\text {z-transformed, me }}\end{array}$ & $\begin{array}{l}\text { Imum } \\
\text { od ra } \\
\text { aving } \\
\text { over } \\
\text { an an } \\
\text { in an }\end{array}$ & $\begin{array}{l}f \text { model es } \\
\text { test comp } \\
\text { meaningfu } \\
\text { test of the } \\
\text { SD of the o } \\
\text { D of the or }\end{array}$ & $\begin{array}{l}\text { mates obt } \\
\text { ring the } f \\
\text { interpreta } \\
\text { ffect of th } \\
\text { sinal value } \\
\text { sinal value }\end{array}$ & $\begin{array}{l}\text { ned when drc } \\
\text { model with } \\
\text { on. See footn } \\
\text { predictor (“G } \\
\text { were } 16.398 \\
\text { were } 2.811 \text { a } \\
\text { were } 1.503 \text { a }\end{array}$ & $\begin{array}{l}\text { ping levels of } \\
\text { educed mode } \\
\text { es of Table S1 } \\
\text { up"), not the } \\
\text { d } 4.269 \text {, resp } \\
0.967 \text {, respe }\end{array}$ & $\begin{array}{l}\text { ndom effec } \\
\text { acking the } \\
\text { or details. } \\
\text { ecific level } \\
\text { tively } \\
\text { vely } \\
\text { ively }\end{array}$ & $\begin{array}{l}\text { one at a ti } \\
\text { pective te } \\
\text { dicated in }\end{array}$ & respective & ow & 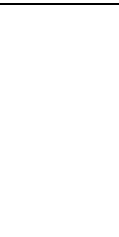 \\
\hline
\end{tabular}

\section{Daily Travel Distances}

Table S3 Influence of group size on daily travel distance (In-transformed) in seven groups of Verreaux's sifakas; results of the full model (GLMM; $\left.N_{\text {Observations }}=143, N_{I D}=41\right)$.

\begin{tabular}{|c|c|c|c|c|c|c|c|c|c|}
\hline Term & Est & SE & Lower $\mathrm{Cl}$ & Upper Cl & $\operatorname{Min}^{a}$ & $\operatorname{Max}^{a}$ & $\chi^{2 b}$ & $d f$ & $P$ \\
\hline (Intercept) & 6.818 & 0.031 & 6.752 & 6.879 & 6.786 & 6.861 & c & c & c \\
\hline Year & -0.048 & 0.020 & -0.085 & -0.008 & -0.057 & -0.027 & 4.285 & 1 & 0.038 \\
\hline$\Delta$ Temp $^{\mathrm{e}}$ & -0.284 & 0.017 & -0.317 & -0.250 & -0.305 & -0.273 & c & c & c \\
\hline Food availability ${ }^{f}$ & -0.159 & 0.017 & -0.194 & -0.126 & -0.168 & -0.145 & c & c & c \\
\hline Group size $e^{g}$ & 0.020 & 0.027 & -0.039 & 0.078 & -0.027 & 0.038 & c & c & c \\
\hline Group size $\mathrm{s}^{2}$ & 0.006 & 0.018 & -0.032 & 0.050 & -0.024 & 0.030 & c & c & c \\
\hline$\Delta$ Temp*Group size & -0.042 & 0.016 & -0.074 & -0.011 & -0.060 & 0.014 & 6.623 & 1 & 0.010 \\
\hline$\Delta$ Temp*Group size ${ }^{2}$ & 0.021 & 0.011 & -0.001 & 0.043 & -0.008 & 0.082 & 3.791 & 1 & 0.052 \\
\hline Food avail*Group size & -0.026 & 0.016 & -0.060 & 0.007 & -0.035 & 0.003 & 2.422 & 1 & 0.120 \\
\hline Food avail*Group size ${ }^{2}$ & 6.818 & 0.031 & -0.007 & 0.036 & 0.003 & 0.041 & 1.769 & 1 & 0.183 \\
\hline \multicolumn{10}{|c|}{$\begin{array}{l}\text { a Minimum and maximum of model estimates obtained when dropping levels of random } \\
\text { b Results of a likelihood ratio test comparing the full model with a reduced model lacking } \\
\text { c Not shown as not having a meaningful interpretation. See footnotes of Table S1 for deta } \\
{ }^{d} z \text {-transformed, mean and SD of the original values were } 1.503 \text { and } 0.502 \text {, respectively } \\
\text { ez-transformed, mean and SD of the original values were } 16.398 \text { and } 4.269 \text {, respectively } \\
{ }^{f} z \text {-transformed, mean and SD of the original values were } 2.811 \text { and } 0.967 \text {, respectively } \\
{ }^{8} \text { z-transformed, mean and SD of the original values were } 5.790 \text { and } 2.072 \text {, respectively }\end{array}$} \\
\hline
\end{tabular}


Table S4 Influence of group ID on daily travel distance (In-transformed) in seven groups of Verreaux's sifakas; results of the full model (GLMM; Nobservations $\left.=143, N_{I D}=41\right)$.

\begin{tabular}{|c|c|c|c|c|c|c|c|c|c|c|}
\hline Term & & Est & SE & Lower Cl & Upper Cl & $\operatorname{Min}^{a}$ & $\operatorname{Max}^{a}$ & $\chi^{2 b}$ & $d f$ & $P$ \\
\hline (Intercept) & & 6.853 & 0.022 & 6.807 & 6.896 & 6.853 & 6.865 & c & c & c \\
\hline \multirow[t]{6}{*}{ Group } & $F$ & 0.012 & 0.031 & -0.049 & 0.073 & 0.012 & 0.012 & $22.670^{d}$ & $6^{d}$ & $0.001^{d}$ \\
\hline & F1 & -0.080 & 0.031 & -0.137 & -0.020 & -0.092 & -0.079 & c & c & c \\
\hline & G & -0.041 & 0.031 & -0.099 & 0.019 & -0.053 & -0.041 & c & c & c \\
\hline & $J$ & -0.176 & 0.034 & -0.244 & -0.109 & -0.189 & -0.170 & c & c & c \\
\hline & $\mathrm{L}$ & 0.033 & 0.031 & -0.022 & 0.096 & 0.020 & 0.034 & c & c & c \\
\hline & $M$ & 0.051 & 0.034 & -0.018 & 0.122 & 0.043 & 0.055 & c & c & c \\
\hline$\Delta$ Temp $^{\mathrm{e}}$ & & -0.259 & 0.014 & -0.289 & -0.231 & -0.275 & -0.251 & 30.813 & 1 & $<0.001$ \\
\hline Food availability & & -0.143 & 0.013 & -0.167 & -0.116 & -0.151 & -0.132 & 25.954 & 1 & $<0.001$ \\
\hline Year' & & -0.051 & 0.020 & -0.086 & -0.012 & -0.060 & -0.031 & 4.694 & 1 & 0.030 \\
\hline $\begin{array}{l}\text { a Minimum and max } \\
\text { b Results of a likeliho } \\
\text { c Not shown as not } ~ \\
{ }^{\mathrm{d}} \text { Values refer to the } \\
\mathrm{e}_{\mathrm{z} \text {-transformed, me }} \\
{ }_{\mathrm{f}} \mathrm{\text {-transformed,med }} \\
\mathrm{g}_{\mathrm{z} \text {-transformed, me }}\end{array}$ & $\begin{array}{l}\text { imum } \\
\text { od ra } \\
\text { laving } \\
\text { over } \\
\text { an an } \\
\text { an anc }\end{array}$ & $\begin{array}{l}\text { f model es } \\
\text { test comp } \\
\text { meaningfu } \\
\text { test of the } \\
\text { SD of the o } \\
\text { D of the or } \\
\text { SD of the o }\end{array}$ & $\begin{array}{l}\text { mates obt } \\
\text { aring the fu } \\
\text { interpreta } \\
\text { ffect of th } \\
\text { ginal value } \\
\text { sinal value } \\
\text { ginal value }\end{array}$ & $\begin{array}{l}\text { ned when drc } \\
\text { I model with a } \\
\text { on. See footn } \\
\text { predictor ("G } \\
\text { were } 16.398 \\
\text { were } 2.811 \text { ar } \\
\text { were } 1.503 \mathrm{a} \\
\end{array}$ & $\begin{array}{l}\text { ping levels of } \\
\text { educed mode } \\
\text { es of Table S1 } \\
\text { up"), not the } \\
\text { d 4.269, resp } \\
0.967 \text {, respe } \\
0.502 \text {, respe }\end{array}$ & $\begin{array}{l}\text { ndom effec } \\
\text { acking the r } \\
\text { or details. } \\
\text { eecific level } \\
\text { tively } \\
\text { ively } \\
\text { ively }\end{array}$ & $\begin{array}{l}\text { s one at a } t \\
\text { spective te } \\
\text { dicated in }\end{array}$ & $\begin{array}{l}\text { ne } \\
\text { m }\end{array}$ & ow & \\
\hline
\end{tabular}


Table S5 Results of post-hoc multiple comparisons of means using Tukey contrasts with adjusted p-values (Bonferroni) for comparisons of home range sizes, core areas and daily travel distances among groups. Significant p-values are highlighted in bold.

\begin{tabular}{|c|c|c|c|c|c|c|}
\hline \multirow{2}{*}{ Groups } & \multicolumn{2}{|c|}{ Home range size } & \multicolumn{2}{|c|}{ Core areas* } & \multicolumn{2}{|c|}{ Daily travel distance } \\
\hline & Z & $p$ adj & Z & $p$ adj & Z & $p$ adj \\
\hline$F-E$ & 1.570 & 1.000 & -0.403 & 1.000 & 0.389 & 1.000 \\
\hline$F 1-E$ & 0.441 & 1.000 & -0.854 & 1.000 & -2.568 & 0.215 \\
\hline$F 1-F$ & -1.129 & 1.000 & -0.451 & 1.000 & -2.957 & 0.065 \\
\hline$G-E$ & -1.631 & 1.000 & -1.213 & 1.000 & -1.310 & 1.000 \\
\hline$G-F$ & -3.202 & 0.029 & -0.811 & 1.000 & -1.699 & 1.000 \\
\hline G - F1 & -2.073 & 0.802 & -0.359 & 1.000 & 1.257 & 1.000 \\
\hline$J-E$ & -5.088 & $<0.001$ & -2.653 & 0.168 & -5.147 & $<0.001$ \\
\hline$J-F$ & -6.517 & $<0.001$ & -2.281 & 0.474 & -5.499 & $<0.001$ \\
\hline$J-F 1$ & -5.489 & $<0.001$ & -1.864 & 1.000 & -2.819 & 0.101 \\
\hline$J-G$ & -3.602 & 0.007 & -1.532 & 1.000 & -3.959 & 0.002 \\
\hline$L-E$ & 6.727 & $<0.001$ & 5.185 & $<0.001$ & 1.045 & 1.000 \\
\hline$L-F$ & 5.178 & $<0.001$ & 5.583 & $<0.001$ & 0.661 & 1.000 \\
\hline $\mathrm{L}-\mathrm{F} 1$ & 6.292 & $<0.001$ & 6.029 & $<0.001$ & 3.579 & 0.007 \\
\hline$L-G$ & 8.338 & $<0.001$ & 6.384 & $<0.001$ & 2.338 & 0.407 \\
\hline$L-J$ & 11.186 & $<0.001$ & 7.431 & $<0.001$ & 6.046 & $<0.001$ \\
\hline$M-E$ & 9.061 & $<0.001$ & 5.538 & $<0.001$ & 1.484 & 1.000 \\
\hline$M-F$ & 7.637 & $<0.001$ & 5.909 & $<0.001$ & 1.134 & 1.000 \\
\hline$M-F 1$ & 8.661 & $<0.001$ & 6.325 & $<0.001$ & 3.794 & 0.003 \\
\hline$M-G$ & 10.541 & $<0.001$ & 6.657 & $<0.001$ & 2.663 & 0.163 \\
\hline$M-J$ & 12.936 & $<0.001$ & 7.567 & $<0.001$ & 6.024 & $<0.001$ \\
\hline$M-L$ & 2.844 & 0.094 & 0.688 & 1.000 & 0.526 & 1.000 \\
\hline
\end{tabular}




\section{Daily Activities}

\section{Foraging}

Table S6 Influence of group size on foraging rates in seven groups of Verreaux's sifakas; results of the full model (GLMM; Nobservations $\left.=659, N_{I D}=41\right)$.

\begin{tabular}{|c|c|c|c|c|c|c|c|c|c|}
\hline Term & Est & SE & Lower $\mathrm{Cl}$ & Upper Cl & $\operatorname{Min}^{a}$ & $\operatorname{Max}^{a}$ & $\chi^{2 b}$ & $d f$ & $P$ \\
\hline (Intercept) & -0.257 & 0.051 & -0.358 & -0.157 & -0.309 & -0.210 & c & c & c \\
\hline Year $^{d}$ & 0.098 & 0.044 & 0.012 & 0.184 & 0.064 & 0.124 & 4.775 & 1 & 0.029 \\
\hline$\Delta$ Temp $^{\mathrm{e}}$ & -0.119 & 0.089 & -0.293 & 0.054 & -0.207 & 0.107 & c & c & c \\
\hline Food availability ${ }^{f}$ & -0.049 & 0.052 & -0.152 & 0.053 & -0.108 & 0.049 & c & c & c \\
\hline Group size ${ }^{g}$ & 0.123 & 0.092 & -0.057 & 0.304 & 0.077 & 0.187 & c & c & c \\
\hline$\Delta$ Temp*Group size & 0.124 & 0.088 & -0.048 & 0.296 & -0.021 & 0.406 & 2.237 & 1 & 0.135 \\
\hline Food avail*Group size & 0.018 & 0.055 & -0.089 & 0.125 & -0.030 & 0.151 & 0.106 & 1 & 0.745 \\
\hline \multicolumn{10}{|c|}{$\begin{array}{l}\text { a Minimum and maximum of model estimates obtained when dropping levels of random } \\
\text { b Results of a likelihood ratio test comparing the full model with a reduced model lacking } \\
\text { ' Not shown as not having a meaningful interpretation. See footnotes of Table S1 for deta } \\
{ }^{d} z \text {-transformed, mean and SD of the original values were } 1.525 \text { and } 0.500 \text {, respectively } \\
\text { e-transformed, mean and SD of the original values were } 16.687 \text { and } 4.278 \text {, respectively } \\
\text { f } z \text {-transformed, mean and SD of the original values were } 2.803 \text { and } 0.973 \text {, respectively } \\
{ }^{g} z \text {-transformed, mean and SD of the original values were } 6.501 \text { and } 2.169 \text {, respectively }\end{array}$} \\
\hline
\end{tabular}

Table S7 Influence of group ID on foraging rates in seven groups of Verreaux's sifakas; results of the full model (GLMM; N Nobservations $=659, N_{I D}=41$ ).

\begin{tabular}{|c|c|c|c|c|c|c|c|c|c|c|}
\hline Term & & Est & SE & Lower $\mathrm{Cl}$ & Upper Cl & $\operatorname{Min}^{a}$ & $\operatorname{Max}^{a}$ & $\chi^{2 b}$ & $d f$ & $P$ \\
\hline (Intercept) & & -0.224 & 0.088 & -0.397 & -0.051 & -0.260 & -0.138 & c & c & c \\
\hline \multirow[t]{6}{*}{ Group } & $\mathrm{F}$ & 0.084 & 0.114 & -0.140 & 0.308 & 0.038 & 0.154 & $17.009^{d}$ & $6^{d}$ & $0.009^{d}$ \\
\hline & $\mathrm{F} 1$ & -0.498 & 0.164 & -0.818 & -0.177 & -0.659 & -0.356 & c & c & c \\
\hline & $G$ & -0.291 & 0.135 & -0.555 & -0.027 & -0.381 & -0.203 & c & c & c \\
\hline & $J$ & -0.092 & 0.124 & -0.336 & 0.151 & -0.177 & 0.007 & c & c & c \\
\hline & $\mathrm{L}$ & 0.191 & 0.138 & -0.079 & 0.462 & -0.263 & -0.047 & c & c & c \\
\hline & $M$ & -0.167 & 0.152 & -0.466 & 0.131 & -0.260 & -0.138 & c & c & c \\
\hline$\Delta$ Temp $^{\mathrm{e}}$ & & -0.178 & 0.085 & -0.345 & -0.011 & -0.267 & -0.098 & 3.608 & 1 & 0.058 \\
\hline Food availability & & -0.087 & 0.051 & -0.188 & 0.013 & -0.157 & -0.056 & 2.788 & 1 & 0.095 \\
\hline Year ${ }^{\mathrm{g}}$ & & 0.125 & 0.046 & 0.035 & 0.214 & 0.089 & 0.161 & 6.691 & 1 & 0.010 \\
\hline $\begin{array}{l}\text { a Minimum and max } \\
\text { b Results of a likeliho } \\
\text { c Not shown as not } \mathrm{h} \\
\text { d Values refer to the } \\
\text { e z-transformed, me } \\
{ }^{\mathrm{f}} \text {-transformed, mea } \\
\mathrm{g}_{\mathrm{z} \text {-transformed, me }}\end{array}$ & $\begin{array}{l}\text { imum } \\
\text { od ra } \\
\text { laving } \\
\text { over } \\
\text { an an } \\
\text { an anc } \\
\text { an an }\end{array}$ & $\begin{array}{l}\text { f model es } \\
\text { o test comp } \\
\text { meaningfy } \\
\text { test of the } \\
\text { SD of the o } \\
\text { SD of the or } \\
\text { SD of the o }\end{array}$ & $\begin{array}{l}\text { nates obt } \\
\text { ring the fu } \\
\text { interpreta } \\
\text { ffect of th } \\
\text { sinal value } \\
\text { inal value } \\
\text { sinal value } \\
\end{array}$ & $\begin{array}{l}\text { ned when dr } \\
\text { I model with } \\
\text { on. See footr } \\
\text { predictor ("C } \\
\text { were } 16.687 \\
\text { were } 2.803 \text { a } \\
\text { were } 1.525 \text { a }\end{array}$ & $\begin{array}{l}\text { ping levels of } \\
\text { educed mod } \\
\text { es of Table S } \\
\text { up"), not the } \\
\text { d 4.278, resh } \\
0.973 \text {, respe } \\
0.500 \text {, respe }\end{array}$ & $\begin{array}{l}\text { indom effe } \\
\text { acking the } \\
\text { or details. } \\
\text { eecific leve } \\
\text { ctively } \\
\text { ively } \\
\text { ively }\end{array}$ & $\begin{array}{l}\text { s one at a } \\
\text { spective } t \\
\text { ndicated in }\end{array}$ & $\begin{array}{l}\text { ne } \\
\text { he respectiv }\end{array}$ & row & \\
\hline
\end{tabular}




\section{Resting}

Table S8 Influence of group size on resting rates in seven groups of Verreaux's sifakas; results of the full model (GLMM; Nobservations $=659, \mathrm{~N}_{\mathrm{ID}}=41$ ).

\begin{tabular}{|c|c|c|c|c|c|c|c|c|c|}
\hline Term & Est & SE & Lower $\mathrm{Cl}$ & Upper Cl & $\operatorname{Min}^{a}$ & $\operatorname{Max}^{a}$ & $\chi^{2 b}$ & $d f$ & $P$ \\
\hline (Intercept) & -0.032 & 0.048 & -0.126 & 0.061 & -0.085 & 0.020 & c & c & c \\
\hline Year $^{d}$ & -0.066 & 0.034 & -0.133 & 0.001 & -0.086 & -0.048 & 3.685 & 1 & 0.055 \\
\hline$\Delta$ Temp $^{\mathrm{e}}$ & 0.110 & 0.071 & -0.029 & 0.249 & -0.074 & 0.184 & c & c & c \\
\hline Food availability ${ }^{f}$ & 0.012 & 0.048 & -0.082 & 0.107 & -0.056 & 0.060 & c & c & c \\
\hline Group size ${ }^{g}$ & -0.110 & 0.086 & -0.279 & 0.058 & -0.171 & -0.070 & c & c & c \\
\hline$\Delta$ Temp*Group size & -0.070 & 0.074 & -0.214 & 0.074 & -0.300 & 0.027 & 0.990 & 1 & 0.320 \\
\hline Food avail*Group size & -0.008 & 0.050 & -0.106 & 0.091 & -0.100 & 0.020 & 0.024 & 1 & 0.878 \\
\hline \multicolumn{10}{|c|}{ 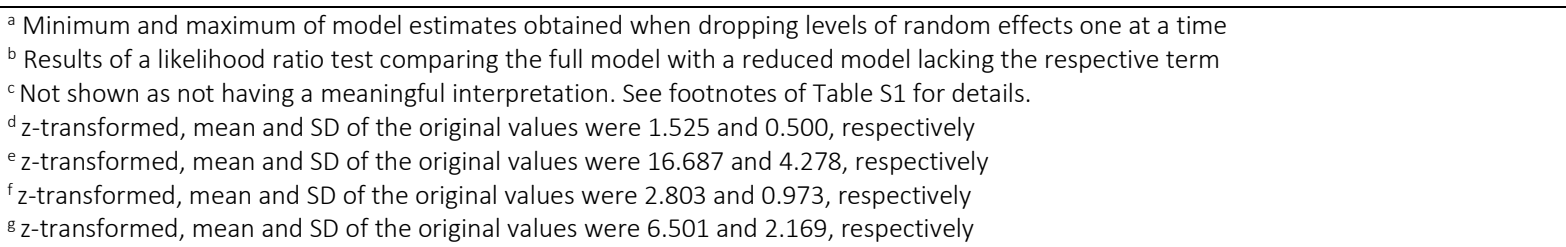 } \\
\hline
\end{tabular}

Table S9 Influence of group ID on resting rates in seven groups of Verreaux's sifakas; results of the full model (GLMM; N Nobservations $=659, N_{I D}=41$ ).

\begin{tabular}{|c|c|c|c|c|c|c|c|c|c|c|}
\hline Term & & Est & SE & Lower $\mathrm{Cl}$ & Upper Cl & $\operatorname{Min}^{a}$ & $\operatorname{Max}^{a}$ & $\chi^{2 b}$ & $d f$ & $P$ \\
\hline (Intercept) & & -0.060 & 0.082 & -0.220 & 0.100 & -0.155 & -0.032 & c & c & c \\
\hline \multirow[t]{6}{*}{ Group } & $\mathrm{F}$ & -0.090 & 0.105 & -0.295 & 0.115 & -0.157 & -0.047 & $17.358^{d}$ & $6^{d}$ & $0.008^{d}$ \\
\hline & $\mathrm{F} 1$ & 0.452 & 0.150 & 0.159 & 0.746 & 0.311 & 0.563 & c & c & c \\
\hline & G & 0.259 & 0.124 & 0.015 & 0.503 & 0.150 & 0.356 & c & c & c \\
\hline & J & 0.089 & 0.115 & -0.135 & 0.314 & 0.018 & 0.186 & c & c & c \\
\hline & L & -0.207 & 0.127 & -0.456 & 0.043 & 0.115 & 0.249 & c & c & c \\
\hline & $M$ & 0.157 & 0.136 & -0.109 & 0.422 & -0.155 & -0.032 & c & c & c \\
\hline$\Delta$ Temp $^{\mathrm{e}}$ & & 0.149 & 0.066 & 0.019 & 0.278 & 0.096 & 0.220 & 4.202 & 1 & 0.040 \\
\hline Food availability & & 0.043 & 0.047 & -0.050 & 0.135 & 0.013 & 0.098 & 0.820 & 1 & 0.365 \\
\hline Year' & & -0.089 & 0.033 & -0.155 & -0.024 & -0.104 & -0.072 & 6.969 & 1 & 0.008 \\
\hline $\begin{array}{l}\text { a Minimum and max } \\
\text { b Results of a likelihd } \\
{ }^{\mathrm{c}} \text { Not shown as not } \\
\mathrm{d}^{\mathrm{a}} \text { Values refer to the } \\
\mathrm{e}_{\mathrm{z} \text {-transformed, me }} \\
{ }^{\mathrm{f}} \text {-transformed, mea } \\
\mathrm{g}_{\mathrm{Z} \text {-transformed, me }}\end{array}$ & $\begin{array}{l}\text { mum } \\
\text { od ra } \\
\text { aving } \\
\text { overa } \\
\text { an anc } \\
\text { in and } \\
\text { an anc }\end{array}$ & $\begin{array}{l}\text { f model est } \\
\text { test comp } \\
\text { meaningfu } \\
\text { test of the } \\
\text { D of the or } \\
D \text { of the or } \\
\text { D of the or }\end{array}$ & $\begin{array}{l}\text { mates obt } \\
\text { ring the } f \\
\text { interpreta } \\
\text { ffect of th } \\
\text { ginal value } \\
\text { inal value } \\
\text { sinal value }\end{array}$ & $\begin{array}{l}\text { ned when drc } \\
\text { model with } \\
\text { on. See footn } \\
\text { predictor (“ } G \\
\text { were } 16.687 \\
\text { were } 2.803 \text { a } \\
\text { were } 1.525 \text { a }\end{array}$ & $\begin{array}{l}\text { oing levels of } \\
\text { educed mode } \\
\text { es of Table S. } \\
\text { up"), not the } \\
\text { d } 4.278 \text {, resp } \\
0.973 \text {, respe } \\
0.500 \text {, respe }\end{array}$ & $\begin{array}{l}\text { ndom effec } \\
\text { acking the } \\
\text { or details. } \\
\text { ecific level } \\
\text { tively } \\
\text { vely } \\
\text { ively }\end{array}$ & $\begin{array}{l}\text { one at a t } \\
\text { spective te } \\
\text { dicated in }\end{array}$ & $\begin{array}{l}\text { ne } \\
\text { me respectiv }\end{array}$ & row & \\
\hline
\end{tabular}


Table S10 Results of post-hoc comparisons of predicted marginal means with adjusted p-values (Tukey) for comparisons of foraging and resting rates among groups. Significant $p$-values are highlighted in bold.

\begin{tabular}{|c|c|c|c|c|}
\hline \multirow{2}{*}{ Groups } & \multicolumn{2}{|c|}{ Foraging rates } & \multicolumn{2}{|c|}{ Resting rates } \\
\hline & Estimate & $p$ adj & Estimate & $\mathrm{p}$ adj \\
\hline$E-F$ & -0.084 & 0.991 & 0.090 & 0.978 \\
\hline$E-F 1$ & 0.498 & 0.039 & -0.453 & 0.042 \\
\hline$E-G$ & 0.291 & 0.319 & -0.259 & 0.363 \\
\hline$E-J$ & 0.092 & 0.990 & -0.089 & 0.987 \\
\hline$E-L$ & -0.191 & 0.810 & 0.207 & 0.667 \\
\hline$E-M$ & 0.167 & 0.929 & -0.157 & 0.910 \\
\hline$F-F 1$ & 0.581 & 0.004 & -0.542 & 0.003 \\
\hline$F-G$ & 0.375 & 0.044 & -0.349 & 0.038 \\
\hline$F-J$ & 0.176 & 0.707 & -0.179 & 0.599 \\
\hline$F-L$ & -0.108 & 0.979 & 0.117 & 0.955 \\
\hline$F-M$ & 0.251 & 0.589 & -0.247 & 0.449 \\
\hline$F 1-G$ & -0.207 & 0.893 & 0.193 & 0.882 \\
\hline F1 - J & -0.406 & 0.168 & 0.363 & 0.187 \\
\hline $\mathrm{F} 1-\mathrm{L}$ & -0.689 & 0.002 & 0.659 & 0.001 \\
\hline $\mathrm{F} 1-\mathrm{M}$ & -0.330 & 0.559 & 0.296 & 0.559 \\
\hline$G-J$ & -0.199 & 0.755 & 0.170 & 0.815 \\
\hline$G-L$ & -0.482 & 0.019 & 0.466 & 0.011 \\
\hline$G-M$ & -0.124 & 0.988 & 0.103 & 0.992 \\
\hline$J-L$ & -0.283 & 0.371 & 0.296 & 0.227 \\
\hline$J-M$ & 0.075 & 0.999 & -0.067 & 0.999 \\
\hline$L-M$ & 0.359 & 0.303 & -0.364 & 0.166 \\
\hline
\end{tabular}




\section{Faecal Glucocorticoid Metabolites}

Table S11 Influence of group size on mean monthly fGCM concentrations (In-transformed) in seven groups of Verreaux's sifakas; results of the full model (GLMM; Nobservations $\left.=729, N_{I D}=42\right)$.

\begin{tabular}{|c|c|c|c|c|c|c|c|c|c|}
\hline Term & Est & SE & Lower $\mathrm{Cl}$ & Upper $\mathrm{Cl}$ & $\operatorname{Min}^{a}$ & $\operatorname{Max}^{a}$ & $\chi^{2 b}$ & $d f$ & $P$ \\
\hline (Intercept) & -1.472 & 0.062 & -1.623 & -1.317 & -1.471 & -1.526 & c & c & c \\
\hline$\Delta T_{e m p}{ }^{d}$ & 0.354 & 0.037 & 0.243 & 0.459 & 0.354 & 0.332 & c & c & c \\
\hline Food availabilitye & 0.126 & 0.036 & 0.021 & 0.231 & 0.126 & 0.100 & c & c & c \\
\hline Group size ${ }^{f}$ & -0.024 & 0.060 & -0.169 & 0.122 & -0.024 & -0.092 & c & c & c \\
\hline Group size ${ }^{2 f}$ & 0.070 & 0.045 & -0.044 & 0.194 & 0.070 & 0.021 & c & c & c \\
\hline Yearg $^{g}$ & 0.007 & 0.017 & -0.042 & 0.062 & 0.007 & -0.007 & 0.179 & 1 & 0.672 \\
\hline Sex (males) & 0.308 & 0.073 & 0.135 & 0.472 & 0.307 & 0.272 & $12.352^{h}$ & $1^{\mathrm{h}}$ & $<0.001^{h}$ \\
\hline$\Delta$ Temp*Group size & 0.019 & 0.028 & -0.070 & 0.106 & 0.018 & -0.013 & 0.389 & 1 & 0.533 \\
\hline$\Delta$ Temp*Group size ${ }^{2}$ & -0.001 & 0.025 & -0.078 & 0.072 & 0.000 & -0.038 & 0.000 & 1 & 0.984 \\
\hline Food avail*Group size & 0.058 & 0.028 & -0.031 & 0.149 & 0.058 & 0.031 & 3.706 & 1 & 0.054 \\
\hline Food avail*Group size ${ }^{2}$ & -0.008 & 0.024 & -0.085 & 0.072 & -0.008 & -0.025 & 0.104 & 1 & 0.747 \\
\hline \multicolumn{10}{|c|}{$\begin{array}{l}\text { a Minimum and maximum of model estimates obtained when dropping levels of random effects one at a time } \\
\text { b Results of a likelihood ratio test comparing the full model with a reduced model lacking the respective term } \\
\text { c Not shown as not having a meaningful interpretation. See footnotes of Table S1 for details. } \\
\text { d z-transformed, mean and SD of the original values were } 16.449 \text { and } 4.264 \text {, respectively } \\
\text { e z-transformed, mean and SD of the original values were } 2.804 \text { and } 0.972 \text {, respectively } \\
{ }^{f} \text { z-transformed, mean and SD of the original values were } 6.475 \text { and } 2.155 \text {, respectively } \\
\text { z } \text { z-transformed, mean and SD of the original values were } 1.551 \text { and } 0.498 \text {, respectively } \\
\text { h Values refer to the overall test of the effect of the predictor ("Sex"), not the specific level indicated in the respective row }\end{array}$} \\
\hline
\end{tabular}

Table S12 Influence of group ID on mean monthly fGCM concentrations (In-transformed) in seven groups of Verreaux's sifakas; results of the full model (GLMM; $\left.N_{\text {Observations }}=729, N_{I D}=42\right)$.

\begin{tabular}{|c|c|c|c|c|c|c|c|c|c|c|}
\hline Term & & Est & SE & Lower Cl & Upper Cl & $\operatorname{Min}^{a}$ & $\operatorname{Max}^{a}$ & $\chi^{2 b}$ & $d f$ & $P$ \\
\hline \multicolumn{2}{|l|}{ (Intercept) } & -1.442 & 0.091 & -1.649 & -1.227 & -1.503 & -1.376 & $\bar{c}$ & $\mathrm{c}$ & c \\
\hline \multicolumn{2}{|l|}{$\Delta$ Temp $^{\mathrm{d}}$} & 0.357 & 0.026 & 0.280 & 0.433 & 0.333 & 0.369 & 26.061 & 1 & $<0.001$ \\
\hline \multicolumn{2}{|c|}{ Food availability } & 0.124 & 0.023 & 0.050 & 0.195 & 0.104 & 0.141 & 10.232 & 1 & 0.001 \\
\hline \multirow[t]{6}{*}{ Group } & $\mathrm{F}$ & -0.068 & 0.102 & -0.288 & 0.165 & -0.118 & -0.022 & $5.602^{f}$ & $6^{f}$ & $0.469^{f}$ \\
\hline & F1 & 0.001 & 0.141 & -0.322 & 0.322 & -0.186 & 0.185 & c & c & c \\
\hline & G & -0.118 & 0.119 & -0.416 & 0.153 & -0.204 & -0.037 & c & c & c \\
\hline & J & 0.042 & 0.110 & -0.216 & 0.300 & -0.013 & 0.109 & c & c & c \\
\hline & $\mathrm{L}$ & -0.016 & 0.117 & -0.280 & 0.255 & -0.079 & 0.061 & c & c & c \\
\hline & M & 0.154 & 0.123 & -0.131 & 0.440 & 0.096 & 0.222 & c & c & c \\
\hline \multicolumn{2}{|l|}{ Yearg } & 0.001 & 0.016 & -0.052 & 0.048 & -0.009 & 0.015 & 0.002 & 1 & 0.964 \\
\hline \multicolumn{2}{|l|}{ Sex (males) } & 0.332 & 0.066 & 0.177 & 0.479 & 0.309 & 0.354 & $19.655^{f}$ & $1^{f}$ & $<0.001^{f}$ \\
\hline \multicolumn{11}{|c|}{$\begin{array}{l}\text { a Minimum and maximum of model estimates obtained when dropping levels of random effects one at a time } \\
\text { b Results of a likelihood ratio test comparing the full model with a reduced model lacking the respective term } \\
\text { " Not shown as not having a meaningful interpretation. See footnotes of Table S1 for details. } \\
{ }^{d} \text { z-transformed, mean and SD of the original values were } 16.449 \text { and } 4.264 \text {, respectively } \\
\text { e z-transformed, mean and SD of the original values were } 2.804 \text { and } 0.972 \text {, respectively } \\
\text { f Values refer to the overall test of the effect of the predictor ("Group","Sex"), not the specific level indicated in the respective row } \\
\mathrm{g}_{\mathrm{Z}} \text {-transformed, mean and SD of the original values were } 1.551 \text { and } 0.498 \text {, respectively }\end{array}$} \\
\hline
\end{tabular}




\section{Food Tree Density and Species Richness}

Table S13 Results of post-hoc comparisons of groups' habitats in terms of average food tree densities and food tree species richness. Post-hoc comparisons, following a Kruskal Wallis test, of food tree densities were conducted with Dunn's pairwise post-hoc tests with Bonferroni correction. Species richness was compared with Tukey post-hoc tests, following a one-way ANOVA. Significant $p$-values are highlighted in bold.

\begin{tabular}{|c|c|c|c|c|}
\hline \multirow{2}{*}{ Groups } & \multicolumn{2}{|c|}{ Food tree density } & \multicolumn{2}{|c|}{ Food tree species richness } \\
\hline & $\mathrm{Z}$ & $p$ adj & Estimate & $p$ adj \\
\hline$E-F$ & -0.857 & 1.000 & -0.500 & 1.000 \\
\hline$E-F 1$ & -2.074 & 0.800 & -1.567 & 0.981 \\
\hline$E-G$ & -1.332 & 1.000 & 0.500 & 1.000 \\
\hline$E-J$ & 0.839 & 1.000 & -1.100 & 0.994 \\
\hline$E-L$ & 1.696 & 1.000 & -0.300 & 1.000 \\
\hline$E-M$ & 2.801 & 0.075 & 4.000 & 0.199 \\
\hline$F-F 1$ & 0.926 & 1.000 & -1.067 & 0.998 \\
\hline$F-G$ & 1.783 & 1.000 & 1.000 & 0.996 \\
\hline$F-J$ & 2.876 & 1.000 & -0.600 & 1.000 \\
\hline$F-L$ & 0.087 & 1.000 & 0.200 & 1.000 \\
\hline$F-M$ & 0.326 & 0.834 & 4.500 & 0.104 \\
\hline$F 1-G$ & 1.183 & 0.107 & 2.067 & 0.928 \\
\hline F1 - J & 2.357 & 0.084 & 0.467 & 1.000 \\
\hline $\mathrm{F} 1-\mathrm{L}$ & -0.513 & 0.387 & 1.267 & 0.994 \\
\hline $\mathrm{F} 1-\mathrm{M}$ & -0.600 & 1.000 & 5.567 & 0.065 \\
\hline$G-J$ & -2.913 & 1.000 & -1.600 & 0.957 \\
\hline$G-L$ & -2.057 & 1.000 & -0.800 & 0.999 \\
\hline$G-M$ & -0.449 & 0.004 & 3.500 & 0.345 \\
\hline$J-L$ & -3.753 & 1.000 & 0.800 & 0.999 \\
\hline$J-M$ & -3.840 & 0.003 & 5.100 & 0.042 \\
\hline$L-M$ & -3.240 & 0.025 & 4.300 & 0.136 \\
\hline
\end{tabular}


a)

b)

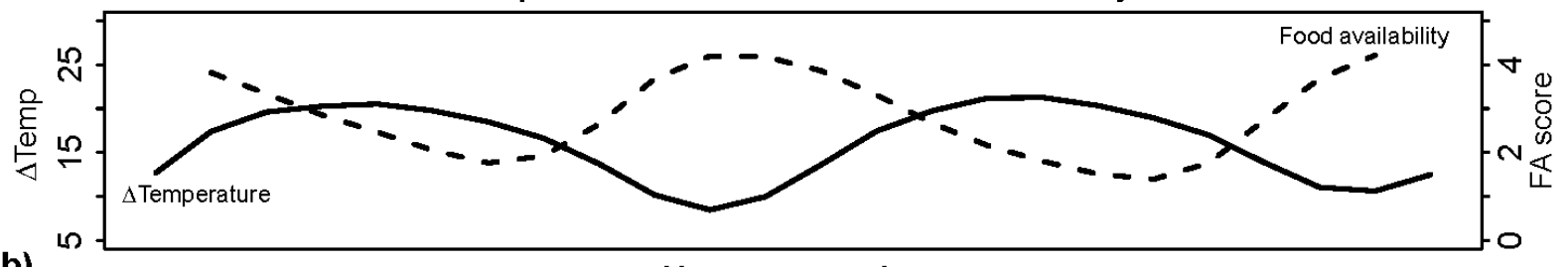

Home range size

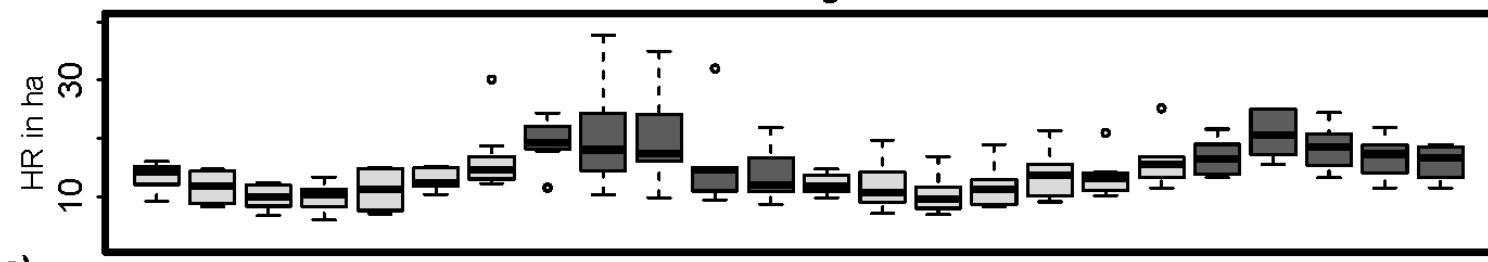

c)

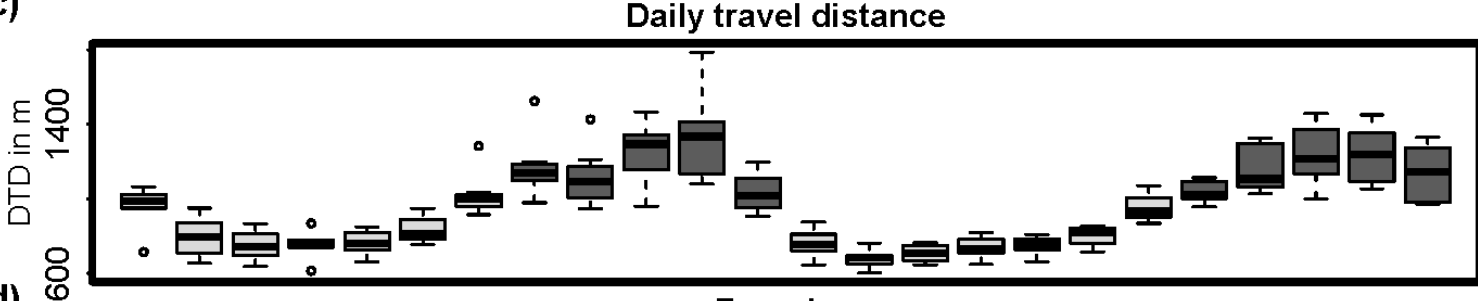

d)

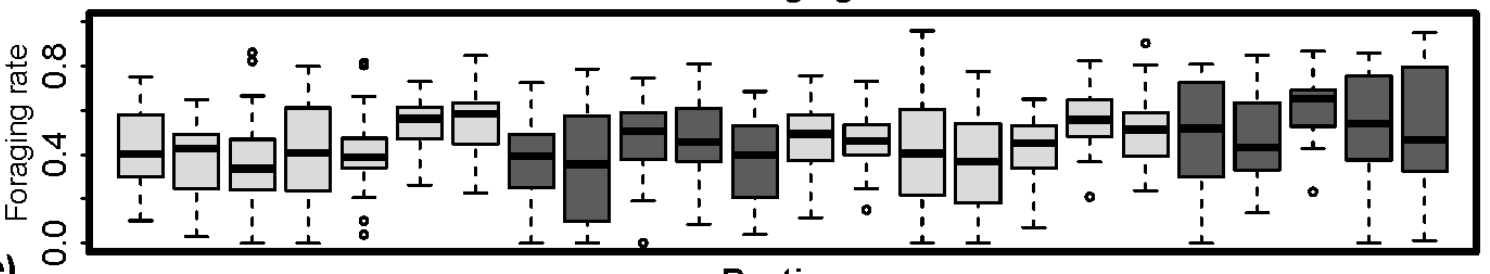

e)

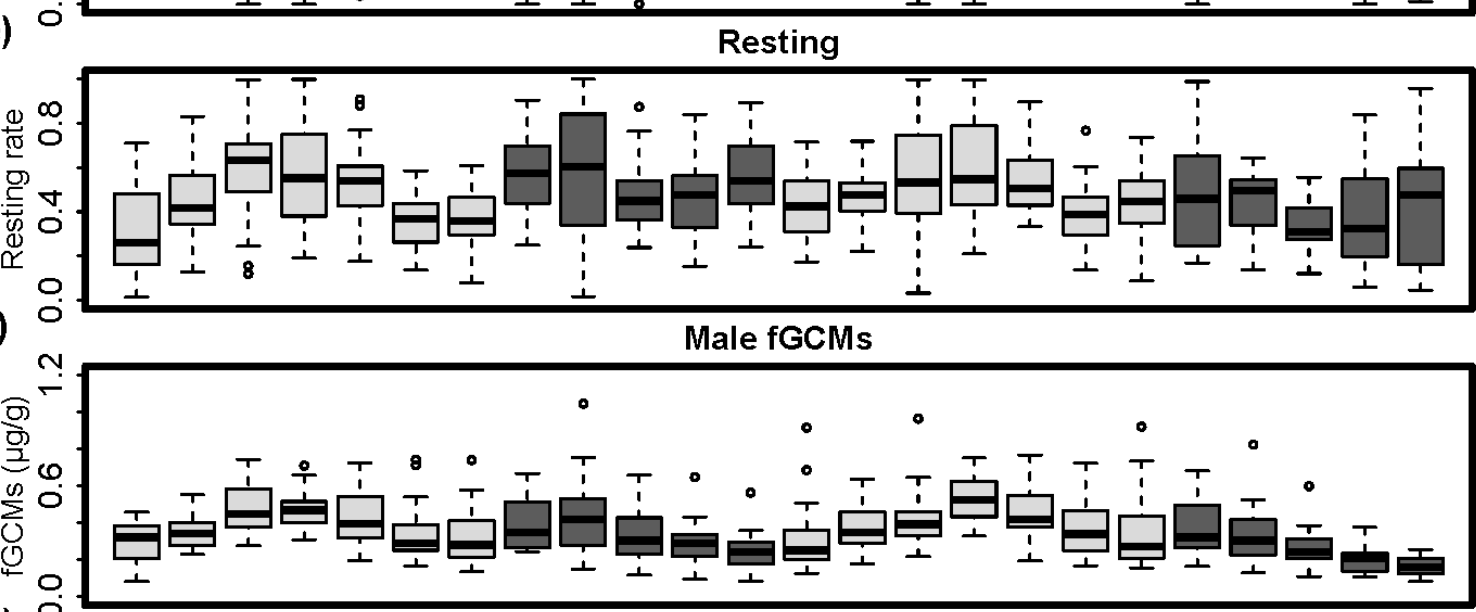

g)

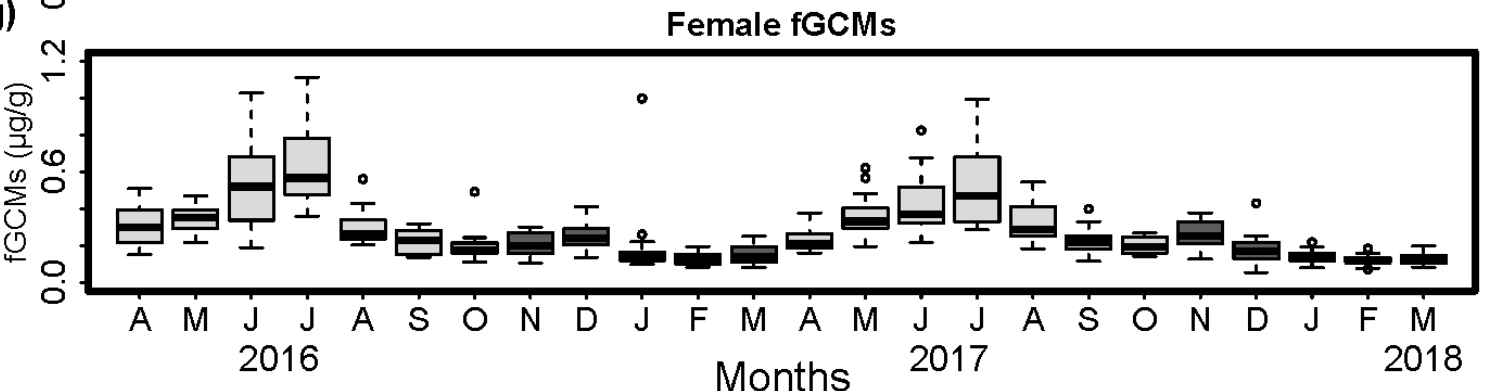

Figure S1 Effects of seasonal changes on different behavioural and physiological measures. Boxplots comprise data on mean monthly values for each group (home range size, daily travel distances) or individual (foraging, resting, fGCMs). Dark grey boxplots represent the wet season, light grey boxplots represent the dry season. Highlighted graphs indicate significant differences across the seasons. Boxplots indicate median, upper and lower quartiles. Whiskers indicate +/- 1.5 interquartile ranges and small circles bevond whiskers indicate outliers. 


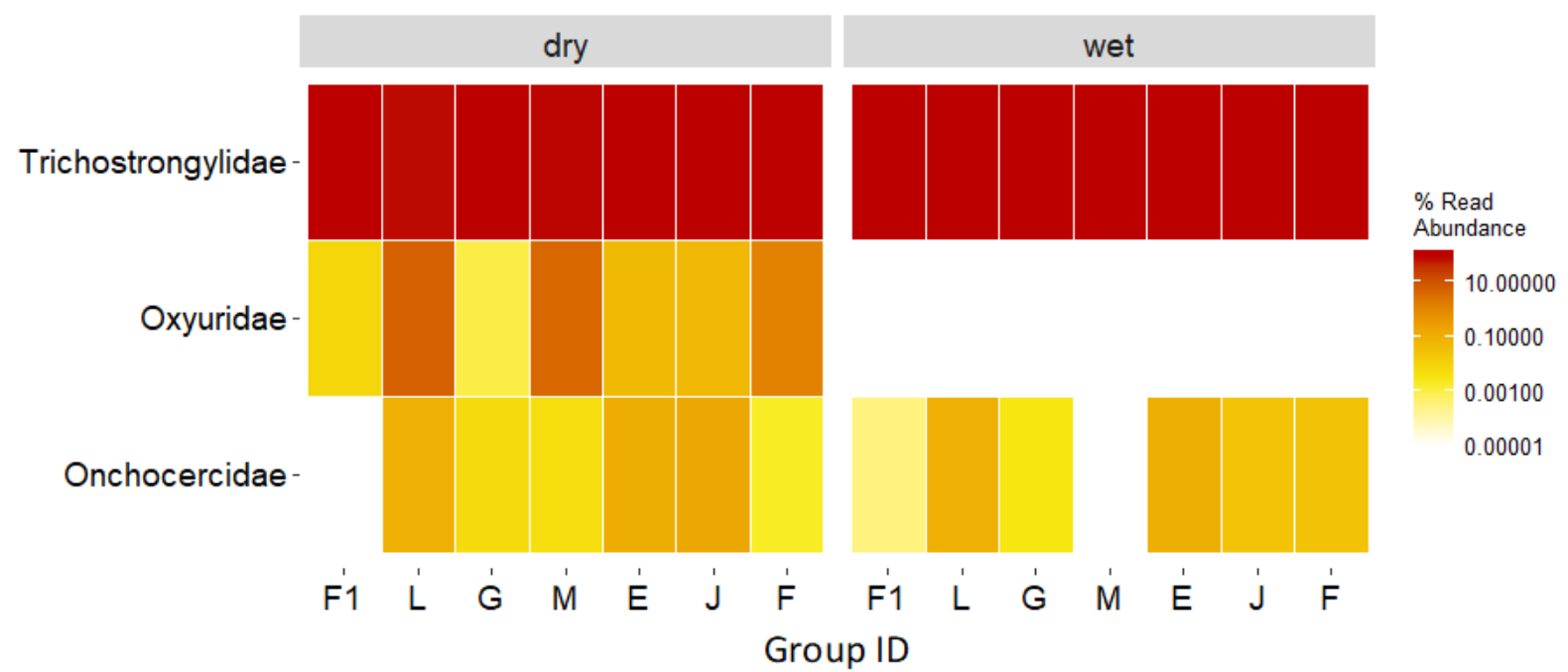

Figure S2 Abundance of three nematode families in seven neighbouring sifaka groups during dry and wet season. Data on intestinal helminth infestations were compiled with next-generation sequencing of $18 \mathrm{~S}$ rRNA genes ( $\mathrm{N}=487$ samples). 


\section{3) Further Analyses}

\section{Results of GLMMs with Group Size Measures, Excluding Dependent Infants (age $<9 \mathrm{~m}$ ):}

Altogether, we did not detect major differences in group size effects in any of the five models when using two different measures of group size - one including dependent infants (see Tables S1, S3, S6, S8 \& S11), and the other one excluding dependent infants (see Tables S14-S18).

\section{Ranging Patterns}

\section{Home Range Size}

The model was significant $\left(\chi^{2}=47.702, d f=9, p<0.001, R_{m / c}^{2}=0.30 / 0.78\right)$, however, group size was not correlated to home range sizes (Table S14).

Table S14 Influence of group size (without dependent infants) on home range size (In-transformed) in seven groups of Verreaux's sifakas; results of the full model $\left(G L M M ; N_{\text {Observations }}=143, N_{I D}=41\right)$.

\begin{tabular}{|c|c|c|c|c|c|c|c|c|c|}
\hline Term & Est & SE & Lower $\mathrm{Cl}$ & Upper Cl & $\operatorname{Min}^{a}$ & $\operatorname{Max}^{a}$ & $\chi^{2 b}$ & $d f$ & $P$ \\
\hline (Intercept) & 2.572 & 0.108 & 2.372 & 2.799 & 2.488 & 2.641 & c & $\mathrm{c}$ & c \\
\hline Year ${ }^{\mathrm{d}}$ & -0.025 & 0.031 & -0.086 & 0.035 & -0.045 & -0.005 & 0.629 & 1 & 0.428 \\
\hline$\Delta$ Temp $^{\mathrm{e}}$ & -0.273 & 0.031 & -0.335 & -0.215 & -0.303 & -0.235 & c & c & c \\
\hline Food availability ${ }^{f}$ & -0.209 & 0.038 & -0.281 & -0.141 & -0.231 & -0.175 & c & c & c \\
\hline Group size ${ }^{g}$ & -0.094 & 0.088 & -0.29 & 0.097 & -0.186 & -0.043 & c & c & c \\
\hline Group size $2 \mathrm{~g}$ & 0.078 & 0.044 & -0.025 & 0.172 & -0.005 & 0.103 & c & c & c \\
\hline$\Delta$ Temp*Group size & -0.028 & 0.024 & -0.076 & 0.019 & -0.046 & 0.113 & 1.368 & 1 & 0.242 \\
\hline$\Delta$ Temp*Group size $^{2}$ & 0.019 & 0.019 & -0.017 & 0.055 & -0.059 & 0.132 & 0.965 & 1 & 0.326 \\
\hline Food avail*Group size & 0.036 & 0.032 & -0.028 & 0.095 & 0.020 & 0.183 & 1.247 & 1 & 0.264 \\
\hline Food avail*Group size ${ }^{2}$ & 0.000 & 0.023 & -0.046 & 0.048 & -0.069 & 0.106 & 0.000 & 1 & 0.984 \\
\hline \multicolumn{10}{|c|}{$\begin{array}{l}\text { a Minimum and maximum of model estimates obtained when dropping levels of random e } \\
\text { b Results of a likelihood ratio test comparing the full model with a reduced model lacking th } \\
\text { c Not shown as not having a meaningful interpretation. See footnotes of Table S1 for detail } \\
{ }^{d} \text { z-transformed, mean and SD of the original values were } 1.503 \text { and } 0.502 \text {, respectively } \\
\text { e z-transformed, mean and SD of the original values were } 16.398 \text { and } 4.269 \text {, respectively } \\
{ }^{f} \text {-transformed, mean and SD of the original values were } 2.811 \text { and } 0.967 \text {, respectively } \\
{ }^{\mathrm{z}} \text {-transformed, mean and SD of the original values were } 5.084 \text { and } 1.878 \text {, respectively }\end{array}$} \\
\hline
\end{tabular}

Daily Travel Distances

The model was significant $\left(\chi^{2}=65.088, d f=9, p<0.001, R_{m / c}^{2}=0.67 / 0.83\right)$. There was an interaction effect with DTDs and $\Delta$ temperatures, i.e. during months with increased $\Delta$ temperatures, during the months of the dry season, larger groups had shorter DTDs than smaller groups (test of the interaction between $\Delta$ temperatures and group size: $\chi^{2}=10.229, d f=1, p=0.001$ ) (Table S15). 
Table S15 Influence of group size (without dependent infants) on daily travel distance (In-transformed) in seven groups of Verreaux's sifakas; results of the full model $\left(G L M M ; N_{\text {observations }}=143, N_{I D}=41\right)$.

\begin{tabular}{|c|c|c|c|c|c|c|c|c|c|}
\hline Term & Est & SE & Lower $\mathrm{Cl}$ & Upper Cl & $\operatorname{Min}^{a}$ & $\operatorname{Max}^{a}$ & $\chi^{2 b}$ & $d f$ & $P$ \\
\hline (Intercept) & 6.819 & 0.027 & 6.759 & 6.875 & 6.790 & 6.851 & c & $\mathrm{c}$ & c \\
\hline Year $^{d}$ & -0.043 & 0.018 & -0.078 & -0.006 & -0.056 & -0.029 & 4.039 & 1 & 0.044 \\
\hline$\Delta$ Temp $^{\mathrm{e}}$ & -0.278 & 0.017 & -0.312 & -0.245 & -0.298 & -0.261 & c & c & c \\
\hline Food availability ${ }^{f}$ & -0.153 & 0.017 & -0.186 & -0.120 & -0.162 & -0.139 & c & c & c \\
\hline Group sizeg & -0.039 & 0.042 & -0.132 & 0.053 & -0.134 & -0.001 & c & c & c \\
\hline Group size $\mathrm{e}^{2 \mathrm{~g}}$ & 0.016 & 0.030 & -0.054 & 0.084 & -0.105 & 0.068 & c & c & c \\
\hline$\Delta$ Temp*Group size & -0.043 & 0.013 & -0.068 & -0.019 & -0.052 & 0.026 & 10.229 & 1 & 0.001 \\
\hline$\Delta$ Temp*Group size ${ }^{2}$ & 0.018 & 0.011 & -0.004 & 0.038 & -0.016 & 0.077 & 2.685 & 1 & 0.101 \\
\hline Food avail*Group size & -0.025 & 0.014 & -0.052 & 0.000 & -0.029 & 0.012 & 3.276 & 1 & 0.070 \\
\hline Food avail*Group size ${ }^{2}$ & 0.009 & 0.011 & -0.013 & 0.029 & -0.009 & 0.038 & 0.669 & 1 & 0.413 \\
\hline \multicolumn{10}{|c|}{$\begin{array}{l}\text { a Minimum and maximum of model estimates obtained when dropping levels of random } \\
\text { b Results of a likelihood ratio test comparing the full model with a reduced model lacking } \\
\text { c Not shown as not having a meaningful interpretation. See footnotes of Table S1 for deta } \\
\text { d z-transformed, mean and SD of the original values were } 1.503 \text { and } 0.502 \text {, respectively } \\
\text { e-transformed, mean and SD of the original values were } 16.398 \text { and } 4.269 \text {, respectively } \\
{ }^{f} z \text {-transformed, mean and SD of the original values were } 2.811 \text { and } 0.967 \text {, respectively } \\
{ }^{g} \text { z-transformed, mean and SD of the original values were } 5.084 \text { and } 1.878 \text {, respectively }\end{array}$} \\
\hline
\end{tabular}

\section{Daily Activities}

\section{Foraging}

The model was significant $\left(\chi^{2}=13.865, d f=5, p=0.0 .016\right)$, however, group size was not correlated to foraging rates (Table S16).

Table S16 Influence of group size (without dependent infants) on foraging rates in seven groups of Verreaux's sifakas; results of the full model (GLMM; $\left.N_{\text {Observations }}=659, N_{I D}=41\right)$.

\begin{tabular}{|c|c|c|c|c|c|c|c|c|c|}
\hline Term & Est & SE & Lower $\mathrm{Cl}$ & Upper Cl & $\operatorname{Min}^{a}$ & $\operatorname{Max}^{a}$ & $\chi^{2 b}$ & $d f$ & $P$ \\
\hline (Intercept) & -0.270 & 0.042 & -0.352 & -0.188 & -0.281 & -0.251 & c & $\mathrm{c}$ & c \\
\hline Year $^{d}$ & 0.096 & 0.039 & 0.019 & 0.173 & 0.080 & 0.109 & 5.823 & 1 & 0.016 \\
\hline$\Delta$ Temp $^{\mathrm{e}}$ & -0.168 & 0.056 & -0.279 & -0.058 & -0.207 & -0.141 & c & c & c \\
\hline Food availability ${ }^{f}$ & -0.089 & 0.051 & -0.188 & 0.010 & -0.134 & -0.067 & c & c & c \\
\hline Group size ${ }^{g}$ & 0.103 & 0.047 & 0.010 & 0.195 & 0.079 & 0.125 & c & c & c \\
\hline$\Delta$ Temp*Group size & 0.065 & 0.056 & -0.046 & 0.175 & 0.013 & 0.103 & 1.304 & 1 & 0.253 \\
\hline Food avail*Group size & 0.054 & 0.051 & -0.045 & 0.154 & 0.011 & 0.077 & 1.139 & 1 & 0.286 \\
\hline \multicolumn{10}{|c|}{$\begin{array}{l}\text { a Minimum and maximum of model estimates obtained when dropping levels of random } \\
\text { b Results of a likelihood ratio test comparing the full model with a reduced model lacking } \\
\text { " Not shown as not having a meaningful interpretation. See footnotes of Table S1 for deta } \\
{ }^{d} z \text {-transformed, mean and SD of the original values were } 1.525 \text { and } 0.500 \text {, respectively } \\
\text { e z-transformed, mean and SD of the original values were } 16.687 \text { and } 4.278 \text {, respectively } \\
\text { fz-transformed, mean and SD of the original values were } 2.803 \text { and } 0.973 \text {, respectively } \\
{ }^{\mathrm{g}} \text { z-transformed, mean and SD of the original values were } 5.781 \text { and } 1.934 \text {, respectively }\end{array}$} \\
\hline
\end{tabular}




\section{Resting}

The model was significant $\left(\chi^{2}=15.440, d f=5, p=0.009\right)$, however, group size was not correlated to resting rates (Table S17).

Table S17 Influence of group size (without dependent infants) on resting rates in seven groups of Verreaux's sifakas; results of the full model (GLMM; Nobservations=659, $\left.N_{I D}=41\right)$.

\begin{tabular}{|c|c|c|c|c|c|c|c|c|c|}
\hline Term & Est & SE & Lower $\mathrm{Cl}$ & Upper Cl & $\operatorname{Min}^{a}$ & $\operatorname{Max}^{a}$ & $\chi^{2 b}$ & $d f$ & $P$ \\
\hline (Intercept) & -0.023 & 0.039 & -0.099 & 0.053 & -0.042 & -0.011 & c & c & c \\
\hline Year ${ }^{\mathrm{d}}$ & -0.067 & 0.034 & -0.134 & 0.000 & -0.080 & -0.055 & 3.870 & 1 & 0.049 \\
\hline$\Delta T_{e m p}{ }^{e}$ & 0.152 & 0.049 & 0.056 & 0.247 & 0.131 & 0.185 & c & c & c \\
\hline Food availability ${ }^{f}$ & 0.046 & 0.048 & -0.047 & 0.139 & 0.029 & 0.082 & c & c & c \\
\hline Group size $\mathrm{g}^{\mathrm{g}}$ & -0.093 & 0.044 & -0.180 & -0.006 & -0.116 & -0.071 & c & c & c \\
\hline$\Delta$ Temp*Group size & -0.025 & 0.050 & -0.122 & 0.073 & -0.049 & 0.016 & 0.243 & 1 & 0.622 \\
\hline Food avail*Group size & -0.043 & 0.048 & -0.137 & 0.051 & -0.064 & -0.009 & 0.803 & 1 & 0.370 \\
\hline \multicolumn{10}{|c|}{ 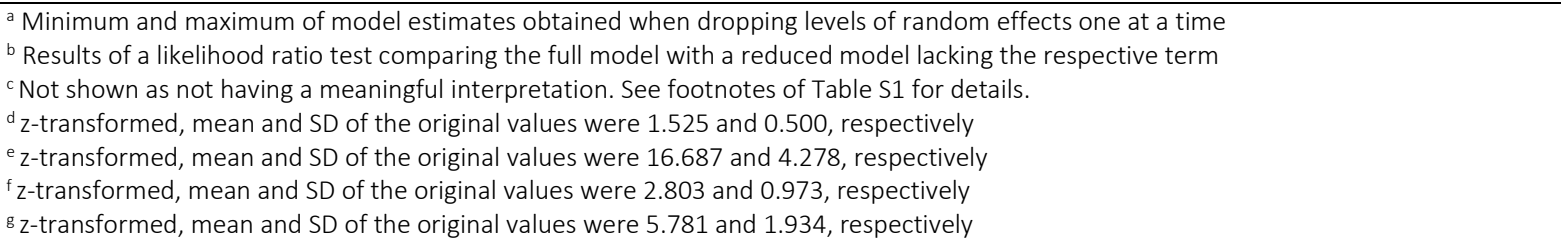 } \\
\hline
\end{tabular}




\section{Faecal Glucocorticoid Metabolites}

The model was significant ( $\left.\chi^{2}=56.505, d f=10, p<0.001, R_{m / c}^{2}=0.26 / 0.50\right)$.

Table S18 Influence of group size (without dependent infants) on mean monthly fGCM concentrations (In-transformed) in seven groups of Verreaux's sifakas; results of the full model (GLMM; $\left.N_{\text {observations }}=729, N_{I D}=42\right)$.

\begin{tabular}{|c|c|c|c|c|c|c|c|c|c|}
\hline Term & Est & SE & Lower $\mathrm{Cl}$ & Upper $\mathrm{Cl}$ & $\operatorname{Min}^{a}$ & $\operatorname{Max}^{a}$ & $\chi^{2 b}$ & $d f$ & $P$ \\
\hline (Intercept) & -1.471 & 0.058 & -1.610 & -1.325 & -1.483 & -1.431 & c & c & c \\
\hline$\Delta$ Temp $^{\mathrm{d}}$ & 0.425 & 0.057 & 0.285 & 0.576 & 0.360 & 0.506 & c & c & c \\
\hline Food availability & 0.128 & 0.033 & 0.029 & 0.226 & 0.102 & 0.151 & c & c & c \\
\hline Group size ${ }^{f}$ & 0.083 & 0.066 & -0.131 & 0.284 & -0.027 & 0.104 & c & c & c \\
\hline Group size $e^{2 f}$ & 0.127 & 0.093 & -0.091 & 0.361 & -0.004 & 0.177 & c & c & c \\
\hline Yearg & -0.001 & 0.018 & -0.057 & 0.055 & -0.011 & 0.009 & 0.005 & 1 & 0.941 \\
\hline Sex (males) & 0.328 & 0.067 & 0.173 & 0.476 & 0.305 & 0.348 & $17.644^{h}$ & $1^{\mathrm{h}}$ & $<0.001^{\mathrm{h}}$ \\
\hline$\Delta$ Temp*Group size & 0.096 & 0.041 & -0.019 & 0.221 & 0.014 & 0.245 & 2.505 & 1 & 0.113 \\
\hline$\Delta$ Temp $^{*}$ Group size ${ }^{2}$ & -0.031 & 0.028 & -0.121 & 0.058 & -0.047 & 0.014 & 1.191 & 1 & 0.275 \\
\hline Food avail*Group size & 0.011 & 0.025 & -0.076 & 0.091 & -0.003 & 0.209 & 0.174 & 1 & 0.677 \\
\hline Food avail*Group size ${ }^{2}$ & -0.003 & 0.022 & -0.071 & 0.067 & -0.023 & 0.127 & 0.052 & 1 & 0.819 \\
\hline \multicolumn{10}{|c|}{$\begin{array}{l}\text { a Minimum and maximum of model estimates obtained when dropping levels of random effects one at a time } \\
\text { b Results of a likelihood ratio test comparing the full model with a reduced model lacking the respective term } \\
\text { c Not shown as not having a meaningful interpretation. See footnotes of Table S1 for details. } \\
\text { d z-transformed, mean and SD of the original values were } 16.449 \text { and } 4.264 \text {, respectively } \\
\text { e z-transformed, mean and SD of the original values were } 2.804 \text { and } 0.972 \text {, respectively } \\
\text { f z-transformed, mean and SD of the original values were } 5.739 \text { and } 1.919 \text {, respectively } \\
\text { g z-transformed, mean and SD of the original values were } 1.551 \text { and } 0.498 \text {, respectively } \\
\text { h Values refer to the overall test of the effect of the predictor ("Sex"), not the specific level indicated in the respective row }\end{array}$} \\
\hline
\end{tabular}




\section{Results of Correlations between Core Areas and Group Sizes or Group IDs}

Mean monthly core area sizes (50\% Kernels) and home range sizes (95\% Kernels) of all groups were highly correlated in Verreaux's sifakas (Pearson: $r=0.987, n=22, p<0.001$ ), which is why we only considered home range sizes in our main analyses. Here, we report the analyses and results of correlation between group size or group ID with core area sizes.

We applied GLMMs in the same way as described above for the models on home range sizes. We In-transformed the response (core areas) and used group ID as random effect. Z-transformed group size and group size-squared or group ID, food availability, temperature differences and study year comprised predictor variables and random slopes within group ID.

Group Size (incl. Dependent Infants) vs. Core Areas

Core areas in Verreaux's sifakas' home ranges had monthly average sizes of $4.01 \pm 1.96$ ha. The model examining effects of group size, food availability, temperature differences and study year on core area sizes was significant $\left(\chi^{2}=50.550, d f=9, p<0.001, R_{m / c}^{2}=0.44 / 0.69\right)$, however, group size was not correlated to core area sizes (Table S19, Fig. S3a).

Table S19 Influence of group size on core area sizes (In-transformed) in seven groups of Verreaux's sifakas; results of the full model $\left(\right.$ GLMM; Nobservations $\left.=143, N_{I D}=41\right)$.

\begin{tabular}{|c|c|c|c|c|c|c|c|c|c|}
\hline Term & Est & SE & Lower $\mathrm{Cl}$ & Upper Cl & $\operatorname{Min}^{a}$ & $\operatorname{Max}^{a}$ & $\chi^{2 b}$ & $d f$ & $P$ \\
\hline (Intercept) & 1.322 & 0.098 & 1.124 & 1.517 & 1.230 & 1.400 & c & c & c \\
\hline Year $^{d}$ & -0.015 & 0.028 & -0.073 & 0.044 & -0.029 & 0.008 & 0.283 & 1 & 0.595 \\
\hline$\Delta$ Temp $^{\mathrm{e}}$ & -0.417 & 0.044 & -0.508 & -0.327 & -0.448 & -0.399 & c & c & c \\
\hline Food availability ${ }^{f}$ & -0.272 & 0.043 & -0.356 & -0.178 & -0.316 & -0.243 & c & c & c \\
\hline Group size ${ }^{\mathrm{g}}$ & 0.004 & 0.074 & -0.158 & 0.162 & -0.097 & 0.060 & c & c & c \\
\hline Group size $2 \mathrm{~g}$ & -0.037 & 0.046 & -0.138 & 0.064 & -0.206 & -0.006 & c & c & c \\
\hline$\Delta$ Temp*Group size & -0.054 & 0.039 & -0.132 & 0.022 & -0.081 & 0.013 & 1.780 & 1 & 0.182 \\
\hline$\Delta$ Temp*Group size ${ }^{2}$ & -0.010 & 0.027 & -0.061 & 0.042 & -0.038 & 0.087 & 0.146 & 1 & 0.702 \\
\hline Food avail*Group size & -0.016 & 0.040 & -0.101 & 0.068 & -0.040 & 0.049 & 0.159 & 1 & 0.690 \\
\hline Food avail*Group size ${ }^{2}$ & -0.023 & 0.027 & -0.077 & 0.027 & -0.034 & 0.071 & 0.682 & 1 & 0.409 \\
\hline \multicolumn{10}{|c|}{$\begin{array}{l}\text { a Minimum and maximum of model estimates obtained when dropping levels of random } \\
\text { b Results of a likelihood ratio test comparing the full model with a reduced model lacking } \\
\text { c Not shown as not having a meaningful interpretation. See footnotes of Table S1 for deta } \\
\text { d z-transformed, mean and SD of the original values were } 1.503 \text { and } 0.502 \text {, respectively } \\
\text { ez-transformed, mean and SD of the original values were } 16.398 \text { and } 4.269 \text {, respectively } \\
{ }^{f} z \text {-transformed, mean and SD of the original values were } 2.811 \text { and } 0.967 \text {, respectively } \\
g_{z} \text {-transformed, mean and SD of the original values were } 5.790 \text { and } 2.072 \text {, respectively }\end{array}$} \\
\hline
\end{tabular}


Group Size (excl. Dependent Infants) vs. Core Areas

The model examining effects of group size (without dependent infants), food availability, temperature differences and study year on core area sizes was significant $\left(\chi^{2}=54.238, d f=9, p<0.001, R^{2} \mathrm{~m} / \mathrm{c}=\right.$ 0.44/0.71), however, group size was not correlated to core area sizes (Table S20).

Table S20 Influence of group size (without dependent infants) on core area sizes (In-transformed) in seven groups of Verreaux's sifakas; results of the full model $\left(G L M M ; N_{\text {observations }}=143, N_{I D}=41\right)$.

\begin{tabular}{|c|c|c|c|c|c|c|c|c|c|}
\hline Term & Est & SE & Lower $\mathrm{Cl}$ & Upper Cl & $\operatorname{Min}^{a}$ & $\operatorname{Max}^{a}$ & $\chi^{2 b}$ & $d f$ & $P$ \\
\hline (Intercept) & 1.230 & 0.100 & 1.044 & 1.429 & 1.167 & 1.292 & c & c & c \\
\hline Year ${ }^{d}$ & 0.000 & 0.028 & -0.059 & 0.056 & -0.016 & 0.025 & 0.000 & 1 & 0.999 \\
\hline$\Delta$ Temp $^{\mathrm{e}}$ & -0.411 & 0.041 & -0.490 & -0.334 & -0.440 & -0.369 & c & c & c \\
\hline Food availability ${ }^{f}$ & -0.262 & 0.040 & -0.341 & -0.179 & -0.311 & -0.235 & c & c & c \\
\hline Group sizeg & -0.074 & 0.087 & -0.261 & 0.108 & -0.181 & -0.036 & c & c & c \\
\hline Group size $2 \mathrm{~g}$ & 0.065 & 0.038 & -0.026 & 0.153 & 0.033 & 0.076 & c & c & c \\
\hline$\Delta$ Temp*Group size & -0.023 & 0.032 & -0.089 & 0.038 & -0.036 & 0.127 & 0.518 & 1 & 0.472 \\
\hline$\Delta$ Temp*Group size ${ }^{2}$ & -0.015 & 0.026 & -0.066 & 0.039 & -0.112 & 0.107 & 0.318 & 1 & 0.573 \\
\hline Food avail*Group size & 0.032 & 0.033 & -0.033 & 0.094 & 0.015 & 0.175 & 0.927 & 1 & 0.336 \\
\hline Food avail*Group size ${ }^{2}$ & -0.030 & 0.026 & -0.079 & 0.024 & -0.105 & 0.077 & 1.299 & 1 & 0.254 \\
\hline \multicolumn{10}{|c|}{$\begin{array}{l}\text { a Minimum and maximum of model estimates obtained when dropping levels of random ef } \\
\text { b Results of a likelihood ratio test comparing the full model with a reduced model lacking th } \\
\text { c Not shown as not having a meaningful interpretation. See footnotes of Table S1 for detail } \\
{ }^{d} \text { z-transformed, mean and SD of the original values were } 1.503 \text { and } 0.502 \text {, respectively } \\
\text { e z-transformed, mean and SD of the original values were } 16.398 \text { and } 4.269 \text {, respectively } \\
{ }^{f} \text {-transformed, mean and SD of the original values were } 2.811 \text { and } 0.967 \text {, respectively } \\
{ }_{\text {z }} \text {-transformed, mean and SD of the original values were } 5.084 \text { and } 1.878 \text {, respectively }\end{array}$} \\
\hline
\end{tabular}

\section{Group ID vs. Core Areas (50\% Kernels)}

The model examining effects of group ID, food availability, temperature differences and study year on core area sizes was significant $\left(\chi^{2}=74.139, d f=9, p<0.001, R_{m / c}^{2}=0.70 / 0.71\right)$, and groups differed significantly in their core area sizes (Table S21, Fig. S3b). More precisely, Groups M and L had larger core areas compared to all other groups (Table S5). 
Table S21 Influence of group size on core area sizes (In-transformed) in seven groups of Verreaux's sifakas; results of the full model $\left(G L M M\right.$; Nobservations $\left.=143, N_{I D}=41\right)$.

\begin{tabular}{|c|c|c|c|c|c|c|c|c|c|c|}
\hline Term & & Est & SE & Lower $\mathrm{Cl}$ & Upper Cl & $\operatorname{Min}^{a}$ & $\operatorname{Max}^{a}$ & $\chi^{2 b}$ & $d f$ & $P$ \\
\hline (Intercept) & & 1.225 & 0.053 & 1.123 & 1.328 & 1.195 & 1.225 & c & c & c \\
\hline \multirow[t]{6}{*}{ Group } & $\mathrm{F}$ & -0.030 & 0.075 & -0.191 & 0.117 & -0.030 & -0.030 & $27.033^{d}$ & $6^{d}$ & $<0.001^{d}$ \\
\hline & $\mathrm{F} 1$ & -0.064 & 0.075 & -0.219 & 0.086 & -0.064 & -0.034 & c & c & c \\
\hline & G & -0.091 & 0.075 & -0.245 & 0.044 & -0.091 & -0.061 & c & c & c \\
\hline & J & -0.216 & 0.081 & -0.373 & -0.069 & -0.224 & -0.182 & c & c & c \\
\hline & $L$ & 0.395 & 0.076 & 0.245 & 0.541 & 0.395 & 0.424 & c & c & c \\
\hline & $\mathrm{M}$ & 0.452 & 0.082 & 0.285 & 0.606 & 0.442 & 0.482 & c & c & c \\
\hline$\Delta T_{e m p}{ }^{e}$ & & -0.424 & 0.031 & -0.479 & -0.364 & -0.439 & -0.405 & 29.494 & 1 & $<0.001$ \\
\hline Food availability & & -0.288 & 0.032 & -0.349 & -0.226 & -0.318 & -0.270 & 21.705 & 1 & $<0.001$ \\
\hline Year' $^{\mathrm{g}}$ & & -0.015 & 0.024 & -0.061 & 0.033 & -0.029 & 0.005 & 0.371 & 1 & 0.542 \\
\hline 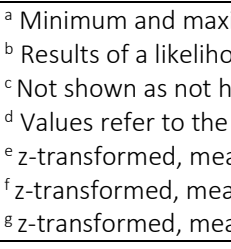 & $\begin{array}{l}\text { imum } \\
\text { od ra } \\
\text { laving } \\
\text { over } \\
\text { an an } \\
\text { an anc } \\
\text { an anc }\end{array}$ & $\begin{array}{l}f \text { model es } \\
\text { test comp } \\
\text { meaningfu } \\
\text { test of the } \\
\text { SD of the o } \\
\text { D of the or } \\
\text { SD of the o }\end{array}$ & $\begin{array}{l}\text { mates obt } \\
\text { ring the } f \\
\text { interpreta } \\
\text { ffect of th } \\
\text { sinal value } \\
\text { sinal value } \\
\text { sinal value }\end{array}$ & $\begin{array}{l}\text { ned when dro } \\
\text { I model with a } \\
\text { on. See footnc } \\
\text { predictor ("Gr } \\
\text { were } 16.398 \\
\text { were } 2.811 \text { an } \\
\text { were } 1.503 \text { ar } \\
\end{array}$ & $\begin{array}{l}\text { ping levels of } \\
\text { educed mode } \\
\text { es of Table S1 } \\
\text { oup"), not the } \\
\text { d 4.269, resp } \\
0.967 \text {, respec } \\
0.502 \text {, respe }\end{array}$ & $\begin{array}{l}\text { ndom effe } \\
\text { acking the } \\
\text { or details. } \\
\text { eecific level } \\
\text { tively } \\
\text { ively } \\
\text { ively }\end{array}$ & $\begin{array}{l}\text { sone at a t } \\
\text { spective te } \\
\text { dicated in }\end{array}$ & $\begin{array}{l}\text { me } \\
\text { he respective } \\
\text { he }\end{array}$ & row & \\
\hline
\end{tabular}

a)

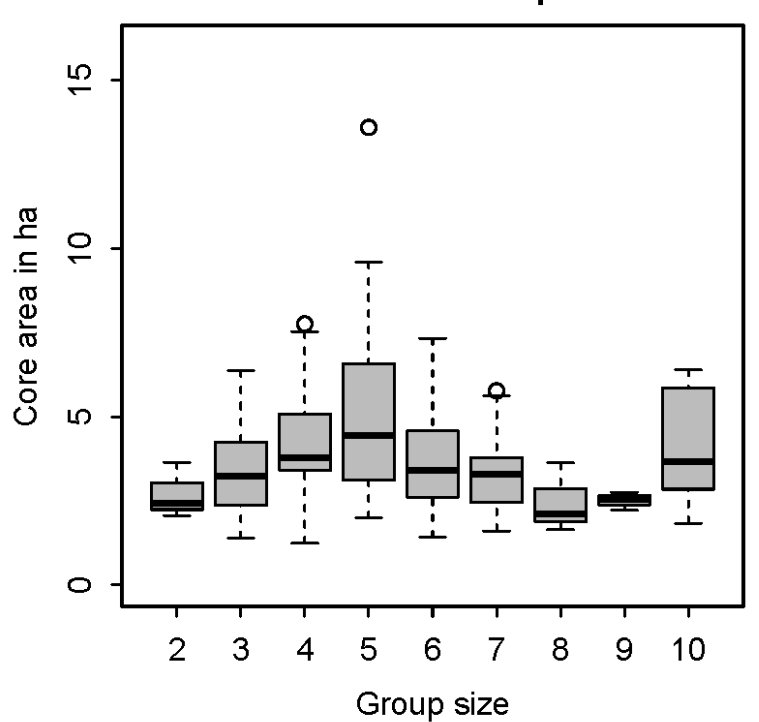

b) Core areas vs Group ID

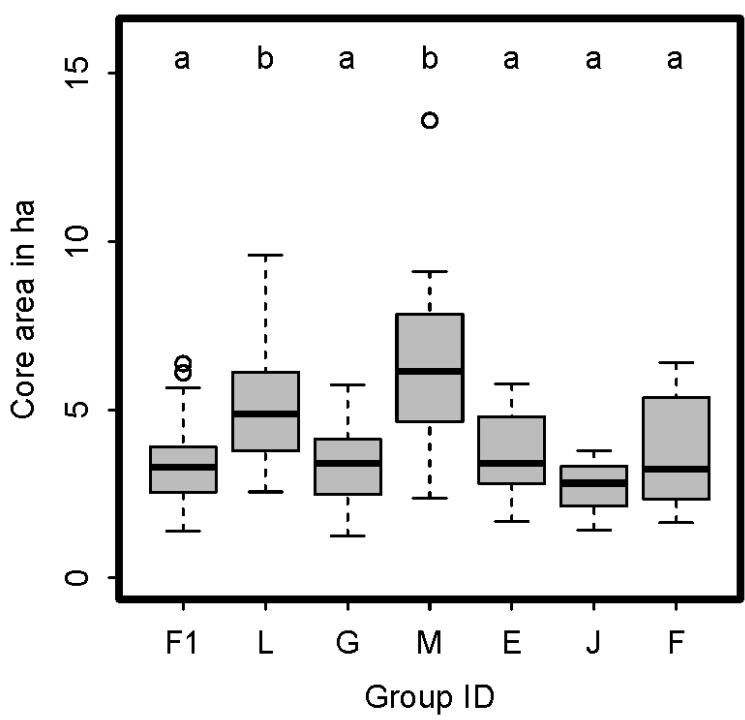

Figure S3 Group size and group ID versus core area sizes. Boxplots comprise data on mean monthly rates per group and indicate median, upper and lower quartiles. Whiskers indicate +/- 1.5 interquartile ranges and small circles beyond whiskers indicate outliers. Different letters indicate significant differences in means, i.e. groups sharing at least one letter do not differ. Highlighted graphs indicate significant differences among groups or group sizes. 


\section{$\underline{\text { Results of Correlations among FGCMs, Activity and Ranging Patterns }}$}

Here, we examined potential links among the behavioural and physiological measures we collected to study effects of group size variation on Verreaux's sifakas. Of these measures, fGCMs probably represent the best indicators for individual condition. FGCMs can be directly or indirectly affected by both, behavioural (foraging, resting or ranging) and ecological (temperature differences, food availability) variables (Dunn et al., 2013; Emery Thompson, 2017). We, therefore, investigated potential coherences among these variables to enhance the general understanding of how, if at all, these factors are related to each other in Verreaux's sifakas.

We applied generalized linear mixed-effect models (GLMMs) in the same manner as described above (see methods), to test whether individual mean monthly fGCMs (response variable) are correlated to monthly daily travel distances (DTD), monthly home range sizes (HR), monthly resting and foraging rates. We ran two models, the first one including DTD and foraging rates, the second one including HR and resting rates as predictors. We had to separate monthly measures of DTD and HR, and foraging and resting rates, as these variables are highly correlated with each other (Pearson: HR/DTD: $r=0.86, n=22$, $p<0.001$; foraging/resting: $r-0.91, p<0.001$, Figure $S 4 a \& b$ ), which would cause issues with collinearity

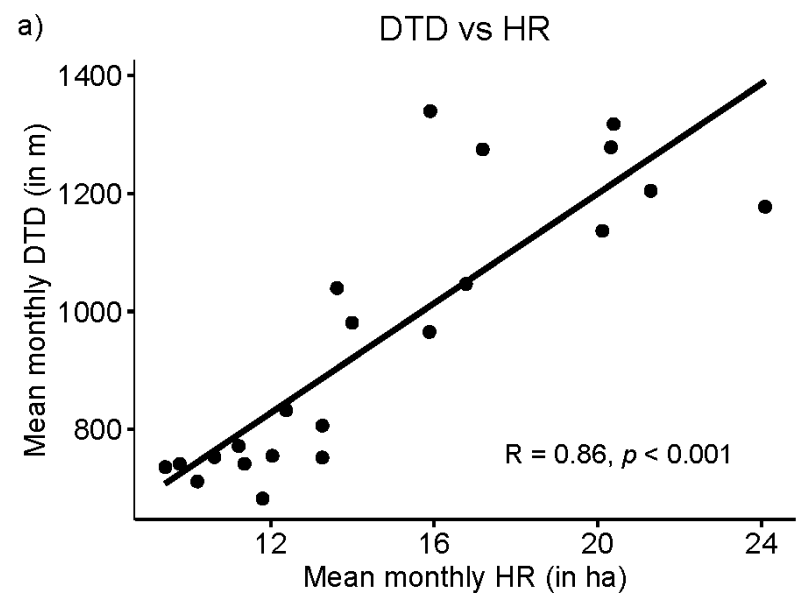

b) Foraging vs resting rate

c)

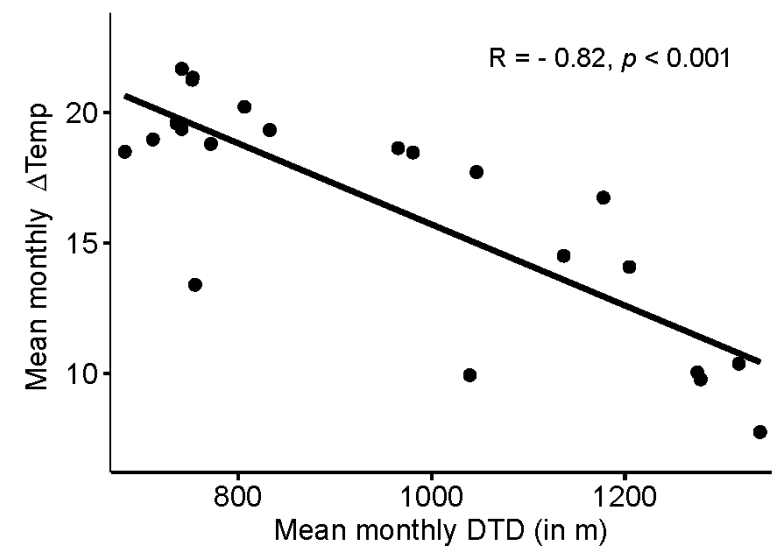

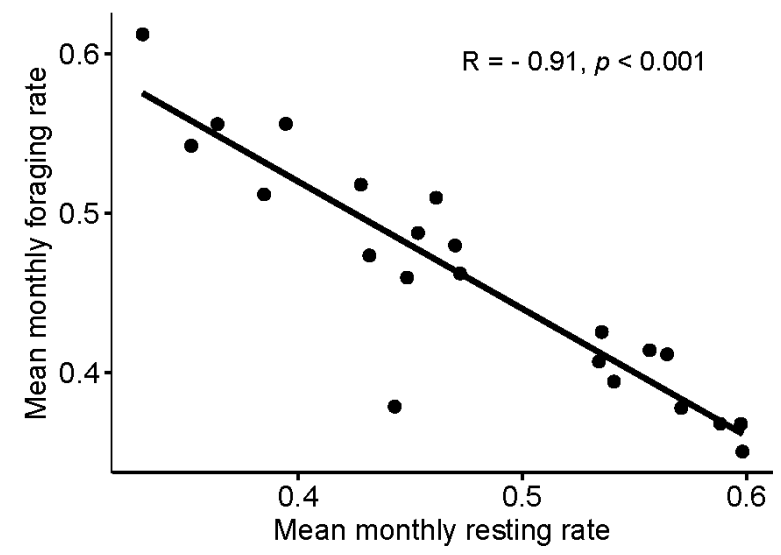

d)

$\Delta$ Temp vs HR

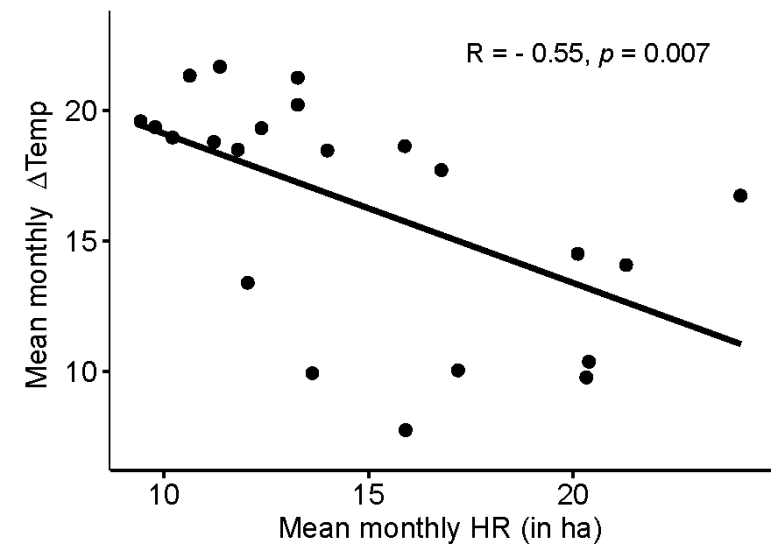

Figure S4 Pearson correlations among temperature differences, ranging and activity patterns. Data points represent monthly means of the whole study population. Solid lines represent regression lines of Pearson correlations. 
(Quinn and Keough, 2002). We included food availability as control variable. Temperature differences

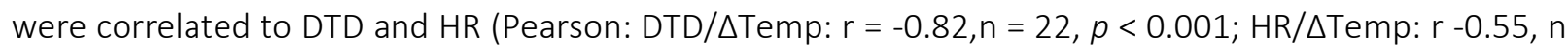
$=22, p<0.001$, Figure S4c \& d), which is why we excluded it from the model. We conducted full-reduced model comparisons, with reduced models containing intercepts, control variables (i.e. food availability), random effects and random slopes. We included group and animal ID as random effects; and DTD or HR, foraging or resting rates, and food availability as random slopes within group and ID, respectively.

Table S22 Correlations of fGCMs (In-transformed) with mean monthly daily travel distances and foraging rates of seven groups of Verreaux's sifakas; results of the full model (GLMM; $\left.N_{\text {observations }}=630, N_{I D}=41\right)$.

\begin{tabular}{|c|c|c|c|c|c|c|c|c|c|}
\hline Term & Est & SE & Lower $\mathrm{Cl}$ & Upper Cl & $\operatorname{Min}^{a}$ & $\operatorname{Max}^{a}$ & $\chi^{2 b}$ & $d f$ & $P$ \\
\hline (Intercept) & -1.235 & 0.042 & -1.325 & -1.141 & -1.260 & -1.206 & $\mathrm{c}$ & c & $\mathrm{c}$ \\
\hline DTD $^{d}$ & -0.234 & 0.038 & -0.325 & -0.15 & -0.263 & -0.206 & 12.694 & 1 & $<0.001$ \\
\hline Foraging rate & -0.047 & 0.016 & -0.102 & 0.009 & -0.057 & -0.037 & 6.563 & 1 & 0.010 \\
\hline Food availability ${ }^{f}$ & -0.061 & 0.029 & -0.132 & 0.012 & -0.078 & -0.033 & 3.467 & 1 & 0.063 \\
\hline 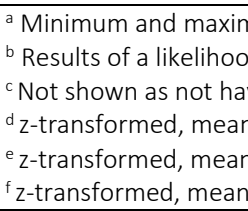 & $\begin{array}{l}\text { model est } \\
\text { test comp } \\
\text { neaningfu } \\
\text { of the or } \\
\text { of the or } \\
\text { of the or }\end{array}$ & $\begin{array}{l}\text { tes obtai } \\
\text { g the full } \\
\text { erpretati } \\
\text { al values } \\
\text { al values } \\
\text { al values }\end{array}$ & $\begin{array}{l}\text { d when drop } \\
\text { odel with a r } \\
\text { See footnot } \\
\text { ere } 918 \text { and } 2 \\
\text { ere } 0.454 \text { anc } \\
\text { ere } 2.800 \text { and }\end{array}$ & $\begin{array}{l}\text { ig levels of ra } \\
\text { uced model I } \\
\text { of Table S1 f } \\
3 \text {, respectivel } \\
.192 \text {, respect } \\
962, \text { respect }\end{array}$ & $\begin{array}{l}\text { om effects } \\
\text { king the re } \\
\text { details. } \\
\text { ly }\end{array}$ & $\begin{array}{l}\text { e at a tim } \\
\text { ctive term }\end{array}$ & & & \\
\hline
\end{tabular}

Table S23 Correlations of fGCMs (In-transformed) with mean monthly home range sizes and resting rates of seven groups of Verreaux's sifakas; results of the full model (GLMM; $\left.N_{\text {observations }}=630, N_{I D}=41\right)$.

\begin{tabular}{|c|c|c|c|c|c|c|c|c|c|}
\hline Term & Est & SE & Lower $\mathrm{Cl}$ & Upper Cl & $\operatorname{Min}^{a}$ & $\operatorname{Max}^{a}$ & $\chi^{2 b}$ & $d f$ & $P$ \\
\hline (Intercept) & -1.229 & 0.066 & -1.375 & -1.09 & -1.279 & -1.189 & c & c & c \\
\hline$H R^{d}$ & -0.264 & 0.048 & -0.389 & -0.142 & -0.310 & -0.239 & 11.626 & 1 & 0.001 \\
\hline Resting rate $\mathrm{e}^{\mathrm{e}}$ & 0.077 & 0.021 & 0.012 & 0.143 & 0.060 & 0.093 & 7.562 & 1 & 0.006 \\
\hline Food availability ${ }^{f}$ & -0.101 & 0.031 & -0.179 & -0.030 & -0.114 & -0.068 & 6.548 & 1 & 0.011 \\
\hline \multicolumn{10}{|c|}{$\begin{array}{l}{ }^{a} \text { Minimum and maximum of model estimates obtained when dropping levels of random effects one at a time } \\
\text { b Results of a likelihood ratio test comparing the full model with a reduced model lacking the respective term } \\
{ }^{c} \text { Not shown as not having a meaningful interpretation. See footnotes of Table S1 for details. } \\
\text { d z-transformed, mean and SD of the original values were } 14.163 \text { and } 6.310 \text {, respectively } \\
\text { e z-transformed, mean and SD of the original values were } 0.482 \text { and } 0.195 \text {, respectively } \\
{ }^{f} \text { z-transformed, mean and SD of the original values were } 2.800 \text { and } 0.962 \text {, respectively }\end{array}$} \\
\hline
\end{tabular}


a)

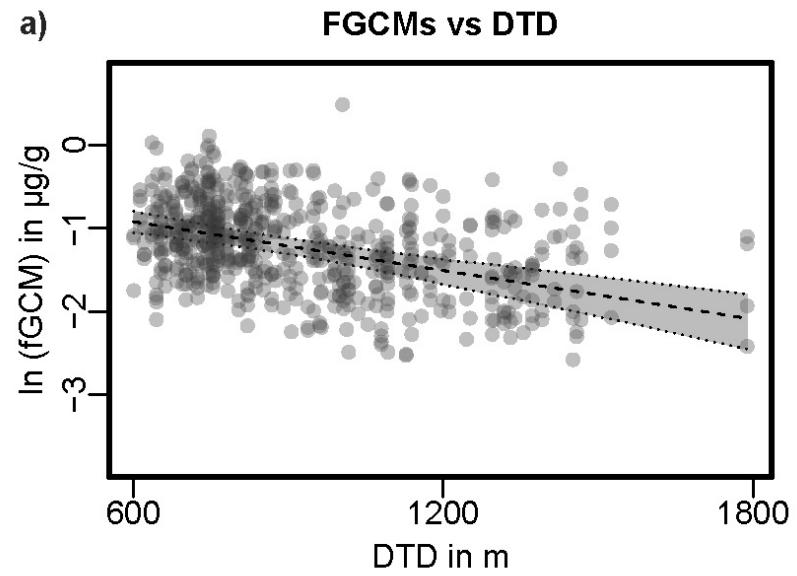

c)

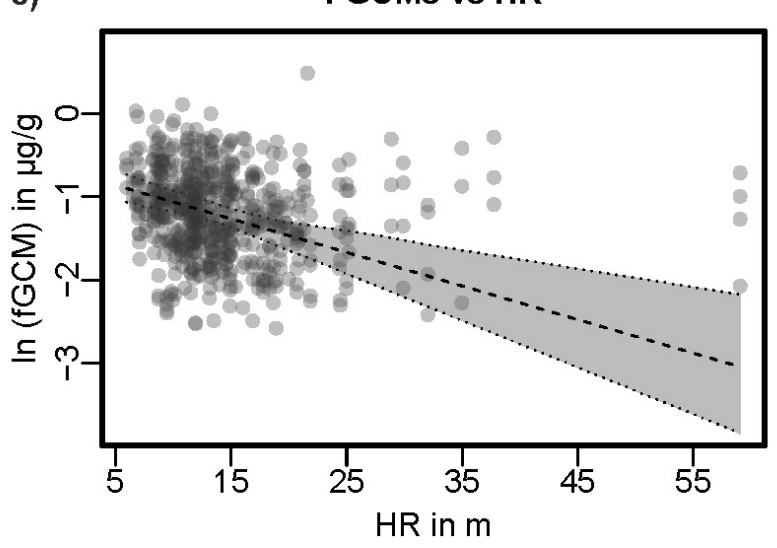

b) FGCMs vs foraging rate

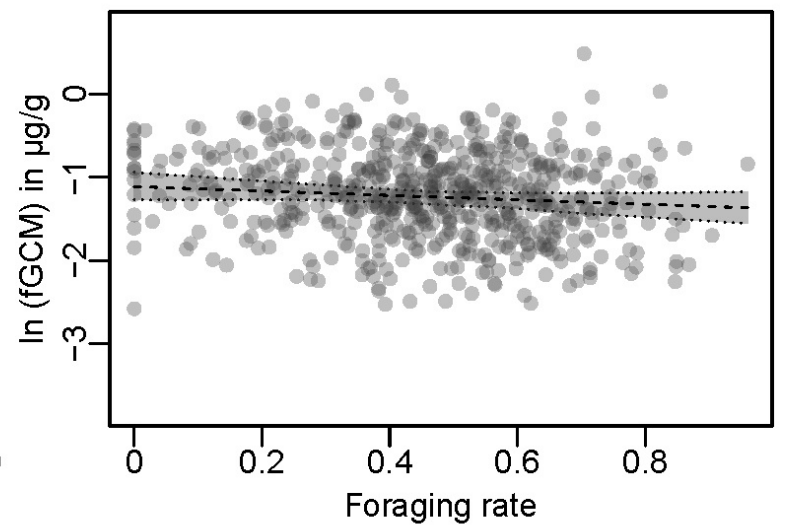

d) FGCMs vs resting rate

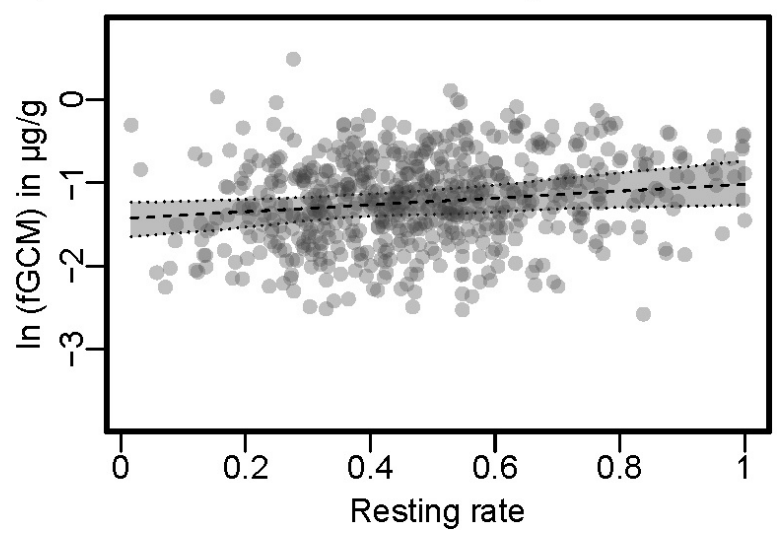

Figure S5 Correlations of individual monthly fGCMs with ranging and activity patterns. Data points represent monthly means of the corresponding variable. Dashed lines represent regression lines of the models and grey areas mark confidence intervals. Highlighted graphs indicate significant correlations. 


\section{Chapter 3}

Study II

\section{Dynamics and Determinants of Glucocorticoid Metabolite Concentrations in Wild Verreaux's Sifakas}

\footnotetext{
with Claudia Fichtel ${ }^{1,3}$, Michael Heistermann ${ }^{4}$, and Peter M. Kappeler ${ }^{1,2,3}$

${ }^{1}$ Behavioral Ecology \& Sociobiology Unit, German Primate Center, Leibniz Institute for Primate Research, Göttingen, Germany

2 Dept. Sociobiology /Anthropology, Johann-Friedrich-Blumenbach Institute of Zoology and Anthropology, University Göttingen, Göttingen, Germany

${ }^{3}$ Leibniz Science Campus “Primate Cognition", Göttingen, Germany

${ }^{4}$ Endocrinology Laboratory, German Primate Center, Leibniz Institute for Primate Research, Göttingen, Germany
}

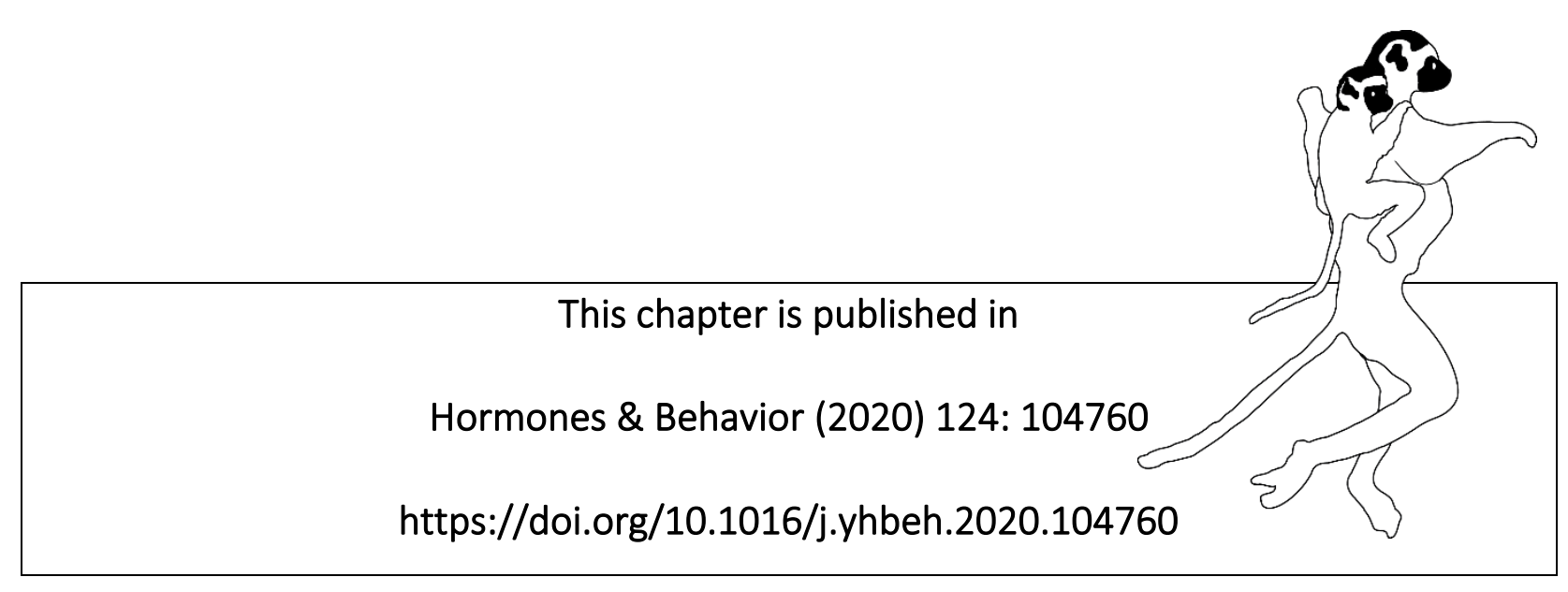




\title{
Graphical Abstract
}

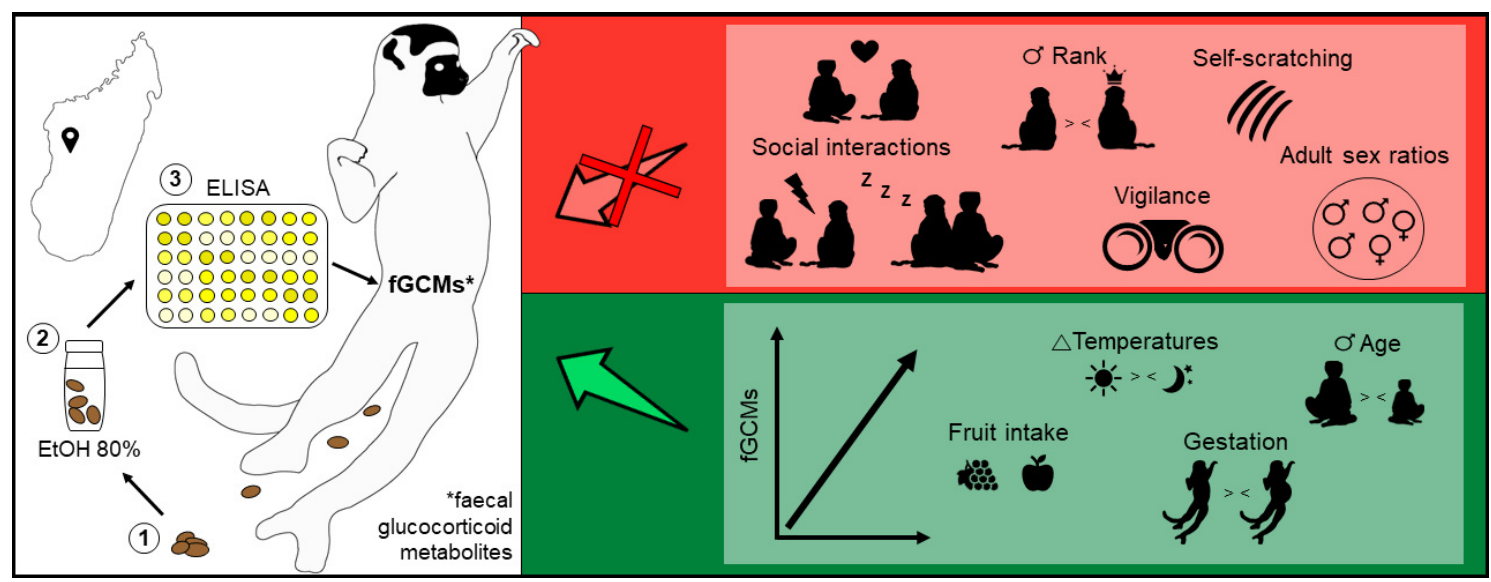

\begin{abstract}
Glucocorticoids have wide-ranging effects on animals' behaviour, but many of these effects remain poorly understood because numerous confounding factors have often been neglected in previous studies. Here, we present data from a 2-year study of 7 groups of wild Verreaux's sifakas (Propithecus verreauxi), in which we examined concentrations of faecal glucocorticoid metabolites (fGCMs, $n=2350$ samples) simultaneously in relation to ambient temperatures, food intake, rank, reproduction, adult sex ratios, social interactions, vigilance and self-scratching. Multi-variate analyses revealed that fGCM concentrations were positively correlated with increases in daily temperature fluctuations and tended to decrease with increasing fruit intake. fGCM concentrations increased when males were sexually mature and began to disperse, and dominant males had higher fGCM concentrations than subordinate males. In contrast to males, older females showed a non-significant trend to have lower fGCM levels, potentially reflecting differences in male and female life-history strategies. Reproducing females had the highest fGCM concentrations during late gestation and had higher fGCM levels than non-reproducing females, except during early lactation. Variation in $\mathrm{fGCM}$ concentrations was not associated with variation in social interactions, adult sex ratios, vigilance and self-scratching. Altogether, we show that measures of glucocorticoid output constitute appropriate tools for studying energetic burdens of ecological and reproductive challenges. However, they seem to be insufficient indicators for immediate endocrinological responses to social and nonsocial behaviours that are not directly linked to energy metabolism.
\end{abstract}




\title{
Chapter 4
}

Study III

\section{Exploring causes for gut microbiome variation among groups of wild Verreaux's sifakas}

\author{
with Dominik Schneider ${ }^{1}$, Claudia Fichtel2,4, Rolf Daniel ${ }^{1}$, \\ and Peter M. Kappeler $2,3,4$
}

\footnotetext{
${ }^{1}$ Genomic and Applied Microbiology and Göttingen Genomics Laboratory, Institute of Microbiology and Genetics, University Göttingen, Göttingen, Germany

${ }^{2}$ Behavioral Ecology \& Sociobiology Unit, German Primate Center, Leibniz Institute for Primate Research, Göttingen, Germany

${ }^{3}$ Dept. Sociobiology /Anthropology, Johann-Friedrich-Blumenbach Institute of Zoology and Anthropology, University Göttingen, Göttingen, Germany

${ }^{4}$ Leibniz Science Campus “Primate Cognition”, Göttingen, Germany
}

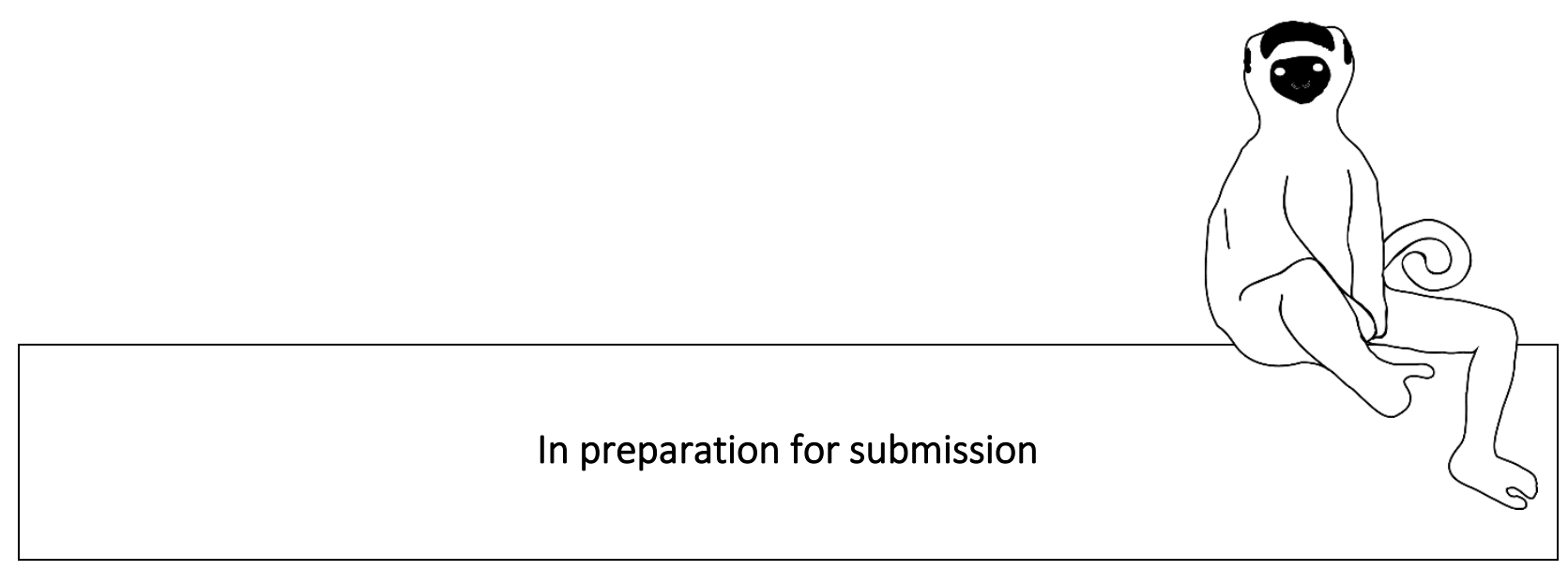




\section{Abstract}

The gut microbiota plays a fundamental role for animal health and the identification of ecological and evolutionary processes shaping host-associated microbial communities constitutes a key topic in molecular ecology. An increasing number of studies have reported associations between group living and gut microbial convergence, however, the relative contributions of environmental, intrinsic and social factors to these links remain debated. Here, we examine the drivers of gut microbial convergence among seven neighbouring groups of wild Verreaux's sifakas (Propithecus verreauxi) - a group-living primate from Madagascar. Over four field seasons, we collected 519 faecal samples of 41 animals and determined gut microbial composition and diversity via 16S rRNA gene sequencing. We correlated microbial data with observations of social interactions, maternal relatedness, diet, habitat structure and overlap, while controlling for seasonality. Groups differed in both, composition and diversity of their gut microbiomes. In contrast to most primate studies, we found kinship to play an important role for within-group microbial homogeneity, while we did not find an effect of dyadic social contact. None of the environmental predictors explained variation in microbial communities between groups. Altogether, we find that environmental factors define the general set-up of species- or population-specific gut microbiota, while kinship has important implications for individual microbial patterns. The degree to which social interactions shape individual gut microbiota seems to differ with species' social systems, which determine opportunities for microbial dispersals. More comprehensive research on taxa with varying social systems will help to understand the co-evolutionary dynamics of host sociality and microbial transmission strategies. 


\section{Introduction}

As animal life emerged and evolved in a microbial world, it is not surprising that their bodies are home to diverse assemblages of microbes. These microbes have important functions in the context of maintaining and shaping their host's condition and health. Gut bacteria, for example, are involved in digestion and energy harvest (Gill et al., 2006; Turnbaugh et al., 2006b), they interact with the immune system (Chow et al., 2010; Kinross et al., 2011), and cases of dysbiosis are associated with lower serum immunoglobulin levels, decreased lymphocytes (Round and Mazmanian, 2009) and several diseases, like diabetes (Boerner and Sarvetnick, 2011) and Crohn's disease (Knights et al., 2013). However, the relationship between gut microbiota and their hosts is bi-directional and dynamic, as the microbial compositions are shaped by various social, genetic and environmental factors (Clayton et al., 2018; Gogarten et al., 2018; Grieneisen et al., 2019; Heitlinger et al., 2017; Ren et al., 2017). In the last decade, much progress has been made towards identifying the forces that shape host-microbiota relationships in humans and laboratory animals, but only more recently attention was given to studies of wild animals. Yet, identifying the factors that drive differences in gut microbiota composition in wild populations is critical for understanding how microbes impact animals' health, ecology and evolution under natural conditions (Björk et al., 2019), not the least because gut microbiomes play a key role in mediating links between sociality and health (Archie and Theis, 2011; Clayton et al., 2018; Kappeler et al., 2015).

\section{Social drivers of gut microbiome communities}

An increasing number of studies across different taxa, ranging from insects over birds to mammals, has reported associations between microbiome composition and social co-residency (Ezenwa et al., 2012; Levin et al., 2016; Martinson et al., 2011; Raulo et al., 2017; Song et al., 2013; Springer et al., 2017; Trosvik et al., 2018). Increased physical contact between group members facilitates the transmission of microorganisms and is a prevalent mechanism for shaping distinct group microbiomes (Kulkarni and Heeb, 2007; Moeller et al., 2016; Raulo et al., 2017; Tung et al., 2015). It may also facilitate transmission of pathogen resistance-enhancing bacteria (Ezenwa et al., 2016; Hughes et al., 2002; Koch and SchmidHempel, 2011; Kuthyar et al., 2019) and can increase microbial diversity, which is suggested to correlate with resilient immunity (Hooper et al., 2012; Lozupone et al., 2012). Within groups, affiliative interactions between individuals can result in convergence in microbial communities, as shown in baboons (Papio cynocephalus), howler monkeys (Alouatta pigra) and humans (Amato et al., 2017a; Lax et al., 2014; Tung et al., 2015). However, group membership or social contact are not always linked to variation in gut microbiome compositions, as reported in fruit bats (Rousettus aegyptiacus) (Kolodny et al., 2019) or sooty mangabeys (Cercocebus atys) (Gogarten et al., 2018).

Group size represents another aspect of sociality that can alter microbial compositions and diversities (Turnbull et al., 2011) because variation in group size can affect frequencies of social 
interactions and physical contacts (Keiser et al., 2018; Nunn et al., 2015). Accordingly, population density in plateau pikas (Ochotona curzoniae) and group size in yellow baboons were found to be positively correlated with microbiome diversity (Grieneisen et al., 2017; Li et al., 2016), whereas group size was negatively correlated in African social spiders (Stegodyphus dumicola) (Keiser et al., 2018) or not correlated in red-bellied lemurs (Eulemur rubriventer) (Raulo et al., 2017). Hence, the effects of social contact and group size on microbial diversity remain ambiguous across taxa. Moreover, the relative contributions of sociality in shaping gut microbial communities against the background of variation in genetic and environmental factors remain little understood, especially in wild populations.

\section{Kinship as driver of gut microbiome communities}

Kinship can influence similarity of gut microbiomes among individuals (Lombardo, 2008; Yuan et al., 2015; Zoetendal et al., 2001). There are three known mechanisms underlying this effect: 1) heritability of microbiomes, i.e. inheritance of certain genetic loci, under the premise that host genomes dictate to some degree abundances of microbes (Goodrich et al., 2014; Kovacs et al., 2011; Opstal and Bordenstein, 2015); 2) direct maternal transmission to offspring in utero (e.g. Collado et al., 2016; but see Perez-Muñoz et al., 2017) or during delivery (Bokulich et al., 2016; Gregory et al., 2015); or 3) transmission via physical contacts in the contexts of parental care and social behaviours (Funkhouser and Bordenstein, 2013; Lombardo, 2008). Microbial patterns obtained via one of the afore-mentioned transmission modes can persist into adulthood (Nelson et al., 2013; Palmer et al., 2007; Ren et al., 2017). Yet, there is little knowledge on the contribution of kinship to microbial composition and diversity in wild populations. So far, studies in red squirrels (Tamiasciurus hudsonicus), cheetahs (Acinonyx jubatus) and gopher tortoises (Gopherus polyphemus) (Yuan et al., 2015; Ren et al., 2017; Wasimuddin et al., 2017) found strong indications for kinship effects on gut microbiomes, whereas studies in baboons, chimpanzees and howler monkeys found either no or only weak links (Amato et al., 2017a; Degnan et al., 2012b; Grieneisen et al., 2017). This variation may be due to the fact that groups contain variable numbers of related individuals, and the frequency and nature of social interactions varies among species.

\section{Environmental drivers of gut microbiome communities}

Environmental factors, like host diet and habitat heterogeneity, are well known to affect animals' gut microbiomes as well (Greene et al., 2019b; Kohl et al., 2017; Perofsky et al., 2019; Rothschild et al., 2018; Smith et al., 2015). For example, short-term changes in diet can rapidly alter bacterial abundances in humans, primates and carnivores (Amato et al., 2015; David et al., 2014; Williams et al., 2013). Moreover, primates living in degraded habitats have less diverse microbiomes (Amato et al., 2013; Barelli et al., 2015), and habitat heterogeneity alters microbiome compositions in elephants and baboons (Chiyo et al., 2014; Grieneisen et al., 2017). Because group members share the same habitat and similar diets, distinct gut microbiota might reflect differences in food availability or habitat type between groups' home ranges 
(Amato et al., 2013; Grieneisen et al., 2019). In species with overlapping home ranges, neighbouring groups might therefore share more similar microbiomes than groups living in different or distant areas. However, the relative importance of environmental or social factors is still debated, with some studies suggesting environmental factors as main contributors to microbiome convergence between co-residing conspecifics (Degnan et al., 2012b; Grieneisen et al., 2019), whereas others propose social interactions to have a stronger impact (Goodfellow et al., 2019; Wikberg et al., 2019).

\section{Study design}

Here, we simultaneously examined several potential drivers of between-group variation in gut microbial compositions in a wild population of Verreaux's sifakas (Propithecus verreauxi). Two previous studies of this species indicated that group membership is reflected in individuals' gut microbiome compositions (Perofsky et al., 2017; Springer et al., 2017). Springer et al. (2017) investigated, next to the impact of group membership, effects of seasonal variation in diet and the effects of intrinsic factors on microbial composition and diversity in the same study population. They found a clear pattern of seasonal variation, especially on the Firmicutes-Bacteroidetes ratio, while there were no or only weak influences of sex, age class and female reproductive stage. Perofsky et al. (2017) provided a snapshot perspective on the gut microbiomes of a different sifaka population and examined impacts of grooming networks, diet, habitat overlap and host intrinsic factors on microbial similarity and within-host diversity. They found no effect of diet, habitat overlap, kinship or within-group social interactions on microbial similarity but found positive links between age and grooming interactions with microbiome diversity.

To better understand the relative importance of environmental, intrinsic and social factors that drive gut microbial convergence among group members, we set out this study to examine the effects of social interactions, maternal relatedness, diet, habitat structure, habitat overlap and seasonality. We repeatedly collected and analysed faecal samples of up to 41 individuals from 7 different groups during the early and late dry season in two subsequent years. Additionally, we describe Verreaux's sifakas' core gut microbiota. The core microbiota includes only those taxa that are present in the majority of analysed hosts (Hamady \& Knight 2009) and is thought to be involved in the gut microbiomes' general functions (Shade \& Handelsman 2012). Thus, we contribute an independent observation to the few comprehensive and longitudinal studies of the determinants of the gut microbiome in wild animals.

We expected to find distinct gut microbial communities per group for each field season (Perofsky et al., 2017; Springer et al., 2017). Moreover, within groups, animals that spent more time in body contact, including grooming, were expected to share more similar microbial communities with each other than with other group members (Tung et al., 2015). Maternally related animals should share more similar gut microbiomes with each other than with unrelated individuals, even though the effects might be weak (Amato et al., 2017a; Degnan et al., 2012b; Grieneisen et al., 2017). In addition, we predicted neighbours 
and groups inhabiting more similar habitats and those sharing more similar diets, to exhibit greater microbial similarity. Besides, we expected larger groups to exhibit a greater microbiome diversity, but only if members of larger groups engage in more intimate social interactions. Finally, as indicated by a previous study in the same population (Springer et al., 2017), seasonal variation in diet, especially in terms of relative fruit and leave intake, should alter gut bacterial compositions and diversity should be greater during the late dry season as response to an increased fibre intake.

\section{Material \& Methods}

\section{Study site}

This study was carried out in Kirindy Forest, Western Madagascar $\left(44^{\circ} 39^{\prime} \mathrm{E}, 20^{\circ} 03^{\prime} \mathrm{S}\right)$. The study area is managed by the Centre National de Formation, d'Etudes et de Recherche en Environnement et Foresterie (CNFEREF) and belongs to a research station operated by the German Primate Center. Kirindy Forest is a dry deciduous forest and subject to pronounced seasonality, with a short hot, wet season (November to March) and a long dry season (April to October) (Kappeler and Fichtel, 2012).

\section{Study species}

Verreaux's sifakas are diurnal, frugi-folivorous and arboreal lemurs endemic to Madagascar. They live in multi-male multi-female groups with group sizes ranging between 2 and 12 individuals in our study population (Kappeler and Fichtel, 2012). Their home ranges are stable over years and partially overlap with those of neighbouring groups, yet, they include core areas of exclusive use (Benadi et al., 2008; Koch et al., 2016a). Additionally, in contrast to anthropoid primates, sifakas groom each other orally rather than manually, which may enhance the possibility for bacterial transmission between individuals. All animals are habituated to observers and individually marked. One of the groups, group $\mathrm{M}$, only entered the study area by the end of 2016, so that data for this group were only available for study year 2017.

\section{Home range features}

Forest inventories of 10 randomly selected square plots ( $25 \times 25 \mathrm{~m}$; only 6 plots for group F 1 ) within the home ranges of each group were conducted in 2012 (Koch et al., 2017) and 2016 (Rudolph et al., 2019). We identified all trees with diameters at breast height larger than $5 \mathrm{~cm}$, resulting in a data set comprising 12,177 trees of 168 different species found in 66 phenology plots (for details see Rudolph et al. 2019).

\section{Behavioural observations}

Between April 2016 and March 2018, we conducted focal animal sampling on all adults and juveniles (age $>9$ months). Observations lasted $1 \mathrm{~h}$ per individual and were conducted for $3 \mathrm{~h}$ in the morning and $3 \mathrm{~h}$ in the afternoon in an alternating order. We continuously recorded social behaviours (i.e. allogrooming, play, body contact, proximity of $<1 \mathrm{~m}$, aggression), including the identity of involved conspecific(s), and non-social behaviours, like feeding, locomoting, resting, self-scratching and auto-grooming. We 
additionally recorded the identity of feeding plants and parts. As our study involved focal animal observations, it was not possible to record data blind.

\section{Home range overlap}

In a previous study (Rudolph et al., 2019), we assed home range sizes of each group with GPS collars (eobs, Grünwald, Germany) by equipping one male per group during annual captures (for details on capture procedures see Kappeler and Fichtel 2012). On average, we recorded GPS data for 651 days with a mean of 21400 GPS locations per group. To estimate home range overlap among groups for each of the four field seasons, we used the function kerneloverlaphr of the adehabitatHR package (Calenge, 2006) in R ( $R$ Version 3.6.1, R Core Team, 2018). Figure S1 illustrates home range overlaps among groups.

\section{Bacterial gut microbiome analyses}

We collected and analysed 519 faecal samples during four field seasons $(12.7 \pm 3.6$ (mean \pm SD) total samples per animal; $\varnothing 3.9$ samples per animal per season), covering the early dry season (April-May 2016/17) and the late dry season (September-October 2016/17) twice. Samples were only collected when they could be distinctively assigned to an individual. We stored samples in $2 \mathrm{ml}$ polypropylene tubes containing $1 \mathrm{ml}$ RNAlater (Thermo Fisher Scientific, Waltham, MA, USA) at ambient temperature for 24h. Afterwards, samples were stored at $-20^{\circ} \mathrm{C}$ and shipped to Germany, where further analyses ensued.

\section{Extraction of DNA, amplification, and sequencing of 165 rRNA genes}

We extracted DNA from approximately $100 \mathrm{mg}$ of faecal samples using the PowerSoil DNA isolation kit (MoBio, Carlsbad, USA) following the instructions of the manufacturer. However, to ensure complete homogenization of the faecal samples, cells were mechanically disrupted with a FastPrep-24 Classic Grinder (MP Biomedicals, Santa Ana, California, USA). Samples were homogenized for $20 \mathrm{~s}$ at $6.5 \mathrm{~m} / \mathrm{s}$. Bacterial 16S rRNA gene amplicons were generated using the forward and reverse primers S-D-Bact-0341b-S-17 (5'- CCTACGGGNGGCWGCAG-3') and S-D-Bact-0785-a-A-21 (5'-GACTACHVGGGTATCTAATCC-3') (Klindworth et al., 2013), including the Illumina MiSeq sequencing adaptors and target the V3 to V4 variable regions of the $16 \mathrm{~S}$ rRNA gene. PCR reaction mixtures (total volume $50 \mu \mathrm{l}$ ) contained $1 \mathrm{U}$ Phusion high fidelity DNA polymerase (Biozym Scientific, Oldendorf, Germany), $2.5 \mu \mathrm{I}$ DMSO (5\%), $1 \mu \mathrm{l}$ of forward and reverse 16S rRNA gene primers $(10 \mu \mathrm{M}), 1 \mu \mathrm{dNTP}(10 \mathrm{mM}), 0.2 \mu \mathrm{l} \mathrm{MgCl} 2(50 \mathrm{mM})$, and $25 \mathrm{ng}$ of isolated DNA. Thermal cycling conditions were as follows: initial denaturation for $1 \mathrm{~min}$ at $98^{\circ} \mathrm{C}, 25$ cycles at $98{ }^{\circ} \mathrm{C}$ for $45 \mathrm{~s}, 55^{\circ} \mathrm{C}$ for $45 \mathrm{~s}$, and $72{ }^{\circ} \mathrm{C}$ for $30 \mathrm{~s}$, and a final extension at $72{ }^{\circ} \mathrm{C}$ for $5 \mathrm{~min}$. We included negative controls (without template) and positive controls (with genomic E. coli DH5 $\alpha$ DNA) in all PCRs. We used agarose gel electrophoresis to verify correct amplicon size ( $\sim 50 \mathrm{bp})$. PCR reactions were performed in triplicates for each sample, then pooled in equimolar amounts and purified using MagSiNGS PREP Plus (Steinbrenner, Wiesenbach, Germany) as described by the supplier. Nextera DNA Library 
Prep kits were used for indexing PCR products according to the manufacturer's manual (Illumina), followed by dual-indexed paired-end sequencing with the Illumina MiSeq platform ( $2 \times 300 \mathrm{bp})$ and v3 chemistry (Illumina, San Diego, CA, USA).

\section{Bioinformatic processing of 165 rRNA gene amplicon sequences}

Paired-end sequencing data from the Illumina MiSeq were quality-filtered with fastp (v0.19.4) (S. Chen et al., 2018) using default settings with the addition of an increased per base phred score of 20, base pair corrections by overlap (-c), as well as $5^{\prime}$ - and $3^{\prime}$-end read trimming with a sliding window of 4 , a mean quality of 20 and minimum sequence size of $50 \mathrm{bp}$. After quality control, the paired-end reads were merged using PEAR (v0.9.11) (Zhang et al., 2014) and primers clipped using cutadapt (v1.18) (Martin, 2011) with default settings. Sequences were then processed using VSEARCH (V2.9.1) (Edgar, 2010). Processing included sorting and size-filtering of the paired reads to $\geq 300 \mathrm{bp}$ (--sortbylength -minseqlength 300) and dereplication (--derep_fulllength). Dereplicated amplicon sequence variants (ASVs) were denoised with UNOISE3 using default settings (--cluster_unoise -minsize 8) and chimeras were removed (--uchime3_denovo). An additional reference-based chimera removal was performed (-uchime_ref) against the SILVA SSU NR database (v132) (Quast et al., 2013). Raw reads were mapped to ASVs (--usearch_global-id 0.97). Finally, we taxonomically classified ASVs with BLAST 2.7.1+ (Altschul et al., 1990) against the SILVA SSU v132 database and removed chloroplasts and extrinsic domains from the data set.

The following analyses were performed on data normalised with the GMPR method (v0.1.3) (L. Chen et al., 2018) and conducted in R (R v3.6.1, R Core Team, 2018). To assess alpha diversity, i.e. the bacterial diversity within samples, we calculated Faith's Phylogenetic Diversities (PD) (Faith, 1992) with the picante package (v1.8) to assess microbial phylogenetic representation, and Shannon's indices (Shannon, 1948) to examine community evenness, with the diversity function of the vegan package (v2.56) (Oksanen et al. 2016). For examinations of beta diversity, i.e. variance in community composition among samples, we computed two measures: Bray-Curtis dissimilarities and generalized UniFrac distances (GUniFrac). Bray-Curtis dissimilarities detect differences in microbial abundances, which we calculated with the vegdist function of the vegan package. GUniFrac distances also detect microbial abundances but further include information on counts and adjusted weights of phylogenetic branch lengths (Chen et al., 2012). We computed GUniFrac distances with the GUniFrac function of the GUniFrac package (version 1.1) (Chen et al., 2012). Core gut microbiota were determined following definitions of prior studies, i.e. ASVs present in $\geq 90 \%$ of samples were categorized as core ASVs (Ainsworth et al., 2015; Grieneisen et al., 2017; Li et al., 2013).

Group differences in ASV-based community composition were visualised with nonmetric multidimensional scaling (NMDS) ordinations based on GUniFrac and Bray-Curtis distance matrices. 
Additionally, we built heat trees to illustrate and examine variations in microbiome phylotypes between groups with the metacoder package (Foster et al., 2017). Heatmaps were built with the ampvis2 package (version 2.5.5) (Skytte et al., 2018).

\section{Statistical analyses}

All statistical analyses were conducted in $\mathrm{R}$.

\section{Mantel tests - Beta diversity and group membership}

We examined the relationship between group membership and beta diversity with Mantel tests using 1,000 permutations, including the original data as one permutation. We conducted four Mantel tests, each including only samples of one of the four field trips. Animal ID was assigned to the samples to control for repeated measures. The test statistic yielded the mean absolute differences in dissimilarities within and between groups. We determined p-values as the proportion of permutations that resulted in larger test statistics than or equal to the test statistics of the original data. The unpublished functions for this analysis were kindly provided by Roger Mundry.

\section{$\underline{\text { GLMM - Beta diversity within groups }}$}

To investigate whether samples from the same individual are more similar than samples between group members, we computed a generalized linear mixed model (GLMM) (Baayen et al., 2008) from the Ime4 package (version: 1.1.21) (Bates et al., 2012) with the optimizer "bobyqa". Mean GUniFrac distances per ID dyad and field season were used as response, the factor "same individual" (yes or no) was used as predictor, individual dyads and group ID were used as random effects and field season as random slope. To control for seasonal variation, we included field season as fixed effect. We included random slopes to keep type I error rates at the nominal level of 5\% (Barr et al., 2013). P-values for individual effects were based on likelihood ratio tests comparing the full with the respective null models using the drop 1 function (Barr et al., 2013). In this and all following GLMMs, we controlled for assumptions of normal distributions, homoscedasticity and collinearity, and we checked for model stability. Comparisons between full and null or reduced models were done with likelihood ratio tests ( $R$ function ANOVA with argument test set to "Chisq") (Dobson, 2002; Forstmeier and Schielzeth, 2011). Null models contained only intercepts, random effects and random slopes; reduced models additionally contained assigned control factors. We obtained effect sizes of the full models for the entirety of fixed and random effects with the function r.squaredGLMM of the package MuMIn (version 1.43.6) (Barton, 2018). Confidence intervals were assessed with parametric bootstrapping using an adjusted bootMer function from the Ime4 package. Roger Mundry also kindly provided this adjusted function. 


\section{$\underline{\text { GLMM - Beta diversity and body contact within groups }}$}

We examined whether group members that spent more time in body contact, including grooming and activities (i.e. feeding, resting) in body contact, share more similar microbiome communities. Behavioural data for this analysis were collected from January to May 2017 and June to October 2016/17, i.e. covering the time of three out of four field seasons plus the 3 months prior to the respective field seasons (i.e. data for the field season April-May 2016 were not included in this analysis as there were no behavioural observations conducted from January to March 2016). Observations of juveniles usually start in the first April after their birth, when they are about 9 months old. Hence, to ensure a balanced data set, juveniles were excluded from the early dry season 2017, as there were no observations between January and March for these animals. During this time, we collected 1,364 h of behavioural data of which Verreaux's sifakas spent on average $3.6 \pm 1.6 \mathrm{~min} / \mathrm{h}$ (mean $\pm \mathrm{SD}$ ) in body contact. We computed a GLMM with the mean GUniFrac dissimilarities of individual dyads per field season as response and mean time spent in body contact (in $\mathrm{min} / \mathrm{h}$ ) as predictor. Individual dyads and group ID were used as random effects and field seasons as random slopes. To control for effects of field season and maternal relatedness (see below), we included both factors as fixed effects.

\section{$\underline{\text { GLMM - Beta diversity and maternal relatedness }}$}

We investigated the potential effect of genetic maternal relatedness on gut microbiome similarity among individuals. Maternal relatedness of older individuals was determined via genetic analyses in a prior study (1995 - 2005, Kappeler \& Schäffler 2007). For younger individuals, we used behavioural observations of mother-offspring dyads to determine relatedness (Kappeler and Fichtel, 2012). For 9 out of 41 individuals, mothers could not be assigned as they were absent during genetic data collection or adult individuals immigrated into the study population after 2005. These individuals were excluded from the analysis. We considered animals as maternally related if they were known to have one of the following degrees of kinship: mother-offspring, siblings or half-siblings, grandmother-grandchild, aunt/uncle-nephew/niece.

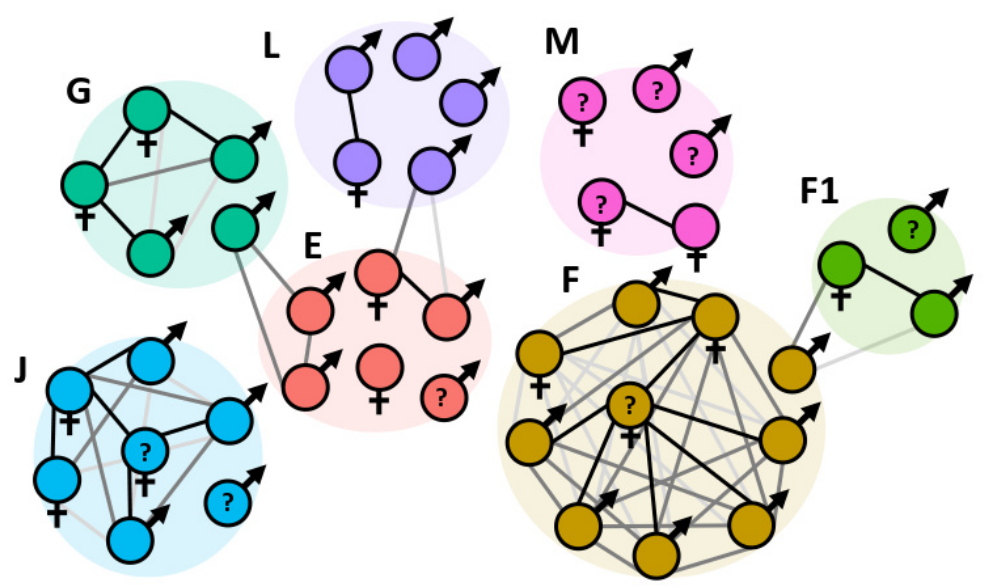

Figure 1 Group compositions and maternal relatedness within the study population. Circles represent individuals and indicate sex. Circles containing question marks indicate missing information on the respective animals' mothers. Lines indicate degrees of maternal relatedness. Black lines indicate mother-offspring dyads, dark grey lines indicate siblings and light grey lines indicate grandmother-grandchild or aunt/uncle - nephew/niece dyads. 
Figure 1 illustrates all known degrees of maternal relatedness within the study population. For statistical analysis, we computed a GLMM in the same manner as described above. Mean GUniFrac distances of individual dyads per field season were used as response, relatedness (yes or no) and the interaction between relatedness and group membership (yes or no) were used as predictors, individual dyad as random effect and field season as random slope. To control for effects of group ID and field season on GUniFrac distances, we included both factors as fixed effect.

Pearson's correlation - Beta diversity and home range dissimilarities among groups

We examined whether groups with ecologically more similar home ranges share more similar gut microbiomes. To estimate differences in home ranges, we computed Bray-Curtis dissimilarities among groups based on tree species abundances within each groups' home range. Next, we averaged GUniFrac distances for each group dyad from all samples. We then calculated a Pearson's correlation, examining the link between dyadic GUniFrac distances and dyadic habitat dissimilarity between groups ( $\mathrm{n}_{\text {Group dyads }}=$ 21).

\section{$\underline{\text { GLMM - Beta diversity and home range overlaps between groups }}$}

We investigated whether neighbouring groups with overlapping home ranges share more similar microbiomes than groups with non-overlapping home ranges with a GLMM. Mean GUniFrac distances per group dyad per field season were used as response, mean home range overlaps per field season were used as predictors, group dyad was used as random effect and field season as random slope. To control for seasonal variation in ranging patterns (see Rudolph et al., 2019), we included field season as fixed effect.

\section{$\underline{\text { GLMM - Beta diversity and diet dissimilarity between groups }}$}

Next, we examined whether groups with more similar diets share more similar gut microbiomes. To estimate differences in diets, we computed Bray-Curtis dissimilarities between groups based on proportions of feeding times spent on different plant species per field season. Data collection on feeding behaviour for this analysis was conducted from March to May 2017 and August to October 2016/17, resulting in a total of $280 \mathrm{~h}$ of feeding data. For the first field season (March to May 2016) no data on consumed plant species were recorded, which is why this analysis was only performed for the three remaining field seasons. We averaged GUniFrac distances and diet dissimilarities per group dyad per field season. For statistical analysis, we computed a GLMM in the same manner as described above. GUniFrac distances were used as response, mean diet dissimilarity as predictor, group dyad was used as random effect and field season and diet dissimilarity as random slopes. To control for seasonal variation in GUniFrac distances, we included field season as fixed effect. Moreover, we compared fruit and leave 
intake rates between groups and seasons in additional GLMMs described in the supplementary materials (Appendix Chapter 4).

\section{$\underline{\text { GLMM - Alpha diversity }}$}

We applied three GLMMs to examine effects of study year (2016 and 2017), season (early dry - late dry), group ID, group size, mean fruit and leave intake per season per animal and mean body contact rates per season per animal microbiome diversity. Phylogenetic diversity (PD) per sample was the response in all models. In the first model, study year, season, group ID and mean proportions of consumed fruits and leaves per field season were predictors, sex was a control factor, animal and group ID were random effects and season (early dry - late dry), fruit and leave intake and study year were used as random slopes. In the second model, group size was included as predictor, study year and sex were control factors, group and animal ID were random effects and group size and study year were random slopes. In the third model, we examined effects of time spent in body contact with group members via affiliative interactions (i.e. grooming, resting and feeding in contact). We used the mean time spent in body contact per field season as predictor, study year and sex as control factors, group and animal ID as random effects and body contact and study year as random slopes.

All analyses were additionally conducted with Bray-Curtis dissimilarities for beta diversity and Shannon indices for alpha diversity (Appendix Chapter 4).

\section{Seasonal variation in relative abundances of phyla}

We examined seasonal effects on microbiome composition by assessing changes in individual mean monthly abundances of core phyla within each of the two study years. We conducted Kruskal-Wallis tests, as data violated assumptions for parametric analyses, and carried out post hoc comparisons with Dunn's tests (Bonferroni correction) using the package FSA (version 0.8.22).

\section{Social network statistics - Comparisons with Perofsky et al. (2017)}

To better compare the effects of social interactions on gut microbiomes in our study population with the findings in a different population, we replicated some of the analyses from Perofsky et al. (2017). More precisely, we also constructed social networks based on grooming interactions and did so for each of three field seasons based on the data set described above. However, in contrast to Perofsky et al. (2017), not all study animals were connected to their group members in our grooming networks (Figure S6). Compared to other primates, sifakas devote little time to grooming interactions (Richard, 1985) and while grooming bouts occur frequently and on a daily basis, they usually remain short (Lewis, 2010). In our study, animals spent on average $18 \mathrm{~s} \pm 10$ s per hour (mean \pm SD) grooming. Most likely, our data did not detect all existing connections and we had to exclude unconnected individuals from the data set to run network statistics. Due to the missing data, there is limited scope for interpreting our results. 
Nevertheless, we provide details on methods and results and a brief discussion of grooming network analyses in the appendix of this chapter.

\section{Results}

519 faecal samples of 41 different individuals contained 5,995 bacterial ASVs and high quality 21,596,631 amplicon sequences. 2,966 of all ASVs could be taxonomically assigned and belonged to 12 phyla: Firmicutes (1,442), Bacteroidetes (927), Proteobacteria (174), Actinobacteria (155), Cyanobacteria (134), Verrucomicrobia (57), Synergistetes (43), Fibrobacteres (17), Spirochaetes (9), Tenericutes (4), Epsilonbacteraeota (3) and Armatimonadetes (1). About 50\% of all reads belonged to the 5 most common families: Prevotellaceae (17\%), Lachnospiraceae (15\%), Ruminococcaceae (7\%), Rikenellaceae (6\%) and Acidaminococcaceae (5\%) (Figure 2a).

\section{The core gut microbiota of Verreaux's sifakas}

The ASVs defined as core microbiome occurred in $96.4 \% \pm 3.2 \%$ (mean \pm SD) of all samples and in $95.9 \%$ $\pm 3.7 \%$ of all individuals (controlling for repeated sampling). The core comprised 214 ASVs of which 47 could not be assigned to any taxonomy. The remaining 167 ASVs belonged to eight different phyla: Firmicutes (119), Actinobacteria (13), Proteobacteria (12), Bacterioidetes (8), Synergistetes (5), Cyanobacteria (4), Fibrobacteres (3) and Verrucomicrobia (3). In the core microbiome, 50\% of all reads belonged to the 5 most common families: Lachnospiraceae (21\%), Rikenellaceae (11\%), Acidaminococcaceae (7\%), Ruminococcaceae (6\%) and Puniceicoccaceae (6\%) (Figure 2b).

\section{Beta diversity (GUniFrac)}

\section{Effects of host and group membership}

Samples from the same individuals were more similar than samples between group members (GLMM: $\chi^{2}$ $=26.366, \mathrm{df}=1, \mathrm{p}<0.001, \mathrm{R}_{\mathrm{m} / \mathrm{c}}=0.14 / 0.88$ ) (Table S1, Figure S2). Group membership impacted the gut microbiome as samples of group members were more similar to each other than samples from individuals living in different groups (Figure 3 and S3). This was true for each field season (Table 1). All groups exhibit very similar microbial compositions up to the family level and differences between groups appear mainly on the genus level and beyond. In more detail, groups seem to mainly differ within genera of the more

Table 1 Results of Mantel tests comparing GUniFrac distances between seven groups of Verreaux's sifakas.

\begin{tabular}{|c|c|c|c|c|c|}
\hline Season & $\mathrm{n}_{\text {samples }}$ & $\mathrm{n}_{\text {individuals }}$ & $\overline{\mathrm{X}}_{\text {same group }}$ & $\overline{\mathbf{X}}_{\text {different group }}$ & $P$ \\
\hline early dry 2016 & 92 & 29 & 0.121 & 0.155 & $<0.001$ \\
\hline late dry 2016 & 116 & 29 & 0.119 & 0.154 & $<0.001$ \\
\hline early dry 2017 & 155 & 39 & 0.125 & 0.147 & $<0.001$ \\
\hline late dry 2017 & 156 & 36 & 0.110 & 0.148 & $<0.001$ \\
\hline
\end{tabular}


abundant phyla, i.e. Firmicutes, Bacterioidetes, Actinobacteria, Cyanobacteria, Verrucomicrobia and Fibrobacteres (Figure 4). Moreover, heatmaps of the most common genera indicate noticeable variation between groups in terms of abundance and presence of unclassified ASVs (Figure 5).
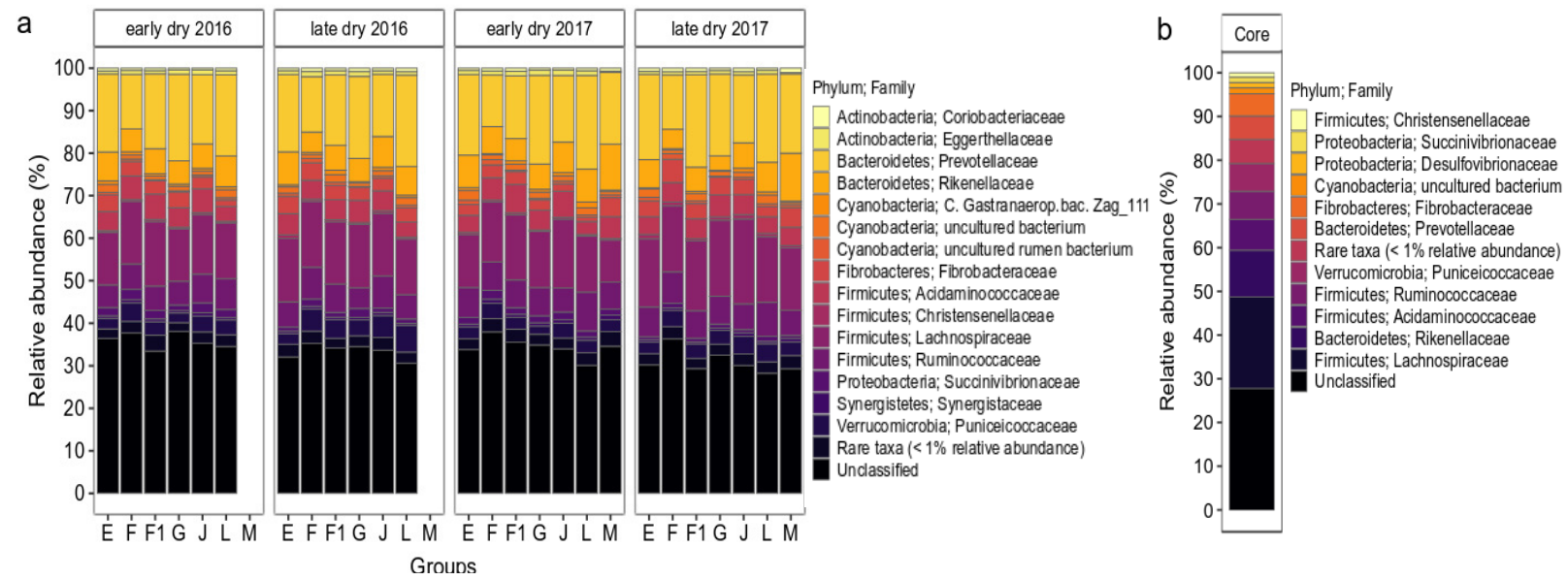

Figure 2 Relative abundances of a) all microbial families and b) core families. Data comprise 519 faecal samples of seven groups of wild Verreaux's sifakas during four field seasons.
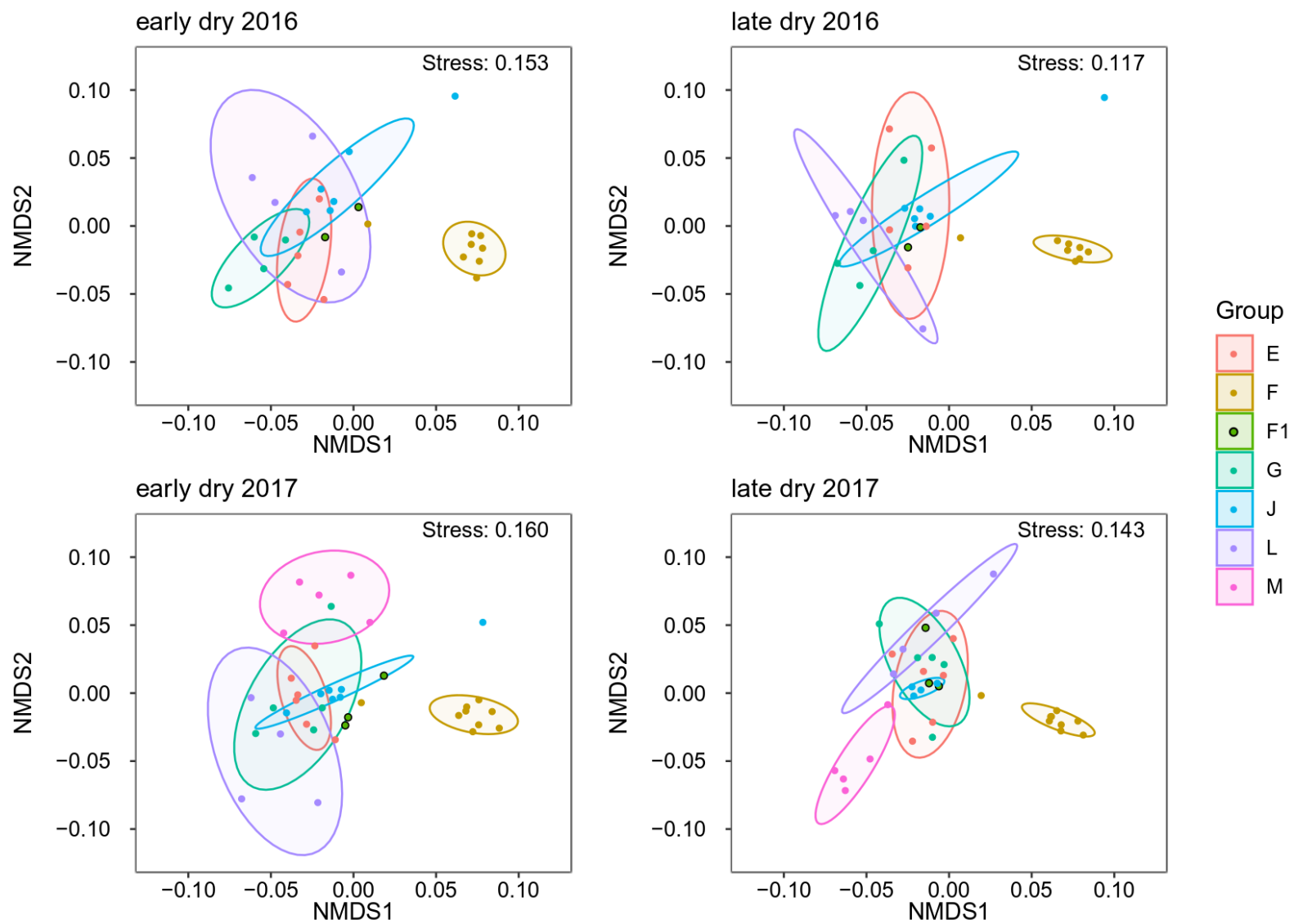

Figure 3 Nonmetric multidimensional scaling (NMDS) ordination of Verreaux's sifakas' gut bacterial composition data (GUniFrac distances) for each of the four field seasons. Data points represent individuals and colours indicate group membership. Ellipses indicate the $80 \%$ confidence ellipse for each group. For group F1, there were not enough data points to create ellipses, so we outlined the data points for better visibility. Several samples per individual per season were collected, however, here we only plotted one data point per animal, based on average dyadic beta dissimilarity per field season, to facilitate the overview. 


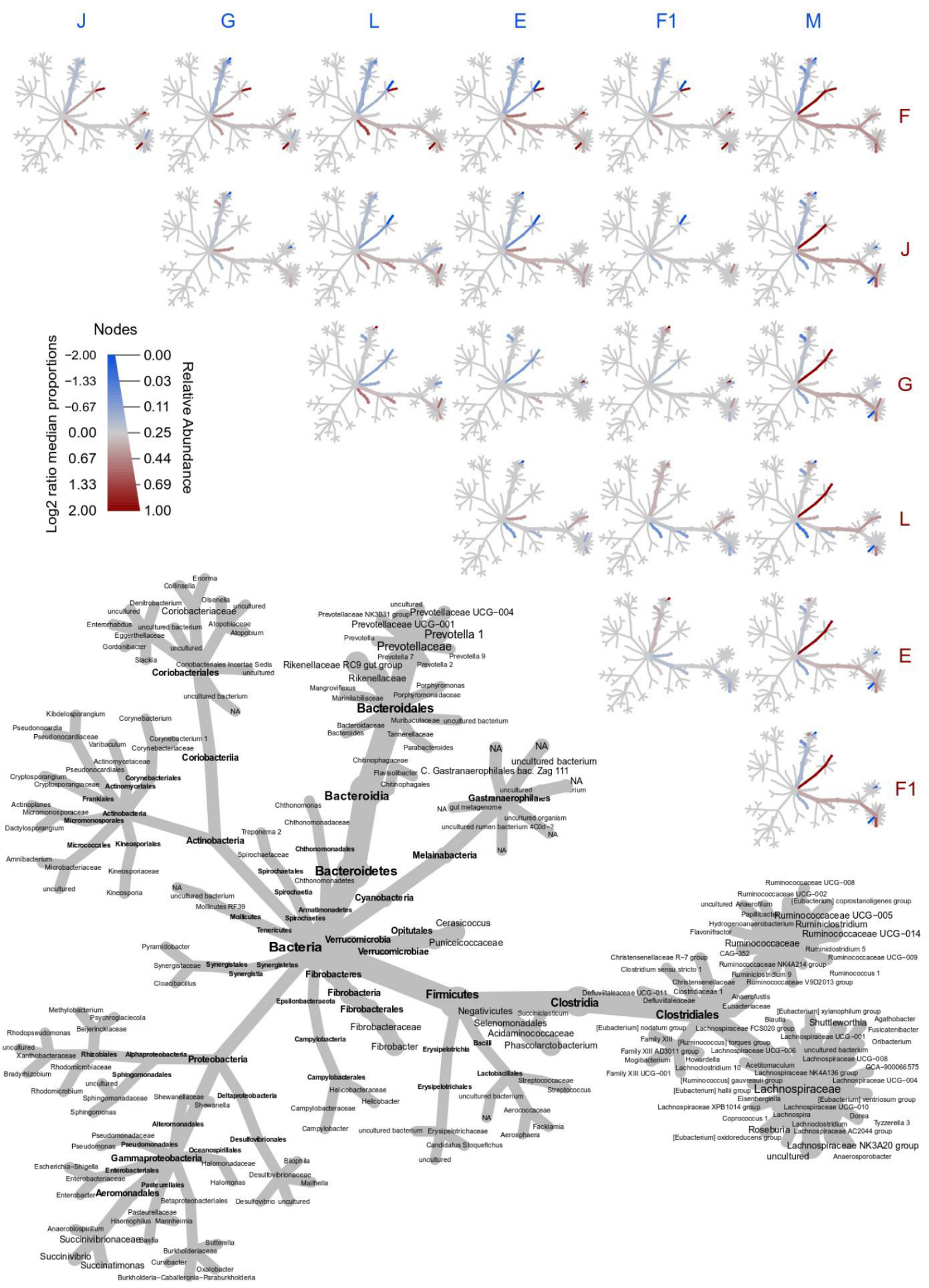

Figure 4 Differential heat tree matrix depicting between-group variation in gut microbial communities up to the genus level. The size of individual nodes within the grey cladogram depicts relative abundances of taxa identified at that taxonomic level. Smaller cladograms show pairwise comparisons between groups, based on Wilcoxon-signed rank tests: blue nodes indicate significantly higher abundances of the respective taxon in the group stated on the abscissa, than in the group stated on the ordinate. Red nodes indicate the opposite. 


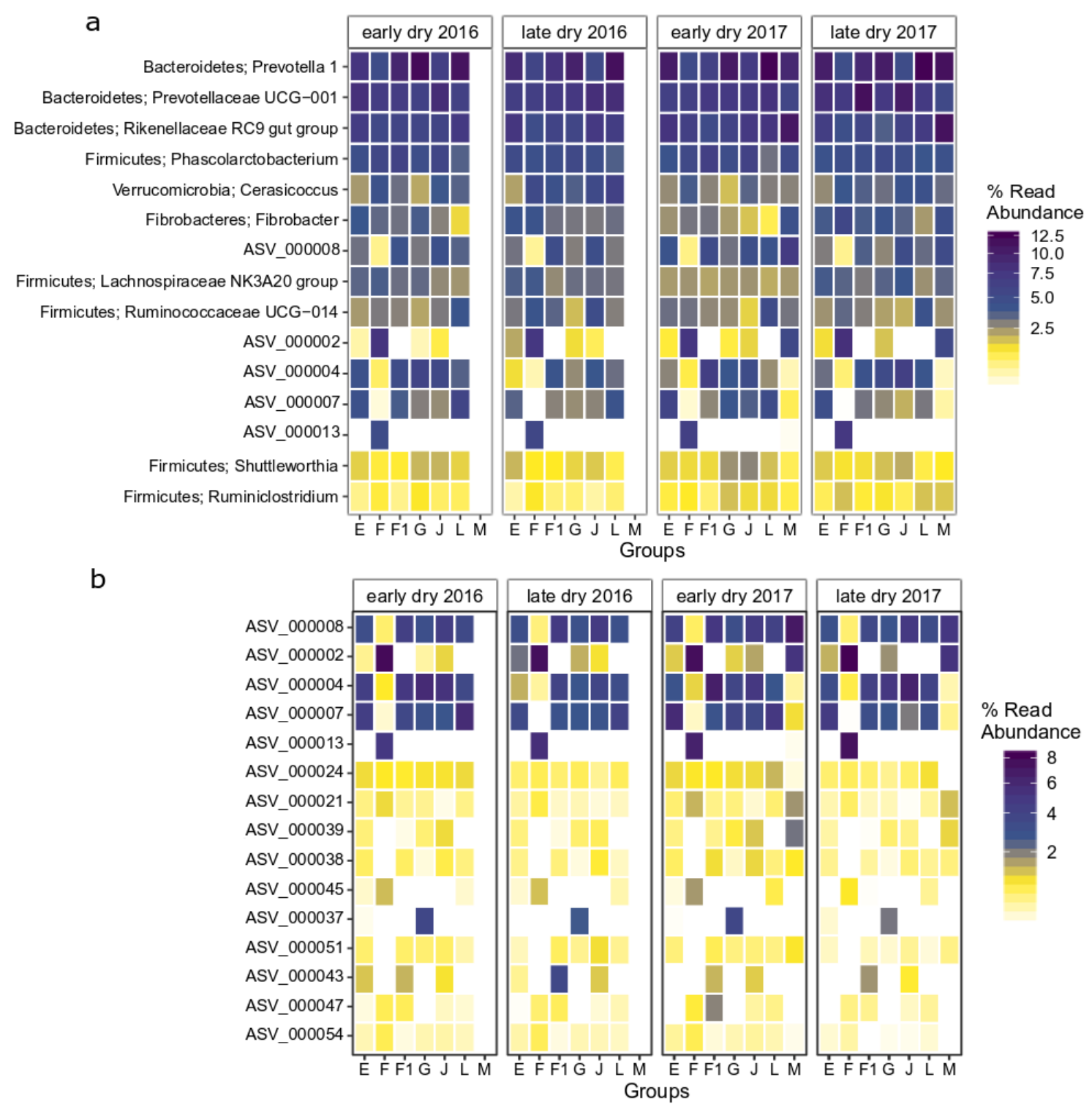

Figure 5 Heatmaps of the 15 most abundant a) genera and b) unassigned ASVs in Verreaux's sifakas' gut microbiomes

\section{Effects of body contact within groups}

The model examining effects of the time group members spent in body contact on dyadic GUniFrac dissimilarity was not significant $\left(\chi^{2}=0.223, d f=1, p=0.637, R_{m / c}^{2}=0.52 / 0.61\right.$ ) (Table S2, Figure $6 a$ ).

\section{Effects of maternal relatedness}

The model examining the effects of maternal relatedness on GUniFrac distances between individuals was highly significant $\left(\chi^{2}=164.418, \mathrm{df}=2, \mathrm{p}<0.001, \mathrm{R}_{\mathrm{m} / \mathrm{c}}=0.56 / 0.91\right)$ (Table S3). Maternal relatives share more similar microbiomes and there was a tendency for relatives living in the same group to share more similar microbiomes than relatives living in different groups (test of the interaction between maternal relatedness and group membership: $\left.\chi^{2}=3.679, \mathrm{df}=1, \mathrm{p}=0.055\right)$. Within groups, relatives shared more similar microbiomes than unrelated group members (Figure 7). 
a

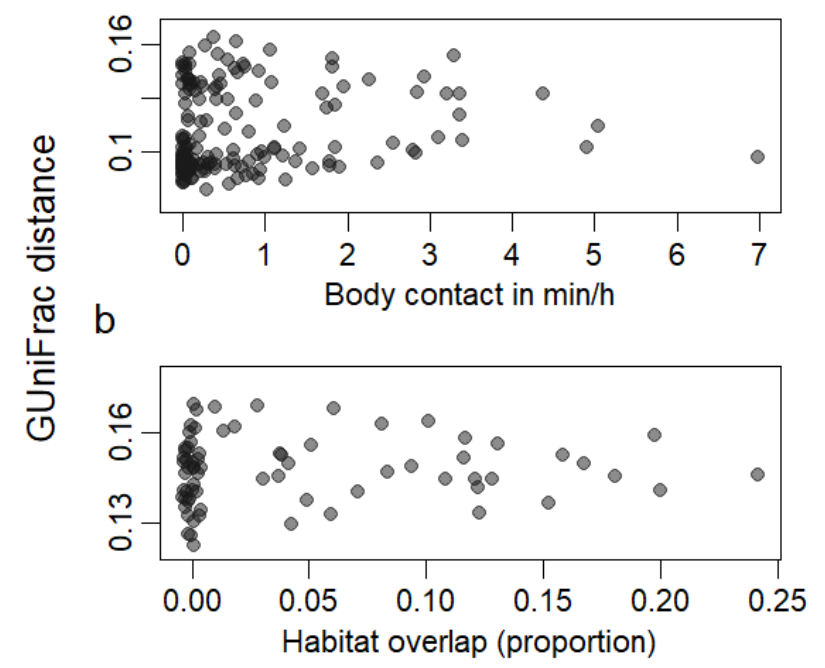

C

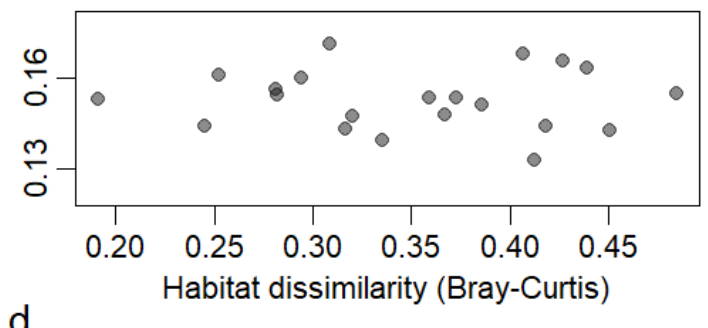

d

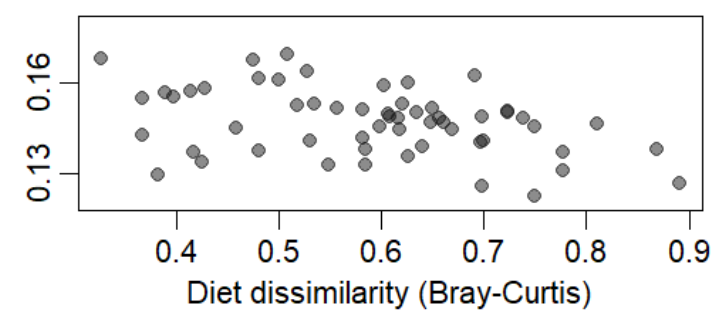

Figure 6 Influence of social contact within groups and differences in habitats and diets between groups on GUniFrac distances. a) Data points depict mean body contact rates per dyad per field season; $b+d$ ) Data points depict mean habitat overlaps and mean diet dissimilarities of group dyads per field season. c) Data points depict habitat dissimilarities of group dyads

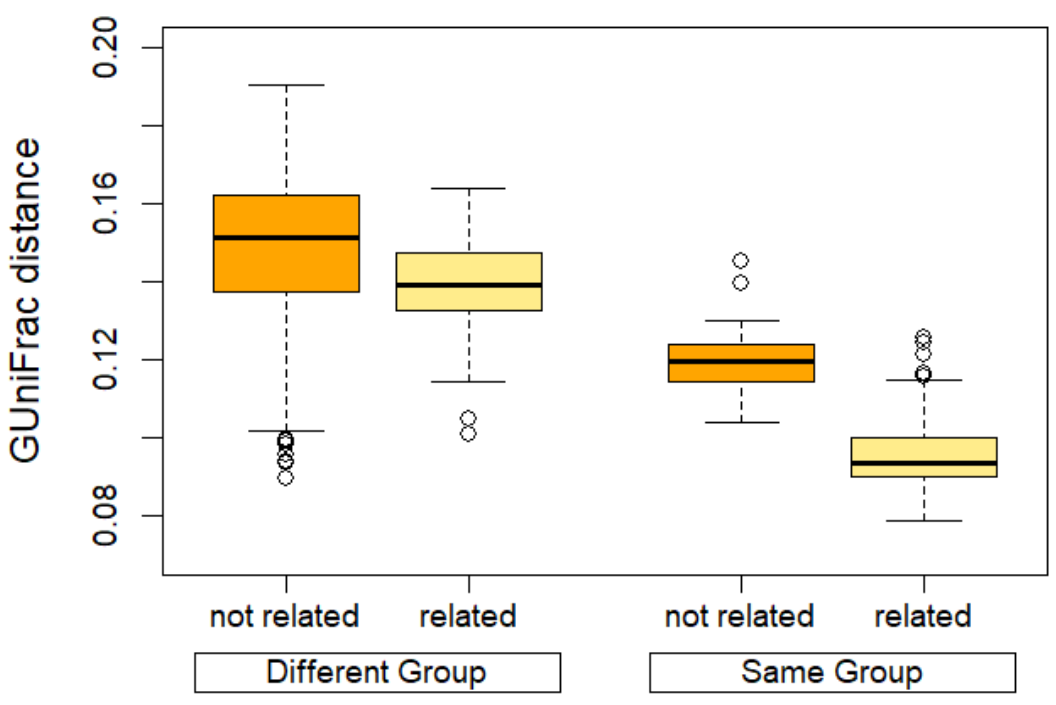

Figure 7 Influence of maternal relatedness on GUniFrac distances between individuals. Boxplots comprise dyads of all individuals with known maternal relationships. Lines indicating median, upper and lower quartiles. Whiskers indicate $+/-1.5$ interquartile ranges and small circles beyond whiskers indicate outliers

\section{Effects of habitat dissimilarity, habitat overlap and diet dissimilarity between groups}

Habitat dissimilarity and GUniFrac distances between groups were not correlated (Pearson: $r=-0.043$, $\mathrm{n}_{\text {Group dyads }}=21, \mathrm{p}=0.852$ ) (Figure $6 \mathrm{c}$ ). The models examining effects of habitat overlap and diet dissimilarities on groups' GUniFrac distances were not significant either (habitat overlap: $\chi^{2}=0.286, \mathrm{df}=$ $1, p=0.596, R_{m / c}^{2}=0.11 / 0.95$; diet dissimilarity: $\left.\chi^{2}=0.399, d f=1, p=0.528, R_{m / c}^{2}=0.09 / 0.95\right)$ (Tables S4 \& S5, Figure $6 b \& d)$. 


\section{Alpha diversity (PD)}

\section{Effects of group membership, season, study year and diet}

The model examining effects of group membership, season (early dry vs. late dry), study year and diet on individual PDs was significant $\left(\chi^{2}=113.650, \mathrm{df}=10, \mathrm{p}<0.001, \mathrm{R}_{\mathrm{m} / \mathrm{c}}=0.31 / 0.38\right)$ (Table S6). In detail, group membership and study year affected individual alpha diversity. Members of group $M$ had lower PDs than all groups except for group J. Group J had lower PDs than groups F, G and L (Table S7, Figure 8a). In 2017, all animals had lower PDs than in 2016 (Figure 8b). During the late dry season, PDs were slightly higher, while no correlations were found with individuals' fruit and leave intake rates (Figure 8c, $d \& e)$.

\section{Effects of group size}

The second model, examining effects of group size on PDs was not significant $\left(\chi^{2}=0.298, \mathrm{df}=1, p=0.858\right.$, $R_{m / c}^{2}=0.17 / 0.41$ ) (Table S8) (Figure $8 \mathrm{f}$ ). Body contact rates were not correlated to group size either (Pearson: $r=-0.078, n_{I D}=41, p=0.627$ ).
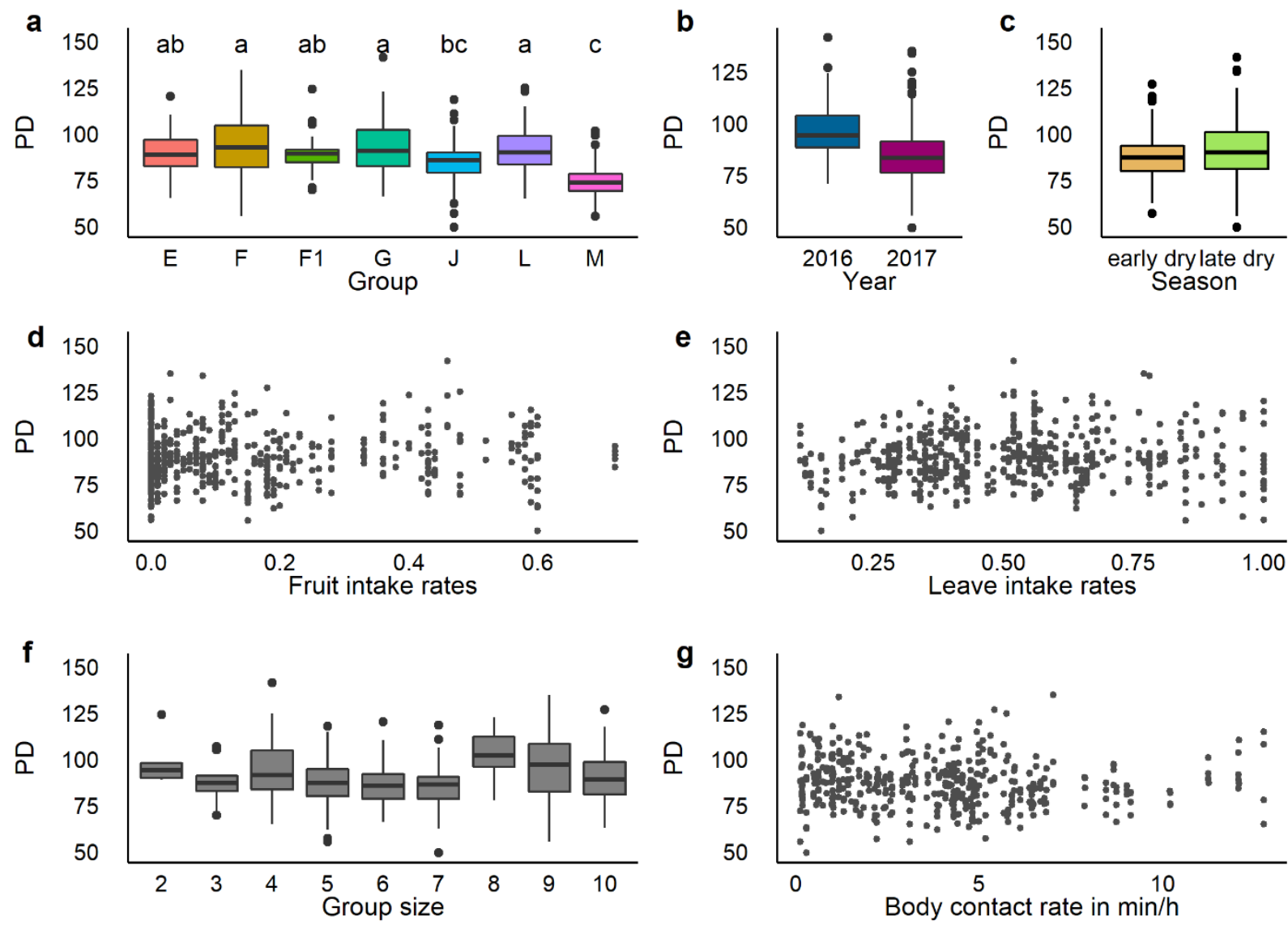

Figure 8 Influence of various factors on alpha diversity (Phylogenetic diversity). Coloured graphs indicate significant effects. a, b \& c) Influence of group identity, study year and season. Boxplots comprise all collected samples with lines indicate median, upper and lower quartiles. Whiskers indicate $+/-1.5$ interquartile ranges and small circles beyond whiskers indicate outliers. $d$ \& e) Influence of mean fruit and leave intake rates per field season. f) Influence of mean group size per field season. g) Influence of mean time spent in body contact per field season 
Effects of body contact within groups

The model examining effects of time spent in body contact within groups was not significant $\left(\chi^{2}=1.702\right.$, $\mathrm{df}=1, \mathrm{p}=0.192, \mathrm{R}_{\mathrm{m} / \mathrm{c}}^{2}=0.11 / 0.29$ ) (Table $\mathrm{s} 9$, Figure $8 \mathrm{~g}$ ).
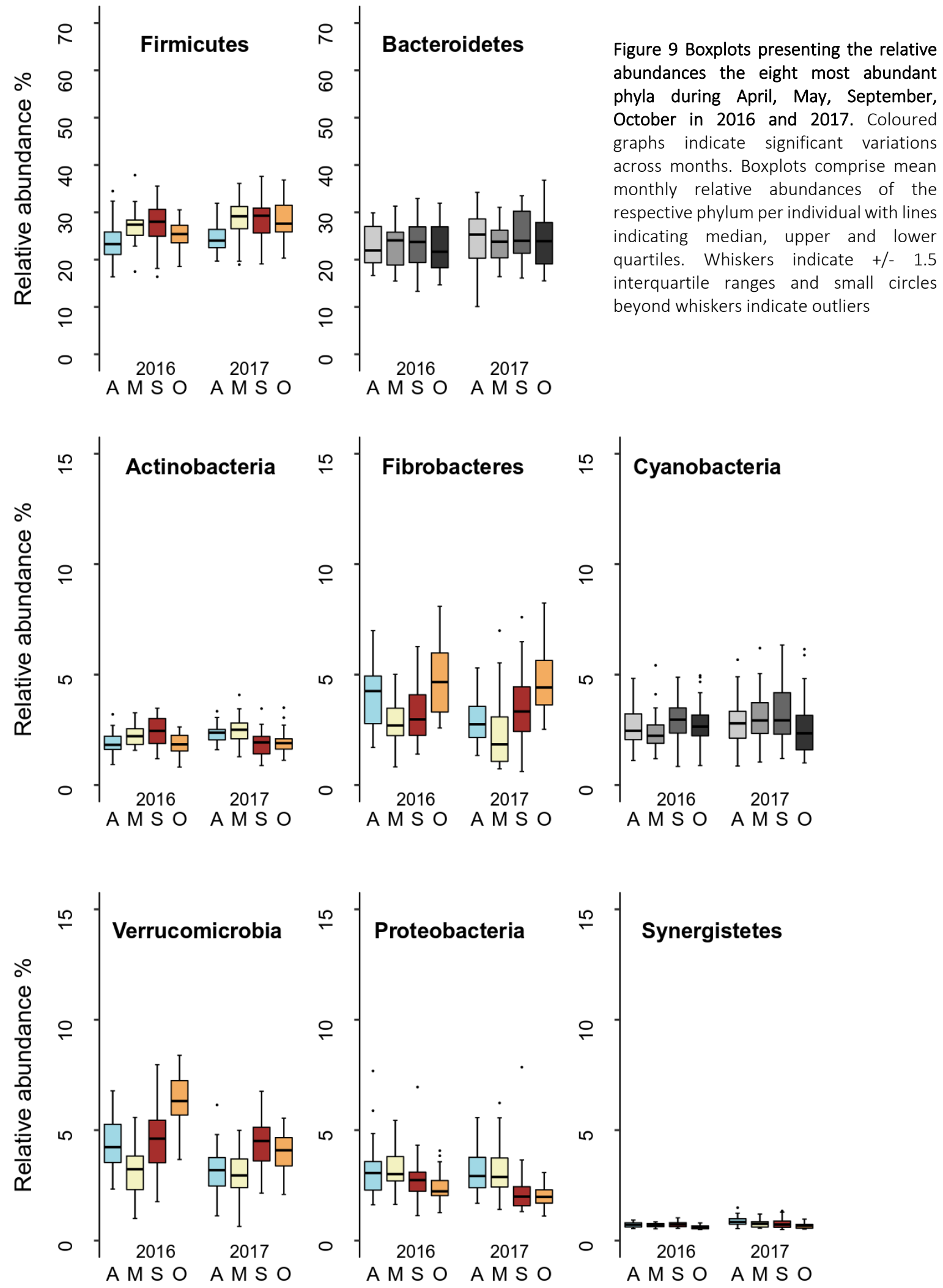


\section{Seasonal variation in relative abundances of core phyla}

We detected significant variations of monthly relative abundances in six out of eight core phyla in both study years (Tables S10 \& S11). The phyla Bacteroidetes and Cyanobacteria did not change in relative abundances with progressing dry season. Abundances of Firmicutes, Fibrobacteres and Verrumicrobia were significantly lower during the early dry season, while Proteobacteria showed opposite patterns (Figure 9). However, some of these effects varied with study year and were often more pronounced in 2017.

\section{Discussion}

We examined drivers of between-group variation in gut microbial communities of seven wild Verreaux's sifaka groups. Throughout all field seasons there was a consistent effect of group membership, as groups differed in both, composition and diversity of their gut microbiomes. Within groups, social contact neither predicted microbiome compositions nor bacterial diversity. Maternally related individuals shared more similar microbiomes, however, unrelated group members had more similar microbiomes than relatives living in different groups, indicating that the effects of group membership are stronger than the effects of kinship. None of the environmental predictors, i.e. habitat overlap, habitat dissimilarity and diet dissimilarity, explained variation of between-group gut microbiomes. Altogether, we conclude that environmental factors may determine the general arrangement for species- and population-specific gut microbial communities, whereas finer-scaled environmental differences between local groups might not have detectable effects. In contrast to most of the primate literature, we found kinship to play an important role for within-group microbial homogeneity. Moreover, social contact did not predict similarities in gut microbiomes within groups. Nevertheless, rare physical contact between individuals of different groups and frequent physical contact among group members remains a likely contributor to the here found group membership effects, however, more research is required to confirm this assumption.

\section{Sociality may drive variation in microbiome communities between but not within groups}

As expected, group membership predicted microbiome composition and diversity in our study species, confirming results of prior studies (Perofsky et al., 2017; Springer et al., 2017). In Verreaux's sifakas, group members regularly engage in grooming bouts and, like many lemur species (Eppley et al., 2017b; Morland, 1993; Ostner, 2002; Pereira et al., 1999), they use social thermoregulation, i.e. resting in body contact with conspecifics, especially during cold nights. In contrast, contact among individuals between groups is rare and most intergroup encounters proceed without any physical interactions (Benadi et al., 2008; Koch et al., 2016b). Therefore, increased physical contact among group members is a likely driver for greater within-group similarities in gut microbiomes. 
Within groups, however, variation in body contact between individuals did not further predict microbial similarity. This result contrasts findings of prior studies of chimpanzees and baboons (Moeller et al., 2016; Tung et al., 2015), but confirms findings in sooty mangabeys (Gogarten et al., 2018) and a different population of Verreaux's sifakas (Perofsky et al., 2017). In contrast to non-human primates, sifakas groom orally, which may facilitate bacterial transmission and sharing between individuals (Schmidt et al., 2019). However, on the other hand, Verreaux's sifakas also devote relatively little time to social activities (Richard, 1985) and grooming bouts are fairly short (Lewis, 2010). While proximity and grooming between group members shapes groups' distinct gut microbiomes, the generally low social interaction rates within groups may not further inflict dyadic microbial convergence.

Alpha diversity differed among groups, but this effect was not driven by variation in group size. As individuals in larger groups did not engage more frequently in social interactions either, which might increase social transmission of bacteria (Turnbull et al., 2011), this result is conclusive. In terms of between-group variation in alpha diversity, Grieneisen et al. (2017) propose a link between home range sizes and gut microbial diversity. Larger home ranges may harbour a larger variety of microbes which could, through direct transmission, increase animals' alpha diversity. Yet, differences in groups' home range sizes during the time of data collection, which are reported elsewhere (Rudolph et al., 2019), do not explain variation in alpha diversity in this study. For example, members of group $M$, which had significantly lower bacterial diversities than most of the other study groups, occupied the largest home range, whereas members of group J had similar bacterial diversities in relation to the other groups despite occupying the smallest home range.

Increased glucocorticoid concentrations (GCS) can result in a loss of microbial diversity and richness, as shown in studies in lab mice and barn swallows (Hirundo rustica erythrogaster) (Bailey et al., 2011; Levin et al., 2016). Similarly, parasite infections can alter microbiome composition and diversity (Morton et al., 2015; Zaiss and Harris, 2016). However, our study groups did not differ in GCs or helminth prevalence during the study period (Rudolph et al., 2019), ruling out potential effects of GCs and helminths on group-level alpha diversity. Variation in consumed plant species and differences in habitat features are also likely to alter alpha diversities between groups (Clayton et al., 2018; Reese and Dunn, 2018), yet, here we could not detect any links. Thus, the drivers for between-group variation in bacterial diversities remain unidentified in this study.

Individuals that spent more time in body contact with group members did not harbour more diverse microbiomes. Other studies examining effects of affiliation on alpha diversity reported various patterns. In line with our findings, no correlation was found in baboons (Grieneisen et al., 2017), while negative correlations were reported in red-bellied lemurs (Raulo et al., 2017). Positive correlations were found in chimpanzees, howler monkeys (Amato et al., 2017b; Moeller et al., 2016) and in a different population of Verreaux's sifakas (Perofsky et al., 2017). The findings of Perofsky et al. (2017) are based 
on grooming network metrics, whereas we used dyadic interaction rates. After replicating some of the statistical analyses of Perofksy et al. (2017), we concluded that our data are insufficient in density for conducting reliable social network analyses and refrained from inferring meaningful conclusions from these analyses (see Appendix Chapter 4). Thus, it is possible that differences in observation and statistical methods account for the different results found between Perofsky et al. (2017) and our study. However, it may also be that variation in environmental conditions affects animals' social interaction patterns in different populations. Altogether, the impact of social contact on alpha diversity seems to vary within and between species. Differences in social patterns may explain these variations (Kuthyar et al., 2019), yet, more research in more species is required to understand the mechanisms.

Direct physical contact constitutes one important mechanism for microbial transmission. However, there remains a lack of groundwork for identifying the routes and conditions of these transmissions (Brito et al., 2019; Kuthyar et al., 2019; Robinson et al., 2019). The intensity of microbial transmissions could be impacted by the duration of social interactions, but may additionally depend on bacteria's viability under external environmental conditions, for example when exposed to certain temperatures or ultraviolet radiation (Browne et al., 2017; Ferguson and Signoretto, 2011). Less direct pathways can also contribute to microbial transmissions. For example, humans can emit "microbial clouds", i.e. airborne microbes, which may colonize other humans (Meadow et al., 2015) but usually comprise mainly of skin-associated bacteria (Browne et al., 2017). Surfaces that came in contact with human or animal hosts can harbour, amongst others, high proportions of intestinal-associated bacteria which have the potential to transmit to other hosts (Browne et al., 2017; Song et al., 2013). Thus, proximity alone may be sufficient for causing microbial convergence in cohabitating individuals without further ado of direct social contact. Altogether, research on the social transmission of microbes remains in its infancy and more research is necessary to explain the various patterns found across and within taxa.

\section{Maternally related individuals share more similar microbiomes}

In line with studies on red squirrels (Tamiasciurus hudsonicus), cheetahs (Acinonyx jubatus) and gopher tortoises (Gopherus polyphemus) (Ren et al., 2017; Wasimuddin et al., 2017; Yuan et al., 2015), maternally related individuals shared more similar microbiomes than maternally unrelated individuals, even after controlling for group membership. This result contrasts with findings in studies of primates, where either no or only weak links with kinship were found (Degnan et al., 2012b; Goodfellow et al., 2019; Grieneisen et al., 2017; Tung et al., 2015). Over a course of a lifetime, social interactions can crucially shape individuals' gut microbiomes (Björk et al., 2019). In species spending large proportions of their times engaging in social activities, like chimpanzees or baboons, majority of microbial communities might thus be acquired via social transmission, potentially replacing maternally inherited phylotypes (Moeller et al., 2016; Ren et al., 2017). In species with fewer social interactions, however, maternal effects on gut 
microbiomes might last into adulthood, which would explain our findings. The effects of maternal relatedness on Verreaux's sifakas' microbiomes likely contributed to the effects of group membership, as the majority of maternally related individuals lived within the same group. However, since maternally unrelated group members shared more similar microbiomes than relatives living in different groups, genetic relatedness is clearly not the only driver for between-group variation in microbiome compositions in this species.

\section{Neither habitat nor diet explain between-group variation in microbial communities}

Our study site is very heterogenous in terms of forest structure and composition (Kappeler and Fichtel, 2012; Sorg and Rohner, 1996) and sifakas' home ranges differ in both, food tree richness and abundances (Rudolph et al., 2019). However, neither home range similarity nor home range overlap reflected microbiome similarities between groups. For the latter, Perofsky et al. (2017) found similar patterns in another population of Verreaux's sifakas. In contrast, various studies in other primates and fish reported links between variation in habitat type and microbiome composition or diversity (Amato et al., 2015; Barelli et al., 2015; Bennett et al., 2016b; Björk et al., 2019; Greene et al., 2019b; Smith et al., 2015). However, these studies compared groups or populations which either lived in greater distance to each other and/or inhabited highly different landscapes, e.g. disturbed vs. undisturbed forests or lakes vs. streams, whereas we compared neighbouring groups living within an area of $1 \mathrm{~km}^{2}$ of the same habitat type. Thus, the comparatively minor variations in habitat features between our study groups do not seem to predict microbiome variations.

Groups did not vary in their leave and fruit intakes across seasons (see Appendix Chapter 4), but some groups consumed more similar plant species than others. However, more similar diets did not predict microbiome similarities and the amounts of fruits or leaves in the diet were not related to animals' gut microbial diversity. The latter result is consistent with findings of a prior study in the same population (Springer et al., 2017). Sifakas' gut microbiomes clearly reflect their frugi-folivorous diet as they are dominated by polysaccharide-fermenting taxa like Lachnospiracea, Ruminococcocaceae (Phylum Firmicutes) and Prevotellaceae (Phylum Bacteroidetes) (Amato et al., 2019; Greene et al., 2018, 2019b). Yet, while animals' foraging strategies may determine their basic gut microbial composition and diversity (Greene et al., 2018, 2019b), it appears that minor shifts in dietary patterns do not inflict detectable changes and are therefore unlikely drivers of between-group variation in gut microbiomes in this species.

\section{Seasonal effects on microbial communities differ between years}

Verreaux's sifakas' phylogenetic diversity was weakly linked to seasonality and most core phyla showed seasonal variation in relative abundances, reflecting dietary shifts of fruit and leave consumptions during the dry season. Our results are mainly in line with those of a prior study in the same population. Springer et al. (2017) reported clear seasonal variation of Firmicutes-Bacteroidetes ratios, whereas we did not 
detect any seasonal changes in Bacteroidetes. With progression of the dry season, general food availability decreases and sifakas increase their intake of other plant parts, like barks, stems and flowers, leading to an increased fibre intake (Koch et al., 2017; Norscia et al., 2006) and thus a higher abundance of fibre-digesting Firmicutes (Flint et al., 2012; Gomez et al., 2016; Martens et al., 2014). Decreases in fruit intake are expected to result in lower abundances of Bacteroidetes (Gomez et al., 2016). However, the increase in consumption of various other plant parts during the late dry season might maintain the abundance of Bacteroidetes, which can digest a very broad array of mostly, but not exclusively, soluble substrates (Greene et al., 2019a; Martens et al., 2014; Wu et al., 2011). We believe that the contrasting patterns found in the study of Springer et al. (2017) and the present one might reflect annual variation in food availability and diet. In fact, we found monthly differences in phyla abundances to vary between years and animals had lower gut microbial diversities in 2017 than in 2016.

\section{The missing puzzle pieces}

Notably, about $30 \%$ of amplicon sequences belonging to $50 \%$ of all ASVs could not be assigned to any taxonomy, confirming results of prior studies in lemurs (Amato et al., 2019; Greene et al., 2019b, 2019a; Perofsky et al., 2019, 2017; Springer et al., 2017). This high proportion of unknown sequences is especially prevalent in members of the Indriidae family. The little microbial characterisations of taxa from Madagascar together with indriids' folivorous diets and complex gastrointestinal tracts are likely causes for the lack of taxonomic assignments. Interestingly, some of the unassigned taxa were high in abundance in some groups, yet, nearly completely absent in others (Figures 5b). This pattern implies that unclassified ASVs contribute to the divergence between our study groups' microbial communities. However, until these taxa are characterized, we cannot further explore this effect.

\section{Conclusions}

With this study, we contribute to the understanding of the relative importance of 1) environmental, 2) intrinsic, and 3) social factors on shaping gut microbial communities in wild animals. Our findings indicate that 1) environmental factors likely define the general set-up of species- or population-specific gut microbiota, while minor differences in microhabitat features or diets among local groups do not seem to inflict detectable variation. 2) Kinship plays an important role for the development of individual microbial patterns, however, whether these effects derived from increased proximity and interactions between kin or from genetic inheritance remains to be studied. Importantly, kinship effects seem to depend on species-specific frequencies of social contact, as social transmission can potentially overwrite microbiota acquired via kin. 3) Life in permanent social groups can promote the convergence of gut microbial communities. However, variation in species' social systems affects microbial dispersal opportunities, which is why the degree to which social interactions affect individual gut microbiota may vary across 
species. More comprehensive studies across species with varying social systems will therefore help to shed more light on the co-evolutionary dynamics that shaped host-microbiota relationships.

\section{Acknowledgments}

We are grateful to the Malagasy Ministère de l'Environnement, the Departement de Biologie Ecologie et Conservation Animale de l'Université d'Antananarivo and the Centre National de Formation, d'Etudes et de Recherche en Environnementet Foresterie de Morondava for supporting our research in Kirindy Forest. We also thank Mamy Razafindrasamba and Tiana Andrianjanahary for their help in data collection, Dr Roger Mundry for statistical advice, Virgile Manin and the other members of the DFG research unit "Sociality and Health in Primates" (FOR 2136) for their precious remarks and constructive discussions. This research was funded by the Deutsche Forschungsgemeinschaft (DFG), grant number Ka 1082/29-2, awarded to PMK.

\section{Ethical approval}

Statement of ethical approval, approval of research protocols and capture procedures were approved by a committee of the Ministry for the Environment, Water and Forests of Madagascar (MINEEF). All procedures performed in studies involving animals were in accordance with the ethical standards of the institution or practice at which the studies were conducted. This article does not contain any studies with human participants performed by any of the authors.

\section{Author contributions}

PMK, CF, and KR designed the study. KR performed data collection and conducted the lab work. KR and DS and analysed the data. KR drafted the manuscript and all authors contributed to writing and revising of the manuscript. 


\section{Appendix: Chapter 4}

This supplementary file contains more detailed information on:

d. Supplemental Methods

e. Supplemental Results

p. 114

f. Further Analyses..

p. 122

a. Beta diversity: Bray-Curtis dissimilarity.

p. 122

b. Alpha diversity: Shannon Index

p. 126

c. Comparisons with Perofsky et al. (2017): Social network statistics and microbiomes........

p. 129

d. Leave and fruit intake per group per season

p. 133

\section{(1) Supplemental Methods}

\section{Home range overlap}

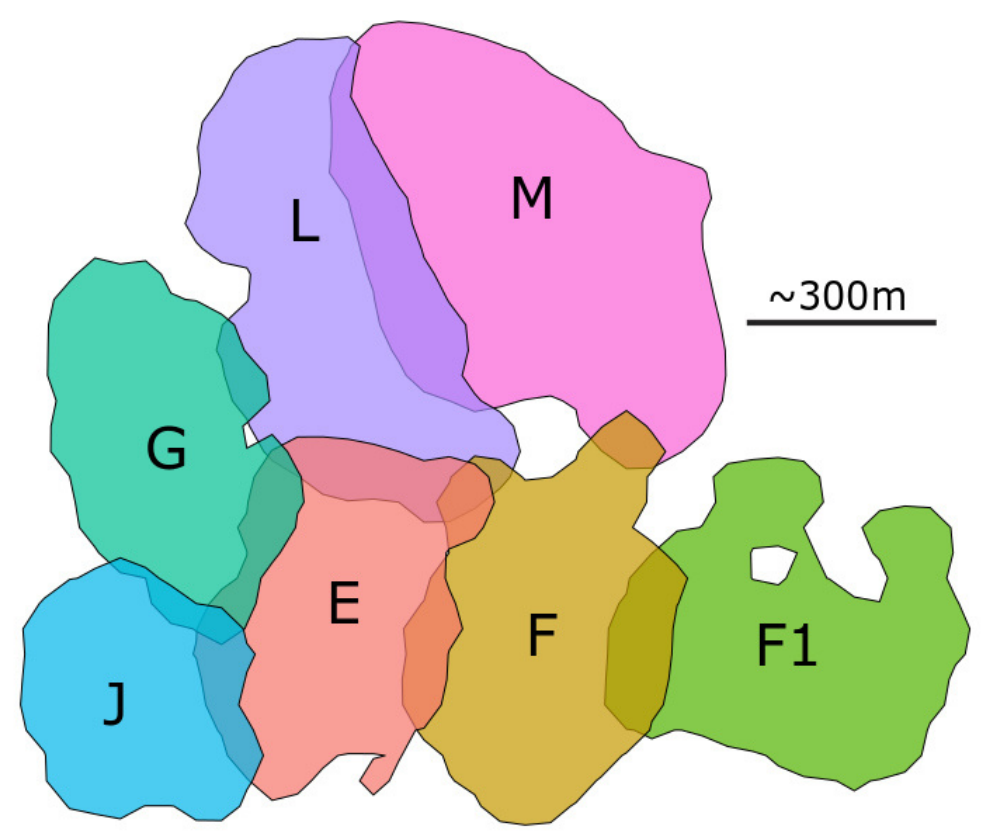

Figure S1 Illustration of home range locations and overlaps of all study groups. Areas indicate average 95\% Kernels over the complete study period. 
(2) Supplemental Results

Beta diversity (GUniFracs)

Within- and between-individual differences on microbiome compositions

Table S1 Comparing GUniFrac distances within and between group members in seven groups of Verreaux's sifakas; results of the full model (GLMM; Nobservations $=455, N_{1 D}$ dyads $\left.=153\right)$.

\begin{tabular}{|c|c|c|c|c|c|c|c|c|c|}
\hline Term & Est & SE & Lower $\mathrm{Cl}$ & Upper Cl & Min $^{a}$ & $\operatorname{Max}^{a}$ & $\chi^{2 b}$ & $d f$ & $P$ \\
\hline (Intercept) & 0.123 & 0.004 & 0.114 & 0.131 & 0.119 & 0.126 & c & c & c \\
\hline Same ID (yes) ${ }^{d}$ & -0.019 & 0.003 & -0.025 & -0.011 & -0.021 & -0.017 & 26.366 & 1 & $<0.001$ \\
\hline Season ${ }^{\mathrm{e}}$ & & & & & & & $11.861^{f}$ & $3^{f}$ & $0.008^{f}$ \\
\hline late dry 2017 & -0.006 & 0.002 & -0.010 & -0.002 & -0.008 & -0.005 & c & c & c \\
\hline early dry 2016 & 0.000 & 0.002 & -0.003 & 0.004 & -0.002 & 0.002 & c & c & c \\
\hline early dry 2017 & -0.005 & 0.001 & -0.008 & -0.003 & -0.006 & -0.005 & c & c & c \\
\hline
\end{tabular}

${ }^{a}$ Minimum and maximum of model estimates obtained when dropping levels of random effects one at a time

${ }^{b}$ Results of a likelihood ratio test comparing the full model with a reduced model lacking the respective term

c Not shown as not having a meaningful interpretation. For intercepts, p-values would refer to estimated fGCM concentrations, when all covariates are at zero. For main effects of predictors which are involved in interaction terms, $p$-values refer only to the effect of that involved predictor with the interacting covariate at zero. This means the main effects of predictors involved in interactions depend on the value of the other main effects and are, therefore, not interpretable in themselves. Therefore, we consider $P$ values of main effects to be meaningful only when the predictors are not involved in an interaction.

"Manually dummy-coded with the season "no" being the reference category

e Manually dummy-coded with the season "late dry 2016" being the reference category

${ }^{f}$ Values refer to the overall test of the effect of the predictor ( "Season"), not the specific level indicated in the respective row

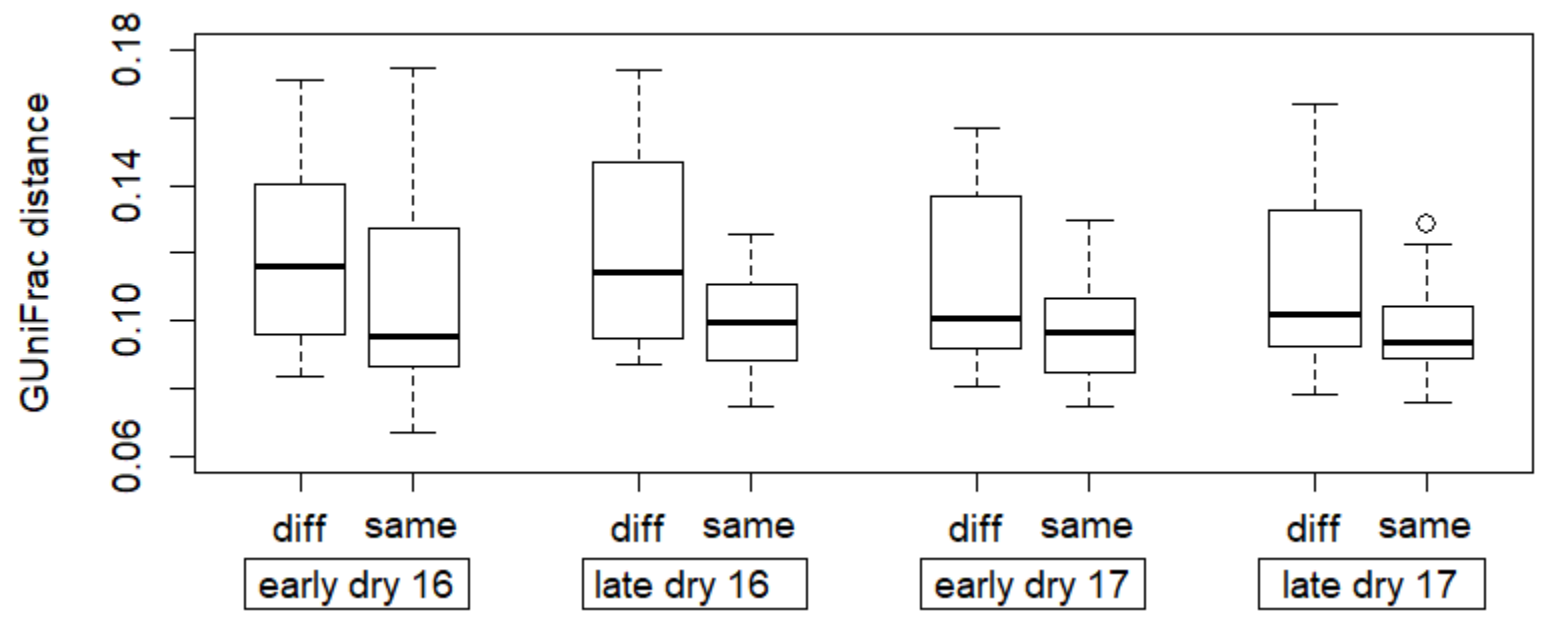

Figure S2 Comparison of microbiome dissimilarities between samples collected from the same individual and samples collected from different individuals living in the same group during four different field seasons. Lines indicate median, upper and lower quartiles. Whiskers indicate +/- 1.5 interquartile ranges and small circles beyond whiskers indicate outliers. 


\section{Effects of body contact within groups (GUniFrac)}

Table S2 Influence of body contact on GUniFrac distances (sqrt-transformed) among group member of seven groups of Verreaux's sifakas; results of the full model $\left(G L M M ; N_{\text {Observations }}=2856, N_{I D}\right.$ dyads $\left.=86\right)$.

\begin{tabular}{|c|c|c|c|c|c|c|c|c|c|}
\hline Term & Est & SE & Lower Cl & Upper Cl & $\operatorname{Min}^{a}$ & $\mathrm{Max}^{\mathrm{a}}$ & $\chi^{2 b}$ & $d f$ & $P$ \\
\hline (Intercept) & 0.378 & 0.003 & 0.372 & 0.384 & 0.376 & 0.380 & c & c & c \\
\hline Body contact ${ }^{d}$ & 0.001 & 0.001 & -0.001 & 0.003 & -0.001 & 0.001 & 0.223 & 1 & 0.637 \\
\hline Related (yes) & -0.062 & 0.003 & -0.069 & -0.056 & -0.065 & -0.055 & 132.560 & 1 & $<0.001$ \\
\hline Season ${ }^{f}$ & & & & & & & $14.317^{g}$ & $2^{g}$ & $<0.001^{\mathrm{g}}$ \\
\hline late dry 2017 & -0.011 & 0.003 & -0.018 & -0.003 & -0.014 & -0.007 & c & c & c \\
\hline early dry 2017 & -0.008 & 0.002 & -0.012 & -0.005 & -0.010 & -0.007 & c & c & c \\
\hline
\end{tabular}

a Minimum and maximum of model estimates obtained when dropping levels of random effects one at a time

${ }^{b}$ Results of a likelihood ratio test comparing the full model with a reduced model lacking the respective term

c Not shown as not having a meaningful interpretation. See footnotes of Table A.1 for details.

${ }^{\mathrm{d}} \mathrm{z}$-transformed, mean and SD of the original values were 0.771 and $1.187(\mathrm{~min} / \mathrm{h})$, respectively

"Manually dummy-coded with the season "no" being the reference category

${ }^{f}$ Manually dummy-coded with the season "late dry 2016" being the reference category

gValues refer to the overall test of the effect of the predictor ("Season"), not the specific level indicated in the respective row

\section{Effects of maternal relatedness (GUniFrac)}

Table S3 Influence of maternal relatedness on GUniFrac distances in seven groups of Verreaux's sifakas; results of the full model (GLMM; Nobservations $=1439, \mathrm{~N}_{\mathrm{ID}}$ dyads $\left.=502\right)$.

\begin{tabular}{|c|c|c|c|c|c|c|c|c|c|}
\hline Term & Est & SE & Lower $\mathrm{Cl}$ & Upper Cl & $\operatorname{Min}^{a}$ & $\operatorname{Max}^{a}$ & $\chi^{2 b}$ & $d f$ & $P$ \\
\hline (Intercept) & 0.154 & 0.001 & 0.152 & 0.156 & 0.154 & 0.154 & c & c & c \\
\hline Related (yes) ${ }^{\mathrm{d}}$ & -0.030 & 0.006 & -0.042 & -0.018 & -0.031 & -0.029 & c & c & c \\
\hline Same group (yes) & -0.011 & 0.003 & -0.016 & -0.005 & -0.011 & -0.010 & c & c & c \\
\hline Season ${ }^{f}$ & & & & & & & $257.245^{\mathrm{g}}$ & $3^{g}$ & $<0.001^{g}$ \\
\hline late dry 2017 & -0.008 & 0.001 & -0.009 & -0.006 & -0.008 & -0.008 & c & c & c \\
\hline early dry 2016 & 0.002 & 0.001 & 0.001 & 0.003 & 0.002 & 0.002 & c & c & c \\
\hline early dry 2017 & -0.007 & 0.001 & -0.008 & -0.006 & -0.007 & -0.007 & c & c & c \\
\hline $\begin{array}{l}\text { Related (yes) }{ }^{*} \\
\text { Same group (yes) }\end{array}$ & -0.013 & 0.007 & -0.027 & 0.000 & -0.015 & -0.012 & $3.679^{h}$ & $2^{h}$ & $0.055^{h}$ \\
\hline $\begin{array}{l}\text { a Minimum and maximu } \\
\text { b Results of a likelihood } \\
{ }^{\mathrm{c}} \text { Not shown as not havin } \\
{ }^{\mathrm{d}} \text { Manually dummy-code } \\
{ }^{\mathrm{e}} \text { Manually dummy-code } \\
{ }^{\mathrm{f}} \text { Manually dummy-code } \\
{ }^{\mathrm{g}} \text { Values refer to the ove } \\
{ }^{\mathrm{h}} \text { Values refer to the ove }\end{array}$ & $\begin{array}{l}\text { odel estima } \\
\text { st comparir } \\
\text { aningful int } \\
\text { the season } \\
\text { the season } \\
\text { he season } \\
\text { of the effe } \\
\text { of the inte }\end{array}$ & $\begin{array}{l}\text { es obtain } \\
\text { s the full } n \\
\text { rpretatior } \\
\text { no" being } \\
\text { no" being } \\
\text { late dry } 2 \\
\text { t of the p } \\
\text { action "Re }\end{array}$ & $\begin{array}{l}\text { d when dropp } \\
\text { odel with a re } \\
\text { See footnote } \\
\text { che reference } \\
\text { che reference } \\
16 \text { " being the } \\
\text { edictor ("Seasc } \\
\text { ated*Same Gr }\end{array}$ & $\begin{array}{l}\text { gg levels of ra } \\
\text { uced model I } \\
\text { of Table A.1 } \\
\text { ategory } \\
\text { ategory } \\
\text { eference cate } \\
\text { n") }^{\prime \prime} \\
\text { op", not the }\end{array}$ & $\begin{array}{l}\text { dom effect } \\
\text { king the re } \\
\text { r details. }\end{array}$ & one at a ti & e respectiv & & \\
\hline
\end{tabular}




\section{Effects of habitat overlap between groups (GUniFrac)}

Table S4 Influence of habitat overlap on GUniFrac distances (In-transformed) among seven groups of Verreaux's sifakas; results of the full model (GLMM; Nobservations $=72, N_{\text {Group dyads }}=21$ ).

\begin{tabular}{|c|c|c|c|c|c|c|c|c|c|}
\hline Term & Est & SE & Lower Cl & Upper Cl & $\operatorname{Min}^{a}$ & $\operatorname{Max}^{a}$ & $\chi^{2 b}$ & $d f$ & $P$ \\
\hline (Intercept) & -1.876 & 0.016 & -1.910 & -1.844 & -1.883 & -1.866 & c & c & c \\
\hline Overlap ${ }^{d}$ & 0.004 & 0.006 & -0.009 & 0.016 & 0.001 & 0.010 & 0.286 & 1 & 0.593 \\
\hline Season ${ }^{\mathrm{e}}$ & & & & & & & $42.066^{f}$ & $3^{f}$ & $<0.001^{f}$ \\
\hline late dry 2017 & -0.057 & 0.007 & -0.071 & -0.043 & -0.060 & -0.054 & c & c & c \\
\hline early dry 2016 & 0.000 & 0.008 & -0.017 & 0.017 & -0.003 & 0.005 & c & c & c \\
\hline early dry 2017 & -0.046 & 0.008 & -0.062 & -0.030 & -0.049 & -0.043 & c & c & c \\
\hline \multicolumn{10}{|c|}{$\begin{array}{l}\text { "Minimum and maximum of model estimates obtained when dropping levels of random effects one at a time } \\
\text { b Results of a likelihood ratio test comparing the full model with a reduced model lacking the respective term } \\
\text { "Not shown as not having a meaningful interpretation. See footnotes of Table A.1 for details. } \\
\text { d z-transformed, mean and SD of the original values were } 0.045 \text { and } 0.063 \text {, respectively } \\
\text { e Manually dummy-coded with the season "late dry } 2016 \text { " being the reference category } \\
\text { f Values refer to the overall test of the effect of the predictor ("Season") not the specific level indicated in the }\end{array}$} \\
\hline
\end{tabular}

\section{Effects of diet dissimilarity between groups (GUniFrac)}

Table S5 Influence of diet dissimilarity (Bray-Curtis) on GUniFrac distances (In-transformed) among seven groups of Verreaux's sifakas; results of the full model (GLMM; $\left.N_{\text {Observations }}=57, \mathrm{~N}_{\text {Group dyads }}=21\right)$.

\begin{tabular}{|c|c|c|c|c|c|c|c|c|c|}
\hline Term & Est & SE & Lower $\mathrm{Cl}$ & Upper Cl & $\operatorname{Min}^{a}$ & $\operatorname{Max}^{a}$ & $\chi^{2 b}$ & $d f$ & $P$ \\
\hline (Intercept) & -1.882 & 0.016 & -1.913 & -1.851 & -1.888 & -1.871 & c & $\mathrm{c}$ & $\mathrm{c}$ \\
\hline Diet dissimiliarity $^{d}$ & -0.004 & 0.006 & -0.017 & 0.009 & -0.007 & -0.001 & 0.399 & 1 & 0.528 \\
\hline Season $^{e}$ & & & & & & & $34.162^{f}$ & $2^{f}$ & $<0.001^{f}$ \\
\hline late dry 2017 & -0.059 & 0.007 & -0.072 & -0.045 & -0.062 & -0.055 & c & c & c \\
\hline early dry 2017 & -0.038 & 0.008 & -0.054 & -0.022 & -0.044 & -0.034 & c & c & c \\
\hline
\end{tabular}

${ }^{a}$ Minimum and maximum of model estimates obtained when dropping levels of random effects one at a time

${ }^{b}$ Results of a likelihood ratio test comparing the full model with a reduced model lacking the respective term

c Not shown as not having a meaningful interpretation. See footnotes of Table A.1 for details.

dz-transformed, mean and SD of the original values were 0.592 and 0.132 , respectively

"Manually dummy-coded with the season "late dry 2016" being the reference category

${ }^{\text {fV}}$ Values refer to the overall test of the effect of the predictor ("Season"), not the specific level indicated in the respective row 


\section{Alpha diversity (Phylogenetic diversity)}

Effects of group membership, season, study year and diet

Table S6 Influence of various factors on PDs (In-transformed) in seven groups of Verreaux's sifakas; results of the full model $\left(\right.$ GLMM; Nobservations $\left.=519, N_{I D}=41, N_{\text {Group }}=7\right)$.

\begin{tabular}{|c|c|c|c|c|c|c|c|c|c|c|}
\hline Term & & Est & SE & Lower $\mathrm{Cl}$ & Upper Cl & $\operatorname{Min}^{a}$ & $\operatorname{Max}^{a}$ & $\chi^{2 b}$ & $d f$ & $P$ \\
\hline (Intercept) & & 4.521 & 0.020 & 4.483 & 4.560 & 4.508 & 4.575 & c & c & c \\
\hline \multirow[t]{6}{*}{ Group $^{\mathrm{e}}$} & $\mathrm{F}$ & 0.044 & 0.021 & 0.006 & 0.088 & 0.034 & 0.060 & $22.056^{d}$ & $6^{d}$ & $0.001^{d}$ \\
\hline & F1 & 0.000 & 0.027 & -0.051 & 0.051 & -0.040 & 0.028 & c & c & c \\
\hline & G & 0.035 & 0.023 & -0.011 & 0.083 & -0.008 & 0.063 & c & c & c \\
\hline & $J$ & -0.062 & 0.022 & -0.103 & -0.016 & -0.108 & -0.046 & c & c & c \\
\hline & $\mathrm{L}$ & 0.035 & 0.026 & -0.015 & 0.086 & -0.011 & 0.051 & c & c & c \\
\hline & $M$ & -0.118 & 0.027 & -0.174 & -0.064 & -0.155 & -0.099 & c & c & c \\
\hline Season (early) & & 0.041 & 0.019 & 0.005 & 0.078 & 0.025 & 0.053 & 3.170 & 1 & 0.075 \\
\hline Year $(2017)^{f}$ & & -0.118 & 0.012 & -0.142 & -0.097 & -0.132 & -0.108 & 85.651 & 1 & $<0.001$ \\
\hline Sex $(\text { males })^{g}$ & & 0.018 & 0.013 & -0.007 & 0.042 & 0.010 & 0.024 & 1.840 & 1 & 0.175 \\
\hline Fruit intake & & -0.004 & 0.012 & -0.029 & 0.022 & -0.012 & 0.005 & 0.109 & 1 & 0.741 \\
\hline Leave intake & & 0.015 & 0.011 & -0.007 & 0.036 & 0.008 & 0.022 & 1.415 & 1 & 0.234 \\
\hline 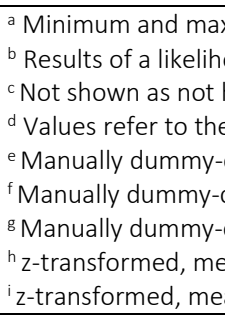 & $\begin{array}{l}\text { kimun } \\
\text { ood ro } \\
\text { having } \\
\text { over } \\
\text { coded } \\
\text { coded } \\
\text { coded } \\
\text { an an } \\
\text { an an }\end{array}$ & $\begin{array}{l}\text { of model es } \\
\text { o test com } \\
\text { meaningf } \\
\text { I test of the } \\
\text { vith group } \\
\text { ith the yea } \\
\text { vith "femal } \\
\text { SD of the o } \\
\text { SD of the o }\end{array}$ & $\begin{array}{l}\text { mates obt } \\
\text { aring the } f \\
\text { interpreta } \\
\text { ffect of th } \\
\text { " and seas } \\
\text { "2016" be } \\
\text { " being th } \\
\text { ginal value } \\
\text { sinal value }\end{array}$ & $\begin{array}{l}\text { ined when dr } \\
\text { I model with } \\
\text { on. See footr } \\
\text { predictor ("C } \\
\text { "late dry" b } \\
\text { g the referer } \\
\text { reference ca } \\
\text { were } 0.141 \text { a } \\
\text { were } 0.516 \text { a }\end{array}$ & $\begin{array}{l}\text { ping levels of } \\
\text { reduced mod } \\
\text { tes of Table S } \\
\text { bup") not the } \\
\text { ing the refere } \\
\text { e category } \\
\text { gory } \\
0.175 \text {, respe } \\
0.214 \text {, respe }\end{array}$ & $\begin{array}{l}\text { ndom effe } \\
\text { acking the } \\
\text { or details. } \\
\text { ecific level } \\
\text { e categorie }\end{array}$ & $\begin{array}{l}\text { sone at a } \\
\text { spective te } \\
\text { dicated in }\end{array}$ & e respective & & \\
\hline
\end{tabular}


Table S7 Results of post-hoc multiple comparisons of means using Tukey contrasts with adjusted p-values (Bonferroni) for comparisons of Phylogenetic diversities and Shannon indices among groups. Significant p-values are highlighted in bold.

\begin{tabular}{|c|c|c|c|c|}
\hline \multirow{2}{*}{ Groups } & \multicolumn{2}{|c|}{ PD } & \multicolumn{2}{|c|}{ Shannon index* } \\
\hline & Z & $p$ adj & Z & $p$ adj \\
\hline$F-E$ & 2.148 & 0.667 & -2.622 & 0.184 \\
\hline$F 1-E$ & 0.000 & 1.000 & 0.432 & 1.000 \\
\hline $\mathrm{F} 1-\mathrm{F}$ & -1.821 & 1.000 & 2.548 & 0.227 \\
\hline$G-E$ & 1.485 & 1.000 & 0.691 & 1.000 \\
\hline$G-F$ & -0.452 & 1.000 & 3.244 & 0.025 \\
\hline $\mathrm{G}-\mathrm{F} 1$ & 1.278 & 1.000 & 0.152 & 1.000 \\
\hline$J-E$ & -2.794 & 0.109 & -1.371 & 1.000 \\
\hline$J-F$ & -5.421 & $<0.001$ & 1.215 & 1.000 \\
\hline$J-F 1$ & -2.365 & 0.379 & -1.552 & 1.000 \\
\hline$J-G$ & -4.232 & $<0.001$ & -2.026 & 0.898 \\
\hline$L-E$ & 1.376 & 1.000 & 1.084 & 1.000 \\
\hline$L-F$ & -0.384 & 1.000 & 3.637 & 0.006 \\
\hline$L-F 1$ & 1.217 & 1.000 & 0.491 & 1.000 \\
\hline$L-G$ & 0.023 & 1.000 & 0.392 & 1.000 \\
\hline$L-J$ & 3.871 & 0.002 & 2.397 & 0.347 \\
\hline$M-E$ & -4.341 & $<0.001$ & -4.616 & $<0.001$ \\
\hline$M-F$ & -6.560 & $<0.001$ & -2.695 & 0.148 \\
\hline$M-F 1$ & -3.964 & 0.002 & -4.316 & $<0.001$ \\
\hline$M-G$ & -5.611 & $<0.001$ & -5.114 & $<0.001$ \\
\hline$M-J$ & -2.135 & 0.689 & -3.541 & 0.008 \\
\hline$M-L$ & -5.179 & $<0.001$ & -5.335 & $<0.001$ \\
\hline
\end{tabular}

\section{Effects of group size (PD)}

Table S8 Influence of group size on PDs (In-transformed) in seven groups of Verreaux's sifakas; results of the full model $\left(\right.$ GLMM; Nobservations $\left.=519, N_{I D}=41, N_{\text {Group }}=7\right)$.

\begin{tabular}{lccccccccc}
\hline \multicolumn{1}{c}{ Term } & Est & SE & Lower Cl & Upper Cl & Min $^{\mathrm{a}}$ & Max $^{\mathrm{a}}$ & $\chi^{2 \mathrm{~b}}$ & $d f$ & $P$ \\
\hline Intercept) & 4.505 & 0.025 & 4.452 & 4.556 & 4.496 & 4.534 & $\mathrm{c}$ & $\mathrm{c}$ & $\mathrm{c}$ \\
Group size $^{\mathrm{d}}$ & 0.015 & 0.028 & -0.043 & 0.084 & 0.000 & 0.057 & 0.298 & 1 & 0.585 \\
Year (2017) $^{\mathrm{e}}$ & -0.126 & 0.014 & -0.153 & -0.096 & -0.134 & -0.116 & 17.393 & 1 & $<0.001$ \\
Sex (male) & 0.014 & 0.016 & -0.018 & 0.045 & 0.004 & 0.022 & 0.776 & 1 & 0.378 \\
Season (early dry) $^{\mathrm{e}}$ & 0.052 & 0.018 & 0.015 & 0.089 & 0.035 & 0.080 & 5.251 & 1 & 0.022
\end{tabular}

\footnotetext{
a Minimum and maximum of model estimates obtained when dropping levels of random effects one at a time

${ }^{\mathrm{b}}$ Results of a likelihood ratio test comparing the full model with a reduced model lacking the respective term

${ }^{\mathrm{c}}$ Not shown as not having a meaningful interpretation. See footnotes of Table S1 for details.

${ }^{d}$ z-transformed, mean and SD of the original values were 6.387 and 2.082 , respectively

e Manually dummy-coded with the year "2016", "females" and "late dry season" being the reference categories
} 


\section{Effects of body contact within groups (PD)}

Table S9 Influence of body contact on PDs (In-transformed) in seven groups of Verreaux's sifakas; results of the full model $\left(\right.$ GLMM; $\left.N_{\text {Observations }}=427, N_{I D}=41, N_{\text {Group }}=7\right)$.

\begin{tabular}{|c|c|c|c|c|c|c|c|c|c|}
\hline Term & Est & SE & Lower $\mathrm{Cl}$ & Upper Cl & $\operatorname{Min}^{a}$ & $\operatorname{Max}^{\mathrm{a}}$ & $\chi^{2 b}$ & $d f$ & $P$ \\
\hline (Intercept) & 4.513 & 0.025 & 4.467 & 4.562 & 4.502 & 4.534 & c & c & c \\
\hline Body contact ${ }^{d}$ & -0.010 & 0.007 & -0.025 & 0.005 & -0.014 & -0.006 & 1.702 & 1 & 0.192 \\
\hline Year $(2017)^{\mathrm{e}}$ & -0.115 & 0.016 & -0.149 & -0.082 & -0.125 & -0.100 & 15.494 & 1 & $<0.001$ \\
\hline Sex $(\text { male })^{e}$ & 0.010 & 0.017 & -0.023 & 0.045 & -0.001 & 0.017 & 0.327 & 1 & 0.568 \\
\hline Season (early dry) & 0.038 & 0.018 & 0.001 & 0.073 & 0.018 & 0.047 & 3.299 & 1 & 0.069 \\
\hline \multicolumn{10}{|c|}{$\begin{array}{l}\text { a Minimum and maximum of model estimates obtained when dropping levels of random effects one at a time } \\
\text { b Results of a likelihood ratio test comparing the full model with a reduced model lacking the respective term } \\
\text { "Not shown as not having a meaningful interpretation. See footnotes of Table S1 for details. } \\
\text { d z-transformed, mean and SD of the original values were } 3.856 \text { and } 2.881 \text { in min/h, respectively } \\
\text { e Manually dummy-coded with the year "2016", "females" and "late dry season" being the reference categories }\end{array}$} \\
\hline
\end{tabular}




\section{Monthly changes of core phyla abundances}

Table S10 Kruskal-Wallis tests and Dunn's tests testing the difference in monthly abundance of 8 bacterial phyla in the gut microbiota of wild Verreaux's sifakas in 2016. Significant p-values $(<.05)$ are printed in bold

\begin{tabular}{|c|c|c|c|c|c|c|c|c|}
\hline \multirow{2}{*}{$\begin{array}{l}2016 \\
\text { Phylum }\end{array}$} & \multicolumn{3}{|c|}{ Kruskal-Wallis test } & \multicolumn{4}{|c|}{ Dunn's tests $p$-values } & \multirow{2}{*}{$\begin{array}{c}\text { Mean monthly relative } \\
\text { abundance (\%) }\end{array}$} \\
\hline & $\chi^{2}$ & $d f$ & $P$ & & April & May & September & \\
\hline \multirow[t]{4}{*}{ Actinobacteria } & 15.597 & 3 & 0.001 & April & - & - & - & 1.93 \\
\hline & & & & May & 0.308 & - & - & 2.21 \\
\hline & & & & September & 0.013 & 1 & - & 2.44 \\
\hline & & & & October & 1 & 0.152 & 0.004 & 1.87 \\
\hline \multirow[t]{4}{*}{ Bacteroidetes } & 0.553 & 3 & 0.907 & April & - & - & - & 22.76 \\
\hline & & & & May & - & - & - & 22.76 \\
\hline & & & & September & - & - & - & 22.90 \\
\hline & & & & October & - & - & - & 22.15 \\
\hline \multirow[t]{4}{*}{ Cyanobacteria } & 5.240 & 3 & 0.155 & April & - & - & - & 2.72 \\
\hline & & & & May & - & - & - & 2.42 \\
\hline & & & & September & - & - & - & 2.92 \\
\hline & & & & October & - & - & - & 2.77 \\
\hline \multirow[t]{4}{*}{ Fibrobacteres } & 24.397 & 3 & $<0.001$ & April & - & - & - & 3.96 \\
\hline & & & & May & 0.032 & - & - & 2.83 \\
\hline & & & & September & 0.284 & 1 & - & 3.18 \\
\hline & & & & October & 0.802 & $<0.001$ & 0.002 & 4.74 \\
\hline \multirow[t]{4}{*}{ Firmicutes } & 19.659 & 3 & $<0.001$ & April & - & - & - & 23.63 \\
\hline & & & & May & 0.005 & - & - & 27.06 \\
\hline & & & & September & $<0.001$ & 1 & - & 27.62 \\
\hline & & & & October & 0.531 & 0.557 & 0.083 & 25.42 \\
\hline \multirow[t]{4}{*}{ Proteobacteria } & 12.653 & 3 & $<0.001$ & April & - & - & - & 3.28 \\
\hline & & & & May & 1 & - & - & 3.18 \\
\hline & & & & September & 0.710 & 0.404 & - & 2.76 \\
\hline & & & & October & 0.030 & 0.010 & 1 & 2.42 \\
\hline \multirow[t]{4}{*}{ Synergistetes } & 9.247 & 3 & 0.026 & April & - & - & - & 0.71 \\
\hline & & & & May & 1 & - & - & 0.69 \\
\hline & & & & September & 1 & 1 & - & 0.73 \\
\hline & & & & October & 0.087 & 0.199 & 0.023 & 0.62 \\
\hline \multirow[t]{4}{*}{ Verrucomicrobia } & 45.856 & 3 & $<0.001$ & April & - & - & - & 4.27 \\
\hline & & & & May & 0.164 & - & - & 3.16 \\
\hline & & & & September & 1 & 0.021 & - & 4.54 \\
\hline & & & & October & $<0.001$ & $<0.001$ & 0.001 & 6.34 \\
\hline
\end{tabular}


Table S11 Kruskal-Wallis tests and Dunn's tests testing the difference in monthly abundance of 8 bacterial phyla in the gut microbiota of wild Verreaux's sifakas in 2017. Significant p-values (<.05) are printed in bold

\begin{tabular}{|c|c|c|c|c|c|c|c|c|}
\hline \multirow{2}{*}{$\begin{array}{l}2017 \\
\text { Phylum }\end{array}$} & \multicolumn{3}{|c|}{ Kruskal-Wallis test } & \multicolumn{4}{|c|}{ Dunn's tests $p$-values } & \multirow{2}{*}{$\begin{array}{c}\text { Mean monthly relative } \\
\text { abundance (\%) }\end{array}$} \\
\hline & $\chi^{2}$ & $d f$ & $P$ & & April & May & September & \\
\hline \multirow[t]{4}{*}{ Actinobacteria } & 30.488 & 3 & $<0.001$ & April & - & - & - & 2.32 \\
\hline & & & & May & 1 & - & - & 2.46 \\
\hline & & & & September & 0.002 & $<0.001$ & - & 1.85 \\
\hline & & & & October & 0.007 & $<0.001$ & 1 & 1.97 \\
\hline \multirow[t]{4}{*}{ Bacteroidetes } & 3.366 & 3 & 0.339 & April & - & - & - & 24.77 \\
\hline & & & & May & - & - & - & 23.24 \\
\hline & & & & September & - & - & - & 25.14 \\
\hline & & & & October & - & - & - & 24.06 \\
\hline \multirow[t]{4}{*}{ Cyanobacteria } & 6.133 & 3 & 0.105 & April & - & - & - & 2.92 \\
\hline & & & & May & - & - & - & 3.05 \\
\hline & & & & September & - & - & - & 3.18 \\
\hline & & & & October & - & - & - & 2.58 \\
\hline \multirow[t]{4}{*}{ Fibrobacteres } & 39.410 & 3 & $<0.001$ & April & - & - & - & 2.97 \\
\hline & & & & May & 0.199 & - & - & 2.32 \\
\hline & & & & September & 1 & 0.007 & - & 3.52 \\
\hline & & & & October & $<0.001$ & $<0.001$ & 0.037 & 4.72 \\
\hline \multirow[t]{4}{*}{ Firmicutes } & 25.132 & 3 & $<0.001$ & April & - & - & - & 24.59 \\
\hline & & & & May & $<0.001$ & - & - & 28.49 \\
\hline & & & & September & 0.001 & 1 & - & 28.18 \\
\hline & & & & October & $<0.001$ & 1 & 1 & 28.64 \\
\hline \multirow[t]{4}{*}{ Proteobacteria } & 51.328 & 3 & $<0.001$ & April & - & - & - & 3.14 \\
\hline & & & & May & 1 & - & - & 3.12 \\
\hline & & & & September & $<0.001$ & $<0.001$ & - & 2.18 \\
\hline & & & & October & $<0.001$ & $<0.001$ & 1 & 2.02 \\
\hline \multirow[t]{4}{*}{ Synergistetes } & 19.500 & 3 & $<0.001$ & April & - & - & - & 0.87 \\
\hline & & & & May & 0.235 & - & - & 0.79 \\
\hline & & & & September & 0.027 & 1 & - & 0.76 \\
\hline & & & & October & $<0.001$ & 0.095 & 0.808 & 0.67 \\
\hline \multirow[t]{4}{*}{ Verrucomicrobia } & 33.399 & 3 & $<0.001$ & April & - & - & - & 3.16 \\
\hline & & & & May & 1 & - & - & 3.05 \\
\hline & & & & September & $<0.001$ & $<0.001$ & - & 4.39 \\
\hline & & & & October & 0.004 & 0.001 & 1 & 4.03 \\
\hline
\end{tabular}




\section{(3) Further Analyses}

\section{a. Beta diversity (Bray-Curtis dissimilarity)}

\section{Effects of group membership, seasonality and individuals}

Samples from individuals living in the same group had lower dissimilarity scores than samples from individuals living in different groups (Table S12, Figure S3). Within groups, samples from the same individuals were more similar than samples between group members (GLMM: $\chi^{2}=23.609, \mathrm{df}=1, \mathrm{p}<$ $\left.0.001, R_{m}^{2}=0.12 / 0.91\right)$ (Table S13, Figure S4).

Within- and between-individual differences on microbiome compositions (Bray-Curtis)

Table S12 Results of Mantel tests comparing Bray-Curtis dissimilarities between seven groups of Verreaux's sifakas

\begin{tabular}{lccccc}
\hline Season & $\mathrm{n}_{\text {samples }}$ & nindividuals & $\bar{x}_{\text {same group }}$ & $\bar{x}_{\text {different group }}$ & $P$ \\
\hline early dry 2016 & 92 & 29 & 0.415 & 0.615 & $<0.001$ \\
late dry 2016 & 116 & 29 & 0.402 & 0.619 & $<0.001$ \\
early dry 2017 & 155 & 39 & 0.476 & 0.595 & $<0.001$ \\
late dry 2017 & 156 & 36 & 0.365 & 0.608 & $<0.001$ \\
\hline
\end{tabular}

Table S13 Comparing Bray-Curtis dissimilarities within and between group members in seven groups of Verreaux's sifakas; results of the full model (GLMM; Nobservations $\left.=455, N_{\text {ID dyads }}=153\right)$.

\begin{tabular}{|c|c|c|c|c|c|c|c|c|c|}
\hline Term & Est & SE & Lower $\mathrm{Cl}$ & Upper Cl & $\operatorname{Min}^{a}$ & $\operatorname{Max}^{a}$ & $\chi^{2 b}$ & $d f$ & $P$ \\
\hline (Intercept) & 0.426 & 0.022 & 0.384 & 0.469 & 0.407 & 0.454 & c & $\mathrm{c}$ & c \\
\hline Same ID (yes) ${ }^{d}$ & -0.106 & 0.021 & -0.147 & -0.064 & -0.126 & -0.091 & 23.609 & 1 & $<0.001$ \\
\hline Season ${ }^{\mathrm{e}}$ & & & & & & & $5.248^{f}$ & $3^{f}$ & $0.152^{f}$ \\
\hline late dry 2017 & -0.018 & 0.008 & -0.033 & -0.002 & -0.023 & -0.011 & c & c & c \\
\hline early dry 2016 & -0.009 & 0.008 & -0.025 & 0.008 & -0.014 & -0.005 & c & c & c \\
\hline early dry 2017 & -0.011 & 0.006 & -0.023 & 0.001 & -0.015 & -0.006 & c & c & c \\
\hline
\end{tabular}

\footnotetext{
a Minimum and maximum of model estimates obtained when dropping levels of random effects one at a time

${ }^{\mathrm{b}}$ Results of a likelihood ratio test comparing the full model with a reduced model lacking the respective term

c Not shown as not having a meaningful interpretation. See footnotes of Table A.1 for details

"Manually dummy-coded with the season "no" being the reference category

e Manually dummy-coded with the season "late dry 2016" being the reference category

${ }^{f}$ Values refer to the overall test of the effect of the predictor ("Season"), not the specific level indicated in the respective row
} 

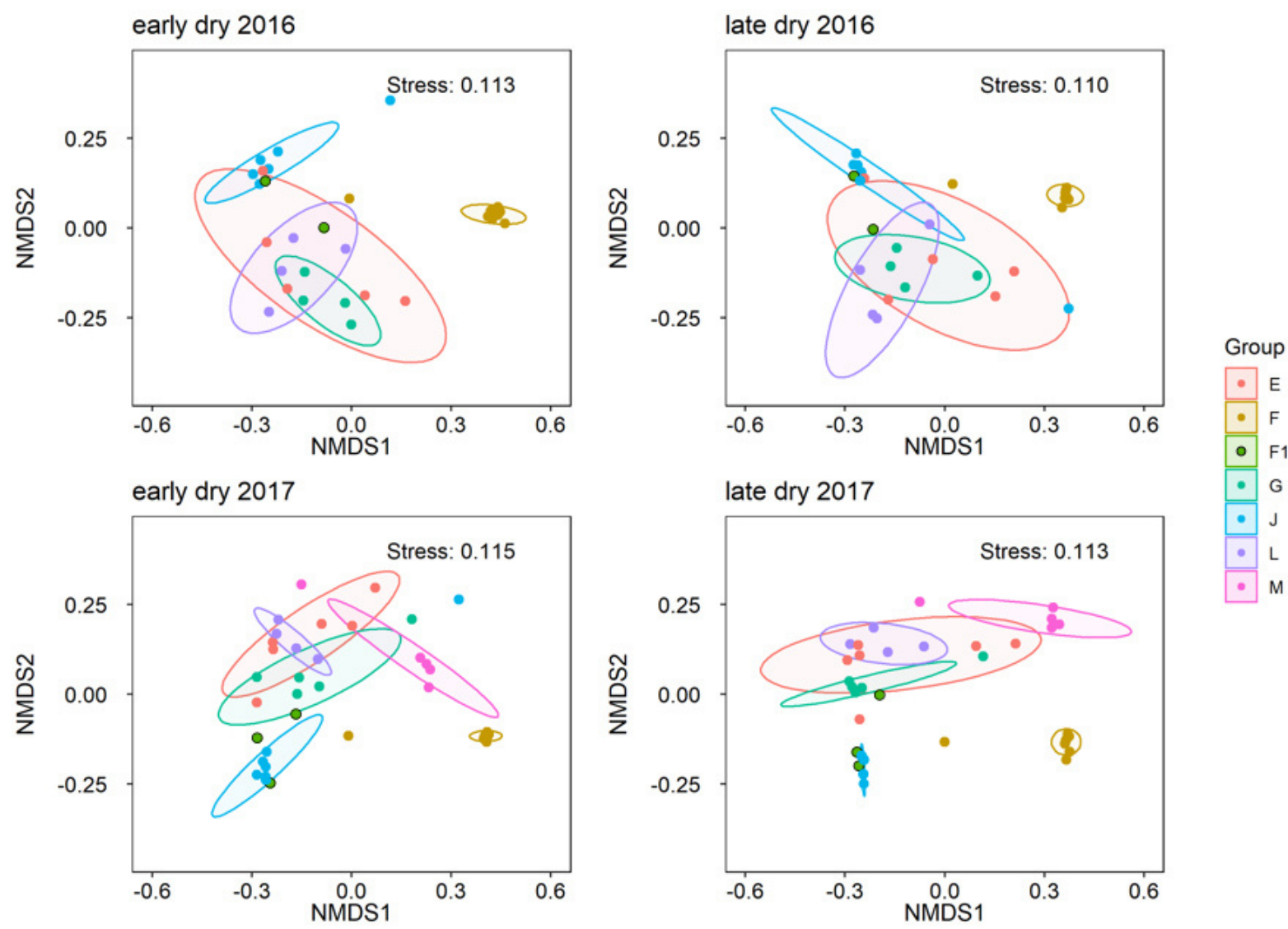

Figure S3 Nonmetric multidimensional scaling (NMDS) ordination of Verreaux's sifakas' gut bacterial composition data (Bray-Curtis) for each of the four field seasons. Data points represent individuals and colours indicate group membership. Ellipses indicate the $80 \%$ confidence ellipse for each group. For group F1, not enough data points were present to create ellipses, so we outlined the data points for better visibility. We collected several samples per individual per season, however, here we only plotted one data point per animal per season, based on average dyadic beta dissimilarity per field season, to facilitate the overview.

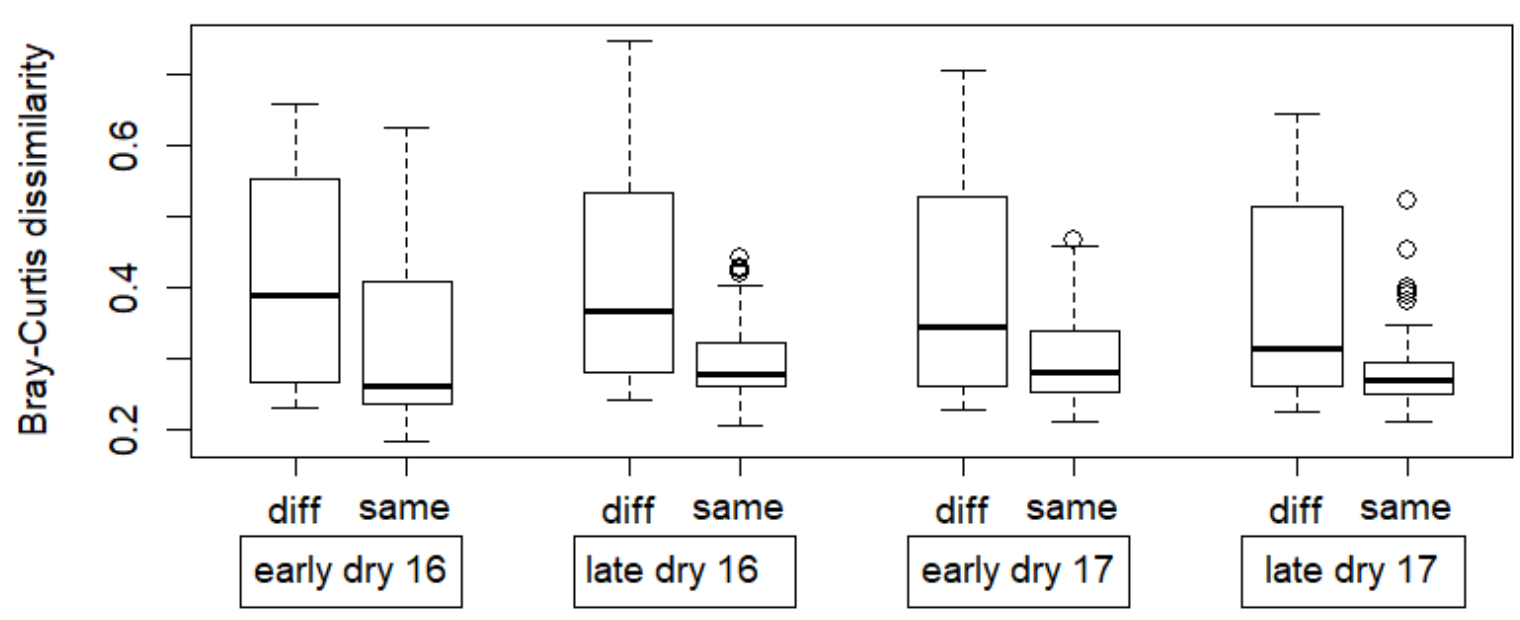

Figure S4 Comparison of Bray-Curtis dissimilarities between samples collected from the same individual and samples collected from different individuals living in the same group during for different field seasons. Boxplots comprise sample comparisons between all samples collected during the respective field seasons. Lines indicate median, upper and lower quartiles. Whiskers indicate $+/-1.5$ interquartile ranges and small circles beyond whiskers indicate outliers 


\section{Effects of body contact within groups (Bray-Curtis)}

The model examining effects of the time group members spent in body contact on Bray-Curtis dissimilarities was not significant $\left(\chi^{2}=0.831, \mathrm{df}=1, \mathrm{p}=0.362, \mathrm{R}_{\mathrm{m} / \mathrm{c}}^{2}=0.66 / 0.77\right)$ (Table S14).

Table S14 Influence of body contact on Bray-Curtis dissimilarities (sqrt-transformed) among group members of seven groups of Verreaux's sifakas; results of the full model (GLMM; Nobservations $\left.=2856, N_{\text {ID dyads }}=86\right)$.

\begin{tabular}{|c|c|c|c|c|c|c|c|c|c|}
\hline Term & Est & SE & Lower $\mathrm{Cl}$ & Upper Cl & $\operatorname{Min}^{a}$ & $\operatorname{Max}^{a}$ & $\chi^{2 b}$ & $d f$ & $P$ \\
\hline (Intercept) & -0.609 & 0.039 & -0.685 & -0.533 & -0.650 & -0.558 & c & c & c \\
\hline Body contact ${ }^{d}$ & 0.008 & 0.009 & -0.009 & 0.026 & -0.003 & 0.015 & 0.831 & 1 & 0.362 \\
\hline Related (yes) & -0.635 & 0.025 & -0.686 & -0.586 & -0.665 & -0.522 & 174.878 & 1 & $<0.001$ \\
\hline Season $^{f}$ & & & & & & & $6.385^{g}$ & $2^{g}$ & $0.041^{\mathrm{g}}$ \\
\hline late dry 2017 & -0.051 & 0.017 & -0.087 & -0.016 & -0.065 & -0.036 & c & c & c \\
\hline early dry 2017 & -0.034 & 0.017 & -0.067 & 0.003 & -0.062 & -0.020 & c & c & c \\
\hline \multicolumn{10}{|c|}{$\begin{array}{l}\text { "Minimum and maximum of model estimates obtained when dropping levels of random effects one at a time } \\
\text { b Results of a likelihood ratio test comparing the full model with a reduced model lacking the respective term } \\
\text { "Not shown as not having a meaningful interpretation. See footnotes of Table A.1 for details. } \\
\text { d z-transformed, mean and SD of the original values were } 0.771 \text { and } 1.187(\mathrm{~min} / \mathrm{h}) \text {, respectively } \\
\text { e Manually dummy-coded with the season "no" being the reference category } \\
\text { "Manually dummy-coded with the season "late dry } 2016 \text { " being the reference category }\end{array}$} \\
\hline
\end{tabular}

\section{Effects of maternal relatedness (Bray-Curtis)}

The interaction between maternal relatedness and group membership was not significant (Full-reduced model comparison: $\chi^{2}=1.056, d f=1, p=0.304$ ), so we excluded it from the model. The model examining the effects of maternal relatedness on Bray-Curtis dissimilarities between individuals was highly significant $\left(\chi^{2}=168.123, \mathrm{df}=1, \mathrm{p}<0.001, \mathrm{R}^{2} \mathrm{~m} / \mathrm{c}=0.62 / 0.96\right)$ (Table S15), as Bray-Curtis dissimilarities were lower among maternal relatives.

Table S15 Influence of maternal relatedness on Bray-Curtis dissimilarities in seven groups of Verreaux's sifakas; results of the full model (GLMM; Nobservations = 1439, NID dyads $=502)$.

\begin{tabular}{|c|c|c|c|c|c|c|c|c|c|}
\hline Term & Est & SE & Lower $\mathrm{Cl}$ & Upper Cl & $\operatorname{Min}^{a}$ & $\operatorname{Max}^{a}$ & $\chi^{2 b}$ & $d f$ & $P$ \\
\hline (Intercept) & 0.625 & 0.004 & 0.616 & 0.633 & -0.237 & -0.230 & c & c & c \\
\hline Related (yes) ${ }^{\mathrm{d}}$ & -0.233 & 0.016 & -0.264 & -0.200 & -0.103 & -0.096 & 168.129 & 1 & $<0.001$ \\
\hline Same group (yes) & -0.100 & 0.015 & -0.129 & -0.072 & -0.237 & -0.230 & 45.040 & 1 & $<0.001$ \\
\hline Season $^{f}$ & & & & & & & $31.516^{g}$ & $3^{g}$ & $<0.001^{\mathrm{g}}$ \\
\hline late dry 2017 & -0.014 & 0.003 & -0.020 & -0.009 & -0.015 & -0.014 & c & c & c \\
\hline early dry 2016 & -0.007 & 0.003 & -0.012 & -0.002 & -0.007 & -0.006 & c & c & c \\
\hline early dry 2017 & -0.008 & 0.002 & -0.013 & -0.003 & -0.009 & -0.008 & c & c & c \\
\hline \multicolumn{10}{|c|}{$\begin{array}{l}\text { "Minimum and maximum of model estimates obtained when dropping levels of random effects one at a time } \\
\text { "Results of a likelihood ratio test comparing the full model with a reduced model lacking the respective term } \\
\text { "Not shown as not having a meaningful interpretation. See footnotes of Table A.1 for details. } \\
\text { dManually dummy-coded with the season "no" being the reference category } \\
\text { e Manually dummy-coded with the season "no" being the reference category } \\
\text { fManually dummy-coded with the season "late dry 2016" being the reference category }\end{array}$} \\
\hline
\end{tabular}




\section{Effects of habitat dissimilarity, overlap and diet dissimilarity between groups (Bray-Curtis)}

Habitat and Bray-Curtis dissimilarities between groups were not correlated (Pearson: $r=-0.091, n_{\text {Group dyads }}$ $=21, p=0.694)$. The models examining effects of habitat overlap and diet dissimilarities on groups' BrayCurtis dissimilarities were not significant either (habitat overlap: $\chi^{2}=0.027$, $d f=1, p=0.870, R^{2} \mathrm{~m} / \mathrm{c}=$ 0.01/0.97; diet dissimilarity: $\left.\chi^{2}=0.210, \mathrm{df}=1, \mathrm{p}=0.647, \mathrm{R}_{\mathrm{m} / \mathrm{c}}^{2}=0.01 / 0.97\right)$ (Tables S16 \& S17).

Table S16 Influence of habitat overlap on Bray-Curtis dissimilarities (In-transformed) among seven groups of Verreaux's sifakas; results of the full model (GLMM; Nobservations $\left.=72, \mathrm{~N}_{\text {Group dyads }}=21\right)$.

\begin{tabular}{|c|c|c|c|c|c|c|c|c|c|}
\hline Term & Est & SE & Lower $\mathrm{Cl}$ & Upper Cl & $\operatorname{Min}^{a}$ & $\operatorname{Max}^{a}$ & $\chi^{2 b}$ & $d f$ & $P$ \\
\hline (Intercept) & -0.506 & 0.020 & -0.544 & -0.467 & -0.512 & -0.494 & c & c & c \\
\hline Overlap ${ }^{d}$ & 0.001 & 0.008 & -0.015 & 0.017 & -0.005 & 0.009 & 0.027 & 1 & 0.870 \\
\hline Season ${ }^{\mathrm{e}}$ & & & & & & & $7.863^{f}$ & $3^{f}$ & 0.049 \\
\hline late dry 2017 & -0.018 & 0.009 & -0.036 & -0.001 & -0.021 & -0.014 & c & c & c \\
\hline early dry 2016 & -0.029 & 0.012 & -0.053 & -0.007 & -0.036 & -0.019 & c & c & c \\
\hline early dry 2017 & -0.011 & 0.006 & -0.023 & 0.002 & -0.014 & -0.008 & c & c & c \\
\hline \multicolumn{10}{|c|}{$\begin{array}{l}\text { "Minimum and maximum of model estimates obtained when dropping levels of random effects one at a time } \\
\text { b Results of a likelihood ratio test comparing the full model with a reduced model lacking the respective term } \\
\text { "Not shown as not having a meaningful interpretation. See footnotes of Table A.1 for details } \\
\text { d z-transformed, mean and SD of the original values were } 0.045 \text { and } 0.063 \text {, respectively } \\
\text { e Manually dummy-coded with the season "late dry } 2016 \text { " being the reference category }\end{array}$} \\
\hline
\end{tabular}

Table S17 Influence of diet dissimilarity (Bray-Curtis) on Bray-Curtis dissimilarities (In-transformed) among seven groups of Verreaux's sifakas; results of the full model $\left(G L M M ; N_{\text {observations }}=57, N_{\text {Group dyads }}=21\right)$.

\begin{tabular}{|c|c|c|c|c|c|c|c|c|c|}
\hline Term & Est & SE & Lower $\mathrm{Cl}$ & Upper $\mathrm{Cl}$ & $\operatorname{Min}^{a}$ & $\operatorname{Max}^{a}$ & $\chi^{2 b}$ & $d f$ & $P$ \\
\hline (Intercept) & -0.506 & 0.020 & -0.543 & -0.468 & -0.513 & -0.494 & c & c & c \\
\hline Diet dissimiliarity ${ }^{d}$ & 0.002 & 0.004 & -0.006 & 0.010 & 0.000 & 0.003 & 0.210 & 1 & 0.647 \\
\hline Season $^{\mathrm{e}}$ & & & & & & & $4.676^{f}$ & $2^{f}$ & $0.097^{\dagger}$ \\
\hline late dry 2017 & -0.017 & 0.009 & -0.035 & 0.000 & -0.021 & -0.013 & c & c & c \\
\hline early dry 2017 & -0.011 & 0.006 & -0.024 & 0.001 & -0.016 & -0.008 & c & c & c \\
\hline
\end{tabular}

\footnotetext{
a Minimum and maximum of model estimates obtained when dropping levels of random effects one at a time

${ }^{b}$ Results of a likelihood ratio test comparing the full model with a reduced model lacking the respective term

c Not shown as not having a meaningful interpretation. See footnotes of Table A.1 for details.

${ }^{d}$ z-transformed, mean and SD of the original values were 0.592 and 0.132 , respectively

e Manually dummy-coded with the season "late dry 2016" being the reference category

${ }^{f}$ Values refer to the overall test of the effect of the predictor ("Season"), not the specific level indicated in the respective row
} 


\section{b. Alpha diversity (Shannon index)}

\section{Effects of group membership, season, study year and diet}

The model examining effects of group membership, season (early dry vs. late dry), study year and diet on individual Shannon indices was significant $\left(\chi^{2}=46.152, d f=9, p<0.001, R_{m / c}^{2}=0.35 / 0.51\right)$ (Table S18). In detail, group membership and study year affected individual alpha diversity. Members of group $M$ had lower Shannon indices than all groups except for group F. Group F had lower Shannon indices than groups E, G, J and L (Figure S5 a). In 2017, all animals had lower Shannon indices than in 2016 (Figure S5 b). No correlations were found for seasons and fruit and leave intake rates (Figure $\mathrm{S} 5 \mathrm{c}, \mathrm{d} \& \mathrm{e}$ ).

\section{Effects of group size (Shannon)}

The second model, examining effects of group size on Shannon indices was not significant $\left(\chi^{2}=3.407\right.$, $\mathrm{df}$ $=1, p=0.065, R_{m / c}^{2}=0.16 / 0.52$ ) (Table S19) (Figure S5 f).

\section{Effects of body contact within groups (Shannon)}

The model examining effects of time spent in body contact within groups was not significant $\left(\chi^{2}=0.108\right.$, $\mathrm{df}=1, \mathrm{p}=0.742, \mathrm{R}_{\mathrm{m} / \mathrm{c}}=0.11 / 0.54$ ) (Table S20, Figure S5 g).

Table S18 Influence of various factors on Shannon indeces (In-transformed) in seven groups of Verreaux's sifakas; results of the full model (GLMM; $N_{\text {Observations }}=519, N_{I D}=41, N_{\text {Group }}=7$ ).

\begin{tabular}{|c|c|c|c|c|c|c|c|c|c|c|}
\hline Term & & Est & SE & Lower $\mathrm{Cl}$ & Upper Cl & $\operatorname{Min}^{a}$ & $\operatorname{Max}^{a}$ & $\chi^{2 b}$ & $d f$ & $P$ \\
\hline (Intercept) & & 1.637 & 0.012 & 1.612 & 1.660 & 1.601 & 1.641 & c & c & c \\
\hline \multirow[t]{6}{*}{ Groupe } & $\mathrm{F}$ & -0.033 & 0.013 & -0.058 & -0.007 & -0.041 & -0.028 & $20.295^{d}$ & $6^{d}$ & $0.002^{d}$ \\
\hline & $\mathrm{F} 1$ & 0.007 & 0.017 & -0.025 & 0.041 & -0.006 & 0.040 & c & c & c \\
\hline & G & 0.010 & 0.015 & -0.018 & 0.04 & 0.004 & 0.044 & c & c & c \\
\hline & J & -0.019 & 0.014 & -0.045 & 0.009 & -0.025 & 0.015 & c & c & c \\
\hline & $\mathrm{L}$ & 0.016 & 0.015 & -0.014 & 0.048 & 0.010 & 0.050 & c & c & c \\
\hline & $M$ & -0.071 & 0.015 & -0.101 & -0.042 & -0.085 & -0.038 & c & c & c \\
\hline \multicolumn{2}{|c|}{ Season (early dry) } & -0.008 & 0.006 & -0.020 & 0.005 & -0.011 & -0.004 & 1.276 & 1 & 0.259 \\
\hline \multicolumn{2}{|l|}{ Year $(2017)^{f}$} & -0.036 & 0.004 & -0.044 & -0.029 & -0.038 & -0.034 & 17.664 & 1 & $<0.001$ \\
\hline \multicolumn{2}{|l|}{ Sex $(\text { males })^{g}$} & -0.008 & 0.008 & -0.025 & 0.007 & -0.014 & -0.003 & 1.039 & 1 & 0.308 \\
\hline \multicolumn{2}{|l|}{ Fruit intake ${ }^{h}$} & -0.005 & 0.003 & -0.011 & 0.002 & -0.008 & -0.002 & 1.564 & 1 & 0.211 \\
\hline \multicolumn{2}{|l|}{ Leave intake } & 0.000 & 0.003 & -0.006 & 0.006 & -0.001 & 0.001 & 0.000 & 1 & 0.992 \\
\hline 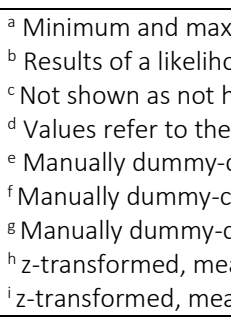 & $\begin{array}{l}\text { kimum } \\
\text { Dod ra } \\
\text { having } \\
\text { over } \\
\text { coded } \\
\text { oded } \\
\text { coded } \\
\text { an an } \\
\text { an anc }\end{array}$ & $\begin{array}{l}f \text { model es } \\
\text { o test comb } \\
\text { meaningf } \\
\text { test of the } \\
\text { vith group } \\
\text { ith the yea } \\
\text { ith "female } \\
\text { SD of the o } \\
\text { DD of the or }\end{array}$ & $\begin{array}{l}\text { mates obt } \\
\text { ring the } f \text {, } \\
\text { interpreta } \\
\text { ffect of th } \\
\text { " and seas } \\
\text { "2016" be } \\
\text { " being th } \\
\text { sinal value } \\
\text { inal value }\end{array}$ & $\begin{array}{l}\text { ined when dro } \\
\text { I model with a } \\
\text { ion. See footn } \\
\text { predictor ("G } \\
\text { on "late dry" b } \\
\text { g the referenc } \\
\text { reference cat } \\
\text { were } 0.141 \mathrm{an} \\
\text { were } 0.516 \text { ar }\end{array}$ & $\begin{array}{l}\text { ping levels of } \\
\text { reduced mode } \\
\text { tes of Table S1 } \\
\text { pup"), not the } \\
\text { ing the referer } \\
\text { e category } \\
\text { gory } \\
0.175 \text {, respe } \\
0.214 \text {, respe }\end{array}$ & $\begin{array}{l}\text { ndom effe } \\
\text { lacking the } \\
\text { or details. } \\
\text { pecific leve } \\
\text { e categorie }\end{array}$ & $\begin{array}{l}\text { s one at a } \\
\text { spective te } \\
\text { ndicated in }\end{array}$ & $\begin{array}{l}\text { ne } \\
\mathrm{m} \\
\text { he respective }\end{array}$ & row & \\
\hline
\end{tabular}


Table S19 Influence of group size on Shannon indices (In-transformed) in seven groups of Verreaux's sifakas; results of the full model (GLMM; Nobservations $\left.=519, N_{I D}=41, N_{\text {Group }}=7\right)$.

\begin{tabular}{|c|c|c|c|c|c|c|c|c|c|}
\hline Term & Est & SE & Lower $\mathrm{Cl}$ & Upper Cl & $\operatorname{Min}^{a}$ & $\operatorname{Max}^{a}$ & $\chi^{2 b}$ & $d f$ & $P$ \\
\hline (Intercept) & 1.622 & 0.011 & 1.600 & 1.645 & 1.616 & 1.634 & c & c & c \\
\hline Group size $^{d}$ & -0.012 & 0.006 & -0.026 & 0.001 & -0.019 & -0.008 & 3.407 & 1 & 0.065 \\
\hline Year $(2017)^{\mathrm{e}}$ & -0.033 & 0.004 & -0.041 & -0.026 & -0.035 & -0.030 & 14.554 & 1 & $<0.001$ \\
\hline Sex $(\text { male })^{e}$ & -0.006 & 0.009 & -0.023 & 0.013 & -0.012 & -0.001 & 0.386 & 1 & 0.534 \\
\hline Season (early dry) & -0.017 & 0.004 & -0.026 & -0.008 & -0.022 & -0.016 & 10.628 & 1 & 0.001 \\
\hline \multicolumn{10}{|c|}{$\begin{array}{l}\text { "Minimum and maximum of model estimates obtained when dropping levels of random effects one at a time } \\
\text { b Results of a likelihood ratio test comparing the full model with a reduced model lacking the respective term } \\
\text { " Not shown as not having a meaningful interpretation. See footnotes of Table S1 for details. } \\
\text { d z-transformed, mean and SD of the original values were } 6.387 \text { and } 2.082 \text {, respectively } \\
\text { e Manually dummy-coded with the year "2016", "females" and "late dry season" being the reference categories }\end{array}$} \\
\hline
\end{tabular}

Table S20 Influence of body contact on Shannon indices (In-transformed) in seven groups of Verreaux's sifakas; results of the full model (GLMM; Nobservations $\left.=427, N_{I D}=41, N_{\text {Group }}=7\right)$.

\begin{tabular}{|c|c|c|c|c|c|c|c|c|c|}
\hline Term & Est & SE & Lower $\mathrm{Cl}$ & Upper Cl & $\operatorname{Min}^{a}$ & $\operatorname{Max}^{a}$ & $\chi^{2 b}$ & $d f$ & $P$ \\
\hline (Intercept) & 1.617 & 0.012 & 1.600 & 1.645 & 1.610 & 1.629 & c & $\mathrm{c}$ & c \\
\hline Body contact ${ }^{d}$ & 0.001 & 0.002 & -0.026 & 0.001 & 0.000 & 0.002 & 0.108 & 1 & 0.742 \\
\hline Year $(2017)^{\mathrm{e}}$ & -0.025 & 0.005 & -0.041 & -0.026 & -0.029 & -0.021 & 7.886 & 1 & 0.005 \\
\hline Sex $(\text { male })^{\mathrm{e}}$ & -0.006 & 0.010 & -0.023 & 0.013 & -0.013 & -0.001 & 0.397 & 1 & 0.529 \\
\hline Season (early dry) & -0.021 & 0.005 & -0.026 & -0.008 & -0.024 & -0.018 & 11.167 & 1 & 0.001 \\
\hline \multicolumn{10}{|c|}{$\begin{array}{l}\text { "Minimum and maximum of model estimates obtained when dropping levels of random effects one at a time } \\
\text { b Results of a likelihood ratio test comparing the full model with a reduced model lacking the respective term } \\
\text { c Not shown as not having a meaningful interpretation. See footnotes of Table S1 for details. } \\
\text { d z-transformed, mean and SD of the original values were } 3.856 \text { and } 2.881 \text { in min/h, respectively } \\
\text { e Manually dummy-coded with the year "2016", "females" and "late dry season" being the reference categories }\end{array}$} \\
\hline
\end{tabular}



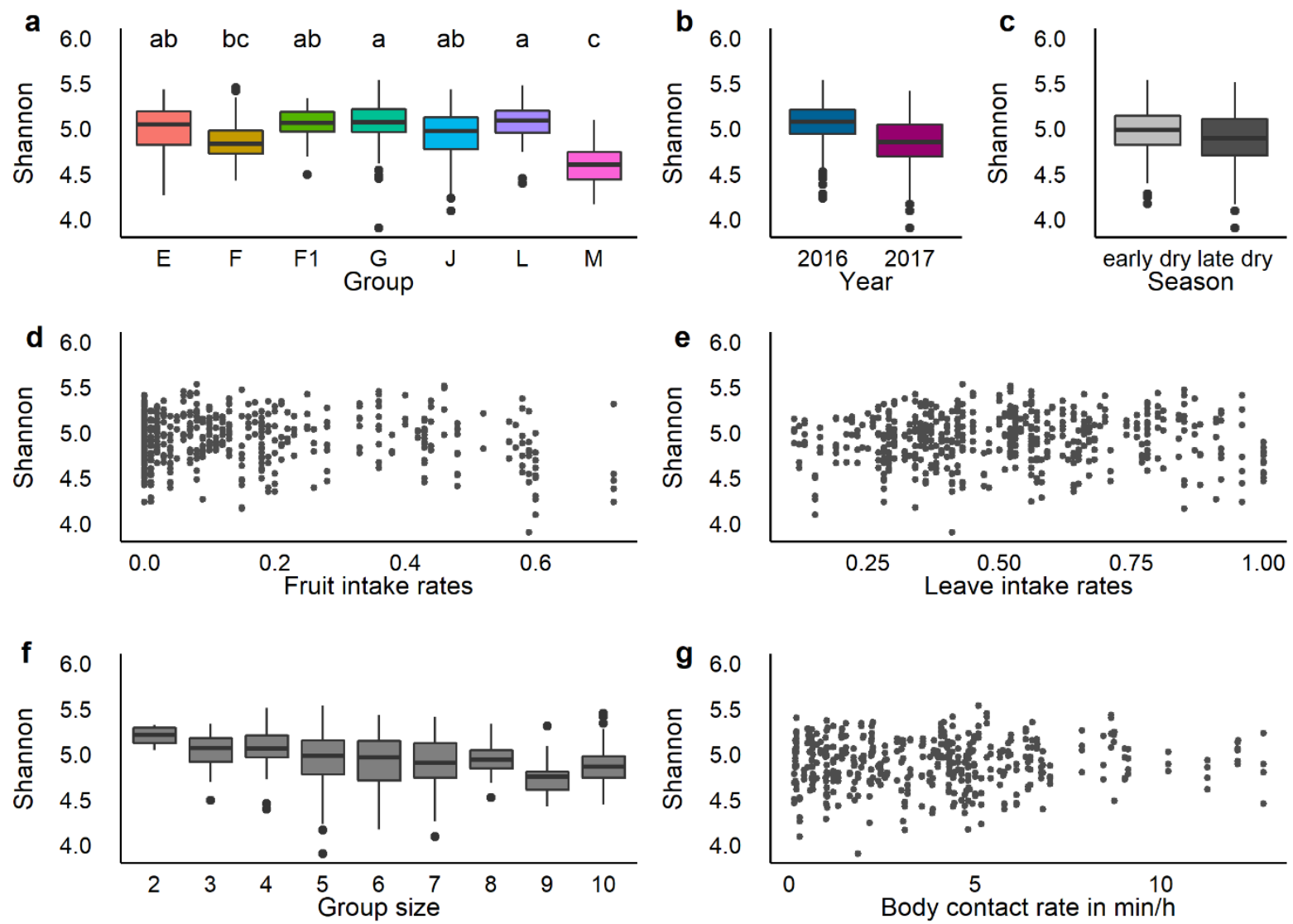

Figure S5 Influence of various factors on alpha diversity (Shannon index). Coloured graphs indicate significant effects. a, b $\&$ c) Influence of group identity, study year and season. Boxplots comprise all individual samples with lines indicating median, upper and lower quartiles. Whiskers indicate $+/-1.5$ interquartile ranges and small circles beyond whiskers indicate outliers. d \& e) Influence of mean fruit and leave intake rates per field season. f) Influence of mean group size per field season. g) Influence of mean time spent in body contact per field season 


\section{c. Comparisons with Perofsky et al. (2017): Social network statistics and microbiomes}

Like Perofsky et al. (2017), we constructed social networks based on grooming interactions for each of three field seasons from the data set described in the main paper. We calculated grooming indices as the time two individuals spent grooming divided by the combined focal observation times of both individual. Grooming indices were used as weights for network edges. We described the social connectivity within groups via edge densities, i.e. the number of edges divided by the number of possible edges. Moreover, we calculated inverse weighted path lengths (i.e. the smallest weight of inverse weights of edges) as a measure for social distance between each group member dyad and we computed weighted degree centrality for each individual (i.e. the sum of each individual's edge weights) from the grooming network. Social networks and network statistics were constructed with the package igraph (version 1.2.4.1) (Csárdi and Nepusz, 2006).

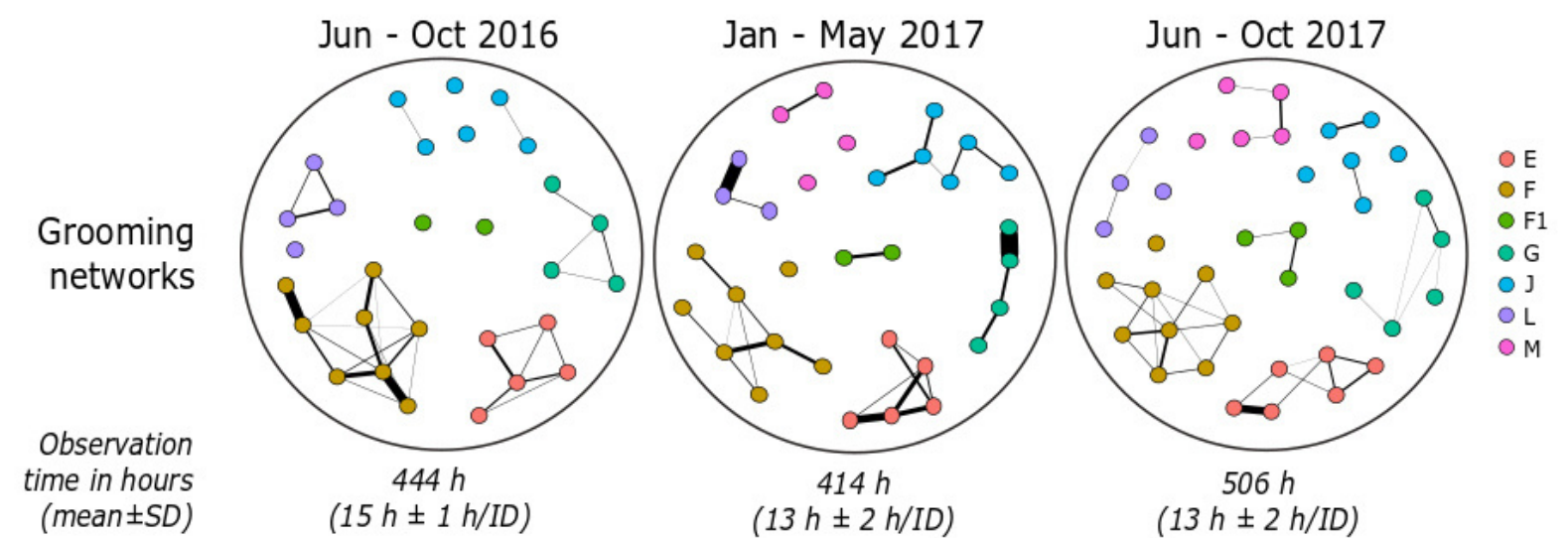

Figure S6 Social networks based on grooming interactions observed in the 3 months prior to and during field seasons of microbiome sampling among seven sifaka groups. Nodes represent individuals and lines represent grooming interactions between individuals with thicker lines reflecting higher grooming indices.

Based on the grooming networks, we conducted three analyses in similar manner as Perofsky et al. (2017): 1) To examine whether individuals in groups with higher average edge densities, i.e. social connectedness, share more similar microbiomes, we used Pearson correlations of average edge densities per group per field season with average dyadic beta diversities between group members. 2) We examined the effects of "social distance" (measured as inverse weighted path lengths) on GUniFrac distances between group members by applying GLMMs. Individual dyads and group ID were used as random effects and field seasons as random slopes. To control for effects of field season and maternal relatedness (see below), we included both factors as fixed effects. 3) To assess whether group members with stronger ties (measured as weighted degree centrality) have higher PDs, we applied another GLMM. We included study year and sex as fixed effects, group and animal ID as random effects and study year as random slope. Additionally, we included weighted degree centrality as random slope per ID. 


\section{Results and discussion of social network statistics}

1) Pearson correlations examining links between groups' edge densities and individuals' GUniFrac distances and Bray-Curtis dissimilarities yielded in varying results across the field seasons. There was no correlation during the late dry season 2016 (GUniFrac: $r=0.054, p=0.665$; Bray-Curtis: $r=0.007, p=$ 0.957), while during the early and late dry season 2017 we found groups with greater social connectedness to have less homogenous microbiomes (early dry 2017: GUniFrac: $r=0.377, p<0.001$; Bray-Curtis: $r=0.303, p=0.002$; late dry 2017: GUniFrac: $r=0.288, p=0.007$; Bray-Curtis: $r=0.345, p=$ 0.001) (Figure S7).

2) The models examining the effects of social distance on GUniFrac distances between group members was significant $\left(\chi^{2}=4.765, d f=1, p=0.029, R_{m}^{2} / c=0.54 / 0.62\right)$, while the model on Bray-Curtis dissimilarities was not $\left(\chi^{2}=1.825, \mathrm{df}=1, p=0.177, \mathrm{R}_{\mathrm{m} / \mathrm{c}}^{2}=0.66 / 0.79\right)$. Thus, dyads with stronger grooming relationships shared less closely related bacteria, however, this effect was fairly weak (Table S21).

\section{Late dry 2016}
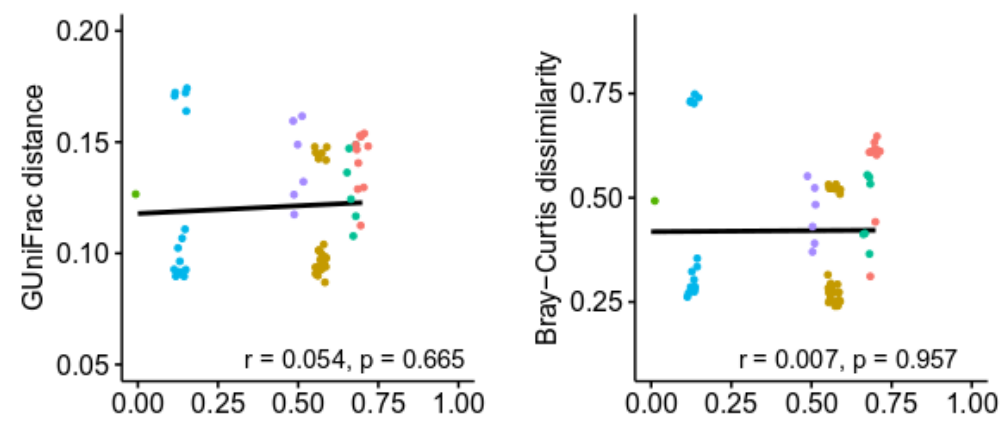

\section{Early dry 2017}
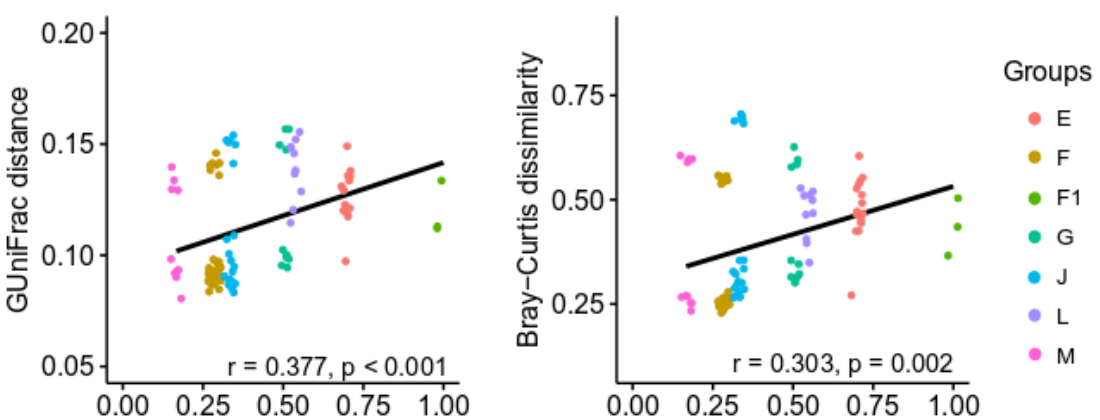

\section{Late dry 2017}
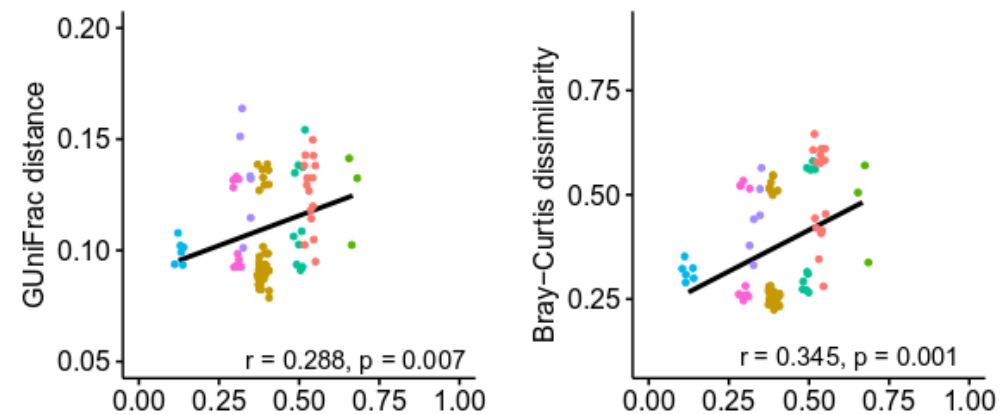

Within-group network edge density
Figure S7 Effects of groups' average edge densities on microbiome dissimilarities among group members. Data points represent mean beta diversity between group members per field seasons. Lines indicate regression lines of Pearson correlations. 
Table S21 Influence of social distance (measured as weighted path lengths) on GUniFrac distances (In-transformed) and BrayCurtis dissimilarities (sqrt-transformed) among group members of seven groups of Verreaux's sifakas; results of the full model $\left(\right.$ GLMM; Nobservations $\left.=2252, N_{\text {ID dyads }}=76, N_{\text {Grous }}=6\right)$.

\begin{tabular}{|c|c|c|c|c|c|c|c|c|c|}
\hline Term & Est & SE & Lower $\mathrm{Cl}$ & Upper Cl & $\operatorname{Min}^{a}$ & $\operatorname{Max}^{a}$ & $\chi^{2 b}$ & $d f$ & $P$ \\
\hline \multicolumn{10}{|c|}{ GUniFrac Model } \\
\hline (Intercept) & 0.377 & 0.003 & 0.370 & 0.384 & 0.374 & 0.381 & c & c & c \\
\hline Social distance ${ }^{d}$ & 0.003 & 0.001 & 0.000 & 0.006 & 0.001 & 0.005 & 4.765 & 1 & 0.029 \\
\hline Related (yes) & -0.062 & 0.003 & -0.068 & -0.057 & -0.067 & -0.052 & 126.925 & 1 & $<0.001$ \\
\hline Season $^{f}$ & & & & & & & $5.603^{g}$ & $2^{g}$ & $0.061^{\mathrm{g}}$ \\
\hline late dry 2017 & -0.009 & 0.006 & -0.022 & 0.003 & -0.015 & 0.000 & c & c & c \\
\hline early dry 2017 & -0.008 & 0.002 & -0.013 & -0.004 & -0.010 & -0.005 & c & c & c \\
\hline \multicolumn{10}{|c|}{ Bray-Curtis Model } \\
\hline (Intercept) & -0.601 & 0.053 & -0.702 & -0.492 & -0.647 & -0.539 & c & c & c \\
\hline Social distance ${ }^{d}$ & 0.015 & 0.011 & -0.007 & 0.038 & 0.000 & 0.044 & 1.825 & 1 & 0.177 \\
\hline Related (yes) & -0.649 & 0.022 & -0.693 & -0.608 & -0.675 & -0.530 & 166.756 & 1 & $<0.001$ \\
\hline Season $^{f}$ & & & & & & & $2.956^{g}$ & $2^{g}$ & $0.228^{g}$ \\
\hline late dry 2017 & -0.044 & 0.048 & -0.135 & 0.049 & -0.077 & -0.007 & c & c & c \\
\hline early dry 2017 & -0.038 & 0.019 & -0.083 & 0.000 & -0.052 & -0.021 & c & c & c \\
\hline $\begin{array}{l}\text { a Minimum and maximu } \\
\text { b Results of a likelihood } \\
\text { ' Not shown as not havi } \\
\text { d z-transformed, mean a } \\
\text { e Manually dummy-code } \\
{ }^{\mathrm{f}} \text { Manually dummy-code } \\
{ }^{\mathrm{g}} \text { Values refer to the ove }\end{array}$ & $\begin{array}{l}\text { of model es } \\
\text { io test com } \\
\text { a meaningf } \\
\text { SD of the c } \\
\text { with the sea } \\
\text { vith the sea } \\
\text { test of the }\end{array}$ & $\begin{array}{l}\text { mates obt } \\
\text { ring the fi } \\
\text { interpreta } \\
\text { ginal value } \\
\text { on "no" be } \\
\text { in "late dr } \\
\text { ffect of th }\end{array}$ & $\begin{array}{l}\text { ned when drc } \\
\text { I model with a } \\
\text { on. See footn } \\
\text { were } 29.951 \\
\text { g the referen } \\
2016 \text { " being } t \\
\text { predictor ("SE }\end{array}$ & $\begin{array}{l}\text { ping levels of } \\
\text { educed mode } \\
\text { es of Table A. } \\
\text { h } 32.371 \text {, res } \\
\text { category } \\
\text { e reference ca } \\
\text { son"), not the }\end{array}$ & $\begin{array}{l}\text { ndom effe } \\
\text { acking the } \\
\text { for details. } \\
\text { ectively } \\
\text { gory } \\
\text { pecific leve }\end{array}$ & $\begin{array}{l}\text { one at a ti } \\
\text { spective te }\end{array}$ & e & & \\
\hline
\end{tabular}

3) Individuals with stronger ties within their groups (measured as weighted degree centrality) did not have greater PDs $\left(\chi^{2}=0.710, d f=1, p=0.399, R^{2} / c=0.12 / 0.26\right)$ or Shannon indices $\left(\chi^{2}=0.125, d f=\right.$ $\left.1, p=0.723, R_{m / c}^{2}=0.11 / 0.56\right)($ Table S22). 
Table S22 Influence of individual weighted degree centrality on GUniFrac distances (In-transformed) and Bray-Curtis dissimilarities (sqrt-transformed) among group members of seven groups of Verreaux's sifakas; results of the full model $\left(\right.$ GLMM; Nobservations $\left.=396, \mathrm{~N}_{\text {Groups }}=7, \mathrm{~N}_{\mathrm{ID}}=41\right)$.

\begin{tabular}{|c|c|c|c|c|c|c|c|c|c|}
\hline Term & Est & SE & Lower $\mathrm{Cl}$ & Upper Cl & $\operatorname{Min}^{a}$ & $\mathrm{Max}^{\mathrm{a}}$ & $\chi^{2 b}$ & $d f$ & $P$ \\
\hline \multicolumn{10}{|c|}{ PD Model } \\
\hline (Intercept) & 4.523 & 0.023 & 4.480 & 4.567 & 4.512 & 4.579 & c & c & c \\
\hline$W D C^{d}$ & 0.008 & 0.010 & -0.012 & 0.029 & 0.001 & 0.021 & 0.710 & 1 & 0.399 \\
\hline Year $(2017)^{\mathrm{e}}$ & -0.117 & 0.016 & -0.147 & -0.084 & -0.165 & -0.107 & 16.361 & 1 & $<0.001$ \\
\hline $\operatorname{Sex}(m)^{e}$ & 0.002 & 0.016 & -0.03 & 0.031 & -0.007 & 0.007 & 0.012 & 1 & 0.914 \\
\hline Season (early dry) & 0.033 & 0.018 & -0.002 & 0.067 & 0.007 & 0.043 & 3.007 & 1 & 0.083 \\
\hline \multicolumn{10}{|c|}{ Shannon Model } \\
\hline (Intercept) & 1.618 & 0.013 & 1.593 & 1.644 & 1.610 & 1.629 & c & c & c \\
\hline$W C^{d}$ & -0.001 & 0.003 & -0.008 & 0.006 & -0.003 & 0.001 & 0.125 & 1 & 0.723 \\
\hline Year $(2017)^{\mathrm{e}}$ & -0.023 & 0.006 & -0.035 & -0.012 & -0.028 & -0.020 & 7.150 & 1 & 0.007 \\
\hline $\operatorname{Sex}(m)^{e}$ & -0.008 & 0.010 & -0.027 & 0.013 & -0.015 & -0.002 & 0.564 & 1 & 0.452 \\
\hline Season (early dry) & -0.024 & 0.006 & -0.036 & -0.012 & -0.027 & -0.022 & 9.432 & 1 & 0.002 \\
\hline \multicolumn{10}{|c|}{$\begin{array}{l}\text { a Minimum and maximum of model estimates obtained when dropping levels of random effects one at a time } \\
\text { b Results of a likelihood ratio test comparing the full model with a reduced model lacking the respective term } \\
\text { "Not shown as not having a meaningful interpretation. See footnotes of Table S1 for details. } \\
\text { d z-transformed, mean and SD of the original values were } 6.387 \text { and } 2.082 \text {, respectively } \\
\text { "Manually dummy-coded with the year "2016", "females" and "late dry season" being the reference categories }\end{array}$} \\
\hline
\end{tabular}

Altogether, grooming network analyses in our study population yielded contrasting patterns compared to a study in a differen population (Perofsky et al., 2017). Based on weighted grooming networks, we found individuals in groups with higher connectedness and group members that groom each other more to share less similar microbiomes. Moreover, no correlation was found between individual network centrality and microbial diversity. While no associations between affiliative behaviors and microbial diversity have been reported before and are also congruent with our other results (see above), the findings that groups and dyads which engage more in grooming share less similar microbiomes seem counterintuitive. We doubt that our data captured all relevant grooming connections within groups, despite our large data set containing hundreds of hours of behavioural observations. Grooming behaviour in sifakas is relatively rare and short (Lewis, 2010) and dense observation schedules are required to collect sufficient data to enable the construction of reliable grooming networks. Yet, such sampling effort is difficult to implement in wild populations. Weighted grooming networks, which are based on only a few minutes of dyadic interactions observed over months, are prone to contain incomplete information about animals' social connectivity. We therefore believe that grooming network statistics - if they are not based on intense observation schedules - cannot provide meaningful answers to questions addressing the impact of social interactions on horizontal bacterial transmissions in Verreaux's sifakas. 


\section{d. Leave and fruit intake per group per season}

We applied two binomial models with beta error distribution structures and a logit link function using the glmmTMB package (version 0.2.3) (Brooks et al., 2017) to examine variation in leave and fruit consumption between group. We used mean intake rates on leaves or fruits per individual per season as response variables, group ID and season (early dry or late dry) as predictors variables and group and animal ID as random effect. P-values for individual effects were based on likelihood ratio tests comparing the full with the respective null models using the drop1 function (Barr et al., 2013). We encountered no issues when checking for model stability and overdispersion and assessed confidence intervals with the confint function of the glmmTMB package.

Both models were significant (Leaves: $\chi^{2}=24.702, \mathrm{df}=7, \mathrm{p}>0.001$; Fruits: $\chi^{2}=32.118, \mathrm{df}=7, \mathrm{p}>0.001$ ) (Tables S23 and S24). Intake rates of both, leaves and fruits, were higher during the early dry season, when more food was available (Kappeler and Fichtel, 2012; Rudolph et al., 2019), indicating that sifakas increase their intake of other food items, e.g. flowers, barks or seeds (Koch et al., 2017) during the late dry season. Groups did not differ significantly in their leave and fruit consumption (Figure S8).

Table S23 Variation in leave consumption between groups and seasons in Verreaux's sifakas; results of the full model (GLMM; Nobservations $=133, \mathrm{~N}_{\text {Groups }}=7, \mathrm{~N}_{\mathrm{ID}}=41$ ).

\begin{tabular}{|c|c|c|c|c|c|c|c|c|c|c|}
\hline Term & & Est & SE & Lower $\mathrm{Cl}$ & Upper Cl & $\operatorname{Min}^{a}$ & $\mathrm{Max}^{\mathrm{a}}$ & $\chi^{2 b}$ & $d f$ & $P$ \\
\hline (Intercept) & & -0.032 & 0.211 & -0.446 & 0.383 & -0.260 & 0.042 & c & c & c \\
\hline \multirow[t]{6}{*}{ Group $^{\mathrm{e}}$} & $\mathrm{F}$ & -0.125 & 0.251 & -0.617 & 0.368 & -0.225 & 0.007 & $3.857^{d}$ & $6^{d}$ & $0.696^{d}$ \\
\hline & F1 & -0.273 & 0.343 & -0.946 & 0.400 & -0.374 & -0.141 & c & c & c \\
\hline & G & -0.325 & 0.292 & -0.897 & 0.246 & -0.426 & -0.195 & c & c & c \\
\hline & J & -0.015 & 0.279 & -0.562 & 0.531 & -0.254 & 0.133 & c & c & c \\
\hline & L & -0.478 & 0.298 & -1.062 & 0.105 & -0.283 & 0.045 & c & c & c \\
\hline & $M$ & -0.086 & 0.340 & -0.753 & 0.580 & -0.225 & 0.007 & c & c & c \\
\hline \multicolumn{2}{|c|}{ Season (early dry) } & 0.721 & 0.153 & 0.422 & 1.020 & 0.599 & 0.930 & $20.688^{d}$ & $1^{d}$ & $>0.001^{d}$ \\
\hline \multicolumn{11}{|c|}{$\begin{array}{l}\text { a Minimum and maximum of model estimates obtained when dropping levels of random effects one at a time } \\
\text { b Results of a likelihood ratio test comparing the full model with a reduced model lacking the respective term } \\
\text { "Not shown as not having a meaningful interpretation. See footnotes of Table S1 for details. } \\
\text { d Values refer to the overall test of the effect of the predictors ("Group", "Season"), not the specific level indicated in the respective row } \\
\text { e Manually dummy-coded with group "E" and season "late dry" being the reference categories }\end{array}$} \\
\hline
\end{tabular}


Table S24 Variation in fruit consumption between groups and seasons in Verreaux's sifakas; results of the full model (GLMM; Nobservations $=133, N_{\text {Groups }}=7, N_{I D}=41$.

\begin{tabular}{|c|c|c|c|c|c|c|c|c|c|c|}
\hline Term & & Est & SE & Lower $\mathrm{Cl}$ & Upper Cl & $\operatorname{Min}^{\mathrm{a}}$ & $\mathrm{Max}^{\mathrm{a}}$ & $\chi^{2 b}$ & $d f$ & $P$ \\
\hline (Intercept) & & -2.114 & 0.236 & -2.577 & -1.650 & -2.605 & -2.027 & c & c & c \\
\hline \multirow[t]{6}{*}{ Group ${ }^{e}$} & $\mathrm{~F}$ & -0.516 & 0.272 & -1.051 & 0.018 & -0.640 & -0.377 & $10.431^{d}$ & $6^{d}$ & $0.108^{d}$ \\
\hline & F1 & -0.059 & 0.370 & -0.783 & 0.666 & -0.169 & 0.453 & c & c & c \\
\hline & G & 0.163 & 0.301 & -0.428 & 0.754 & 0.062 & 0.670 & c & c & c \\
\hline & J & -0.185 & 0.287 & -0.748 & 0.378 & -0.338 & 0.324 & c & c & c \\
\hline & L & 0.445 & 0.316 & -0.175 & 1.064 & 0.326 & 0.963 & c & c & c \\
\hline & $M$ & -0.305 & 0.385 & -1.060 & 0.450 & -0.461 & 0.217 & c & c & c \\
\hline \multicolumn{2}{|c|}{ Season (early dry) } & 0.902 & 0.178 & 0.552 & 1.251 & 0.750 & 1.150 & $24.231^{d}$ & $1^{d}$ & $>0.001^{d}$ \\
\hline \multicolumn{11}{|c|}{$\begin{array}{l}\text { a Minimum and maximum of model estimates obtained when dropping levels of random effects one at a time } \\
\text { b Results of a likelihood ratio test comparing the full model with a reduced model lacking the respective term } \\
\text { " Not shown as not having a meaningful interpretation. See footnotes of Table S1 for details. } \\
\text { d Values refer to the overall test of the effect of the predictors ("Group", "Season"), not the specific level indicated in the respective row } \\
\text { e Manually dummy-coded with group "E" and season "early dry" being the reference categories }\end{array}$} \\
\hline
\end{tabular}

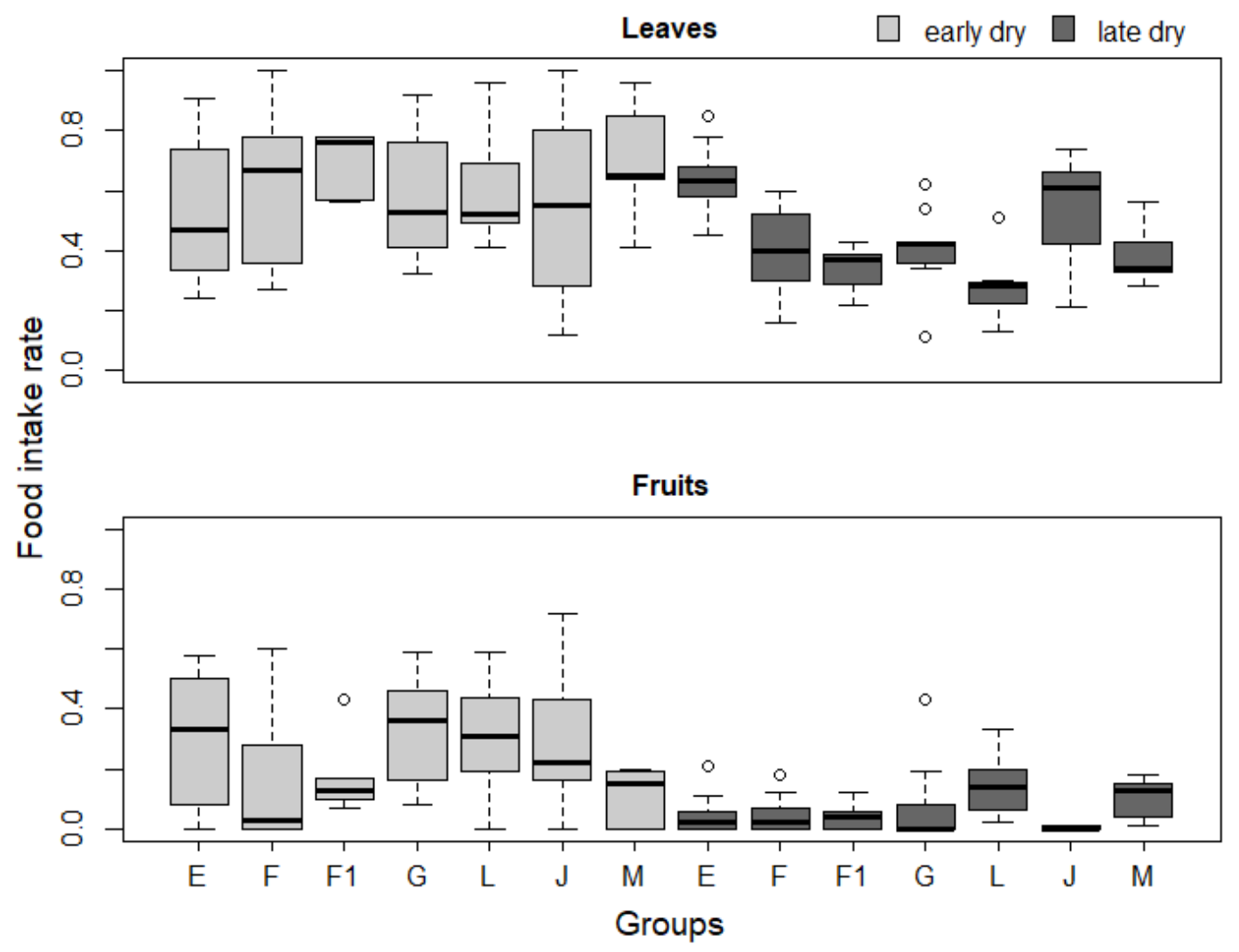

Figure S8 Average leave and fruit intake rates of Verreaux's sifaka groups during the early and late dry season. Boxplots comprise mean individual consumption rates per season with lines indicating median, upper and lower quartiles. Whiskers indicate $+/$ - 1.5 interquartile ranges and small circles beyond whiskers indicate outliers 


\section{Chapter 5}

\section{General Discussion}

\subsection{A Brief Summary}

Evidence from various scientific disciplines is accumulating that social behaviours crucially impact human and animal health. However, little is known about the proximate mechanisms underlying the socialityhealth link. One reason for this limited understanding is the low number of longitudinal studies that systematically examined this relationship in wild populations (Kappeler et al., 2015; Silk, 2014). Here, I add needed data to this field of research by studying links between sociality and indicators of health in wild Verreaux's sifakas for two consecutive years. I examined various aspects of Verreaux's sifakas' group life, i.e. group size, group membership, rank, affiliative and agonistic interactions, and their potential associations with parasite infections, energy budgets and microbial communities. In the following, I will summarise the main findings of my thesis.

\section{Study I-Group size, Health and Ecology}

In the first study, I tested predictions of the ecological constraints and the optimal group size hypothesis, which link group size to condition and health. Group size had neither impact on groups' ranging patterns and daily activities nor on individual glucocorticoid metabolite concentrations or parasite richness, while seasonal variation in food availability and temperature differences affected the majority of variables. The study does not support the tested hypotheses and I conclude that there is no optimal group size in Verreaux's sifakas, presumably because group sizes of this species remain below the upper, ecologically "optimal" limits. It appears, that most folivorous mammals, like Verreaux's sifakas, are constrained in group size by social rather than ecological factors.

Study II - Dynamics and Determinants of Individual fGCM Concentrations

In the second study, I examined the potential impact of social and ecological factors on concentrations of fGCMs. Measures of fGCMs were correlated to seasonal variation in temperature, fruit availability and intake. Moreover, they were elevated during premating periods in males and gestation in females, while there was no link with agonistic or affiliative interactions, adult sex ratios, vigilance or scratch rates. Effects of male rank on fGCMs vary across years, indicating that energetic constraints on male rank may depend on extrinsic factors like annual variation of their social and ecological environments. I show that measures of glucocorticoids constitute valuable tools for studying energetic burdens of ecological and reproductive challenges in wild populations, while they seem to be insufficient indicators for immediate consequences of social and non-social behaviours that are not directly linked to energy budgets. 


\section{Study III - Exploring Between-Group Variation in Gut Microbial Communities}

In the third study, I investigated the causes for between-group variation on gut microbial communities by correlating measures of gut microbial composition and diversity with data on social interactions, maternal relatedness, diet, habitat structure, habitat overlap and seasonality. Group membership and maternal relatedness, but not social interactions, affected dyadic gut microbial similarity, whereas none of the environmental predictors, i.e. habitat overlap, habitat dissimilarity and diet, explained variation in gut microbiomes between groups. Environmental factors seem to determine the general set-up of gut microbial compositions on a species- or population level, but finer-scaled environmental differences between local groups do not have detectable effects on gut communities. Kinship plays a large role for between-group variation in microbial communities however, other factors must also contribute to this effect since unrelated individuals had more similar microbiota with group members than relatives living in different groups. Rare physical contact between members of different groups and frequent physical contact among members of the same group may contribute to the here found group membership effects, however, more research is required to confirm this assumption.

In summary, most of the here found behavioural and physiological differences between groups or individuals were not affected by social variables but could be explained by seasonal changes in animals' physical environments. In light of the two main costs of sociality, i.e. a) social transmission of pathogens and microbes, and b) increased susceptibility to diseases owing to social stress (Kappeler et al., 2015), I found some evidence for the former but no indication for the latter. Concerning a), I could show that group living had a clear impact on individuals' gut microbiota, which may have important implications for their health, however, state-of-the-art knowledge on host-microbiota interactions remains too poor to derive reliable conclusions about health-effects. As for b), I found no association between social interactions and competition within or between groups with individual fGCM concentrations and parasite infections. The results of my thesis, therefore, indicate that health-related consequences of different aspects of group living in Verreaux's sifakas are limited to effects caused by social proximity but not social interactions with group members. A closer look at Verreaux's sifakas' behavioural ecology might explain some of these findings.

\subsection{Limited Health-Related Consequences of Sociality in Verreaux's Sifakas}

\section{- A Consequence of the Mysterious "Lemur Syndrome"?}

Primates show considerable diversity and flexibility in their social organisations, ranging from solitaryand pair-living up to the formation of multi-level societies (Silk and Kappeler, 2017). Socioecological theory has derived general frameworks which provide major contributions to understanding variation in anthropoid primates' social systems (Clutton-Brock and Harvey, 1977; Crook and Gartlan, 1966; Emlen 
and Oring, 1977; Kappeler and van Schaik, 2002; Koenig et al., 2013). However, lemur societies have puzzled scientists for decades with the so-called "lemur syndrome" - an array of patterns that are not found in anthropoid primates and have proven difficult to be explained by socioecological models (Eberle and Kappeler, 2004; Kappeler and Fichtel, 2015; Kappeler and Schäffler, 2007). This phenomenon comprises behavioural, morphological and ecological traits, including even adult sex ratios (Kappeler et al., 2009b), female dominance (Jolly, 1984; van Schaik and Kappeler, 1996), and the lack of male-sexual dimorphism regardless of mating system (Kappeler and Schäffler, 2007). Previous attempts to understand the evolution of the lemur syndrome proposed adaptations to Madagascar's harsh and unpredictable environment (Dewar and Richard, 2007; Kappeler et al., 2019; Wright, 1999; but see Federman et al., 2017), relaxed male mating competition (Hrdy, 2009; Kappeler, 1993) or a combination of evolutionary and developmental processes based on chronic maternal stress (Kappeler and Fichtel, 2015) as possible explanations. Thus, lemurs express unique patterns in social and sexual behaviours, which might explain the relatively low health-related costs and benefits of their social life.

\section{"Pacifism" Reduces Health-Related Costs of Sociality}

Social interactions play a key role for individual health (Kappeler et al., 2015; Ostner and Schülke, 2018) as they can mediate physiological stress responses (Raulo and Dantzer, 2018), enhance risks of pathogen transmission (Poulin, 2018) and facilitate the exchange of microbial-mediated pathogen resistance (Ezenwa et al., 2016). Affiliative and agonistic interactions are part of daily life in social groups and enable animals to establish and maintain social relationships, to determine dominance positions and to gain access to mating opportunities. However, compared to most anthropoid primates, lemurs devote little time to social interactions (Erhart and Overdorff, 2008; Rasolonjatovo and Irwin, 2019; Wheeler et al., 2013), presumably affecting the health-consequences of their social life.

Agonistic interactions are especially important for establishing social hierarchies (Drews, 1993; Wheeler et al., 2013). In Verreaux's sifakas, hierarchies are linear with adult females at the highest positions (Kappeler and Schäffler, 2007; Kraus et al., 1999). Hierarchical relationships in this species seem to be more relaxed and tolerant compared to other species (Norscia and Palagi, 2015). Even though agonistic interactions are used to determine ranks within groups, they are very rare (study II; Kappeler et al., 2009; Wheeler et al., 2013), seldom result in severe wounding and there are no reports of lethal aggression during fights in this species (Benadi et al., 2008). Also, lemurs in general use unidirectional vocal or olfactory signals to settle and assure their relationships (Benadi et al., 2008; Gould and Overdorff, 2002; Kappeler, 1998; Kulahci et al., 2015). In Verreaux's sifakas, rates of submissive chatter vocalisations are positively linked to grooming rates and negatively linked to rates of aggression (Lewis, 2019). Similarly, they use scent-marking, i.e. rubbing their anogenital and, in the case of males, sternal scent glands against the bark of trees, to regulate their relationships and communicate their presence (Benadi et al., 2008; 
Lewis, 2005). Hence, the use of non-physical communication may mitigate the necessity of agonistic interactions (Flack and Waal, 2007).

Moreover, dominant males mate-guard females (Brockman, 1999; Mass et al., 2009) but mateguarding is not associated with increased aggression, body size or weaponry (Lawler et al., 2005; Mass et al., 2009). Instead, males seem to rely on morphological traits which provide advantages in form of speed and agility during agonistic chases to increase reproductive success (Lawler et al., 2005). Female competition is ubiquitous - and a likely cause for Verreaux's sifakas' small group sizes (Kappeler and Schäffler, 2007; Steenbeek and van Schaik, 2001) - but relatively peaceful, since evictions have never been observed in this study population (Kappeler and Fichtel, 2012). Additionally, food competition is expected to be low due to sifakas' small group sizes and mainly folivorous diet (Janson and Goldsmith, 1995; Koenig, 2002). All these factors indicate that Verreaux's sifakas do not require frequent aggressive behaviours to compete with their group mates, because competition is low and they use other forms of communication to convey their social status and relationships and thus, avoid costly physical conflict.

Similarly, intergroup aggression in Verreaux's sifakas is moderate (Benadi et al., 2008; Koch et al., 2016b). Sifakas are territorial and defend their home ranges with both sexes participating in encounters (Jolly, 1966; Kappeler et al., 2009b; Koch et al., 2016b). Encounters between neighbouring groups are common and mostly occur at shared feeding sites in overlapping areas of their home ranges (Benadi et al., 2008; Koch et al., 2016a). Physical fights are, however, rare and animals usually use chases, stares, growls and scent marks during encounters (Brockman et al., 1998; Norscia et al., 2009). Moreover, the risk of group takeovers by external males is relatively low with 0.6 population-wide takeovers per year (Kappeler et al., 2009b) and infanticide is rare (Lewis et al., 2003b). A prior study in this population found that groups will not change their ranging and activity patterns when approaching overlapping home range areas, where the likelihood of encounters increases (Benadi et al., 2008). Hence, the risk of intergroup encounters does not exert changes to their behaviours - potentially because the costs of encounters are usually low.

Low rates of aggressive conflicts may also derive from Verreaux's sifakas' arboreal lifestyle. Arboreal species are characterised by less frequent agonistic interactions than terrestrial species (DeVore, 1963; Wheeler et al., 2013). This could be a result of the generally smaller group sizes found in arboreal species (Clutton-Brock and Harvey, 1977) in which competition over resources is considered reduced (Janson and Goldsmith, 1995; Janson and van Schaik, 1988). Another suggestion was that agonistic interactions on arboreal substrates involve higher energetic costs and are more risky in light of the danger of injury from falling (Hill and Okayasu, 1995). The complexity of the arboreal environment may also prevent animals from engaging in physical conflict, simply because it is too difficult to reach the opponents (Wheeler et al., 2013). 
Altogether, Verreaux's sifakas mainly pursue non-violent, low-risk strategies to cope with interand intragroup competition. Rare aggressive interactions might derive from low within- and betweengroup competition per se and the high energetic costs and risks associated with physical conflict. Here, fGCM concentrations were not linked to aggressive behaviours (study II), indicating that Verreaux's sifakas' agonistic behaviours do not inflict high energetic constraints on individuals, and might thus not result in significant health-related consequences.

\section{Moderate Affiliation = Moderate Health-Consequences?}

Allogrooming is one of the most common affiliative behaviours in primates (Dunbar, 1991; Russell and Phelps, 2013) and other mammals (Kutsukake and Clutton-Brock, 2006; Stopka and Graciasová, 2001). This behaviour most likely evolved for hygienic functions, i.e. the removal of dirt and ectoparasites from hard-to-reach areas (Grueter et al., 2013; Hutchins and Barash, 1976). Allogrooming can also provide other health-related benefits, in terms of decreased heart rates (Aureli, 1997; Boccia et al., 1989) and increased insulation of animals' furs providing thermal benefits (McFarland et al., 2016), and it is associated with changes in concentrations of GCs (Crockford et al., 2008; Wittig et al., 2008) and oxytocin (Benítez et al., 2018). Frequent grooming of conspecifics serves the formation and maintenance of social relationships as shown in studies across different taxa (Kutsukake and Clutton-Brock, 2006; Madden and Clutton-Brock, 2009; Radford and Du Plessis, 2006; Sánchez-Villagra et al., 1998). In contrast to anthropoid primates, lemurs groom each other orally with so-called "toothcombs", a modification of the lower incisors and canines (Barton, 1987; Eaglen, 1980), which might facilitate the transmission of pathogens (Clough et al., 2010). On the other hand, allogrooming occurs at much lower rates in lemurs than in other primates (Sussman and Garber, 2004), which might limit opportunities for social transmission.

Several studies have established that Verreaux's sifakas exhibit low intestinal parasite diversity (Loudon and Sauther, 2013; Muehlenbein et al., 2003; Springer and Kappeler, 2016) and here, I found the same pattern (study I). Sifakas' arboreality and folivory might protect them from parasites that are transmitted via soil or use invertebrates as intermediate hosts (Loudon and Sauther, 2013; Springer and Kappeler, 2016). Animals may also utilize self-medication via the ingestion of certain plant species and soils (Huffman, 1997; Semel et al., 2019). Self-medicating behaviours are common in primates and, amongst other beneficial effects, may enhance parasite immunity (Carrai et al., 2003; Huffman, 2003; Peckre et al., 2018). Moreover, Gl parasite diversity may also be inhibited if there are limited opportunities for social transmission between animal hosts. Allogrooming can function as an important driver for the transmission of Gl nematodes (Friant et al., 2016; Maclntosh et al., 2012). However, Verreaux's sifakas devote little time to affiliative interactions, like allogrooming (studies II \& III; Lewis, 2010, 2008), which could further contribute to their low nematode diversity. 
It should be noted though, that the impact of social transmission of different microorganisms depends on their biological characteristics and modes of transmission. For example, the transmission routes of lemur-parasitising members of Trichostronylidae - which were present in all individuals (study I; Springer and Kappeler, 2016) - are still unknown (Irwin and Raharison, 2009). Larvae of Trichostrongylidae usually hatch in the environment and need several days to develop into an infective stage (Bush et al., 2001). Yet, Verreaux's sifakas are arboreal and spent very little time on the ground, providing few chances for picking up larvae. Faecal-oral transmission of this parasite, by which infective larvae cling to animals' furs and get swallowed by groomers during grooming bouts, might explain its high prevalence (Springer and Kappeler, 2016). Similarly, there are indications for social transmission of microbes in this species, as group members share the same E. coli strains (Springer et al., 2016) and groups harbour distinct gut bacterial microbiota (study III; Perofsky et al., 2017; Springer et al., 2017). However, neither the routes transmission nor the conditions necessary for the spread of microorganisms are well understood (Brito et al., 2019; Kuthyar et al., 2019; Robinson et al., 2019; Webster et al., 2017). Successful transmission might crucially depend on the duration of social contacts or external environmental conditions, e.g. temperature or humidity, (Browne et al., 2017; Ferguson and Signoretto, 2011; Rossanigo and Gruner, 1995; Sutherst and Bourne, 2006), which is why future research should address these important issues.

Ectoparasites usually require direct host-to-host contact for transmission and can be removed via auto- and allogrooming (Akinyi et al., 2013; Hawlena et al., 2007). Verreaux's sifakas harbour different ectoparasitic species, including mites, lice and ticks (Rasambainarivo et al., 2014; Springer, 2015). Prior research in this study population found ectoparasites mainly in regions that are difficult to groom, like armpits, ears or skinfolds, indicating that auto- or allogrooming may have removed parasites from other body parts (Springer, 2015). However, the same study did not detect links between allogrooming or body contact rates and the probability of ectoparasite infections. Thus, Verreaux's sifakas' short and relatively few allogrooming bouts may alleviate groomees from some ectoparasitic loads but are not sufficient enough to reduce ectoparasitic infection probabilities. Behavioural observations in this species are conducted during the day since sifakas are exclusively diurnal (Erkert and Kappeler, 2004). Yet, inclusions of body contact rates during the night might reveal new insights on the parasite spread. Especially during cold nights, group members huddle together for thermoregulation, which may provide sufficient host-tohost contact for ectoparasitic transmission throughout the groups.

Overall, allogrooming seems to have limited effects on sifakas' hygiene and health, but carries out important social functions as it helps maintaining group cohesion (Lehmann et al., 2007), enables sifakas to reassure their rank positions and relationships (Lewis, 2010), facilitates male mating success (Norscia et al., 2009) and is used by immigrants to negotiate group membership (Lewis, 2008). Considering its importance for sifakas' social life, why do they invest so little time in this behaviour? 


\section{Are Sifakas Too Busy or Too Scared to Groom?}

\section{- Impacts of Folivory and Predation Risk}

Affiliative interactions can be time-consuming (Caraco, 1979; Korstjens et al., 2006; Pollard and Blumstein, 2008). Time is a finite resource and how much of this resource can be allocated to sociality depends on animals' habitats, diets and life history strategies (Dunbar et al., 2009; Marty et al., 2019). Folivorous species require longer resting times to allow for the digestion of leaf material (Fleagle, 2013; Korstjens et al., 2010; Pollard and Blumstein, 2008), resulting in less time available for social interactions (Kavana et al., 2015; Saj et al., 2007). Verreaux's sifakas spent the largest proportions of their days resting (47\% study I) and foraging (45\% to 47\% study I \& in Koch et al., 2017). During the study period, animals' foraging rates decreased slightly during the dry season and the time spent resting increased. However, in a prior study, the opposite patterns were found, yet, also here differences were small (Koch et al., 2017). Overall, feeding and resting durations in this species seem to be fairly stable throughout the year. Allogrooming rates in Verreaux's sifakas are unaffected by seasonality as well (Lewis, 2010). Over the course of the dry season, Verreaux's sifakas lose significant amounts of body mass and fat due to the increased energetic constraints caused by decreased food availability (Lewis and Kappeler, 2005) and higher needs of thermoregulation during cold nights. To cope with the challenges of the harsh dry season, sifakas store nutrients and fat, which need to be accumulated during the wet season (Koch et al., 2017). Such storing capacities have also been described in co-resident species, i.e. folivorous red-tailed sportive lemurs (Lepilemur ruficaudatus) (Ganzhorn, 2002), grey mouse lemurs (Microcebus murinus) (Schmid and Speakman, 2000) and fat-tailed dwarf lemurs (Cheirogaleus medius) (Dausmann, 2014). Verreaux's sifakas might, therefore, be obliged to spend the majority of their time foraging and resting throughout the year and regardless of whether food availability is high or low, in order to fulfil the energetic requirements of their challenging environment. Animals' may thus simply be too "busy" and unable to afford the time for extended sessions of allogrooming.

Additionally, grooming activities distract animals from being vigilant (Maestripieri, 1993) which can increase predation risks (Cords, 1995; Hart et al., 1992; Mooring and Hart, 1995). Oral grooming, in contrast to manual grooming, might put the individuals even more at risk, as they are not just distracted from their surroundings but their field of vision during grooming is also considerably impaired by the groomees' body. Predation pressure is generally high in lemurs since they represent the largest and most abundant mammals on Madagascar (Scheumann et al., 2007; Wright, 1998). In fact, the main cause of death in Verreaux's sifakas of Kirindy Forest, but also in other sifaka species, is fossa predation (Cryptoprocta ferox) (Kappeler and Fichtel, 2012; Pochron et al., 2004; Wright et al., 1997). Therefore, allogrooming, next to being time-consuming, also poses a source of danger for individuals and might thus not be conducted longer than necessary for maintaining social cohesion. 


\section{Energetic Constraints Shape Verreaux's Sifakas' Social Life}

During the 2-year study period, all Verreaux's sifakas of the study population seemed to be "healthy" in the sense of the applied definition by Huber et al. (2011) (i.e. health is "the ability to adapt and to selfmanage' when facing physical, mental, and social challenges") and no individual or group stood out in its behavioural patterns or physiological measures. All animals were able to do so despite living in a harsh and unpredictable environment, where they have to maintain core body temperatures, fight parasitic infections, find enough food and produce offspring. These requirements are energetically costly and might compromise animals' condition, especially in habitats where resources are limited. In comparison to haplorhines, lemurs have lower basal metabolic rates (Genoud, 2002; Simmen et al., 2010), which suggests that they rely on thermoregulatory behaviours to deal with the thermal stress during colder seasons. Smaller lemurs (< 200g body mass) usually enter daily torpor or hibernate (Dausmann, 2014), whereas larger lemurs will sun-bask and perform "social thermoregulation" by huddling together (Eppley et al., 2017a; Morland, 1993; Ostner, 2002). Verreaux's sifakas had increased fGCMs concentrations during the colder dry season in both study years (study II). This result supports the notion of increased energetic constraints during this time, which sifakas counter with reduced activity (study I; Erkert and Kappeler, 2004) and group-huddling.

Altogether, Verreaux's sifakas constantly face ecological challenges in form of food deprivation, high predation risks and thermal stress, which they manage to overcome by living in permanent association with conspecifics. However, their energetically demanding lifestyle and habitat appear to mainly constrain them from physical social interactions that go further beyond the maintenance of group cohesion. Health-related consequences of sociality are often related to increased stress through social conflict or isolation, which can increase susceptibility to diseases (Cohen, 2004; Kappeler et al., 2015) and to the increased transmission of pathogens (Freeland, 1976) and microbes (Lombardo, 2008; Turnbaugh et al., 2009). Here, I did not detect an effect of social relationships on animals' physiological conditions (study II \& III), likely because of sifakas' low interaction rates and relaxed competition. Proximity to group members, however, was linked to gut microbial similarity (study III) and might facilitate the transmission of certain endo- and ectoparasites (study I; Springer and Kappeler, 2016).

Overall, I did not observe any signs of sickness or impaired health among the individuals, but it should be considered that my findings are based on non-invasive physiological parameters derived from faecal samples. Such measures provide valuable insights into animals' metabolic functions and general condition but cannot keep up with the accuracy and comprehensiveness of clinical studies. My study provides new evidence for the interplay of sociality, ecology and health in an endangered primate species but also underlies the typical constraints and limitations of field research, which I will briefly discuss in the following section. 


\subsection{Measuring Health in the Wild}

\section{Strengths \& Limitations}

Wildlife studies are important for investigations of the sociality-health nexus as they account for naturally occurring variation in aspects of sociality within an ecologically meaningful context. The inclusion and consideration of ecological and potentially confounding factors in my analyses proved very helpful for the interpretation of my results. For example, I provided evidence that $\mathrm{fGCM}$ concentrations are poor indicators for assessments of social stress in the wild, whereas they offer valuable insights into the energetic constraints animals face in their habitats, e.g. through changes in temperatures and food availability or gestation (study II). Due to the 2-year duration of the study, I could show recurrence of seasonal patterns in physiological adaptations and challenges. Next to the seasonal variation in fGCMs, I found nematodes of the family Oxyuridae to only occur during the wet season in both study years (study I) and detected consistent shifts in the abundance of certain gut bacterial phyla during the transition from the early to the late dry season (study III).

All physiological measures I used to investigate animals' health are derived from faecal samples, which are commonly utilised in field studies since they can be collected easily, non-invasively and repeatedly from the same individual (Touma and Palme, 2005). Nevertheless, faecal samples can only provide a glimpse on animals' physiological condition and there exist numerous confounding factors that can affect the results of their analyses.

\section{Hormones}

Faecal hormone metabolite (FHM) analyses enable the assessment of various endocrine functions and reflect the accumulated production rate of hormones over several hours (Touma and Palme, 2005). Endocrinological measures derived from faecal samples are mostly restricted to thyroid and steroid hormones, either because metabolites of other hormones are not secreted into faeces or they are too degraded for reliable detection (Behringer and Deschner, 2017; Pribbenow et al., 2017). There are numerous confounding factors which can influence FHM analyses, including study species, sex, diet, age or prenatal stress (Palme, 2019). Similarly, contamination with urine, water or blood can falsify measurements (Bahr et al., 2000; Behringer and Deschner, 2017). One of the most commonly utilised methods for measuring FHMs are immunoassays, which were also applied in this study. Importantly, there remains uncertainty about the metabolites which are picked up and measured with these assays due to the risk of cross-reactivity of the antibody (Goymann, 2012; Touma and Palme, 2005). Until these uncertainties are eliminated, results and patterns of FHMs derived from immunoassays should be interpreted with caution. Nevertheless, if methods for FHM analyses are correctly selected, validated and applied they can provide powerful tools for monitoring hormonal activity in wildlife. 


\section{Intestinal Parasites}

The most commonly used methods to non-invasively quantify intestinal parasite burdens are estimations of prevalence and intensity via microscopy. However, identification and distinction of certain parasite species can be difficult due to morphological similarities (Polderman and Blotkamp, 1995). Here, I applied a metabarcoding approach by which I assessed parasite infestations using next-generation sequencing of 18 rRNA genes (Hadziavdic et al., 2014). To date, only a handful of studies have this approach to noninvasively assess intestinal parasites in animals (Avramenko et al., 2015; Srivathsan et al., 2016; Tanaka et al., 2014; Wimmer et al., 2004). Metabarcoding can facilitate differentiation among closely related species and constitutes a faster and more precise method in comparison to traditional analyses (Aivelo et al., 2018). Still, there is room for improvement since only a minority of parasite species is represented in reference databases and therefore detectable (but see Aivelo and Medlar, 2017; Wilson et al., 2011). My results on intestinal nematode infections in Verreaux's sifakas support the findings of prior studies (Loudon and Sauther, 2013; Springer and Kappeler, 2016), yet, more studies are required to complement the databases and reveal potentially unknown parasites.

\section{Gut microbial analyses}

There is an increasing number of wildlife studies investigating links between sociality and gut microbiota (Amato, 2016; Theis et al., 2012; Tung et al., 2015; Youngblut et al., 2019). However, even though our understanding of microbial communities has greatly improved within the past decade, we still know very little about microbiomes. To date, a large diversity of microorganisms remains completely unknown occasionally referred to as "microbial dark matter" (Bernard et al., 2018; Thomas and Segata, 2019) -, we are just beginning to identify the factors that influence gut microbiota and our insights into the functions and transmission routes of bacteria are limited (Browne et al., 2017; Moeller et al., 2018; Rinninella et al., 2019). Thus, there is much more work required before we can fully understand the impact of gut microbial communities on animal and human health. More basic research should be implemented in future research (e.g. by examining hourly and daily shifts in microbial compositions) and future studies need to acknowledge the limited insights and myriads of unanswered question concerning this field of research to avoid mis- and over-interpretation.

\section{Future directions}

Here, I provide comprehensive research on associations between different aspects of group living and measures of fGCM concentrations, parasite richness and gut microbiota in wild Verreaux's sifakas. However, the links between sociality and health are complex and there remain many unanswered questions that need to be addressed in future studies. A well-functioning immune system is necessary for pathogen resistance and ultimately for survival. There exist a range of non-invasive immune markers derived from faecal or urinary samples (Higham et al., 2015), which could be implemented in future 
research and would provide valuable information on individual immune responses, especially in combination with endocrinological parameters and assessments of gut microbial communities. Further investigations in this species should also relate measures of health to long-term data on survival and reproductive success to assess the fitness consequences of individual variation in health that derived from the evolution of group living.

\subsection{Conclusions}

\section{A Small Step Closer Towards Understanding the Evolution of Sociality}

The transition from solitary to group living yielded various new health-related costs and benefits. However, the magnitude of these health consequences may depend on species-specific aspects of their sociality, i.e. social organisation, social structure and mating system (Kappeler and van Schaik, 2002). Individuals living in highly competitive societies may be exposed to higher rates of agonistic and affiliative interactions, which represent a source for variation in physiological or psychological stress and can increase opportunities for transmission of pathogens and microbes. On the contrary, in species with less competitive regimes and low interaction rates, health-consequences of sociality might be attenuated. Here, I only found few implications for health-related consequences of group living in Verreaux's sifakas, potentially reflecting the species' low competition within and between groups, little investment in social relationships and avoidance of physical aggression. Importantly, I found strong impacts of environmental factors on animals' behaviours and physiology which might have obscured patterns of the sociality-health nexus, underlying the importance of examining the nexus within an ecologically meaningful context.

There is still little understanding of the complex and interrelated factors that contribute to the sociality-health link and its consequences for individual fitness. Future research should comprise of comprehensive wildlife studies in species with different social systems. In light of the rapid advances on applications of non-invasive techniques to field studies and the constant progress in molecular and statistical methods, upcoming research holds great potential for providing major contributions to the understanding of the co-evolutionary dynamics and factors that shaped the evolution of sociality. 


\section{References}

Abbott, D.H., Keverne, E.B., Bercovitch, F.B., Shively, C.A., Mendoza, S.P., Saltzman, W., Snowdon, C.T., Ziegler, T.E., Banjevic, M., Garland Jr., T., Sapolsky, R.M., 2003. Are subordinates always stressed? a comparative analysis of rank differences in cortisol levels among primates. Hormones and Behavior 43, 67-82. https://doi.org/10.1016/S0018506X(02)00037-5

Abt, M.C., Pamer, E.G., 2014. Commensal bacteria mediated defenses against pathogens. Current Opinion in Immunology 29, 16-22. https://doi.org/10.1016/j.coi.2014.03.003

Adkins-Regan, E., 2005. Hormones and animal social behavior. Princeton University Press.

Ainsworth, T.D., Krause, L., Bridge, T., Torda, G., Raina, J.-B., Zakrzewski, M., Gates, R.D., Padilla-Gamiño, J.L., Spalding, H.L., Smith, C., Woolsey, E.S., Bourne, D.G., Bongaerts, P., Hoegh-Guldberg, O., Leggat, W., 2015. The coral core microbiome identifies rare bacterial taxa as ubiquitous endosymbionts. ISME J 9, 2261-2274. https://doi.org/10.1038/ismej.2015.39

Aivelo, T., Medlar, A., 2017. Opportunities and challenges in metabarcoding approaches for helminth community identification in wild mammals. Parasitology 145, 608-621. https://doi.org/10.1017/\$0031182017000610

Aivelo, T., Medlar, A., Löytynoja, A., Laakkonen, J., Jernvall, J., 2018. Metabarcoding gastrointestinal nematodes in sympatric endemic and nonendemic species in Ranomafana National Park, Madagascar. Int J Primatol 39, 49-64. https://doi.org/10.1007/s10764-017-0010-x

Akinyi, M.Y., Tung, J., Jeneby, M., Patel, N.B., Altmann, J., Alberts, S.C., 2013. Role of grooming in reducing tick load in wild baboons (Papio cynocephalus). Animal Behaviour 85, 559-568. https://doi.org/10.1016/j.anbehav.2012.12.012

Alexander, R.D., 1974. The evolution of social behavior. Annual Review of Ecology and Systematics 5, 325-383.

Allan, A.T.L., Hill, R.A., 2018. What have we been looking at? A call for consistency in studies of primate vigilance. American Journal of Physical Anthropology 165, 4-22. https://doi.org/10.1002/ajpa.23381

Alm, M., Holm, L., Tauson, R., Wall, H., 2014. Corticosterone metabolites in laying hen droppings-Effects of fiber enrichment, genotype, and daily variations. Poult Sci 93, 2615-2621. https://doi.org/10.3382/ps.2014-04193

Altizer, S., Nunn, C.L., Thrall, P.H., Gittleman, J.L., Antonovics, J., Cunningham, A.A., Dobson, A.P., Ezenwa, V., Jones, K.E., Pedersen, A.B., Poss, M., Pulliam, J.R.C., 2003. Social organization and parasite risk in mammals: Integrating theory and empirical studies. Annu Rev Ecol Evol S 34, 517-547. https://doi.org/10.1146/annurev.ecolsys.34.030102.151725

Altmann, J., Gesquiere, L., Galbany, J., Onyango, P.O., Alberts, S.C., 2010. Life history context of reproductive aging in a wild primate model. Ann N Y Acad Sci 1204, 127-138. https://doi.org/10.1111/j.1749-6632.2010.05531.x

Altschul, S.F., Gish, W., Miller, W., Myers, E.W., Lipman, D.J., 1990. Basic local alignment search tool. J. Mol. Biol. 215, $403-410$. https://doi.org/10.1016/S0022-2836(05)80360-2

Amato, K.R., 2016. Incorporating the gut microbiota into models of human and non-human primate ecology and evolution. Am. J. Phys. Anthropol. 159, 196-215. https://doi.org/10.1002/ajpa.22908

Amato, K.R., Belle, S.V., Fiore, A.D., Estrada, A., Stumpf, R., White, B., Nelson, K.E., Knight, R., Leigh, S.R., 2017a. Patterns in gut microbiota similarity associated with degree of sociality among sex classes of a Neotropical primate. Microb Ecol 74, 250-258. https://doi.org/10.1007/s00248-017-0938-6

Amato, K.R., Leigh, S.R., Kent, A., Mackie, R.I., Yeoman, C.J., Stumpf, R.M., Wilson, B.A., Nelson, K.E., White, B.A., Garber, P.A., 2014. The role of gut microbes in satisfying the nutritional demands of adult and juvenile wild, black howler monkeys (Alouatta pigra). Am. J. Primatol. 155, 652-664. https://doi.org/10.1002/ajpa.22621

Amato, K.R., Martinez-Mota, R., Righini, N., Raguet-Schofield, M., Corcione, F.P., Marini, E., Humphrey, G., Gogul, G., Gaffney, J., Lovelace, E., Williams, L., Luong, A., Dominguez-Bello, M.G., Stumpf, R.M., White, B., Nelson, K.E., Knight, R., Leigh, S.R., 2015. Phylogenetic and ecological factors impact the gut microbiota of two Neotropical primate species. Oecologia 180, 717-733. https://doi.org/10.1007/s00442-015-3507-z

Amato, K.R., Sanders, J.G., Song, S.J., Nute, M., Metcalf, J.L., Thompson, L.R., Morton, J.T., Amir, A., McKenzie, V.J., Humphrey, G., Gogul, G., Gaffney, J., Baden, A.L., Britton, G.A.O., Cuozzo, F.P., Fiore, A.D., Dominy, N.J., Goldberg, T.L., Gomez, A., Kowalewski, M.M., Lewis, R.J., Link, A., Sauther, M.L., Tecot, S., White, B.A., Nelson, K.E., Stumpf, R.M., Knight, R., Leigh, S.R., 2019. Evolutionary trends in host physiology outweigh dietary niche in structuring primate gut microbiomes. ISME J 13, 576-587. https://doi.org/10.1038/s41396-018-0175-0

Amato, K.R., Van Belle, S., Di Fiore, A., Estrada, A., Stumpf, R., White, B., Nelson, K.E., Knight, R., Leigh, S.R., 2017b. Patterns in gut microbiota similarity associated with degree of sociality among sex classes of a neotropical primate. Microb Ecol 74, 250-258. https://doi.org/10.1007/s00248-017-0938-6

Amato, K.R., Yeoman, C.J., Kent, A., Righini, N., Carbonero, F., Estrada, A., Gaskins, H.R., Stumpf, R.M., Yildirim, S., Torralba, M., Gillis, M., Wilson, B.A., Nelson, K.E., White, B.A., Leigh, S.R., 2013. Habitat degradation impacts black howler monkey (Alouatta pigra) gastrointestinal microbiomes. ISME J 7, 1344-1353. https://doi.org/10.1038/ismej.2013.16

Amrein, M., Heistermann, M., Weingrill, T., 2014. The effect of fission-fusion Zoo housing on hormonal and behavioral indicators of stress in Bornean orangutans (Pongo pygmaeus). Int J Primatol 35, 509-528. https://doi.org/10.1007/s10764-014-9765-5

Archie, E.A., 2013. Wound healing in the wild: Stress, sociality and energetic costs affect wound healing in natural populations. Parasite Immunol. 35, 374-385. https://doi.org/10.1111/pim.12048

Archie, E.A., Altmann, J., Alberts, S.C., 2012. Social status predicts wound healing in wild baboons. PNAS 109, 9017-9022. https://doi.org/10.1073/pnas.1206391109 
Archie, E.A., Theis, K.R., 2011. Animal behaviour meets microbial ecology. Animal Behaviour 82, 425-436. https://doi.org/10.1016/j.anbehav.2011.05.029

Archie, E.A., Tung, J., Clark, M., Altmann, J., Alberts, S.C., 2014. Social affiliation matters: Both same-sex and opposite-sex relationships predict survival in wild female baboons. Proc R Soc Lond [Biol] 281, 20141261. https://doi.org/10.1098/rspb.2014.1261

Aureli, F., 1997. Post-conflict anxiety in nonhuman primates: The mediating role of emotion in conflict resolution. Aggr. Behav. 23, 315-328. https://doi.org/10.1002/(SICI)1098-2337(1997)23:5<315::AID-AB2>3.0.CO;2-H

Avilés, L., Tufiño, P., 1998. Colony size and individual fitness in the social spider Anelosimus eximius. Am. Nat. 152, $403-418$. https://doi.org/10.1086/286178

Avramenko, R.W., Redman, E.M., Lewis, R., Yazwinski, T.A., Wasmuth, J.D., Gilleard, J.S., 2015. Exploring the gastrointestinal "Nemabiome": Deep amplicon sequencing to quantify the species composition of parasitic nematode communities. PLoS ONE 10, e0143559. https://doi.org/10.1371/journal.pone.0143559

Baayen, R.H., Davidson, D.J., Bates, D.M., 2008. Mixed-effects modeling with crossed random effects for subjects and items. J. Mem. Lang., Special Issue: Emerging Data Analysis 59, 390-412. https://doi.org/10.1016/j.jml.2007.12.005

Bahr, N.I., Palme, R., Möhle, U., Hodges, J.K., Heistermann, M., 2000. Comparative aspects of the metabolism and excretion of cortisol in three individual nonhuman primates. Gen Comp Endocrinol 117, 427-438. https://doi.org/10.1006/gcen.1999.7431

Bailey, M.T., Dowd, S.E., Galley, J.D., Hufnagle, A.R., Allen, R.G., Lyte, M., 2011. Exposure to a social stressor alters the structure of the intestinal microbiota: Implications for stressor-induced immunomodulation. Brain Behav Immun Health 25, 397-407. https://doi.org/10.1016/j.bbi.2010.10.023

Bales, K.L., French, J.A., McWilliams, J., Lake, R.A., Dietz, J.M., 2006. Effects of social status, age, and season on androgen and cortisol levels in wild male golden lion tamarins (Leontopithecus rosalia). Horm Behav. 49, 88-95. https://doi.org/10.1016/j.yhbeh.2005.05.006

Barelli, C., Albanese, D., Donati, C., Pindo, M., Dallago, C., Rovero, F., Cavalieri, D., Tuohy, K.M., Hauffe, H.C., De Filippo, C., 2015. Habitat fragmentation is associated to gut microbiota diversity of an endangered primate: implications for conservation. Sci Rep 5, 14862. https://doi.org/10.1038/srep14862

Barr, D.J., Levy, R., Scheepers, C., Tily, H.J., 2013. Random effects structure for confirmatory hypothesis testing: Keep it maximal. J Mem Lang 68, 255-278. https://doi.org/10.1016/j.jml.2012.11.001

Barrett, G.M., Shimizu, K., Bardi, M., Asaba, S., Mori, A., 2002. Endocrine correlates of rank, reproduction, and female-directed aggression in male Japanese macaques (Macaca fuscata). Horm Behav 42, 85-96. https://doi.org/10.1006/hbeh.2002.1804

Barros, M., Tomaz, C., 2002. Non-human primate models for investigating fear and anxiety. Neurosci Biobehav Rev 26, $187-$ 201. https://doi.org/10.1016/S0149-7634(01)00064-1

Barton, K., 2018. MuMIn : Multi-Model Inference, R package version 1.42.1.

Barton, R.A., 1987. Allogrooming as mutualism in diurnal lemurs. Primates 28, 539-542. https://doi.org/10.1007/BF02380868

Bates, D., Maechler, M., Bolker, B., Walker, S., 2015. Fitting linear mixed-effects models using Ime4. J. Stat. Softw. 67, 1-48.

Bates, D.M., Maechler, M., Bolker, B.M., 2012. Ime4: Linear mixed-effects models using S4 classes. R Foundation for Statistical Computing, Vienna, Austria. https://CRAN.R-project.org/package=Ime4

Beauchamp, G., 2017. What can vigilance tell us about fear? ASent 53.

Beehner, J.C., Bergman, T.J., 2017. The next step for stress research in primates: To identify relationships between glucocorticoid secretion and fitness. Horm Behav 91, 68-83. https://doi.org/10.1016/j.yhbeh.2017.03.003

Beehner, J.C., Bergman, T.J., Cheney, D.L., Seyfarth, R.M., Whitten, P.L., 2006. Testosterone predicts future dominance rank and mating activity among male chacma baboons. Behav Ecol Sociobiol 59, 469-479. https://doi.org/10.1007/s00265-005-0071-2

Behie, A.M., Pavelka, M.S.M., 2013. Interacting roles of diet, cortisol levels, and parasites in determining population density of Belizean howler monkeys in a hurricane damaged forest fragment, in: Marsh, L.K., Chapman, C.A. (Eds.), Primates in Fragments: Complexity and Resilience, Developments in Primatology: Progress and Prospects. Springer New York, New York, NY, pp. 447-456. https://doi.org/10.1007/978-1-4614-8839-2_30

Behringer, V., Deschner, T., 2017. Non-invasive monitoring of physiological markers in primates. Horm Behav, Field endocrinology of nonhuman primates and humans 91, 3-18. https://doi.org/10.1016/j.yhbeh.2017.02.001

Bekoff, M., Daniels, T.J., Gittleman, J.L., 1984. Life History Patterns and the Comparative Social Ecology of Carnivores. Annu. Rev. Ecol. Evol. Syst 15, 191-232. https://doi.org/10.1146/annurev.es.15.110184.001203

Benadi, G., Fichtel, C., Kappeler, P., 2008. Intergroup relations and home range use in Verreaux's sifaka (Propithecus verreauxi). Am. J. Primatol. 70, 956-965. https://doi.org/10.1002/ajp.20588

Benítez, M.E., Sosnowski, M.J., Tomeo, O.B., Brosnan, S.F., 2018. Urinary oxytocin in capuchin monkeys: Validation and the influence of social behavior. Am. J. Primatol. 80, e22877. https://doi.org/10.1002/ajp.22877

Bennett, G., Malone, M., Sauther, M.L., Cuozzo, F.P., White, B., Nelson, K.E., Stumpf, R.M., Knight, R., Leigh, S.R., Amato, K.R., 2016a. Host age, social group, and habitat type influence the gut microbiota of wild ring-tailed lemurs (Lemur catta). Am. J. Primatol. 78, 883-892. https://doi.org/10.1002/ajp.22555

Bercovitch, F.B., 1997. Reproductive strategies of rhesus macaques. Primates 38, 247-263. https://doi.org/10.1007/BF02381613

Berdoy, M., Smith, P., MacDonald, D.W., 1995. Stability of social status in wild rats: Age and the role of settled dominance. Behaviour 132, 193-212. 
Bergman, T.J., Beehner, J.C., Cheney, D.L., Seyfarth, R.M., Whitten, P.L., 2005. Correlates of stress in free-ranging male chacma baboons, Papio hamadryas ursinus. Anim Behav 70, 703-713. https://doi.org/10.1016/j.anbehav.2004.12.017

Bernard, G., Pathmanathan, J.S., Lannes, R., Lopez, P., Bapteste, E., 2018. Microbial dark matter investigations: How microbial studies transform biological knowledge and empirically sketch a logic of scientific discovery. Genome Biol Evol 10, 707-715. https://doi.org/10.1093/gbe/evy031

Besedovsky, H., Rey, A. del, Sorkin, E., Dinarello, C.A., 1986. Immunoregulatory feedback between interleukin-1 and glucocorticoid hormones. Science 233, 652-654. https://doi.org/10.1126/science.3014662

Bilde, T., Coates, K.S., Birkhofer, K., Bird, T., Maklakov, A.A., Lubin, Y., Avilés, L., 2007. Survival benefits select for group living in a social spider despite reproductive costs. J Evol Biol 20, 2412-2426. https://doi.org/10.1111/j.14209101.2007.01407.x

Björk, J.R., Dasari, M., Grieneisen, L., Archie, E.A., 2019. Primate microbiomes over time: Longitudinal answers to standing questions in microbiome research. Am J Primatol, e22970. https://doi.org/10.1002/ajp.22970

Boccia, M.L., Reite, M., Laudenslager, M., 1989. On the physiology of grooming in a pigtail macaque. Physiology \& Behavior 45, 667-670. https://doi.org/10.1016/0031-9384(89)90089-9

Boerner, B.P., Sarvetnick, N.E., 2011. Type 1 diabetes: role of intestinal microbiome in humans and mice. Ann N Y Acad Sci 1243, 103-118. https://doi.org/10.1111/j.1749-6632.2011.06340.x

Boesch, C., Kohou, G., Néné, H., Vigilant, L., 2006. Male competition and paternity in wild chimpanzees of the Taï forest. Am J Phys Anthropol 130, 103-115. https://doi.org/10.1002/ajpa.20341

Boinski, S., 1987. Mating patterns in squirrel monkeys (Saimiri oerstedi). Behav Ecol Sociobiol 21, 13-21. https://doi.org/10.1007/BF00324430

Bókony, V., Lendvai, Á.Z., Liker, A., Angelier, F., Wingfield, J.C., Chastel, O., 2009. Stress response and the value of reproduction: Are birds prudent parents? Am Nat 173, 589-598. https://doi.org/10.1086/597610

Bokulich, N.A., Chung, J., Battaglia, T., Henderson, N., Jay, M., Li, H., Lieber, A.D., Wu, F., Perez-Perez, G.I., Chen, Y., Schweizer, W., Zheng, X., Contreras, M., Dominguez-Bello, M.G., Blaser, M.J., 2016. Antibiotics, birth mode, and diet shape microbiome maturation during early life. Sci Transl Med 8, 343ra82-343ra82. https://doi.org/10.1126/scitranslmed.aad7121

Bolker, B.M., Brooks, M.E., Clark, C.J., Geange, S.W., Poulsen, J.R., Stevens, M.H.H., White, J.-S.S., 2009. Generalized linear mixed models: a practical guide for ecology and evolution. Trends Ecol Evol 24, 127-135. https://doi.org/10.1016/j.tree.2008.10.008

Boonstra, R., Boag, P.T., 1992. Spring declines in Microtus pennsylvanicus and the role of steroid hormones. J Anim Ecol 61 , 339-352. https://doi.org/10.2307/5326

Boutin, S., Bernatchez, L., Audet, C., Derôme, N., 2013. Network analysis highlights complex linteractions between pathogen, host and commensal microbiota. PLOS ONE 8, e84772. https://doi.org/10.1371/journal.pone.0084772

Braga Goncalves, I., Heistermann, M., Santema, P., Dantzer, B., Mausbach, J., Ganswindt, A., Manser, M.B., 2016. Validation of a fecal glucocorticoid assay to assess adrenocortical activity in meerkats using physiological and biological stimuli. PLOS ONE 11, e0153161. https://doi.org/10.1371/journal.pone.0153161

Brito, I.L., Gurry, T., Zhao, S., Huang, K., Young, S.K., Shea, T.P., Naisilisili, W., Jenkins, A.P., Jupiter, S.D., Gevers, D., Alm, E.J., 2019. Transmission of human-associated microbiota along family and social networks. Nat Microbiol 4, 964-971. https://doi.org/10.1038/s41564-019-0409-6

Brockman, D.K., 1999. Reproductive behavior of female Propithecus verreauxi at Beza Mahafaly, Madagascar. Int J Primatol 20, 375-398. https://doi.org/10.1023/A:1020500804442

Brockman, D.K., Cobden, A.K., Whitten, P.L., 2009. Birth season glucocorticoids are related to the presence of infants in sifaka (Propithecus verreauxi). Proc. R. Soc. B 276, 1855-1863. https://doi.org/10.1098/rspb.2008.1912

Brockman, D.K., Whitten, P.L., Richard, A.F., Schneider, A., 1998. Reproduction in free-ranging male Propithecus verreauxi: The hormonal correlates of mating and aggression. Am J Phys Anthropol 105, 137-151. https://doi.org/10.1002/(SICI)1096-8644(199802)105:2<137::AID-AJPA3>3.0.CO;2-S

Bronikowski, A.M., Altmann, J., 1996. Foraging in a variable environment: Weather patterns and the behavioral ecology of baboons. Behav Ecol Sociobiol 39, 11-25. https://doi.org/10.1007/s002650050262

Brooks, M.E., Kristensen, K., van Benthem, K.J., Magnusson, A., Berg, C.W., Nielsen, A., Skaug, H.J., Machler, M., Bolker, B.M., 2017. glmmTMB balances speed and flexibility among packages for zero-inflated generalized linear mixed modeling. The R journal 9, 378-400. https://doi.org/10.3929/ethz-b-000240890

Brown, J.L., 1975. The Evolution of Behaviour. New York (Norto) 1975.

Browne, H.P., Neville, B.A., Forster, S.C., Lawley, T.D., 2017. Transmission of the gut microbiota: spreading of health. Nat Rev Micro 15, 531-543. https://doi.org/10.1038/nrmicro.2017.50

Busch, D.S., Hayward, L.S., 2009. Stress in a conservation context: A discussion of glucocorticoid actions and how levels change with conservation-relevant variables. Biol Conserv 142, 2844-2853. https://doi.org/10.1016/j.biocon.2009.08.013

Bush, A.O., Fernández, J.C., Esch, G.W., Seed, J.R., 2001. Parasitism: The diversity and ecology of animal parasites. Cambridge University Press.

Calenge, C., 2006. The package "adehabitat" for the R software: A tool for the analysis of space and habitat use by animals. Ecol Modell 197, 516-519. https://doi.org/10.1016/j.ecolmodel.2006.03.017

Cameron, E.Z., Setsaas, T.H., Linklater, W.L., 2009. Social bonds between unrelated females increase reproductive success in feral horses. PNAS 106, 13850-13853. https://doi.org/10.1073/pnas.0900639106 
Caporaso, J.G., Kuczynski, J., Stombaugh, J., Bittinger, K., Bushman, F.D., Costello, E.K., Fierer, N., Peña, A.G., Goodrich, J.K., Gordon, J.I., Huttley, G.A., Kelley, S.T., Knights, D., Koenig, J.E., Ley, R.E., Lozupone, C.A., McDonald, D., Muegge, B.D., Pirrung, M., Reeder, J., Sevinsky, J.R., Turnbaugh, P.J., Walters, W.A., Widmann, J., Yatsunenko, T., Zaneveld, J., Knight, R., 2010. QIIME allows analysis of high-throughput community sequencing data. Nat Methods 7, 335-336. https://doi.org/10.1038/nmeth.f.303

Carabotti, M., Scirocco, A., Maselli, M.A., Severi, C., 2015. The gut-brain axis: Interactions between enteric microbiota, central and enteric nervous systems. Ann Gastroenterol 28, 203-209.

Caraco, T., 1979. Time budgeting and group size: A theory. Ecology 60, 611-617. https://doi.org/10.2307/1936081

Carrai, V., Borgognini-Tarli, S.M., Huffman, M.A., Bardi, M., 2003. Increase in tannin consumption by sifaka (Propithecus verreauxi verreauxi) females during the birth season: a case for self-medication in prosimians? Primates 44, 61-66. https://doi.org/10.1007/s10329-002-0008-6

Castracane, V.D., Cutler, G.B., Loriaux, D.L., 1981. Pubertal endocrinology of the baboon: Adrenarche. Am J Physiol Endocrinol Metab 241, E305-E309. https://doi.org/10.1152/ajpendo.1981.241.4.E305

Cavigelli, S.A., Caruso, M.J., 2015. Sex, social status and physiological stress in primates: the importance of social and glucocorticoid dynamics. Proc. R. Soc. B 370, 20140103. https://doi.org/10.1098/rstb.2014.0103

Cavigelli, S.A., Dubovick, T., Levash, W., Jolly, A., Pitts, A., 2003. Female dominance status and fecal corticoids in a cooperative breeder with low reproductive skew: ring-tailed lemurs (Lemur catta). Horm Behav 43, 166-179. https://doi.org/10.1016/S0018-506X(02)00031-4

Chapman, C.A., Chapman, L.J., 2000. Determinants of group size in primates: the importance of travel costs., in: Boinski, S., Garber, P.A. (Eds.), On the Move: How and Why Animals Travel in Groups. University of Chicago Press, Chicago, pp. 24-42.

Chapman, C.A., Gillespie, T.R., Speirs, M.L., 2005. Parasite prevalence and richness in sympatric colobines: effects of host density. Am. J. Primatol. 67, 259-266. https://doi.org/10.1002/ajp.20181

Chapman, C.A., Saj, T.L., Snaith, T.V., 2007. Temporal dynamics of nutrition, parasitism, and stress in colobus monkeys: Implications for population regulation and conservation. Am. J. Phys. Anthropol. 134, 240-250. https://doi.org/10.1002/ajpa.20664

Charpentier, M.J.E., Givalois, L., Faurie, C., Soghessa, O., Simon, F., Kappeler, P.M., 2018. Seasonal glucocorticoid production correlates with a suite of small-magnitude environmental, demographic, and physiological effects in mandrills. Am J Phys Anthropol 165, 20-33. https://doi.org/10.1002/ajpa.23329

Chatfield, K., 2018. Defining health, in: Chatfield, K. (Ed.), Traditional and Complementary Medicines: Are They Ethical for Humans, Animals and the Environment?, SpringerBriefs in Philosophy. Springer International Publishing, Cham, pp. 17-28. https://doi.org/10.1007/978-3-030-05300-0_2

Chen, J., Bittinger, K., Charlson, E.S., Hoffmann, C., Lewis, J., Wu, G.D., Collman, R.G., Bushman, F.D., Li, H., 2012. Associating microbiome composition with environmental covariates using generalized UniFrac distances. Bioinformatics 28 , 2106-2113. https://doi.org/10.1093/bioinformatics/bts342

Chen, L., Reeve, J., Zhang, L., Huang, S., Wang, X., Chen, J., 2018. GMPR: A robust normalization method for zero-inflated count data with application to microbiome sequencing data. PeerJ 6, e4600. https://doi.org/10.7717/peerj.4600

Chen, S., Zhou, Y., Chen, Y., Gu, J., 2018. fastp: An ultra-fast all-in-one FASTQ preprocessor. Bioinformatics 34, i884-i890. https://doi.org/10.1093/bioinformatics/bty560

Chiyo, P.I., Grieneisen, L.E., Wittemyer, G., Moss, C.J., Lee, P.C., Douglas-Hamilton, I., Archie, E.A., 2014. The influence of social structure, habitat, and host traits on the transmission of Escherichia coli in wild elephants. PLOS ONE 9, e93408. https://doi.org/10.1371/journal.pone.0093408

Chmura, H.E., Wey, T.W., Blumstein, D.T., 2016. Assessing the sensitivity of foraging and vigilance to internal state and environmental variables in yellow-bellied marmots (Marmota flaviventris). Behav Ecol Sociobiol 70, 1901-1910. https://doi.org/10.1007/s00265-016-2195-y

Chow, J., Lee, S.M., Shen, Y., Khosravi, A., Mazmanian, S.K., 2010. Chapter 8 - Host-Bacterial Symbiosis in Health and Disease, in: Fagarasan, S., Cerutti, A. (Eds.), Advances in Immunology, Mucosal Immunity. Academic Press, pp. 243-274. https://doi.org/10.1016/B978-0-12-381300-8.00008-3

Clary, D., Skyner, L.J., Ryan, C.P., Gardiner, L.E., Anderson, W.G., Hare, J.F., 2014. Shyness-boldness, but not exploration, predicts glucocorticoid stress response in Richardson's Ground squirrels (Urocitellus richardsonii). Ethology 120, 1101-1109. https://doi.org/10.1111/eth.12283

Clayton, J.B., Gomez, A., Amato, K., Knights, D., Travis, D.A., Blekhman, R., Knight, R., Leigh, S., Stumpf, R., Wolf, T., Glander, K.E., Cabana, F., Johnson, T.J., 2018. The gut microbiome of nonhuman primates: Lessons in ecology and evolution. Am J Primatol 0, e22867. https://doi.org/10.1002/ajp.22867

Clinchy, M., Sheriff, M.J., Zanette, L.Y., 2013. Predator-induced stress and the ecology of fear. Funct. Ecol. 27, 56-65. https://doi.org/10.1111/1365-2435.12007

Clough, D., Heistermann, M., Kappeler, P.M., 2010. Host intrinsic determinants and potential consequences of parasite infection in free-ranging red-fronted lemurs (Eulemur fulvus rufus). Am. J. Phys. Anthropol. 142, 441-452. https://doi.org/10.1002/ajpa.21243

Clutton-Brock, T.H., Harvey, P.H., 1977. Primate ecology and social organization. J Zool 183, 1-39. https://doi.org/10.1111/j.1469-7998.1977.tb04171.x

Cohen, S., 2004. Social relationships and health. Am Psychol 59, 676-684.

Cohen, S., Doyle, W.J., Skoner, D.P., Rabin, B.S., Gwaltney, J.M., 1997. Social ties and susceptibility to the common cold. JAMA 277, 1940-1944. https://doi.org/10.1001/jama.1997.03540480040036 
Cohen, S., Janicki-Deverts, D., Doyle, W.J., Miller, G.E., Frank, E., Rabin, B.S., Turner, R.B., 2012. Chronic stress, glucocorticoid receptor resistance, inflammation, and disease risk. PNAS 109, 5995-5999. https://doi.org/10.1073/pnas.1118355109

Collado, M.C., Rautava, S., Aakko, J., Isolauri, E., Salminen, S., 2016. Human gut colonisation may be initiated in utero by distinct microbial communities in the placenta and amniotic fluid. Sci Rep 6, 1-13. https://doi.org/10.1038/srep23129

Cords, M., 1995. Predator Vigilance Costs of allogrooming in wild Blue monkeys. Behaviour 132, 559-569. https://doi.org/10.1163/156853995X00207

Costa, J.T., Fitzgerald, T.D., 2005. Social terminology revisited: Where are we ten years later? Ann Zool Fenn 42, 559-564.

Costa, J.T., Fitzgerald, T.D., 1996. Developments in social terminology: semantic battles in a conceptual war. Trends Ecol Evol 11, 285-289. https://doi.org/10.1016/0169-5347(96)10035-5

Creel, S., 2001. Social dominance and stress hormones. Trends Ecol Evol 16, 491-497. https://doi.org/10.1016/S01695347(01)02227-3

Creel, S., Dantzer, B., Goymann, W., Rubenstein, D.R., 2013. The ecology of stress: Effects of the social environment. Funct Ecol 27, 66-80. https://doi.org/10.1111/j.1365-2435.2012.02029.x

Cremer, S., Armitage, S.A.O., Schmid-Hempel, P., 2007. Social immunity. Current Biology 17, R693-R702. https://doi.org/10.1016/j.cub.2007.06.008

Cresci, G.A., Bawden, E., 2015. Gut microbiome: What we do and don't know. Nutr Clin Pract 30, 734-746. https://doi.org/10.1177/0884533615609899

Crockford, C., Wittig, R.M., Whitten, P.L., Seyfarth, R.M., Cheney, D.L., 2008. Social stressors and coping mechanisms in wild female baboons (Papio hamadryas ursinus). Horm Behavi 53, 254-265. https://doi.org/10.1016/j.yhbeh.2007.10.007

Crook, J.H., Gartlan, J.S., 1966. Evolution of primate societies. Nature 210, 1200-1203.

Csárdi, G., Nepusz, T., 2006. The igraph software package for complex network research. InterJournal Complex Systems 1695, 1-9. http://igraph.org

Culbert, B.M., Gilmour, K.M., Balshine, S., 2018. Stress axis regulation during social ascension in a group-living cichlid fish. Horm Behav 103, 121-128. https://doi.org/10.1016/j.yhbeh.2018.06.007

Dąbrowska, K., Witkiewicz, W., 2016. Correlations of host genetics and gut microbiome composition Front. Microbiol. 7. https://doi.org/10.3389/fmicb.2016.01357

Dantzer, B., Bennett, N.C., Clutton-Brock, T.H., 2017. Social conflict and costs of cooperation in meerkats are reflected in measures of stress hormones. Behav Ecol 28, 1131-1141. https://doi.org/10.1093/beheco/arx077

Dantzer, B., McAdam, A.G., Palme, R., Boutin, S., Boonstra, R., 2011. How does diet affect fecal steroid hormone metabolite concentrations? An experimental examination in red squirrels. Gen Comp Endocrinol 174, 124-131. https://doi.org/10.1016/j.ygcen.2011.08.010

Dantzer, B., Newman, A.E.M., Boonstra, R., Palme, R., Boutin, S., Humphries, M.M., McAdam, A.G., 2013. Density triggers maternal hormones that increase adaptive offspring growth in a wild mammal. Science 340, 1215-1217. https://doi.org/10.1126/science.1235765

Dausmann, K.H., 2014. Flexible patterns in energy savings: heterothermy in primates. Journal of Zoology 292, 101-111. https://doi.org/10.1111/jzo.12104

David, L.A., Maurice, C.F., Carmody, R.N., Gootenberg, D.B., Button, J.E., Wolfe, B.E., Ling, A.V., Devlin, A.S., Varma, Y., Fischbach, M.A., Biddinger, S.B., Dutton, R.J., Turnbaugh, P.J., 2014. Diet rapidly and reproducibly alters the human gut microbiome. Nature 505, 559-563. https://doi.org/10.1038/nature12820

Defolie, C., Merkling, T., Fichtel, C., 2019. Patterns and variation in the mammal parasite-glucocorticoid relationship. Biol Rev https://doi.org/10.1111/brv.12555

Degnan, P.H., Pusey, A.E., Lonsdorf, E.V., Goodall, J., Wroblewski, E.E., Wilson, M.L., Rudicell, R.S., Hahn, B.H., Ochman, H., 2012a. Factors associated with the diversification of the gut microbial communities within chimpanzees from Gombe National Park. PNAS 109, 13034-13039. https://doi.org/10.1073/pnas.1110994109

Degnan, P.H., Pusey, A.E., Lonsdorf, E.V., Goodall, J., Wroblewski, E.E., Wilson, M.L., Rudicell, R.S., Hahn, B.H., Ochman, H., $2012 \mathrm{~b}$. Factors associated with the diversification of the gut microbial communities within chimpanzees from Gombe National Park. PNAS 109, 13034-13039. https://doi.org/10.1073/pnas.1110994109

DeVore, I., 1963. A comparison of the ecology and behavior of monkeys and apes, in: Classification and Human Evolution. Aldine Publishing Company, Chicago.

Dewar, R.E., Richard, A.F., 2007. Evolution in the hypervariable environment of Madagascar. PNAS 104, 13723-13727. https://doi.org/10.1073/pnas.0704346104

Dhabhar, F.S., 2009. Enhancing versus suppressive effects of stress on immune function: Implications for immunoprotection and immunopathology. NIM 16, 300-317. https://doi.org/10.1159/000216188

Dhabhar, F.S., Miller, A.H., McEwen, B.S., Spencer, R.L., 1996. Stress-induced changes in blood leukocyte distribution. Role of adrenal steroid hormones. J Immunol 157, 1638-1644.

Dobson, A.J., 2002. An introduction to generalized linear mixed models, 2nd ed. Chapman \& Hall, New York, NY.

Dominianni, C., Sinha, R., Goedert, J.J., Pei, Z., Yang, L., Hayes, R.B., Ahn, J., 2015. Sex, body mass index, and dietary fiber intake influence the human gut microbiome. PLOS ONE 10, e0124599. https://doi.org/10.1371/journal.pone.0124599

Dreiss, A.N., Ruppli, C.A., Oberli, F., Antoniazza, S., Henry, I., Roulin, A., 2013. Barn owls do not interrupt their siblings. Anim Behav 86, 119-126. https://doi.org/10.1016/j.anbehav.2013.04.019

Drews, C., 1993. The concept and definition of dominance in animal behaviour. Behaviour 125, 283-313. https://doi.org/10.1163/156853993X00290 
Duboscq, J., Romano, V., Sueur, C., MacIntosh, A.J.J., 2016. Network centrality and seasonality interact to predict lice load in a social primate. Sci Rep 6, 1-13. https://doi.org/10.1038/srep22095

Dunbar, R.I.M., 1991. Functional significance of social grooming in primates. FPR 57, 121-131. https://doi.org/10.1159/000156574

Dunbar, R.I.M., Korstjens, A.H., Lehmann, J., 2009. Time as an ecological constraint. Biol Rev 84, 413-429. https://doi.org/10.1111/j.1469-185X.2009.00080.x

Dunn, J.C., Cristóbal-Azkarate, J., Schulte-Herbrüggen, B., Chavira, R., Veà, J.J., 2013. Travel time predicts fecal glucocorticoid levels in free-ranging howlers (Alouatta palliata). Int J Primatol 34, 246-259. https://doi.org/10.1007/s10764-0139657-0

Eaglen, R.H., 1980. Toothcomb homology and toothcomb function in extant strepsirhines. Int J Primatol 1, 275-286. https://doi.org/10.1007/BF02692275

Eberle, M., Kappeler, P.M., 2004. Selected polyandry: female choice and inter-sexual conflict in a small nocturnal solitary primate (Microcebus murinus). Behav Ecol Sociobiol 57, 91-100. https://doi.org/10.1007/s00265-004-0823-4

Edgar, R.C., 2010. Search and clustering orders of magnitude faster than BLAST. Bioinformatics 26, 2460-2461. https://doi.org/10.1093/bioinformatics/btq461

Elenkov, I.J., Chrousos, G.P., 1999. Stress hormones, Th1/Th2 patterns, pro/anti-inflammatory cytokines and susceptibility to disease. Trends Endocrinol Metab 10, 359-368. https://doi.org/10.1016/S1043-2760(99)00188-5

Ellis, J.J., MacLarnon, A.M., Heistermann, M., Semple, S., 2011. The social correlates of self-directed behaviour and faecal glucocorticoid levels among adult male olive baboons (Papio hamadryas anubis) in Gashaka-Gumti National Park, Nigeria. African Zoology 46, 302-308. https://doi.org/10.1080/15627020.2011.11407503

Emery Thompson, M., 2017. Energetics of feeding, social behavior, and life history in non-human primates. Horm Behav, Field endocrinology of nonhuman primates and humans 91, 84-96. https://doi.org/10.1016/j.yhbeh.2016.08.009

Emery Thompson, M., Muller, M.N., Kahlenberg, S.M., Wrangham, R.W., 2010. Dynamics of social and energetic stress in wild female chimpanzees. Horm Behav 58, 440-449. https://doi.org/10.1016/j.yhbeh.2010.05.009

Emlen, S.T., Oring, L.W., 1977. Ecology, sexual selection, and the evolution of mating systems. Science 197, $215-223$.

Eppley, T.M., Watzek, J., Dausmann, K.H., Ganzhorn, J.U., Donati, G., 2017a. Huddling is more important than rest site selection for thermoregulation in southern bamboo lemurs. Anim Behav 127, 153-161. https://doi.org/10.1016/j.anbehav.2017.03.019

Eppley, T.M., Watzek, J., Hall, K., Donati, G., 2017b. Climatic, social and reproductive influences on behavioural thermoregulation in a female-dominated lemur. Anim Behav 134, 25-34. https://doi.org/10.1016/j.anbehav.2017.10.003

Erhart, E.M., Overdorff, D.J., 2008. Rates of agonism by diurnal lemuroids: Implications for female social relationships. Int J Primatol 29, 1227-1247. https://doi.org/10.1007/s10764-008-9287-0

Erhart, E.M., Overdorff, D.J., 1998. Infanticide in Propithecus diadema edwardsi: An evaluation of the sexual selection hypothesis. Int J Primatol 19, 73-81. https://doi.org/10.1023/A:1020306910493

Erkert, H.G., Kappeler, P.M., 2004. Arrived in the light: diel and seasonal activity patterns in wild Verreaux's sifakas (Propithecus v. verreauxi; Primates: Indriidae). Behav Ecol Sociobiol 57, 174-186. https://doi.org/10.1007/s00265-004-0845-y

Ezenwa, V.O., 2004. Host social behavior and parasitic infection: a multifactorial approach. Behav Ecol 15, 446-454. https://doi.org/10.1093/beheco/arh028

Ezenwa, V.O., Gerardo, N.M., Inouye, D.W., Medina, M., Xavier, J.B., 2012. Animal behavior and the microbiome. Science 338, 198-199. https://doi.org/10.1126/science.1227412

Ezenwa, V.O., Ghai, R.R., McKay, A.F., Williams, A.E., 2016. Group living and pathogen infection revisited. Curr Opin Behav Sci, Behavioral ecology 12, 66-72. https://doi.org/10.1016/j.cobeha.2016.09.006

Fairbanks, B., Hawley, D.M., 2012. Interactions between host social behavior, physiology, and disease susceptibility: the role of dominance status and social context., in: Ecoimmunology. Oxford University Press, USA, pp. 440-467.

Faith, D.P., 1992. Conservation evaluation and phylogenetic diversity. Biol Conserv 61, 1-10.

Fauci, A.S., Dale, D.C., 1974. The effect of in vivo hydrocortisone on subpopulations of human lymphocytes. J Clin Invest 53, 240-246. https://doi.org/10.1172/JCl107544

Federman, S., Sinnott-Armstrong, M., Baden, A.L., Chapman, C.A., Daly, D.C., Richard, A.R., Valenta, K., Donoghue, M.J., 2017. The Paucity of Frugivores in Madagascar May Not Be Due to Unpredictable Temperatures or Fruit Resources. PLOS ONE 12, e0168943. https://doi.org/10.1371/journal.pone.0168943

Ferguson, D., Signoretto, C., 2011. Environmental persistence and naturalization of fecal indicator organisms, in: Hagedorn, C., Blanch, A.R., Harwood, V.J. (Eds.), Microbial Source Tracking: Methods, Applications, and Case Studies. Springer, New York, NY, pp. 379-397. https://doi.org/10.1007/978-1-4419-9386-1_17

Fichtel, C., Kraus, C., Ganswindt, A., Heistermann, M., 2007. Influence of reproductive season and rank on fecal glucocorticoid levels in free-ranging male Verreaux's sifakas (Propithecus verreauxi). Horm Behav 51, 640-648. https://doi.org/10.1016/j.yhbeh.2007.03.005

Field, A., 2009. Discovering statistics using SPSS, in: An R Companion to Applied Regression. Sage Publications.

Flack, J.C., Waal, F. de, 2007. Context modulates signal meaning in primate communication. PNAS 104, 1581-1586. https://doi.org/10.1073/pnas.0603565104

Fleagle, J.G., 2013. Primate adaptations, in: Primate Adaptation and Evolution: 3rd Edn. Academic Press.

Flint, H.J., Scott, K.P., Duncan, S.H., Louis, P., Forano, E., 2012. Microbial degradation of complex carbohydrates in the gut. Gut Microbes 3, 289-306. https://doi.org/10.4161/gmic.19897 
Foerster, S., Cords, M., Monfort, S.L., 2012. Seasonal energetic stress in a tropical forest primate: Proximate causes and evolutionary implications. PLOS ONE 7, e50108. https://doi.org/10.1371/journal.pone.0050108

Foley, C.A.H., Papageorge, S., Wasser, S.K., 2001. Noninvasive stress and reproductive measures of social and ecological pressures in free-ranging African elephants. Conserv Biol 15, 1134-1142. https://doi.org/10.1046/j.15231739.2001.0150041134.x

Forstmeier, W., Schielzeth, H., 2011. Cryptic multiple hypotheses testing in linear models: overestimated effect sizes and the winner's curse. Behav Ecol Sociobiol 65, 47-55. https://doi.org/10.1007/s00265-010-1038-5

Foster, J.A., Rinaman, L., Cryan, J.F., 2017. Stress \& the gut-brain axis: Regulation by the microbiome. Neurobiology of Stress 7 , 124-136. https://doi.org/10.1016/j.ynstr.2017.03.001

Fournier, L.A., 1974. Un método cuantitativo para la medición de características fenológicas en árboles. Turrialba 24, 422-423.

Fox, J., Weisberg, S., 2011. Multivariate linear models in R. In: An R Companion to Applied Regression, Los Angeles: Thousand Oaks.

Franklin, A.B., Anderson, D.R., Gutiérrez, R.J., Burnham, K.P., 2000. Climate, habitat quality, and fitness in Northern spotted owl populations in Northwestern California. Ecol Monogr 70, 539-590. https://doi.org/10.1890/00129615(2000)070[0539:CHQAFI]2.0.CO;2

Freeland, W.J., 1976. Pathogens and the evolution of primate sociality. Biotropica 8, 12-24. https://doi.org/10.2307/2387816

French, S.S., Matt, K.S., Moore, M.C., 2006. The effects of stress on wound healing in male tree lizards (Urosaurus ornatus). Gen Comp Endocrinol 145, 128-132. https://doi.org/10.1016/j.ygcen.2005.08.005

Frère, C.H., Krützen, M., Mann, J., Connor, R.C., Bejder, L., Sherwin, W.B., 2010. Social and genetic interactions drive fitness variation in a free-living dolphin population. PNAS 107, 19949-19954. https://doi.org/10.1073/pnas.1007997107

Friant, S., Ziegler, T.E., Goldberg, T.L., 2016. Primate reinfection with gastrointestinal parasites: behavioural and physiological predictors of parasite acquisition. Anim Behav 117, 105-113. https://doi.org/10.1016/j.anbehav.2016.04.006

Funkhouser, L.J., Bordenstein, S.R., 2013. Mom knows best: The universality of maternal microbial transmission. PLOS Biology 11, e1001631. https://doi.org/10.1371/journal.pbio.1001631

Fürtbauer, I., Heistermann, M., Schülke, O., Ostner, J., 2014. Low female stress hormone levels are predicted by same- or opposite-sex sociality depending on season in wild Assamese macaques. Psychoneuroendocrinology 48, 19-28. https://doi.org/10.1016/j.psyneuen.2014.05.022

Ganas, J., Robbins, M.M., 2005. Ranging behavior of the mountain gorillas (Gorilla beringei beringei) in Bwindi Impenetrable National Park, Uganda: a test of the ecological constraints model. Behav Ecol Sociobiol 58, 277-288. https://doi.org/10.1007/s00265-005-0920-z

Ganzhorn, J.U., 2002. Distribution of a folivorous lemur in relation to seasonally varying food resources: integrating quantitative and qualitative aspects of food characteristics. Oecologia 131, 427-435. https://doi.org/10.1007/s00442-002-0891-y

Genoud, M., 2002. Comparative studies of basal rate of metabolism in primates. Evol Anthropol 11, 108-111. https://doi.org/10.1002/evan.10070

Gesquiere, L.R., Learn, N.H., Simao, M.C.M., Onyango, P.O., Alberts, S.C., Altmann, J., 2011. Life at the top: Rank and stress in wild male baboons. Science 333, 357-360. https://doi.org/10.1126/science.1207120

Gilbert, J.A., 2015. Social behavior and the microbiome. eLife 4. https://doi.org/10.7554/eLife.07322

Gill, S.R., Pop, M., DeBoy, R.T., Eckburg, P.B., Turnbaugh, P.J., Samuel, B.S., Gordon, J.I., Relman, D.A., Fraser-Liggett, C.M., Nelson, K.E., 2006. Metagenomic analysis of the human distal gut microbiome. Science 312, 1355-1359. https://doi.org/10.1126/science.1124234

Giraldeau, L.-A., Caraco, T., 2018. Social Foraging Theory. Princeton University Press.

Girard-Buttoz, C., Heistermann, M., Krummel, S., Engelhardt, A., 2009. Seasonal and social influences on fecal androgen and glucocorticoid excretion in wild male long-tailed macaques (Macaca fascicularis). Physiology \& Behavior 98, $168-175$. https://doi.org/10.1016/j.physbeh.2009.05.005

Gogarten, J.F., Davies, T.J., Benjamino, J., Gogarten, J.P., Graf, J., Mielke, A., Mundry, R., Nelson, M.C., Wittig, R.M., Leendertz, F.H., Calvignac-Spencer, S., 2018. Factors influencing bacterial microbiome composition in a wild non-human primate community in Taï National Park, Côte d'Ivoire. ISME J. https://doi.org/10.1038/s41396-018-0166-1

Gomez, A., Rothman, J.M., Petrzelkova, K., Yeoman, C.J., Vlckova, K., Umaña, J.D., Carr, M., Modry, D., Todd, A., Torralba, M., Nelson, K.E., Stumpf, R.M., Wilson, B.A., Blekhman, R., White, B.A., Leigh, S.R., 2016. Temporal variation selects for diet-microbe co-metabolic traits in the gut of Gorilla spp. ISME J 10, 514-526. https://doi.org/10.1038/ismej.2015.146

Goodfellow, C.K., Whitney, T., Christie, D.M., Sicotte, P., Wikberg, E.C., Ting, N., 2019. Divergence in gut microbial communities mirrors a social group fission event in a black-and-white colobus monkey (Colobus vellerosus). Am J Primatol 0 , e22966. https://doi.org/10.1002/ajp.22966

Goodrich, J.K., Waters, J.L., Poole, A.C., Sutter, J.L., Koren, O., Blekhman, R., Beaumont, M., Van Treuren, W., Knight, R., Bell, J.T., Spector, T.D., Clark, A.G., Ley, R.E., 2014. Human genetics shape the gut microbiome. Cell 159, 789-799. https://doi.org/10.1016/j.cell.2014.09.053

Gordon, T.P., Rose, R.M., Bernstein, I.S., 1976. Seasonal rhythm in plasma testosterone levels in the rhesus monkey (Macaca mulatta): A three year study. Horm Behav 7, 229-243. https://doi.org/10.1016/0018-506X(76)90050-7

Gould, L., Overdorff, D.J., 2002. Adult male scent-marking in Lemur catta and Eulemur fulvus rufus. Int J Primatol 23, 575-586. https://doi.org/10.1023/A:1014921701106

Gould, L., Ziegler, T.E., Wittwer, D.J., 2005. Effects of reproductive and social variables on fecal glucocorticoid levels in a sample of adult male ring-tailed lemurs (Lemur catta) at the Beza Mahafaly Reserve, Madagascar. Am J Primatol 67, 5-23. https://doi.org/10.1002/ajp.20166 
Goymann, W., 2012. On the use of non-invasive hormone research in uncontrolled, natural environments: The problem with sex, diet, metabolic rate and the individual. Methods Ecol Evol 3, 757-765. https://doi.org/10.1111/j.2041210X.2012.00203.x

Goymann, W., 2005. Noninvasive monitoring of hormones in bird droppings: Physiological validation, sampling, extraction, sex differences, and the influence of diet on hormone metabolite levels. Ann N Y Acad Sci 1046, 35-53. https://doi.org/10.1196/annals.1343.005

Goymann, W., East, M.L., Wachter, B., Höner, O.P., Möstl, E., Van’t Holf, T.J., Hofer, H., 2001. Social, state-dependent and environmental modulation of faecal corticosteroid levels in free-ranging female spotted hyenast. Proc. R. Soc. B 268, 2453-2459. https://doi.org/10.1098/rspb.2001.1828

Goymann, W., Wingfield, J.C., 2004. Allostatic load, social status and stress hormones: the costs of social status matter. Anim Behav 67, 591-602. https://doi.org/10.1016/j.anbehav.2003.08.007

Greene, L.K., Bornbusch, S.L., McKenney, E.A., Harris, R.L., Gorvetzian, S.R., Yoder, A.D., Drea, C.M., 2019a. The importance of scale in comparative microbiome research: New insights from the gut and glands of captive and wild lemurs. Am J Primatol, e22974. https://doi.org/10.1002/ajp.22974

Greene, L.K., Clayton, J.B., Rothman Ryan S., Semel Brandon P., Semel Meredith A., Gillespie Thomas R., Wright Patricia C., Drea Christine M., 2019b. Local habitat, not phylogenetic relatedness, predicts gut microbiota better within folivorous than frugivorous lemur lineages. Biol Lett 15, 20190028. https://doi.org/10.1098/rsbl.2019.0028

Greene, L.K., McKenney, E.A., O'Connell, T.M., Drea, C.M., 2018. The critical role of dietary foliage in maintaining the gut microbiome and metabolome of folivorous sifakas. Sci Rep 8, 1-13. https://doi.org/10.1038/s41598-018-32759-7

Gregory, K.E., LaPlante, R.D., Shan, G., Kumar, D.V., Gregas, M., 2015. Mode of birth influences preterm infant intestinal colonization with bacteroides over the early neonatal period. Adv Neonatal Care 15, 386-393. https://doi.org/10.1097/ANC.0000000000000237

Grenham, S., Clarke, G., Cryan, J.F., Dinan, T.G., 2011. Brain-gut-microbe communication in health and disease. Front. Physiol. 2. https://doi.org/10.3389/fphys.2011.00094

Grieneisen, L.E., Charpentier, M.J.E., Alberts, S.C., Blekhman, R., Bradburd, G., Tung, J., Archie, E.A., 2019. Genes, geology and germs: gut microbiota across a primate hybrid zone are explained by site soil properties, not host species. Proc. R. Soc. B 286, 20190431. https://doi.org/10.1098/rspb.2019.0431

Grieneisen, L.E., Livermore, J., Alberts, S., Tung, J., Archie, E.A., 2017. Group living and male dispersal predict the core gut microbiome in wild baboons. Integr Comp Biol 57, 770-785. https://doi.org/10.1093/icb/icx046

Grueter, C.C., Bissonnette, A., Isler, K., van Schaik, C.P., 2013. Grooming and group cohesion in primates: implications for the evolution of language. Evol Hum Behav 34, 61-68. https://doi.org/10.1016/j.evolhumbehav.2012.09.004

Guillou, L., Bachar, D., Audic, S., Bass, D., Berney, C., Bittner, L., Boutte, C., Burgaud, G., de Vargas, C., Decelle, J., del Campo, J., Dolan, J.R., Dunthorn, M., Edvardsen, B., Holzmann, M., Kooistra, W.H.C.F., Lara, E., Le Bescot, N., Logares, R., Mahé, F., Massana, R., Montresor, M., Morard, R., Not, F., Pawlowski, J., Probert, I., Sauvadet, A.-L., Siano, R., Stoeck, T., Vaulot, D., Zimmermann, P., Christen, R., 2013. The protist ribosomal reference database (PR2): A catalog of unicellular eukaryote small sub-unit rRNA sequences with curated taxonomy. Nucleic Acids Res 41, D597-D604. https://doi.org/10.1093/nar/gks1160

Gust, D.A., Gordon, T.P., Hambright, M.K., Wilson, M.E., 1993. Relationship between social factors and pituitary-adrenocortical activity in female rhesus monkeys (Macaca mulatta). Horm Behav 27, 318-331. https://doi.org/10.1006/hbeh.1993.1024

Gustison, M.L., MacLarnon, A., Wiper, S., Semple, S., 2012. An experimental study of behavioural coping strategies in freeranging female Barbary macaques (Macaca sylvanus). Stress 15, 608-617. https://doi.org/10.3109/10253890.2012.668589

Habig, B., Archie, E.A., 2015. Social status, immune response and parasitism in males: a meta-analysis. Proc. R. Soc. B 370, 20140109. https://doi.org/10.1098/rstb.2014.0109

Habig, B., Doellman, M.M., Woods, K., Olansen, J., Archie, E.A., 2018. Social status and parasitism in male and female vertebrates: a meta-analysis. Sci Rep 8, 3629. https://doi.org/10.1038/s41598-018-21994-7

Hadziavdic, K., Lekang, K., Lanzen, A., Jonassen, I., Thompson, E.M., Troedsson, C., 2014. Characterization of the $18 \mathrm{~S}$ rRNA gene for designing universal eukaryote specific primers. PLOS ONE 9, e87624. https://doi.org/10.1371/journal.pone.0087624

Hämäläinen, A., Heistermann, M., Kraus, C., 2015. The stress of growing old: Sex- and season-specific effects of age on allostatic load in wild grey mouse lemurs. Oecologia 178, 1063-1075. https://doi.org/10.1007/s00442-015-3297-3

Hamilton, W.D., 1971. Geometry for the selfish herd. J Theo Biol 31, 295-311. https://doi.org/10.1016/0022-5193(71)90189-5

Harris, B.N., Carr, J.A., 2016. The role of the hypothalamus-pituitary-adrenal/interrenal axis in mediating predator-avoidance trade-offs. Gen Comp Endocrinol 230-231, 110-142. https://doi.org/10.1016/j.ygcen.2016.04.006

Hart, B.L., 2011. Behavioural defences in animals against pathogens and parasites: parallels with the pillars of medicine in humans. Proc. R. Soc. B 366, 3406-3417. https://doi.org/10.1098/rstb.2011.0092

Hart, B.L., Hart, L.A., Mooring, M.S., Olubayo, R., 1992. Biological basis of grooming behaviour in antelope: the body-size, vigilance and habitat principles. Anim Behav 44, 615-631. https://doi.org/10.1016/S0003-3472(05)80290-8

Hawlena, H., Bashary, D., Abramsky, Z., Krasnov, B.R., 2007. Benefits, costs and constraints of anti-parasitic grooming in adult and juvenile rodents. Ethology 113, 394-402. https://doi.org/10.1111/j.1439-0310.2007.01332.x

Heistermann, M., Ademmer, C., Kaumanns, W., 2004. Ovarian cycle and effect of social changes on adrenal and ovarian function in Pygathrix nemaeus. Int J Primatol 25, 689-708. https://doi.org/10.1023/B:IJOP.0000023581.17889.0f 
Heistermann, M., Palme, R., Ganswindt, A., 2006. Comparison of different enzymeimmunoassays for assessment of adrenocortical activity in primates based on fecal analysis. Am J Primatol 68, 257-273. https://doi.org/10.1002/ajp.20222

Heitlinger, E., Ferreira, S.C.M., Thierer, D., Hofer, H., East, M.L., 2017. The intestinal eukaryotic and bacterial biome of Spotted hyenas: The impact of social status and age on diversity and composition. Front. Cell. Infect. Microbiol. 7. https://doi.org/10.3389/fcimb.2017.00262

Hennessy, M.B., Hornschuh, G., Kaiser, S., Sachser, N., 2006. Cortisol responses and social buffering: A study throughout the life span. Horm Behav 49, 383-390. https://doi.org/10.1016/j.yhbeh.2005.08.006

Higham, J.P., 2016. Field endocrinology of nonhuman primates: past, present, and future. Horm Behav 84, 145-155. https://doi.org/10.1016/j.yhbeh.2016.07.001

Higham, J.P., Kraus, C., Stahl-Hennig, C., Engelhardt, A., Fuchs, D., Heistermann, M., 2015. Evaluating noninvasive markers of nonhuman primate immune activation and inflammation. Am J Phys Anthropol 158, 673-684. https://doi.org/10.1002/ajpa.22821

Higham, J.P., MacLarnon, A.M., Heistermann, M., Ross, C., Semple, S., 2009. Rates of self-directed behaviour and faecal glucocorticoid levels are not correlated in female wild olive baboons (Papio hamadryas anubis). Stress 12, 526-532. https://doi.org/10.3109/10253890902756565

Hill, D.A., Okayasu, N., 1995. Absence of "Youngest Ascendancy" in the dominance relations of sisters in wild Japanese macaques (Macaca Fuscata Yakui). Behaviour 132, 367-379. https://doi.org/10.1163/156853995X00612

Holand, Ø., Gjøstein, H., Losvar, A., Kumpula, J., Smith, M.E., Røed, K.H., Nieminen, M., Weladji, R.B., 2004. Social rank in female reindeer (Rangifer tarandus): Effects of body mass, antler size and age. J Zool 263, 365-372. https://doi.org/10.1017/S0952836904005382

Holt-Lunstad, J., Smith, T.B., Layton, J.B., 2010. Social relationships and mortality risk: A meta-analytic review. PLOS Medicine 7 , e1000316. https://doi.org/10.1371/journal.pmed.1000316

Hooper, L.V., Littman, D.R., Macpherson, A.J., 2012. Interactions between the microbiota and the immune system. Science 336, 1268-1273. https://doi.org/10.1126/science.1223490

House, J.S., Landis, K.R., Umberson, D., 1988. Social relationships and health. Science 241, 540-545. https://doi.org/10.1126/science.3399889

Houser, D.S., Yeates, L.C., Crocker, D.E., 2011. Cold stress induces an adrenocortical response in bottlenose dolphins (Tursiops truncatus). J. Zoo Wildl. Med. 42, 565-571. https://doi.org/10.1638/2010-0121.1

Hrdy, S.B., 2009. The Woman That Never Evolved. Harvard University Press.

Huber, M., Knottnerus, J.A., Green, L., Horst, H. van der, Jadad, A.R., Kromhout, D., Leonard, B., Lorig, K., Loureiro, M.I., Meer, J.W.M. van der, Schnabel, P., Smith, R., Weel, C. van, Smid, H., 2011. How should we define health? BMJ 343. https://doi.org/10.1136/bmj.d4163

Huffman, M.A., 2003. Animal self-medication and ethno-medicine: exploration and exploitation of the medicinal properties of plants. Proc Nutr Soc 62, 371-381

Huffman, M.A., 1997. Current evidence for self-medication in primates: A multidisciplinary perspective. Am J Phys Anthropol 104, 171-200. https://doi.org/10.1002/(SICI)1096-8644(1997)25+<171::AID-AJPA7>3.0.CO;2-7

Huffman, M.A., Chapman, C.A., 2009. Primate parasite ecology. Cambridge University Press.

Hughes, W.O.H., Eilenberg, J., Boomsma, J.J., 2002. Trade-offs in group living: transmission and disease resistance in leafcutting ants. Proc R Soc Lond [Biol] 269, 1811-1819. https://doi.org/10.1098/rspb.2002.2113

Hunt, K.E., Rolland, R.M., Kraus, S.D., Wasser, S.K., 2006. Analysis of fecal glucocorticoids in the North Atlantic right whale (Eubalaena glacialis). Gen Comp Endocrinol 148, 260-272. https://doi.org/10.1016/j.ygcen.2006.03.012

Hutchins, M., Barash, D.P., 1976. Grooming in primates: Implications for its utilitarian function. Primates 17, 145-150. https://doi.org/10.1007/BF02382848

Irwin, M.T., Raharison, J.-L., 2009. A review of the endoparasites of the lemurs of Madagascar. Malagasy Nature 66-93.

Iwamoto, T., Dunbar, R.I.M., 1983. Thermoregulation, habitat quality and the behavioural ecology of Gelada baboons. Journal of Animal Ecology 52, 357-366. https://doi.org/10.2307/4559

Jambroes, M., Nederland, T., Kaljouw, M., van Vliet, K., Essink-Bot, M.-L., Ruwaard, D., 2016. Implications of health as 'the ability to adapt and self-manage' for public health policy: a qualitative study. Eur J Public Health 26, 412-416. https://doi.org/10.1093/eurpub/ckv206

Janson, C.H., Goldsmith, M.L., 1995. Predicting group size in primates: foraging costs and predation risks. Behavioral Ecology 6 , 326-336. https://doi.org/10.1093/beheco/6.3.326

Janson, C.H., van Schaik, C.P., 1988. Recognizing the many faces of primate food competition: Methods. Behaviour 105, 165186.

Jessop, T.S., Lane, M.L., Teasdale, L., Stuart-Fox, D., Wilson, R.S., Careau, V., Moore, I.T., 2016. Multiscale evaluation of thermal dependence in the glucocorticoid response of vertebrates. Am Nat 188, 342-356. https://doi.org/10.1086/687588

Jimeno, B., Hau, M., Verhulst, S., 2018. Glucocorticoid-temperature association is shaped by foraging costs in individual zebra finches. J Exp Biol 221, jeb187880. https://doi.org/10.1242/jeb.187880

Jimeno, B., Hau, M., Verhulst, S., 2017. Strong association between corticosterone levels and temperature-dependent metabolic rate in individual zebra finches. J Exp Biol 220, 4426-4431. https://doi.org/10.1242/jeb.166124

Jolly, A., 1984. The puzzle of female feeding priority. Female Primates : Studies by Women Primatologists.

Jolly, A., 1966. Lemur Social Behavior and Primate Intelligence. Science 153, 501-506. https://doi.org/10.1126/science.153.3735.501 
Jolly, A., Gustafson, H., Oliver, W.L.R., O'Connor, S.M., 1982. Propithecus verreauxi population and ranging at Berenty, Madagascar, 1975 and 1980. Folia Primatol. 39, 124-144. https://doi.org/10.1159/000156071

Junge, R.E., Barrett, M.A., Yoder, A.D., 2011. Effects of anthropogenic disturbance on indri (Indri indri) health in Madagascar. Am J Primatol 73, 632-642. https://doi.org/10.1002/ajp.20938

Jungwirth, A., Taborsky, M., 2015. First- and second-order sociality determine survival and reproduction in cooperative cichlids. Proc. R. Soc. B 282, 20151971. https://doi.org/10.1098/rspb.2015.1971

Kappeler, P.M., 2017. Sex roles and adult sex ratios: insights from mammalian biology and consequences for primate behaviour. Proc. R. Soc. B 372, 20160321. https://doi.org/10.1098/rstb.2016.0321

Kappeler, P.M., 1998. To whom it may concern: The transmission and function of chemical signals in Lemur catta. Behav Ecol Sociobiol 42, 411-421. https://doi.org/10.1007/s002650050455

Kappeler, P.M., 1997. Determinants of primate social organization: Comparative evidence and new insights from Malagasy lemurs. Biol Rev Camb Philos Soc 72, 111-151.

Kappeler, P.M., 1993. Sexual selection and lemur social systems, in: Kappeler, P.M., Ganzhorn, J.U. (Eds.), Lemur Social Systems and Their Ecological Basis. Springer US, Boston, MA, pp. 223-240. https://doi.org/10.1007/978-1-4899-2412-4_16

Kappeler, P.M., Cremer, S., Nunn, C.L., 2015. Sociality and health: Impacts of sociality on disease susceptibility and transmission in animal and human societies. Phil. Trans. R. Soc. B 370, 20140116. https://doi.org/10.1098/rstb.2014.0116

Kappeler, P.M., Fichtel, C., 2015. Eco-evo-devo of the lemur syndrome: Did adaptive behavioral plasticity get canalized in a large primate radiation? Front Zool12, 1-16. https://doi.org/10.1186/1742-9994-12-S1-S15

Kappeler, P.M., Fichtel, C., 2012. A 15-year perspective on the social organization and life history of sifaka in Kirindy forest, in: Kappeler, P.M., Watts, D.P. (Eds.), Long-Term Field Studies of Primates. Springer Berlin, pp. 101-121. https://doi.org/10.1007/978-3-642-22514-7_5

Kappeler, P.M., Heymann, E.W., 1996. Nonconvergence in the evolution of primate life history and socio-ecology. Biol J Linn Soc 59, 297-326. https://doi.org/10.1111/j.1095-8312.1996.tb01468.x

Kappeler, P.M., Mass, V., Port, M., 2009a. Even adult sex ratios in lemurs: Potential costs and benefits of subordinate males in Verreaux's sifaka (Propithecus verreauxi) in the Kirindy Forest CFPF, Madagascar. Am J Phys Anthropol 140, 487-497. https://doi.org/10.1002/ajpa.21091

Kappeler, P.M., Mass, V., Port, M., 2009b. Even adult sex ratios in lemurs: Potential costs and benefits of subordinate males in Verreaux's Sifaka (Propithecus verreauxi) in the Kirindy Forest CFPF, Madagascar. Am J Phys Anthropol 140, 487-497.

Kappeler, P.M., Nunn, C.L., Vining, A.Q., Goodman, S.M., 2019. Evolutionary dynamics of sexual size dimorphism in non-volant mammals following their independent colonization of Madagascar. Sci Rep 9, 1-14. https://doi.org/10.1038/s41598018-36246-x

Kappeler, P.M., Schäffler, L., 2007. The lemur syndrome unresolved: Extreme male reproductive skew in sifakas (Propithecus verreauxi), a sexually monomorphic primate with female dominance. Behav Ecol Sociobiol 62, 1007-1015. https://doi.org/10.1007/s00265-007-0528-6

Kappeler, P.M., van Schaik, C.P., 2002. Evolution of primate social systems. Int J Primatol 23, 707-740. https://doi.org/10.1023/A:1015520830318

Kavana, T.S., Erinjery, J.J., Singh, M., 2015. Folivory as a constraint on social behaviour of langurs in South India. FPR 86, 420431. https://doi.org/10.1159/000438990

Keiser, C.N., Pinter-Wollman, N., Ziemba, M.J., Kothamasu, K.S., Pruitt, J.N., 2018. The primary case is not enough: Variation among individuals, groups and social networks modify bacterial transmission dynamics. J Anim Ecol 87, 369-378. https://doi.org/10.1111/1365-2656.12729

Kinross, J.M., Darzi, A.W., Nicholson, J.K., 2011. Gut microbiome-host interactions in health and disease. Genome Medicine 3 , 14. https://doi.org/10.1186/gm228

Klindworth, A., Pruesse, E., Schweer, T., Peplies, J., Quast, C., Horn, M., Glöckner, F.O., 2013. Evaluation of general 16S ribosomal RNA gene PCR primers for classical and next-generation sequencing-based diversity studies. Nucleic Acids Res 41, e1-e1. https://doi.org/10.1093/nar/gks808

Knights, D., Lassen, K.G., Xavier, R.J., 2013. Advances in inflammatory bowel disease pathogenesis: linking host genetics and the microbiome. Gut 62, 1505-1510. https://doi.org/10.1136/gutjnl-2012-303954

Koch, F., Ganzhorn, J.U., Rothman, J.M., Chapman, C.A., Fichtel, C., 2017. Sex and seasonal differences in diet and nutrient intake in Verreaux's sifakas (Propithecus verreauxi). Am J Primatol 79, e22595. https://doi.org/10.1002/ajp.22595

Koch, F., Signer, J., Kappeler, P.M., Fichtel, C., 2016a. The role of the residence-effect on the outcome of intergroup encounters in Verreaux's sifakas. Sci Rep 6, 28457. https://doi.org/10.1038/srep28457

Koch, F., Signer, J., Kappeler, P.M., Fichtel, C., 2016b. Intergroup encounters in Verreaux's sifakas (Propithecus verreauxi): Who fights and why? Behav Ecol Sociobiol 70, 797-808. https://doi.org/10.1007/s00265-016-2105-3

Koch, H., Schmid-Hempel, P., 2011. Socially transmitted gut microbiota protect bumble bees against an intestinal parasite. PNAS 108, 19288-19292. https://doi.org/10.1073/pnas.1110474108

Koenig, A., 2002. Competition for resources and its behavioral consequences among female primates. Int J Primatol 23, 759783. https://doi.org/10.1023/A:1015524931226

Koenig, A., Scarry, C.J., Wheeler, B.C., Borries, C., 2013. Variation in grouping patterns, mating systems and social structure: What socio-ecological models attempt to explain. Phil. Trans. R. Soc. B 368, 20120348. https://doi.org/10.1098/rstb.2012.0348

Kohl, K.D., Brun, A., Magallanes, M., Brinkerhoff, J., Laspiur, A., Acosta, J.C., Caviedes-Vidal, E., Bordenstein, S.R., 2017. Gut microbial ecology of lizards: Insights into diversity in the wild, effects of captivity, variation across gut regions and transmission. Mol Ecol 26, 1175-1189. https://doi.org/10.1111/mec.13921 
Kolodny, O., Weinberg, M., Reshef, L., Harten, L., Hefetz, A., Gophna, U., Feldman, M.W., Yovel, Y., 2019. Coordinated change at the colony level in fruit bat fur microbiomes through time. Nat Ecol Evol 3, 116-124. https://doi.org/10.1038/s41559-018-0731-z

Konrad, M., Vyleta, M.L., Theis, F.J., Stock, M., Tragust, S., Klatt, M., Drescher, V., Marr, C., Ugelvig, L.V., Cremer, S., 2012. Social transfer of pathogenic fungus promotes active immunisation in ant colonies. PLOS Biol 10, e1001300. https://doi.org/10.1371/journal.pbio.1001300

Korstjens, A.H., Lehmann, J., Dunbar, R.I.M., 2010. Resting time as an ecological constraint on primate biogeography. Anim Behav 79, 361-374. https://doi.org/10.1016/j.anbehav.2009.11.012

Korstjens, A.H., Verhoeckx, I.L., Dunbar, R.I.M., 2006. Time as a constraint on group size in spider monkeys. Behav Ecol Sociobiol 60, 683. https://doi.org/10.1007/s00265-006-0212-2

Kotrschal, K., Hirschenhauser, K., Möstl, E., 1998. The relationship between social stress and dominance is seasonal in greylag geese. Animal Behaviour 55, 171-176. https://doi.org/10.1006/anbe.1997.0597

Kovacs, A., Ben-Jacob, N., Tayem, H., Halperin, E., Iraqi, F.A., Gophna, U., 2011. Genotype is a stronger determinant than sex of the mouse gut microbiota. Microb Ecol 61, 423-428. https://doi.org/10.1007/s00248-010-9787-2

Kraus, C., Heistermann, M., Kappeler, P.M., 1999. Physiological suppression of sexual function of subordinate males: A subtle form of intrasexual competition among male sifakas (Propithecus verreauxi)? Phys Behav 66, 855-861. https://doi.org/10.1016/S0031-9384(99)00024-4

Krause, J., Ruxton, G.D., 2002. Living in Groups. Oxford University Press.

Kulahci, I.G., Rubenstein, D.I., Ghazanfar, A.A., 2015. Lemurs groom-at-a-distance through vocal networks. Anim Behav 110, 179-186. https://doi.org/10.1016/j.anbehav.2015.09.016

Kulkarni, S., Heeb, P., 2007. Social and sexual behaviours aid transmission of bacteria in birds. Behav Proc74, 88-92. https://doi.org/10.1016/j.beproc.2006.10.005

Kuthyar, S., Manus, M.B., Amato, K.R., 2019. Leveraging non-human primates for exploring the social transmission of microbes. Curr Opin Microbiol 50, 8-14. https://doi.org/10.1016/j.mib.2019.09.001

Kutsukake, N., Clutton-Brock, T.H., 2006. Social functions of allogrooming in cooperatively breeding meerkats. Anim Behav 72, 1059-1068. https://doi.org/10.1016/j.anbehav.2006.02.016

Landys, M.M., Ramenofsky, M., Wingfield, J.C., 2006. Actions of glucocorticoids at a seasonal baseline as compared to stressrelated levels in the regulation of periodic life processes. Gen Comp Endocrinol 148, 132-149. https://doi.org/10.1016/j.ygcen.2006.02.013

Lane, J.E., Boutin, S., Speakman, J.R., Humphries, M.M., 2010. Energetic costs of male reproduction in a scramble competition mating system. J Anim Ecol 79, 27-34. https://doi.org/10.1111/j.1365-2656.2009.01592.x

Lawler, R.R., Richard, A.F., Riley, M.A., 2005. Intrasexual selection in Verreaux's sifaka (Propithecus verreauxi verreauxi). J Hum Evol 48, 259-277. https://doi.org/10.1016/j.jhevol.2004.11.005

Lax, S., Smith, D.P., Hampton-Marcell, J., Owens, S.M., Handley, K.M., Scott, N.M., Gibbons, S.M., Larsen, P., Shogan, B.D., Weiss, S., Metcalf, J.L., Ursell, L.K., Vázquez-Baeza, Y., Treuren, W.V., Hasan, N.A., Gibson, M.K., Colwell, R., Dantas, G., Knight, R., Gilbert, J.A., 2014. Longitudinal analysis of microbial interaction between humans and the indoor environment. Science 345, 1048-1052. https://doi.org/10.1126/science.1254529

Lea, J.M.D., Walker, S.L., Kerley, G.I.H., Jackson, J., Matevich, S.C., Shultz, S., 2018. Non-invasive physiological markers demonstrate link between habitat quality, adult sex ratio and poor population growth rate in a vulnerable species, the Cape mountain zebra. Funct Ecol 32, 300-312. https://doi.org/10.1111/1365-2435.13000

Lehmann, J., Korstjens, A.H., Dunbar, R.I.M., 2007. Group size, grooming and social cohesion in primates. Anim Behav 74, 1617-1629. https://doi.org/10.1016/j.anbehav.2006.10.025

Leimberger, K.G., Lewis, R.J., 2015. Patterns of male dispersal in Verreaux's sifaka (Propithecus verreauxi) at Kirindy Mitea National Park. Am J Primatol 79, e22455. https://doi.org/10.1002/ajp.22455

Levin, I., Zonana, D.M., Fosdick, B.K., Song, S.J., Knight, R., Safran, R.J., 2016. Stress response, gut microbial diversity and sexual signals correlate with social interactions. Biol Lett 12, 20160352. https://doi.org/10.1098/rsbl.2016.0352

Lewis, R. j., Kappeler, P. m., 2005. Seasonality, body condition, and timing of reproduction in Propithecus verreauxi verreauxi in the Kirindy Forest. Am. J. Primatol. 67, 347-364. https://doi.org/10.1002/ajp.20187

Lewis, R.J., 2019. Subordination signals improve the quality of social relationships in Verreaux's Sifaka: Implications for the evolution of power structures and social complexity. Am J Phys Anthropol 169, 599-607. https://doi.org/10.1002/ajpa.23876

Lewis, R.J., 2010. Grooming patterns in Verreaux's sifaka. Am. J. Primatol. 72, 254-261. https://doi.org/10.1002/ajp.20776

Lewis, R.J., 2008. Social influences on group membership in Propithecus verreauxi verreauxi. Int J Primatol 29, 1249-1270. https://doi.org/10.1007/s10764-008-9304-3

Lewis, R.J., 2005. Sex differences in scent-marking in sifaka: Mating conflict or male services? Am. J. Phys. Anthropol. 128, 389398. https://doi.org/10.1002/ajpa.20206

Lewis, R.J., Razafindrasamba, S.M., Tolojanahary, J.P., 2003a. Observed infanticide in a seasonal breeding prosimian (Propithecus verreauxi verreauxi) in Kirindy Forest, Madagascar. FPR 74, 101-103. https://doi.org/10.1159/000070006

Lewis, R.J., Razafindrasamba, S.M., Tolojanahary, J.P., 2003b. Observed infanticide in a seasonal breeding prosimian (Propithecus verreauxi verreauxi) in Kirindy Forest, Madagascar. Folia Primatologica 74.

Ley, R.E., Bäckhed, F., Turnbaugh, P., Lozupone, C.A., Knight, R.D., Gordon, J.I., 2005. Obesity alters gut microbial ecology. PNAS 102, 11070-11075. https://doi.org/10.1073/pnas.0504978102 
Li, H., Qu, J., Li, T., Li, J., Lin, Q., Li, X., 2016. Pika population density is associated with the composition and diversity of gut microbiota. Front. Microbiol. 7. https://doi.org/10.3389/fmicb.2016.00758

Li, K., Bihan, M., Methé, B.A., 2013. Analyses of the stability and core taxonomic memberships of the human microbiome. PLOS ONE 8, e63139. https://doi.org/10.1371/journal.pone.0063139

Lima, S.L., 1996. The influence of models in the interpretation of vigilance, in: Allen, C., Jamison, D. (Eds.), Readings in Animal Cognition. MIT Press, pp. 201-216.

Lombardo, M.P., 2008. Access to mutualistic endosymbiotic microbes: an underappreciated benefit of group living. Behav Ecol Sociobiol 62, 479-497. https://doi.org/10.1007/s00265-007-0428-9

Loudon, J.E., Sauther, M.L., 2013. Verreaux's sifaka (Propithecus verreauxi) and ring-tailed lemur (Lemur catta) endoparasitism at the Bezà Mahafaly Special Reserve. MCD 8, 21-28. https://doi.org/10.4314/mcd.v8i1.4

Lozupone, C.A., Stombaugh, J.I., Gordon, J.I., Jansson, J.K., Knight, R., 2012. Diversity, stability and resilience of the human gut microbiota. Nature 489, 220-230. https://doi.org/10.1038/nature11550

Lukas, D., Clutton-Brock, T.H., 2013. The evolution of social monogamy in mammals. Science 341, 526-530. https://doi.org/10.1126/science.1238677

Lynch, J.W., Ziegler, T.E., Strier, K.B., 2002. Individual and seasonal variation in fecal testosterone and cortisol levels of wild male Tufted capuchin monkeys, Cebus apella nigritus. Horm Behav 41, 275-287. https://doi.org/10.1006/hbeh.2002.1772

Lynn, S.E., Stamplis, T.B., Barrington, W.T., Weida, N., Hudak, C.A., 2010. Food, stress, and reproduction: Short-term fasting alters endocrine physiology and reproductive behavior in the zebra finch. Horm Behav 58, 214-222. https://doi.org/10.1016/j.yhbeh.2010.03.015

MacDougall-Shackleton, S.A., Bonier, F., Romero, L.M., Moore, I.T., 2019. Glucocorticoids and "stress" are not synonymous. Integr Org Biol 1. https://doi.org/10.1093/iob/obz017

MacIntosh, A.J.J., Jacobs, A., Garcia, C., Shimizu, K., Mouri, K., Huffman, M.A., Hernandez, A.D., 2012. Monkeys in the middle: Parasite transmission through the social network of a wild primate. PLOS ONE 7, e51144. https://doi.org/10.1371/journal.pone.0051144

Madden, J.R., Clutton-Brock, T.H., 2009. Manipulating grooming by decreasing ectoparasite load causes unpredicted changes in antagonism. Proc. R. Soc. B 276, 1263-1268. https://doi.org/10.1098/rspb.2008.1661

Maestripieri, D., 1993. Vigilance costs of allogrooming in macaquemothers. Am Nat 141, 744-753. https://doi.org/10.1086/285503

Majolo, B., Huang, P., 2018. Group Living, in: Vonk, J., Shackelford, T. (Eds.), Encyclopedia of Animal Cognition and Behavior. Springer International Publishing, Cham, pp. 1-12. https://doi.org/10.1007/978-3-319-47829-6_1865-1

Maréchal, L., Semple, S., Majolo, B., Qarro, M., Heistermann, M., MacLarnon, A., 2011. Impacts of tourism on anxiety and physiological stress levels in wild male Barbary macaques. Biol Conserv 144, 2188-2193. https://doi.org/10.1016/j.biocon.2011.05.010

Markham, A.C., Gesquiere, L.R., Alberts, S.C., Altmann, J., 2015. Optimal group size in a highly social mammal. P Natl Acad Sci USA 112, 14882-14887. https://doi.org/10.1073/pnas.1517794112

Markham, A.C., Gould, L., 2018. Diet and behaviour of adult Propithecus verreauxi (Verreaux's sifaka) in Southern Madagascar during the birth season. Lemur News 21.

Markham, A.C., Santymire, R.M., Lonsdorf, E.V., Heintz, M.R., Lipende, I., Murray, C.M., 2014. Rank effects on social stress in lactating chimpanzees. Anim Behav 87, 195-202. https://doi.org/10.1016/j.anbehav.2013.10.031

Martens, E.C., Kelly, A.G., Tauzin, A.S., Brumer, H., 2014. The devil lies in the details: How variations in polysaccharide finestructure impact the physiology and evolution of gut microbes. J Mol Biol, 426, 3851-3865. https://doi.org/10.1016/j.jmb.2014.06.022

Martin, M., 2011. Cutadapt removes adapter sequences from high-throughput sequencing reads. EMBnet.journal 17, 10-12. https://doi.org/10.14806/ej.17.1.200

Martinson, V.G., Danforth, B.N., Minckley, R.L., Rueppell, O., Tingek, S., Moran, N.A., 2011. A simple and distinctive microbiota associated with honey bees and bumble bees. Mol Ecol 20, 619-628. https://doi.org/10.1111/j.1365294X.2010.04959.x

Marty, P.R., Beisner, B., Kaburu, S.S.K., Balasubramaniam, K., Bliss-Moreau, E., Ruppert, N., Mohd Sah, S.A., Ismail, A., Arlet, M.E., Atwill, E.R., McCowan, B., 2019. Time constraints imposed by anthropogenic environments alter social behaviour in longtailed macaques. Anim Behav 150, 157-165. https://doi.org/10.1016/j.anbehav.2019.02.010

Mass, V., Heistermann, M., Kappeler, P.M., 2009. Mate-guarding as a male reproductive tactic in Propithecus verreauxi. Int J Primatol 30, 389-409. https://doi.org/10.1007/s10764-009-9345-2

Mateo, J.M., 2007. Ecological and hormonal correlates of antipredator behavior in adult Belding's ground squirrels (Spermophilus beldingi). Behav Ecol Sociobiol 62, 37-49. https://doi.org/10.1007/s00265-007-0436-9

McCormick, S.D., Romero, L.M., 2017. Conservation endocrinology. BioScience 67, 429-442. https://doi.org/10.1093/biosci/bix026

McEwen, B.S., 2012. Brain on stress: How the social environment gets under the skin. PNAS 109, 17180-17185. https://doi.org/10.1073/pnas.1121254109

McEwen, B.S., Biron, C.A., Brunson, K.W., Bulloch, K., Chambers, W.H., Dhabhar, F.S., Goldfarb, R.H., Kitson, R.P., Miller, A.H., Spencer, R.L., Weiss, J.M., 1997. The role of adrenocorticoids as modulators of immune function in health and disease: neural, endocrine and immune interactions. Brain Res Brain Res Rev 23, 79-133. https://doi.org/10.1016/S0165-0173(96)00012-4 
McEwen, B.S., Wingfield, J.C., 2003. The concept of allostasis in biology and biomedicine. Horm Behav 43, 2-15. https://doi.org/10.1016/S0018-506X(02)00024-7

McFarland, R., Henzi, S.P., Barrett, L., Wanigaratne, A., Coetzee, E., Fuller, A., Hetem, R.S., Mitchell, D., Maloney, S.K., 2016. Thermal consequences of increased pelt loft infer an additional utilitarian function for grooming. Am J Primatol 78, 456-461. https://doi.org/10.1002/ajp.22519

McFarland, R., Murphy, D., Lusseau, D., Henzi, S.P., Parker, J.L., Pollet, T.V., Barrett, L., 2017. The 'strength of weak ties' among female baboons: Fitness-related benefits of social bonds. Anim Behavr 126, 101-106. https://doi.org/10.1016/j.anbehav.2017.02.002

Meadow, J.F., Altrichter, A.E., Bateman, A.C., Stenson, J., Brown, G.Z., Green, J.L., Bohannan, B.J.M., 2015. Humans differ in their personal microbial cloud. PeerJ 3, e1258. https://doi.org/10.7717/peerj.1258

Metcalfe, N.B., 1986. Intraspecific variation in competitive ability and food intake in salmonids: consequences for energy budgets and growth rates. J Fish Biol 28, 525-531. https://doi.org/10.1111/j.1095-8649.1986.tb05190.x

Meunier, J., 2015. Social immunity and the evolution of group living in insects. Proc. R. Soc. B 370, 20140102. https://doi.org/10.1098/rstb.2014.0102

Mitani, J.C., Call, J., Kappeler, P.M., Palombit, R.A., Silk, J.B., 2012. The Evolution of Primate Societies. University of Chicago Press.

Mittermeier, R.A., Ganzhorn, J.U., Konstant, W.R., Glander, K., Tattersall, I., Groves, C.P., Rylands, A.B., Hapke, A., Ratsimbazafy, J., Mayor, M.I., Louis, E.E., Rumpler, Y., Schwitzer, C., Rasoloarison, R.M., 2008. Lemur Diversity in Madagascar. Int J Primatol 29, 1607-1656. https://doi.org/10.1007/s10764-008-9317-y

Moeller, A.H., Foerster, S., Wilson, M.L., Pusey, A.E., Hahn, B.H., Ochman, H., 2016. Social behavior shapes the chimpanzee pan-microbiome. Science Advances 2, e1500997. https://doi.org/10.1126/sciadv.1500997

Moeller, A.H., Suzuki, T.A., Phifer-Rixey, M., Nachman, M.W., 2018. Transmission modes of the mammalian gut microbiota. Science 362, 453-457. https://doi.org/10.1126/science.aat7164

Montiel-Castro, A.J., González-Cervantes, R.M., Bravo-Ruiseco, G., Pacheco-López, G., 2013. The microbiota-gut-brain axis: neurobehavioral correlates, health and sociality. Front Integr Neurosci 7. https://doi.org/10.3389/fnint.2013.00070

Mooring, M.S., Hart, B.L., 1995. Costs of allogrooming in impala: Distraction from vigilance. Anim Behav 49, 1414-1416. https://doi.org/10.1006/anbe.1995.0175

Morland, H.S., 1993. Seasonal Behavioral variation and its relationship to thermoregulation in Ruffed lemurs (Varecia Variegata Variegata), in: Kappeler, P.M., Ganzhorn, J.U. (Eds.), Lemur Social Systems and Their Ecological Basis. Springer US, Boston, MA, pp. 193-203. https://doi.org/10.1007/978-1-4899-2412-4_14

Morton, E.R., Lynch, J., Froment, A., Lafosse, S., Heyer, E., Przeworski, M., Blekhman, R., Ségurel, L., 2015. Variation in rural African gut microbiota Is strongly correlated with colonization by entamoeba and aubsistence. PLOS Genetics 11 , e1005658. https://doi.org/10.1371/journal.pgen.1005658

Muehlenbein, M.P., Schwartz, M., Richard, A., 2003. Parasitologic analyses of the sifaka (Propithecus verreauxi verreauxi) at Beza Mahafaly, Madagascar. J Zoo Wildlife Med 34, 274-277. https://doi.org/10.1638/10427260(2003)034[0274:PAOTSP]2.0.CO;2

Muller, M.N., Wrangham, R.W., 2004. Dominance, cortisol and stress in wild chimpanzees (Pan troglodytes schweinfurthii). Behav Ecol Sociobiol 55, 332-340. https://doi.org/10.1007/s00265-003-0713-1

Nelson, T.M., Rogers, T.L., Carlini, A.R., Brown, M.V., 2013. Diet and phylogeny shape the gut microbiota of Antarctic seals: a comparison of wild and captive animals. Env Microbiol 15, 1132-1145. https://doi.org/10.1111/1462-2920.12022

Neufeld, K.M., Kang, N., Bienenstock, J., Foster, J.A., 2011. Reduced anxiety-like behavior and central neurochemical change in germ-free mice. Neurogastroenterol Motil 23, 255-e119. https://doi.org/10.1111/j.1365-2982.2010.01620.x

Noguera, J.C., Aira, M., Pérez-Losada, M., Domínguez, J., Velando, A., 2018. Glucocorticoids modulate gastrointestinal microbiome in a wild bird. R. Soc. Open Sci. 5, 171743. https://doi.org/10.1098/rsos.171743

Norscia, I., Antonacci, D., Palagi, E., 2009. Mating first, mating more: Biological market fluctuation in a wild prosimian. PLOS ONE 4, e4679. https://doi.org/10.1371/journal.pone.0004679

Norscia, I., Carrai, V., Borgognini-Tarli, S.M., 2006. Influence of dry season and food quality and quantity on behavior and feeding strategy of Propithecus verreauxi in Kirindy, Madagascar. Int J Primatol 27, 1001-1022. https://doi.org/10.1007/s10764-006-9056-x

Norscia, I., Palagi, E., 2015. The socio-matrix reloaded: From hierarchy to dominance profile in wild lemurs. PeerJ 3, e729. https://doi.org/10.7717/peerj.729

Nunn, C., Altizer, S.M., 2006. Infectious Diseases in Primates: Behavior, Ecology and Evolution. OUP Oxford

Nunn, C.L., Altizer, S., Jones, K.E., Sechrest, W., Lively, A.E.C., 2003. Comparative tests of parasite species richness in primates. Am Nat 162, 597-614. https://doi.org/10.1086/378721

Nunn, C.L., Jordán, F., McCabe, C.M., Verdolin, J.L., Fewell, J.H., 2015. Infectious disease and group size: more than just a numbers game. Phil. Trans. R. Soc. B 370, 20140111. https://doi.org/10.1098/rstb.2014.0111

Nunn, C.L., Thrall, P.H., Kappeler, P.M., 2014. Shared resources and disease dynamics in spatially structured populations. Ecol Modell 272, 198-207. https://doi.org/10.1016/j.ecolmodel.2013.10.004

Oksanen, J., Blanchet, F., Friendly, M., Kindt, R., Legendre, P., McGlinn, D., Minchin, P.R., O’hara, R.B., Simpson, G.L., Solymos, P., Stevens, M.H.M., Szoecs, E., Wagner, H., 2016. Vegan: community ecology package. R package version 2.0-7.

Oli, M.K., Armitage, K.B., 2003. Sociality and individual fitness in yellow-bellied marmots: insights from a long-term study (1962-2001). Oecologia 136, 543-550. https://doi.org/10.1007/s00442-003-1291-7

Opstal, E.J. van, Bordenstein, S.R., 2015. Rethinking heritability of the microbiome. Science 349, 1172-1173. https://doi.org/10.1126/science.aab3958 
Ostner, J., 2002. Social thermoregulation in Redfronted lemurs (Eulemur fulvus rufus). FPR 73, 175-180. https://doi.org/10.1159/000065425

Ostner, J., Kappeler, P., Heistermann, M., 2008. Androgen and glucocorticoid levels reflect seasonally occurring social challenges in male redfronted lemurs (Eulemur fulvus rufus). Behav Ecol Sociobiol 62, 627-638. https://doi.org/10.1007/s00265-007-0487-y

Ostner, J., Schülke, O., 2018. Linking sociality to fitness in primates: A call for mechanisms, in: Naguib, M., Barrett, L., Healy, S.D., Podos, J., Simmons, L.W., Zuk, M. (Eds.), Advances in the Study of Behavior. Academic Press, pp. 127-175. https://doi.org/10.1016/bs.asb.2017.12.001

Pafčo, B., Sharma, A.K., Petrželková, K.J., Vlčková, K., Todd, A., Yeoman, C.J., Wilson, B.A., Stumpf, R., White, B.A., Nelson, K.E., Leigh, S., Gomez, A., 2019. Gut microbiome composition of wild western lowland gorillas is associated with individual age and sex factors. Am J Phys Anthropol 169, 575-585. https://doi.org/10.1002/ajpa.23842

Palagi, E., Norscia, I., 2011. Scratching around stress: Hierarchy and reconciliation make the difference in wild brown lemurs (Eulemur fulvus). Stress 14, 93-97. https://doi.org/10.3109/10253890.2010.505272

Palme, R., 2019. Non-invasive measurement of glucocorticoids: Advances and problems. Physiol Behav 199, $229-243$. https://doi.org/10.1016/j.physbeh.2018.11.021

Palmer, C., Bik, E.M., DiGiulio, D.B., Relman, D.A., Brown, P.O., 2007. Development of the human infant intestinal microbiota. PLOS Biology 5, e177. https://doi.org/10.1371/journal.pbio.0050177

Paschek, N., Müller, N., Heistermann, M., Ostner, J., Schülke, O., 2019. Subtypes of aggression and their relation to anxiety in Barbary macaques. Aggress Behav 45, 120-128. https://doi.org/10.1002/ab.21801

Pearson, B.L., Reeder, D.M., Judge, P.G., 2015. Crowding increases salivary cortisol but not self-directed behavior in captive baboons. Am J Primatol 77, 462-467. https://doi.org/10.1002/ajp.22363

Peckre, L.R., Defolie, C., Kappeler, P.M., Fichtel, C., 2018. Potential self-medication using millipede secretions in red-fronted lemurs: combining anointment and ingestion for a joint action against gastrointestinal parasites? Primates 59, 483494. https://doi.org/10.1007/s10329-018-0674-7

Pereira, M.E., Strohecker, R.A., Cavigelli, S.A., Hughes, C.L., Pearson, D.D., 1999. Metabolic strategy and social behavior in Lemuridae, in: Rakotosamimanana, B., Rasamimanana, H., Ganzhorn, J.U., Goodman, S.M. (Eds.), New Directions in Lemur Studies. Springer US, Boston, MA, pp. 93-118. https://doi.org/10.1007/978-1-4615-4705-1_6

Perez-Muñoz, M.E., Arrieta, M.-C., Ramer-Tait, A.E., Walter, J., 2017. A critical assessment of the "sterile womb" and "in utero colonization" hypotheses: Implications for research on the pioneer infant microbiome. Microbiome 5, 48. https://doi.org/10.1186/s40168-017-0268-4

Perofsky, A.C., Lewis, R.J., Abondano, L.A., Fiore, A.D., Meyers, L.A., 2017. Hierarchical social networks shape gut microbial composition in wild Verreaux's sifaka. Proc. R. Soc. B 284, 20172274. https://doi.org/10.1098/rspb.2017.2274

Perofsky, A.C., Lewis, R.J., Meyers, L.A., 2019. Terrestriality and bacterial transfer: a comparative study of gut microbiomes in sympatric Malagasy mammals. ISME J 13, 50-63. https://doi.org/10.1038/s41396-018-0251-5

Pitcher, T.J., 1983. Heuristic definitions of fish shoaling behaviour. Anim Behav 31, 611-613. https://doi.org/10.1016/S00033472(83)80087-6

Pochron, S.T., Tucker, W.T., Wright, P.C., 2004. Demography, life history, and social structure in Propithecus diadema edwardsi from 1986-2000 in Ranomafana National Park, Madagascar. Am J Phys Anthropol 125, 61-72. https://doi.org/10.1002/ajpa.10266

Pochron, S.T., Wright, P.C., 2003. Variability in adult group compositions of a prosimian primate. Behav Ecol Sociobiol 54, 285293. https://doi.org/10.1007/s00265-003-0634-z

Poirotte, C., Basset, D., Willaume, E., Makaba, F., Kappeler, P.M., Charpentier, M.J.E., 2016. Environmental and individual determinants of parasite richness across seasons in a free-ranging population of Mandrills (Mandrillus sphinx). Am. J. Phys. Anthropol. 159, 442-456. https://doi.org/10.1002/ajpa.22888

Polderman, A.M., Blotkamp, J., 1995. Oesophagostomum infections in humans. Parasitol Today 11, 451-456. https://doi.org/10.1016/0169-4758(95)80058-1

Pollard, K.A., Blumstein, D.T., 2008. Time allocation and the evolution of group size. Anim Behav 76, 1683-1699. https://doi.org/10.1016/j.anbehav.2008.08.006

Poole, J.H., 1989. Announcing intent: The aggressive state of musth in African elephants. Anim Behav 37, 140-152. https://doi.org/10.1016/0003-3472(89)90014-6

Poulin, R., 2018. Modification of host social networks by manipulative parasites. Behaviour 155, 671-688. https://doi.org/10.1163/1568539X-00003456

Pribbenow, S., Shrivastav, T.G., Dehnhard, M., 2017. Measuring fecal testosterone metabolites in spotted hyenas: Choosing the wrong assay may lead to erroneous results. J Immunoassay Immunochem 38, 308-321. https://doi.org/10.1080/15321819.2016.1260584

Pride, R.E., 2005. High faecal glucocorticoid levels predict mortality in ring-tailed lemurs (Lemur catta). Biology Letters 1, 60-63. https://doi.org/10.1098/rsbl.2004.0245

Quast, C., Pruesse, E., Yilmaz, P., Gerken, J., Schweer, T., Yarza, P., Peplies, J., Glöckner, F.O., 2013. The SILVA ribosomal RNA gene database project: Improved data processing and web-based tools. Nucleic Acids Res 41, D590-D596. https://doi.org/10.1093/nar/gks1219

Quinn, G.P., Keough, M.J., 2002. Experimental Design and Data Analysis for Biologists. Cambridge University Press.

R Core Team, 2018. R: A language and environment for statistical computing. R Foundation for Statistical Computing, Vienna, Austria. 
Radford, A.N., Du Plessis, M.A., 2006. Dual function of allopreening in the cooperatively breeding green woodhoopoe, Phoeniculus purpureus. Behav Ecol Sociobiol 61, 221-230. https://doi.org/10.1007/s00265-006-0253-6

Raouf, S.A., Smith, L.C., Brown, M.B., Wingfield, J.C., Brown, C.R., 2006. Glucocorticoid hormone levels increase with group size and parasite load in cliff swallows. Anim Behav 71, 39-48. https://doi.org/10.1016/j.anbehav.2005.03.027

Rasambainarivo, F.T., Junge, R.E., Lewis, R.J., 2014. Biomedical evaluation of Verreaux's Sifaka (Propithecus verreauxi) from Kirindy Mitea National Park in Madagascar. J Zoo Wildlife Med 45, 247-255. https://doi.org/10.1638/2013-0038R1.1

Rasolonjatovo, S.M., Irwin, M.T., 2019. Exploring social dominance in wild Diademed sifakas (Propithecus diadema): Females are dominant, but It Is subtle and the benefits are not clear. FPR 1-14. https://doi.org/10.1159/000503345

Raulo, A., Dantzer, B., 2018. Associations between glucocorticoids and sociality across a continuum of vertebrate social behavior. Ecol Evol 8, 7697-7716. https://doi.org/10.1002/ece3.4059

Raulo, A., Ruokolainen, L., Lane, A., Amato, K., Knight, R., Leigh, S., Stumpf, R., White, B., Nelson, K.E., Baden, A.L., Tecot, S.R., 2017. Social behaviour and gut microbiota in red-bellied lemurs (Eulemur rubriventer): In search of the role of immunity in the evolution of sociality. J Anim Ecol 87, 388-399. https://doi.org/10.1111/1365-2656.12781

Reese, A.T., Dunn, R.R., 2018. Drivers of microbiome biodiversity: A review of general rules, feces, and ignorance. mBio 9. https://doi.org/10.1128/mBio.01294-18

Ren, T., Boutin, S., Humphries, M.M., Dantzer, B., Gorrell, J.C., Coltman, D.W., McAdam, A.G., Wu, M., 2017. Seasonal, spatial, and maternal effects on gut microbiome in wild red squirrels. Microbiome 5, 163. https://doi.org/10.1186/s40168017-0382-3

Ren, T., Grieneisen, L.E., Alberts, S.C., Archie, E.A., Wu, M., 2016. Development, diet and dynamism: longitudinal and crosssectional predictors of gut microbial communities in wild baboons. EnvironmMicrobiol18, 1312-1325. https://doi.org/10.1111/1462-2920.12852

Richard, A.F., 1992. Aggressive competition between males, female-controlled polygyny and sexual monomorphism in a Malagasy primate, Propithecus verreauxi. J Human Evol 22, 395-406. https://doi.org/10.1016/0047-2484(92)90067-J

Richard, A.F., 1985. Social boundaries in a Malagasy prosimian, the sifaka (Propithecus verreauxi). Int J Primatol 6, 553-568. https://doi.org/10.1007/BF02692288

Richard, A.F., Dewar, R.E., Schwartz, M., Ratsirarson, J., 2002. Life in the slow lane? Demography and life histories of male and female sifaka (Propithecus verreauxi verreauxi). J Zool 256, 421-436. https://doi.org/10.1017/S0952836902000468

Rifkin, J.L., Nunn, C.L., Garamszegi, L.Z., 2012. Do animals living in larger groups experience greater parasitism? A meta-analysis. Am Nat 180, 70-82. https://doi.org/10.1086/666081

Rimbach, R., Bisanzio, D., Galvis, N., Link, A., Di Fiore, A., Gillespie, T.R., 2015. Brown spider monkeys (Ateles hybridus): A model for differentiating the role of social networks and physical contact on parasite transmission dynamics. Proc. R. Soc. B 370, 20140110. https://doi.org/10.1098/rstb.2014.0110

Rimbach, R., Heymann, E.W., Link, A., Heistermann, M., 2013. Validation of an enzyme immunoassay for assessing adrenocortical activity and evaluation of factors that affect levels of fecal glucocorticoid metabolites in two New World primates. Gen Compe Endocrinol 191, 13-23. https://doi.org/10.1016/j.ygcen.2013.05.010

Rinninella, E., Raoul, P., Cintoni, M., Franceschi, F., Miggiano, G.A.D., Gasbarrini, A., Mele, M.C., 2019. What is the healthy gut microbiota composition? A changing ecosystem across age, environment, diet, and diseases. Microorganisms 7. https://doi.org/10.3390/microorganisms7010014

Robinson, C.D., Bohannan, B.J., Britton, R.A., 2019. Scales of persistence: Transmission and the microbiome. Curr Opin Microbiol 50, 42-49. https://doi.org/10.1016/j.mib.2019.09.009

Romero, L.M., Dickens, M.J., Cyr, N.E., 2009. The reactive scope model - A new model integrating homeostasis, allostasis, and stress. Horm Behav 55, 375-389. https://doi.org/10.1016/j.yhbeh.2008.12.009

Rossanigo, C.E., Gruner, L., 1995. Moisture and temperature requirements in faeces for the development of free-living stages of gastrointestinal nematodes of sheep, cattle and deer. J Helminthol 69, 357-362. https://doi.org/10.1017/S0022149X00014954

Rothschild, D., Weissbrod, O., Barkan, E., Kurilshikov, A., Korem, T., Zeevi, D., Costea, P.I., Godneva, A., Kalka, I.N., Bar, N., Shilo, S., Lador, D., Vila, A.V., Zmora, N., Pevsner-Fischer, M., Israeli, D., Kosower, N., Malka, G., Wolf, B.C., Avnit-Sagi, T., Lotan-Pompan, M., Weinberger, A., Halpern, Z., Carmi, S., Fu, J., Wijmenga, C., Zhernakova, A., Elinav, E., Segal, E., 2018. Environment dominates over host genetics in shaping human gut microbiota. Nature 555, 210-215. https://doi.org/10.1038/nature25973

Round, J.L., Mazmanian, S.K., 2009. The gut microbiota shapes intestinal immune responses during health and disease. Nat Rev Immunol 9, 313-323. https://doi.org/10.1038/nri2515

Rudolph, K., Fichtel, C., Schneider, D., Heistermann, M., Koch, F., Daniel, R., Kappeler, P.M., 2019. One size fits all? Relationships among group size, health, and ecology indicate a lack of an optimal group size in a wild lemur population. Behav Ecol Sociobiol 73, 132. https://doi.org/10.1007/s00265-019-2746-0

Russell, Y.I., Phelps, S., 2013. How do you measure pleasure? A discussion about intrinsic costs and benefits in primate allogrooming. Biol Philos 28, 1005-1020. https://doi.org/10.1007/s10539-013-9372-4

Rutberg, A.T., 1983. Factors influencing dominance status in American bison cows (Bison bison). Z Tierpsychol 63, $206-212$. https://doi.org/10.1111/j.1439-0310.1983.tb00087.x

Saj, T.L., Marteinson, S., Chapman, C.A., Sicotte, P., 2007. Controversy over the application of current socioecological models to folivorous primates: Colobus vellerosus fits the predictions. Am J Phys Anthropol133, 994-1003.

https://doi.org/10.1002/ajpa.20609 
Sánchez-Villagra, M.R., Pope, T.R., Salas, V., 1998. Relation of intergroup variation in allogrooming to group social structure and ectoparasite loads in Red howlers (Alouatta seniculus). Int J Primatol19, 473-491. https://doi.org/10.1023/A:1020360422305

Sands, J., Creel, S., 2004. Social dominance, aggression and faecal glucocorticoid levels in a wild population of wolves, Canis lupus. Anim Behav 67, 387-396. https://doi.org/10.1016/j.anbehav.2003.03.019

Santema, P., Teitel, Z., Manser, M., Bennett, N., Clutton-Brock, T., 2013. Effects of cortisol administration on cooperative behavior in meerkat helpers. Behav Ecol 24, 1122-1127. https://doi.org/10.1093/beheco/art039

Sapolsky, R.M., 2005. The influence of social hierarchy on primate health. Science 308, 648-652. https://doi.org/10.1126/science.1106477

Sapolsky, R.M., Alberts, S.C., Altmann, J., 1997. Hypercortisolism associated with social subordinance or social isolation among wild baboons. Arch Gen Psychiatry 54, 1137-1143. https://doi.org/10.1001/archpsyc.1997.01830240097014

Sapolsky, R.M., Romero, L.M., Munck, A.U., 2000. How do glucocorticoids influence stress responses? Integrating permissive, suppressive, stimulatory, and preparative actions. Endocr Rev 21, 55-89. https://doi.org/10.1210/edrv.21.1.0389

Scheumann, M., Rabesandratana, A., Zimmermann, E., 2007. Predation, communication, and cognition in lemurs, in: Gursky, S.L., Nekaris, K.A.I. (Eds.), Primate Anti-Predator Strategies, Developments in Primatology: Progress and Prospects. Springer US, pp. 100-126.

Schielzeth, H., 2010. Simple means to improve the interpretability of regression coefficients. Methods Ecol Evol 1, 103-113. https://doi.org/10.1111/j.2041-210X.2010.00012.x

Schiml, P.A., Mendoza, S.P., Saltzman, W., Lyons, D.M., Mason, W.A., 1999. Annual physiological changes in individually housed squirrel monkeys (Saimiri sciureus). Am J Primatol 47, 93-103. https://doi.org/10.1002/(SICI)10982345(1999)47:2<93::AID-AJP1>3.0.CO;2-S

Schino, G., Perretta, G., Taglioni, A.M., Monaco, V., Troisi, A., 1996. Primate displacement activities as an ethopharmacological model of anxiety. Anxiety 2, 186-191. https://doi.org/10.1002/(SICI)1522-7154(1996)2:4<186::AID-ANXI5>3.0.CO;2M

Schino, G., Troisi, A., Perretta, G., Monaco, V., 1991. Measuring anxiety in nonhuman primates: Effect of lorazepam on macaque scratching. Pharmacol Biochem Behav 38, 889-891. https://doi.org/10.1016/0091-3057(91)90258-4

Schmid, J., Speakman, J.R., 2000. Daily energy expenditure of the grey mouse lemur (Microcebus murinus): A small primate that uses torpor. J Comp Physiol B 170, 633-641. https://doi.org/10.1007/s003600000146

Schmid-Hempel, P., 2017. Parasites and their social hosts. Trends Parasitol 33, 453-462. https://doi.org/10.1016/j.pt.2017.01.003

Schmidt, T.S.B., Hayward, M.R., Coelho, L.P., Li, S.S., Costea, P.I., Voigt, A.Y., Wirbel, J., Maistrenko, O.M., Alves, R.J.C., Bergsten, E., de Beaufort, C., Sobhani, I., Heintz-Buschart, A., Sunagawa, S., Zeller, G., Wilmes, P., Bork, P., 2019. Extensive transmission of microbes along the gastrointestinal tract. eLife 8, e42693.

Schoof, V. a. M., Jack, K.M., 2013. The association of intergroup encounters, dominance status, and fecal androgen and glucocorticoid profiles in wild male White-faced capuchins (Cebus capucinus). Am J Primatol. https://doi.org/10.1002/ajp.22089

Scotti, M.-A.L., Rendon, N.M., Greives, T.J., Romeo, R.D., Demas, G.E., 2015. Short-day aggression is independent of changes in cortisol or glucocorticoid receptors in male Siberian hamsters (Phodopus sungorus). J Exp Zool A Ecol Genet Physiol 323, 331-342. https://doi.org/10.1002/jez.1922

Semel, B.P., Baden, A.L., Salisbury, R.L., McGee, E.M., Wright, P.C., Arrigo-Nelson, S.J., 2019. Assessing the function of geophagy in a Malagasy rain forest lemur. Biotropica 51, 769-780. https://doi.org/10.1111/btp.12699

Setchell, J.M., Charpentier, M., Wickings, E.J., 2005. Mate guarding and paternity in mandrills: factors influencing alpha male monopoly. Anim Behav 70, 1105-1120. https://doi.org/10.1016/j.anbehav.2005.02.021

Shannon, C.E., 1948. A mathematical theory of communication. Bell Sys Tech. J 27, 379-423. https://doi.org/10.1002/j.15387305.1948.tb01338.x

Sheldon, B.C., Verhulst, S., 1996. Ecological immunology: Costly parasite defences and trade-offs in evolutionary ecology. Trends Ecol Evol 11, 317-321. https://doi.org/10.1016/0169-5347(96)10039-2

Shutt, K., Setchell, J.M., Heistermann, M., 2012. Non-invasive monitoring of physiological stress in the Western lowland gorilla (Gorilla gorilla gorilla): Validation of a fecal glucocorticoid assay and methods for practical application in the field. Gen Comp Endocrinol 179, 167-177. https://doi.org/10.1016/j.ygcen.2012.08.008

Silk, J.B., 2014. Evolutionary perspectives on the links between close social bonds, health, and fitness, in: Sociality, Hierarchy, Health: Comparative Biodemography: A Collection of Papers. National Academies Press, Washington D.C., pp. 121143.

Silk, J.B., 2007. Social components of fitness in primate groups. Science 317, 1347-1351. https://doi.org/10.1126/science.1140734

Silk, J.B., Alberts, S.C., Altmann, J., 2003. Social bonds of female baboons enhance infant survival. Science 302, 1231-1234. https://doi.org/10.1126/science.1088580

Silk, J.B., Beehner, J.C., Bergman, T.J., Crockford, C., Engh, A.L., Moscovice, L.R., Wittig, R.M., Seyfarth, R.M., Cheney, D.L., 2010. Strong and consistent social bonds enhance the longevity of female baboons. Curr Biol 20, 1359-1361. https://doi.org/10.1016/j.cub.2010.05.067

Silk, J.B., Kappeler, P.M., 2017. Sociality in primates, in: Comparative Social Evolution. Cambridge University Press, Cambridge.

Simmen, B., Bayart, F., Rasamimanana, H., Zahariev, A., Blanc, S., Pasquet, P., 2010. Total energy expenditure and body composition in two free-living sympatric lemurs. PLOS ONE 5, e9860. https://doi.org/10.1371/journal.pone.0009860 
Skytte, K.A., Kirkegaard, R.H., Karst, S.M., Albertsen, M., 2018. ampvis2: an R package to analyse and visualise $16 \mathrm{~S}$ rRNA amplicon data. bioRxiv.

Smith, C.C., Snowberg, L.K., Caporaso, J.G., Knight, R., Bolnick, D.I., 2015. Dietary input of microbes and host genetic variation shape among-population differences in stickleback gut microbiota. ISME J 9, 2515-2526. https://doi.org/10.1038/ismej.2015.64

Smith, R., 2008. The end of disease and the beginning of health. The BMJ. URL https://blogs.bmj.com/bmj/2008/07/08/richardsmith-the-end-of-disease-and-the-beginning-of-health/ (accessed 12.9.19).

Snaith, T.V., Chapman, C.A., 2008. Red colobus monkeys display alternative behavioral responses to the costs of scramble competition. Behav Ecol 19, 1289-1296. https://doi.org/10.1093/beheco/arn076

Snyder-Mackler, N., Sanz, J., Kohn, J.N., Brinkworth, J.F., Morrow, S., Shaver, A.O., Grenier, J.-C., Pique-Regi, R., Johnson, Z.P., Wilson, M.E., Barreiro, L.B., Tung, J., 2016. Social status alters immune regulation and response to infection in macaques. Science 354, 1041-1045. https://doi.org/10.1126/science.aah3580

Soares, M.C., Oliveira, R.F., Ros, A.F.H., Grutter, A.S., Bshary, R., 2011. Tactile stimulation lowers stress in fish. Nature Communications 2, 534. https://doi.org/10.1038/ncomms1547

Song, S.J., Lauber, C., Costello, E.K., Lozupone, C.A., Humphrey, G., Berg-Lyons, D., Caporaso, J.G., Knights, D., Clemente, J.C., Nakielny, S., Gordon, J.I., Fierer, N., Knight, R., 2013. Cohabiting family members share microbiota with one another and with their dogs. elife 2, e00458-e00458. https://doi.org/10.7554/eLife.00458

Sorg, J.P., Rohner, U., 1996. Climate and tree phenology of the dry deciduous forest of the Kirindy Forest. Primate report 46, 57-80.

Springer, A., 2015. Springer, Andrea. Patterns of parasitism in wild Verreaux's sifakas (Propithecus verreauxi) at Kirindy Forest, Madagascar: Assessing the role of host behavior. Veterinary University of Hannover, Hannover.

Springer, A., Fichtel, C., Al-Ghalith, G.A., Koch, F., Amato, K.R., Clayton, J.B., Knights, D., Kappeler, P.M., 2017. Patterns of seasonality and group membership characterize the gut microbiota in a longitudinal study of wild Verreaux's sifakas (Propithecus verreauxi). Ecol Evol https://doi.org/10.1002/ece3.3148

Springer, A., Kappeler, P.M., 2016. Intestinal parasite communities of six sympatric lemur species at Kirindy Forest, Madagascar. Primate Biol 3, 51-63. https://doi.org/10.5194/pb-3-51-2016

Springer, A., Mellmann, A., Fichtel, C., Kappeler, P.M., 2016. Social structure and Escherichia coli sharing in a group-living wild primate, Verreaux's sifaka. BMC Ecology 16, 6. https://doi.org/10.1186/s12898-016-0059-y

Srivathsan, A., Ang, A., Vogler, A.P., Meier, R., 2016. Fecal metagenomics for the simultaneous assessment of diet, parasites, and population genetics of an understudied primate. Front Zool13, 17. https://doi.org/10.1186/s12983-016-0150-4

Steenbeek, R., Piek, R.C., van Buul, M., van Hooff, J.A.R.A.M., 1999. Vigilance in wild Thomas's langurs (Presbytis thomasi): The importance of infanticide risk. Behav Ecol Sociobiol 45, 137-150. https://doi.org/10.1007/s002650050547

Steenbeek, R., van Schaik, C.P., 2001. Competition and group size in Thomas's langurs (Presbytis thomasi): The folivore paradox revisited. Behav Ecol Sociobiol 49, 100-110. https://doi.org/10.1007/s002650000286

Stevenson, P.R., Castellanos, M.C., 2000. Feeding rates and daily path range of the Colombian woolly monkeys as evidence for between- and within-group competition. Fol Primatol 71, 399-408. https://doi.org/10.1159/000052737

Stoeck, T., Bass, D., Nebel, M., Christen, R., Jones, M.D.M., Breiner, H.-W., Richards, T.A., 2010. Multiple marker parallel tag environmental DNA sequencing reveals a highly complex eukaryotic community in marine anoxic water. Mol. Ecol. 19 Suppl 1, 21-31. https://doi.org/10.1111/j.1365-294X.2009.04480.x

Stopka, P., Graciasová, R., 2001. Conditional allogrooming in the herb-field mouse. Behav Ecol 12, 584-589. https://doi.org/10.1093/beheco/12.5.584

Stothart, M.R., Palme, R., Newman, A.E.M., 2019. It's what's on the inside that counts: stress physiology and the bacterial microbiome of a wild urban mammal. Proc. R. Soc. B 286, 20192111. https://doi.org/10.1098/rspb.2019.2111

Strandburg-Peshkin, A., Farine, D.R., Crofoot, M.C., Couzin, I.D., 2017. Habitat and social factors shape individual decisions and emergent group structure during baboon collective movement. eLife 6.

Straub, R.H., Cutolo, M., Buttgereit, F., Pongratz, G., 2010. Energy regulation and neuroendocrine-immune control in chronic inflammatory diseases. J Internal Med 267, 543-560. https://doi.org/10.1111/j.1365-2796.2010.02218.x

Strier, K.B., Lynch, J.W., Ziegler, T.E., 2003. Hormonal changes during the mating and conception seasons of wild northern muriquis (Brachyteles arachnoides hypoxanthus). Am J Primatol 61, 85-99. https://doi.org/10.1002/ajp.10109

Strier, K.B., Ziegler, T.E., Wittwer, D.J., 1999. Seasonal and social correlates of fecal testosterone and cortisol levels in wild male Muriquis (Brachyteles arachnoides). Horm Behav 35, 125-134. https://doi.org/10.1006/hbeh.1998.1505

Struhsaker, T.T., 1969. Correlates of ecology and social organization among African cercopithecines. FPR 11, 80-118. https://doi.org/10.1159/000155259

Sudo, N., 2014. Microbiome, HPA axis and production of endocrine hormones in the gut, in: Lyte, M., Cryan, J.F. (Eds.), Microbial Endocrinology: The Microbiota-Gut-Brain Axis in Health and Disease, Advances in Experimental Medicine and Biology. Springer New York, pp. 177-194. https://doi.org/10.1007/978-1-4939-0897-4_8

Sussman, R.W., Garber, P.A., 2004. Rethinking sociality: Cooperation and aggression among primates. The origins and nature of sociality 161-190.

Sussman, R.W., Richard, A.F., Ratsirarson, J., Sauther, M.L., Brockman, D.K., Gould, L., Lawler, R., Cuozzo, F.P., 2012. Beza Mahafaly Special Reserve: Long-term research on lemurs in southwestern Madagascar, in: Kappeler, P.M., Watts, D.P. (Eds.), Long-Term Field Studies of Primates. Springer Berlin, Berlin, pp. 45-66. https://doi.org/10.1007/978-3-64222514-7_3 
Sutherst, R.W., Bourne, A.S., 2006. The effect of desiccation and low temperature on the viability of eggs and emerging larvae of the tick, Rhipicephalus (Boophilus) microplus (Canestrini) (Ixodidae). Int J Parasitol36, 193-200. https://doi.org/10.1016/j.ijpara.2005.09.007

Szathmáry, E., Smith, J.M., 1995. The major evolutionary transitions. Nature 374, 227-232. https://doi.org/10.1038/374227a0

Tanaka, R., Hino, A., Tsai, I.J., Palomares-Rius, J.E., Yoshida, A., Ogura, Y., Hayashi, T., Maruyama, H., Kikuchi, T., 2014. Assessment of helminth biodiversity in wild rats using 18S rDNA based metagenomics. PLOS ONE 9, e110769. https://doi.org/10.1371/journal.pone.0110769

Tarjuelo, R., Barja, I., Morales, M.B., Traba, J., Benítez-López, A., Casas, F., Arroyo, B., Delgado, M.P., Mougeot, F., 2015. Effects of human activity on physiological and behavioral responses of an endangered steppe bird. Behav Ecol 26, 828-838. https://doi.org/10.1093/beheco/arv016

Theis, F.J., Ugelvig, L.V., Marr, C., Cremer, S., 2015. Opposing effects of allogrooming on disease transmission in ant societies. Proc. R. Soc. B 370, 20140108. https://doi.org/10.1098/rstb.2014.0108

Theis, K.R., Schmidt, T.M., Holekamp, K.E., 2012. Evidence for a bacterial mechanism for group-specific social odors among hyenas. Sci Rep 2, 615. https://doi.org/10.1038/srep00615

Thierry, A.-M., Brajon, S., Spée, M., Raclot, T., 2014. Differential effects of increased corticosterone on behavior at the nest and reproductive output of chick-rearing Adélie penguins. Behav Ecol Sociobiol 68, 721-732. https://doi.org/10.1007/s00265-014-1685-z

Thomas, A.M., Segata, N., 2019. Multiple levels of the unknown in microbiome research. BMC Biology 17, 48. https://doi.org/10.1186/s12915-019-0667-z

Thouless, C.R., Guinness, F.E., 1986. Conflict between red deer hinds: the winner always wins. Anim Behav 34, 1166-1171. https://doi.org/10.1016/S0003-3472(86)80176-2

Thrall, P.H., Antonovics, J., Dobson, A.P., 2000. Sexually transmitted diseases in polygynous mating systems: prevalence and impact on reproductive success. Proc. R. Soc. B 267, 1555-1563. https://doi.org/10.1098/rspb.2000.1178

Tinbergen, N., 1951. The study of instinct. Clarendon Press/Oxford University Press, New York, NY, US.

Tkaczynski, P., MacLarnon, A., Ross, C., 2014. Associations between spatial position, stress and anxiety in forest baboons Papio anubis. Behav Proc 108, 1-6. https://doi.org/10.1016/j.beproc.2014.08.006

Touma, C., Palme, R., 2005. Measuring fecal glucocorticoid metabolites in mammals and birds: the importance of validation. Ann. N. Y. Acad. Sci. 1046, 54-74. https://doi.org/10.1196/annals.1343.006

Touma, C., Sachser, N., Möstl, E., Palme, R., 2003. Effects of sex and time of day on metabolism and excretion of corticosterone in urine and feces of mice. Gen Comp Endocrinol 130, 267-278. https://doi.org/10.1016/S0016-6480(02)00620-2

Traniello, J.F.A., Rosengaus, R.B., Savoie, K., 2002. The development of immunity in a social insect: evidence for the group facilitation of disease resistance. Proc. Natl. Acad. Sci. U.S.A. 99, 6838-6842. https://doi.org/10.1073/pnas.102176599

Trosvik, P., de Muinck, E.J., Rueness, E.K., Fashing, P.J., Beierschmitt, E.C., Callingham, K.R., Kraus, J.B., Trew, T.H., Moges, A., Mekonnen, A., Venkataraman, V.V., Nguyen, N., 2018. Multilevel social structure and diet shape the gut microbiota of the gelada monkey, the only grazing primate. Microbiome 6, 84. https://doi.org/10.1186/s40168-018-0468-6

Tung, J., Barreiro, L.B., Burns, M.B., Grenier, J.-C., Lynch, J., Grieneisen, L.E., Altmann, J., Alberts, S.C., Blekhman, R., Archie, E.A., 2015. Social networks predict gut microbiome composition in wild baboons. elife 4. https://doi.org/10.7554/eLife.05224

Turnbaugh, P.J., Hamady, M., Yatsunenko, T., Cantarel, B.L., Duncan, A., Ley, R.E., Sogin, M.L., Jones, W.J., Roe, B.A., Affourtit, J.P., Egholm, M., Henrissat, B., Heath, A.C., Knight, R., Gordon, J.I., 2009. A core gut microbiome in obese and lean twins. Nature 457, 480-484. https://doi.org/10.1038/nature07540

Turnbaugh, P.J., Ley, R.E., Mahowald, M.A., Magrini, V., Mardis, E.R., Gordon, J.I., 2006a. An obesity-associated gut microbiome with increased capacity for energy harvest. Nature 444, 1027.

Turnbaugh, P.J., Ley, R.E., Mahowald, M.A., Magrini, V., Mardis, E.R., Gordon, J.I., 2006b. An obesity-associated gut microbiome with increased capacity for energy harvest. Nature 444, 1027-1031. https://doi.org/10.1038/nature05414

Turnbull, C., Hoggard, S., Gillings, M., Palmer, C., Stow, A., Beattie, D., Briscoe, D., Smith, S., Wilson, P., Beattie, A., 2011. Antimicrobial strength increases with group size: Implications for social evolution. Biol Lett 7, 249-252. https://doi.org/10.1098/rsbl.2010.0719

van de Belt, T.H., Engelen, L.J., Berben, S.A., Schoonhoven, L., 2010. Definition of health 2.0 and medicine 2.0: A systematic review. J Med Internet Res 12, e18. https://doi.org/10.2196/jmir.1350

van Meter, P.E., French, J.A., Dloniak, S.M., Watts, H.E., Kolowski, J.M., Holekamp, K.E., 2009. Fecal glucocorticoids reflect socio-ecological and anthropogenic stressors in the lives of wild spotted hyenas. Horm Behav 55, 329-337. https://doi.org/10.1016/j.yhbeh.2008.11.001

van Schaik, C.P., Kappeler, P.M., 1996. The social systems of gregarious lemurs: Lack of convergence with anthropoids due to evolutionary disequilibrium? Ethology 102, 915-941. https://doi.org/10.1111/j.1439-0310.1996.tb01171.x

van Schaik, C.P., Noordwijk, M.A. van, Boer, R.J. de, Tonkelaar, I. den, 1983. The effect of group size on time budgets and social behaviour in wild long-tailed macaques (Macaca fascicularis). Behav Ecol Sociobiol 13, 173-181. https://doi.org/10.1007/BF00299920

Veldhuis, J.D., Sharma, A., Roelfsema, F., 2013. Age-dependent and gender-dependent regulation of HypothalamicAdrenocorticotropic-Adrenal axis. Endocrinol Metabol Clinics 42, 201-225. https://doi.org/10.1016/j.ecl.2013.02.002

Vera, F., Zenuto, R.R., Antenucci, C.D., 2013. Seasonal variations in plasma cortisol, testosterone, progesterone and leukocyte profiles in a wild population of tuco-tucos. J Zool 289, 111-118. https://doi.org/10.1111/j.1469-7998.2012.00967.x

Verbrugge, L.M., 1979. Marital status and health. J Marriage Fam 41, 267-285. https://doi.org/10.2307/351696 
Vlčková, K., Shutt-Phillips, K., Heistermann, M., Pafčo, B., Petrželková, K.J., Todd, A., Modrý, D., Nelson, K.E., Wilson, B.A., Stumpf, R.M., White, B.A., Leigh, S.R., Gomez, A., 2018. Impact of stress on the gut microbiome of free-ranging western lowland gorillas. Microbiology, 164, 40-44. https://doi.org/10.1099/mic.0.000587

Voellmy, I.K., Goncalves, I.B., Barrette, M.-F., Monfort, S.L., Manser, M.B., 2014. Mean fecal glucocorticoid metabolites are associated with vigilance, whereas immediate cortisol levels better reflect acute anti-predator responses in meerkats. Horm Behav66, 759-765. https://doi.org/10.1016/j.yhbeh.2014.08.008

Walburn, J., Vedhara, K., Hankins, M., Rixon, L., Weinman, J., 2009. Psychological stress and wound healing in humans: a systematic review and meta-analysis. J Psychosom Res 67, 253-271. https://doi.org/10.1016/j.jpsychores.2009.04.002

Ward, A., Webster, M., 2016. The evolution of group living, in: Sociality: The Behaviour of Group-Living Animals. Springer, Cham, pp. 191-216. https://doi.org/10.1007/978-3-319-28585-6_10

Wasimuddin, Menke, S., Melzheimer, J., Thalwitzer, S., Heinrich, S., Wachter, B., Sommer, S., 2017. Gut microbiomes of freeranging and captive Namibian cheetahs: Diversity, putative functions and occurrence of potential pathogens. Mol Ecol 26, 5515-5527. https://doi.org/10.1111/mec.14278

Wasser, S.K., Thomas, R., Nair, P.P., Guidry, C., Southers, J., Lucas, J., Wildt, D.E., Monfort, S.L., 1993. Effects of dietary fibre on faecal steroid measurements in baboons (Papio cynocephalus cynocephalus). Reproduction 97, 569-574. https://doi.org/10.1530/jrf.0.0970569

Webster, J.P., Borlase, A., Rudge, J.W., 2017. Who acquires infection from whom and how? Disentangling multi-host and multimode transmission dynamics in the 'elimination' era. Proc. R. Soc. B 372, 20160091. https://doi.org/10.1098/rstb.2016.0091

Welbergen, J.A., 2011. Fit females and fat polygynous males: seasonal body mass changes in the grey-headed flying fox. Oecologia 165, 629-637. https://doi.org/10.1007/s00442-010-1856-1

Wey, T., Blumstein, D.T., Shen, W., Jordán, F., 2008. Social network analysis of animal behaviour: a promising tool for the study of sociality. Anim Behav 75, 333-344. https://doi.org/10.1016/j.anbehav.2007.06.020

Wheeler, B.C., Scarry, C.J., Koenig, A., 2013. Rates of agonism among female primates: a cross-taxon perspective. Behav Ecol 24, 1369-1380. https://doi.org/10.1093/beheco/art076

White, L.A., Forester, J.D., Craft, M.E., 2017. Using contact networks to explore mechanisms of parasite transmission in wild life. Biol Rev 92, 389-409. https://doi.org/10.1111/brv.12236

Whitehouse, J., Micheletta, J., Kaminski, J., Waller, B.M., 2016. Macaques attend to scratching in others. Anil Behav 122, 169175. https://doi.org/10.1016/j.anbehav.2016.10.020

Widom, C.S., 1999. Posttraumatic stress disorder in abused and neglected children grown up. AJP 156, 1223-1229. https://doi.org/10.1176/ajp.156.8.1223

Wikberg, E., Christie, D., Sicotte, P., Ting, N., 2019. Social interactions across groups of colobus monkeys (Colobus vellerosus) explain similarities in their gut microbiomes. bioRxiv 717934. https://doi.org/10.1101/717934

Williams, C.L., Willard, S., Kouba, A., Sparks, D., Holmes, W., Falcone, J., Williams, C.H., Brown, A., 2013. Dietary shifts affect the gastrointestinal microflora of the giant panda (Ailuropoda melanoleuca). J Anim Physiol Anim Nutr 97, 577-585. https://doi.org/10.1111/j.1439-0396.2012.01299.x

Wilson, E.O., 1975. Sociobiology: The new synthesis. Belknap Press of Harvard U Press, Oxford, England.

Wilson, J.J., Rougerie, R., Schonfeld, J., Janzen, D.H., Hallwachs, W., Hajibabaei, M., Kitching, I.J., Haxaire, J., Hebert, P.D., 2011. When species matches are unavailable are DNA barcodes correctly assigned to higher taxa? An assessment using sphingid moths. BMC Ecology 11, 18. https://doi.org/10.1186/1472-6785-11-18

Wimmer, B., Craig, B.H., Pilkington, J.G., Pemberton, J.M., 2004. Non-invasive assessment of parasitic nematode species diversity in wild Soay sheep using molecular markers. Int J Parasitol 34, 625-631. https://doi.org/10.1016/j.ijpara.2003.11.022

Wittig, R.M., Crockford, C., Lehmann, J., Whitten, P.L., Seyfarth, R.M., Cheney, D.L., 2008. Focused grooming networks and stress alleviation in wild female baboons. Horm Behav54, 170-177. https://doi.org/10.1016/j.yhbeh.2008.02.009

Wittig, R.M., Crockford, C., Weltring, A., Langergraber, K.E., Deschner, T., Zuberbühler, K., 2016. Social support reduces stress hormone levels in wild chimpanzees across stressful events and everyday affiliations. Nat Commun 7, 13361. https://doi.org/10.1038/ncomms13361

World Health Organization, 1948. Preamble to the constitution of the World Health Organization as adopted by the International Health Conference, New York, 19-22 June, 1946 ; http://www.who.int/governance/eb/who_constitution_en.pdf.

Wrangham, R.W., Gittleman, J.L., Chapman, C.A., 1993. Constraints on group size in primates and carnivores: population density and day-range as assays of exploitation competition. Behav Ecol Sociobiol 32, 199-209. https://doi.org/10.1007/BF00173778

Wright, P.C., 1999. Lemur traits and Madagascar ecology: Coping with an island environment. Am J Phys Anthropol110, 31-72. https://doi.org/10.1002/(SICI)1096-8644(1999)110:29+<31::AID-AJPA3>3.0.CO;2-0

Wright, P.C., 1998. Impact of predation risk on the behaviour of Propithecus diadema edwardsi in the rain forest of Madagascar. Behaviour 135, 483-512.

Wright, P.C., 1995. Demography and life history of free-ranging Propithecus diadema edwardsi in Ranomafana National Park, Madagascar. Int J Primatol 16, 835. https://doi.org/10.1007/BF02735722

Wright, P.C., Heckscher, K., Dunham, A.E., 1997. Predation on Milne-Edward's Sifaka (Propithecus diadema edwardsi) by the Fossa (Cryptoprocta ferox) in the rain forest of Southeastern Madagascar. FPR 68, 34-43. https://doi.org/10.1159/000157230 
Wu, G.D., Chen, J., Hoffmann, C., Bittinger, K., Chen, Y.-Y., Keilbaugh, S.A., Bewtra, M., Knights, D., Walters, W.A., Knight, R., Sinha, R., Gilroy, E., Gupta, K., Baldassano, R., Nessel, L., Li, H., Bushman, F.D., Lewis, J.D., 2011. Linking long-term dietary patterns with gut microbial enterotypes. Science 334, 105-108. https://doi.org/10.1126/science.1208344

Young, C., Majolo, B., Heistermann, M., Schülke, O., Ostner, J., 2014. Responses to social and environmental stress are attenuated by strong male bonds in wild macaques. PNAS 111, 18195-18200. https://doi.org/10.1073/pnas.1411450111

Youngblut, N.D., Reischer, G.H., Walters, W., Schuster, N., Walzer, C., Stalder, G., Ley, R.E., Farnleitner, A.H., 2019. Host diet and evolutionary history explain different aspects of gut microbiome diversity among vertebrate clades. Nat Commun 10, 2200. https://doi.org/10.1038/s41467-019-10191-3

Yuan, M.L., Dean, S.H., Longo, A.V., Rothermel, B.B., Tuberville, T.D., Zamudio, K.R., 2015. Kinship, inbreeding and fine-scale spatial structure influence gut microbiota in a hindgut-fermenting tortoise. Molec Ecol 24, 2521-2536. https://doi.org/10.1111/mec.13169

Zaiss, M.M., Harris, N.L., 2016. Interactions between the intestinal microbiome and helminth parasites. Parasite Immunol 38, 5-11. https://doi.org/10.1111/pim.12274

Zhang, J., Kobert, K., Flouri, T., Stamatakis, A., 2014. PEAR: a fast and accurate Illumina Paired-End reAd mergeR. Bioinformatics 30, 614-620. https://doi.org/10.1093/bioinformatics/btt593

Zoetendal, E.G., Akkermans, A.D.L., Akkermans-van Vliet, W.M., de Visser, A.G.M., de Vos, W.M., 2001. The host genotype affects the bacterial community in the human gastronintestinal tract. Microb Ecol Health Disease 13, 129-134. https://doi.org/10.1080/089106001750462669 


\section{Acknowledgements}

As the successful completion of such a dissertation is also depending on the cooperation of others. I would like to thank those who enabled me to accomplish this work.

First, I would like to express my sincere gratitude to Dr. Claudia Fichtel and Prof. Peter M. Kappeler for their academic supervision, their constructive feedback and for giving me the incredible opportunity to work with wild lemurs in Madagascar!

I am grateful to Dr. Michael Heistermann for welcoming me into his Endocrinology Lab and for his patience and support during hormone analyses. Special thanks also to Andrea Heistermann and Miriam Bolte for being there whenever I had questions and for their entertaining stories during long hours of diluting samples. It was truly a pleasure to work with all of you!

Thank you very much Dr. Dominik Schneider for introducing me to your weird world of bacteria, dirt and "fairy water". You were always patient and understanding of my microbial unknowingness and eventually even managed to get some microbial wisdom into me!

I am thankful to Prof. Julia Ostner and Prof. Rolf Daniel for being part of my thesis committee and for bringing new perspectives and ideas to my project. I also want to thank Dr. Dietmar Zinner and Prof. Lars Penke for agreeing to be part of my examination board.

Without the help of Tianasoa Andrianjanahary and Mamy Solohery Razafindrasamba and the other assistants at the field station in Kirindy Forest, this work would not have been possible. Thank you very much!

I am grateful to all past and present members of the Behavioral Ecology and Sociobiology Unit of the German Primate Center and to all the members of the Research Group "Sociality and Health in Primates" for numerous fruitful discussions during retreats, workshops and coffee breaks. A huge thank you goes to Johanna Henke - von der Malsburg for the many fun hours inside and outside of our office and for your very helpful comments and suggestions on this thesis.

Many final thanks go to my family and friends for their unfailing confidence and support. 


\section{Declaration}

I hereby declare that all parts of my thesis titled "Health consequences of group living in wild Verreaux's sifakas (Propithecus verreauxi)" were written by myself. Assistance of third parties was only accepted if scientifically justifiable and acceptable in regard to the examination regulations. Assistance or contributions to the individual chapters are indicated and all sources have been quoted.

Göttingen, $19^{\text {th }}$ of December 2019

Katja Rudolph 